JOED HENRIQUE PAES

\begin{abstract}
A COMPARATIVE STUDY OF ROBUST CONTROL METHODS APPLIED TO VEHICLE ANTI-ROLL SYSTEM BY ACTIVE SWAY BAR
\end{abstract}



JOED HENRIQUE PAES

\section{A COMPARATIVE STUDY OF ROBUST CONTROL METHODS APPLIED TO VEHICLE ANTI-ROLL SYSTEM BY ACTIVE SWAY BAR}

Dissertation submitted to Escola

Politécnica of the Universidade de São

Paulo for degree of Master in Science. 



\section{JOED HENRIQUE PAES}

\section{A COMPARATIVE STUDY OF ROBUST CONTROL METHODS APPLIED TO VEHICLE ANTI-ROLL SYSTEM BY ACTIVE SWAY BAR}

Dissertation submitted to Escola

Politécnica of the Universidade de São

Paulo for degree of Master in Science.

Concentration area:

Systems engineering

Advisor:

Prof. Dr. Diego Colón 
Autorizo a reprodução e divulgação total ou parcial deste trabalho, por qualquer meio convencional ou eletrônico, para fins de estudo e pesquisa, desde que citada a fonte.

Este exemplar foi revisado e corrigido em relação à versão original, sob responsabilidade única do autor e com a anuência de seu orientador.

São Paulo, de de

Assinatura do autor:

Assinatura do orientador:

\section{Catalogação-na-publicação}

Paes, Joed Henrique

A comparative study of robust control methods applied to vehicle anti-roll system by active sway bar / J. H. Paes -- versão corr. -- São Paulo, 2019.

$159 \mathrm{p}$.

Dissertação (Mestrado) - Escola Politécnica da Universidade de São Paulo. Departamento de Engenharia de Telecomunicações e Controle.

1.Anti-roll system 2.Flexible structures 3.Control 4.Finite Element Method I.Universidade de São Paulo. Escola Politécnica. Departamento de Engenharia de Telecomunicações e Controle II.t. 
Dedication

To God, owner of whole science... 



\section{ACKNOWLEDGMENTS}

First of all, I thank God for the opportunity to acquire new and precious knowledge through this work. I thank my wife Joice Nogueira Neris Paes for her patience and support during this period of study. I thank my parents Odair Silvério Paes and Aparecida de Fátima Spósito Paes for the trust, the love, the affection and great encouragement given to me. I thank the company Schaeffler Brasil Ltda. for the encouragement to improve my knowledge. I thank the University of São Paulo for the opportunity to expand my knowledge and to introduce me to the fantastic world of control. Special thanks to Prof. Dr. Diego Colón for his confidence, guidance, support and patience in the face of all the problems that have arisen during this work. It was very worthwhile for all the knowledge acquired. 

"And let not your behavior be like that of this world,

but be changed and made new in mind, so that by experience you may have knowledge of the good, and pleasing and complete purpose of God."

-Holy Bible, Romans 12,2- 



\section{RESUMO}

As barras estabilizadoras têm como objetivo aumentar a segurança dos veículos durante trajetórias curvelíneas. Através da redução do ângulo de rolagem, as barras minimizam o risco de capotamento durante as curvas em função da aceleração lateral, e também, como consequência, melhoram o conforto de dirigibilidade. Este trabalho apresenta estudos da dinâmica lateral do veículo em três abordagens diferentes: um veículo sem sistema de anti-rolagem, um veículo com barra estabilizadora passiva e um veículo com barra estabilizadora ativa. A barra de anti-rolagem ativa é controlada por um motor gerador de torque e uma comparação entre dois controladores robustos é apresentada. As barras são modeladas pelo FEM (Método dos Elementos Finitos) e os controladores LQG/LTR (Gaussiano Quadrático Linear/Malha de Recuperação) e $H_{\infty}$ Loop-Shaping são projetados para o sistema ativo com o objetivo de avaliar a opção mais promissora. Além disso, à dinâmica lateral do veículo é adicionado o modelo flexível da barra de antirolagem para se obter um sistema único (modelo híbrido). O trabalho revisa a dinâmica lateral do veículo e descreve de forma detalhada o FEM aplicado à modelagem dinâmica de estruturas flexíveis. A influência do sistema ativo de anti-rolagem e dos diferentes controladores é ilustrada através de simulação comparando-a com o sistema passivo e o modelo dinâmico sem sistema de anti-rolagem.

Palavras-Chave - Sistemas de anti-rolagem, estruturas flexíveis, controle, método dos elementos finitos. 



\section{ABSTRACT}

Anti-roll bars aim to increase the vehicle's safety during curved trajectories. By reducing the roll angle, the bars minimize the risk of overturning during cornering in function of the increasing lateral acceleration, and also, as a consequence, improves the steering comfort. This work presents studies of vehicle's lateral dynamics in three different approaches: a vehicle without anti-roll system, a vehicle with passive roll bar and a vehicle with an active anti-roll bar. The active anti-roll bar is controlled by a torque-generating motor and comparisons between two robust controllers is presented. The bars are modeled by FEM (Finite Element Method) and controllers LQG/LTR (Linear Quadratic Gaussian/Loop Transfer Recovery) and $H_{\infty}$ Loop-Shaping are designed to the active system in order to evaluated the best option. In addition, the vehicle's lateral dynamic is hybridized to the flexible bar model in order to obtain an unique system. It reviews the vehicle's roll dynamic and describes in detail the FEM applied to dynamic modeling of flexible structures. The influence of the active anti-roll system and the different controllers are illustrated through numerical simulation and a comparison between the passive system and the system without bar is performed.

Keywords - anti-roll system, flexible structures, control, finite element method. 



\section{LIST OF FIGURES}

$1 \quad$ Cornering forces acting on the vehicle under curved trajectory. . . . . . . . 28

2 Parameters for vehicle's static stability factor. . . . . . . . . . . . . . . 31

$3 \quad$ Passive anti-roll bar placed in a vehicle's suspension system. . . . . . . . . 33

4 Passive anti-roll bar schematics. . . . . . . . . . . . . . . . . . 33

5 Active anti-roll bar. . . . . . . . . . . . . . . . . . . . . 34

$6 \quad$ Active anti-roll bar schematics. . . . . . . . . . . . . . . . . . 34

$7 \quad$ Schematic model for the roll dynamics with passive anti-roll bar. . . . . . . 49

$8 \quad$ Roll dynamics and free body diagram. . . . . . . . . . . . . . . . . 50

$9 \quad$ Suspension forces. . . . . . . . . . . . . . . . . . . . . . . . 51

$10 \quad$ Four d.o.f. roll dynamics model. . . . . . . . . . . . . . . . . . . 53

11 Roll dynamics model with passive anti-roll bar. . . . . . . . . . . . . . 57

12 3D beam element. . . . . . . . . . . . . . . . . . . . . . . . . . 68

13 The d.o.f. for axial displacements. . . . . . . . . . . . . . . . . . . . . 69

$14 \quad$ Axial load diagram. . . . . . . . . . . . . . . . . . . . . . . . 69

15 Interpolation of axial displacement. . . . . . . . . . . . . . . . . 71

16 The d.o.f. for bending displacements in xy-plane. . . . . . . . . . . . . . 72

17 Bending load diagram. . . . . . . . . . . . . . . . . . . 73

18 The d.o.f. for bending displacements in xz-plane. . . . . . . . . . . . . . 76

19 The d.o.f. for torsion. . . . . . . . . . . . . . . . . . . . . . 77

20 Cartesian coordinate systems and table of direction cosines. . . . . . . . . 80

21 Coordinates and direction cosines, two-dimensional case. . . . . . . . . . 80

22 2D Frame element $\ldots \ldots \ldots \ldots$. . . . . . . . . . . . . . . . . 81

23 Arbitrary oriented 3D frame element. . . . . . . . . . . . . . . . . 82 
24 Geometry of the passive anti-roll bar. . . . . . . . . . . . . . 85

25 Roll dynamics model with active anti-roll bar. . . . . . . . . . . . . . . . . 88

26 Torque direction adopted for the electric motor. . . . . . . . . . . . . . . . 89

$27 \quad$ Geometry of the active anti-roll bar. $\ldots \ldots \ldots$. . . . . . . . . . . . 99

28 Bar cross-section. . . . . . . . . . . . . . . . . . . . . . . . . 101

29 Response comparison of the author's model and ADAMS/Car $₫$ : (a) steering input, (b) side-slip angle, (c) yaw rate, and (d) lateral acceleration. . . 102

$30 \quad$ Additive perturbation. . . . . . . . . . . . . . . . . . 106

31 Multiplicative perturbation. . . . . . . . . . . . . . . . 106

32 Left coprime factor perturbations. . . . . . . . . . . . . . . 106

33 General block diagram of a feedback system. . . . . . . . . . . . . . . 107

34 Barriers over the frequency range. . . . . . . . . . . . . . . . . . . 110

$35 \quad$ Barriers under influence of $e_{M}(\omega) . \ldots \ldots 112$

36 LQG scheme blocks. . . . . . . . . . . . . . . . . . . . . . . . . . . . . 113

37 The Kalman filter. . . . . . . . . . . . . . . . . . . . . . . . . . . . . . . 115

38 Structure of the LQG compensator. . . . . . . . . . . . . . . . . 116

$39 \quad$ System approach to $\rho \rightarrow 0$. . . . . . . . . . . . . . . . 118

$40 \quad$ Coprime factor robust stabilization problem. . . . . . . . . . . . . . . . 120

41 The loop-shaping design procedure. . . . . . . . . . . . . . . . . . . . . . . 122

42 Hankel singular values vs. their corresponding state. . . . . . . . . . . . . 126

43 Hankel singular values vs. order of the reduced model (first 30 states). . . 127

44 Magnitude plot of the original and reduced models for active anti-roll bar. 128

45 Error function $e_{M}(\omega)$ by multiplicative definition of uncertainty. . . . . . . 128

$46 \quad$ Loop Transfer Recovery (LTR). . . . . . . . . . . . . . . . . . . . . . . . 130

$47 \quad$ Open-loop response and the performance barriers. . . . . . . . . . . . . . . 131

$49 \quad$ Sensitivity and complementary sensitivity function for LQG/LTR. . . . . . 131 
48 Poles (x) and zeros (o) of the closed-loop system with nominal plant for LQG/LTR. . . . . . . . . . . . . . . . . . . . 132

$50 \quad$ Robust stabilization condition for LQG/LTR. . . . . . . . . . . . . . . . 132

51 Nominal plant, shaped plant and barriers for HLS. . . . . . . . . . . . . . . 133

52 Open-loop response for HLS. . . . . . . . . . . . . . . . . . 134

53 Poles $(\times)$ and zeros (o) of the closed-loop system with nominal plant for HLS. . . . . . . . . . . . . . . . . . . . . . . . 134

54 Sensitivity and complementary sensitivity function for HLS. . . . . . . . . 135

55 Robust stability condition for HLS. . . . . . . . . . . . . . . . . . . . 135

56 Block diagram for the uncontrolled system. . . . . . . . . . . . . . . . 136

57 Block diagram for the controlled system. . . . . . . . . . . . . . . . . 136

$58 \quad$ Step command in handwheel and lateral acceleration in $60 \mathrm{~km} / \mathrm{h}$ step steer test. . . . . . . . . . . . . . . . . . . . . . 137

59 Nodes d.o.f. of the passive anti-roll bar. . . . . . . . . . . . . . . 137

60 Passive anti-roll bar: bending in y-direction in $60 \mathrm{~km} / \mathrm{h}$ step steer test. . . 138

$61 \quad$ Passive anti-roll bar: torsion in $60 \mathrm{~km} / \mathrm{h}$ step steer test. . . . . . . . . . . . 139

62 Node's d.o.f.'s of the active anti-roll bar. . . . . . . . . . . . . . . . . . . . 139

63 Active anti-roll bar: bending in y-direction for the LQG/LTR and HLS in $60 \mathrm{~km} / \mathrm{h}$ step steer test. . . . . . . . . . . . . . . . . . . . 140

64 Active anti-roll bar: torsion for the LQG/LTR and HLS in $60 \mathrm{~km} / \mathrm{h}$ step steer test. . . . . . . . . . . . . . . . . . . . . . . . . . 140

65 Roll angle of the model without bar, with passive bar and active bar in 60 $\mathrm{km} / \mathrm{h}$ step steer test. . . . . . . . . . . . . . . . . . . . . . 141

66 Roll rate of the model without bar, with passive bar and active bar in 60 $\mathrm{km} / \mathrm{h}$ step steer test. . . . . . . . . . . . . . . . . . . . . . . 142

67 Control signal for both controllers: torque provided by the electric motor in $60 \mathrm{~km} / \mathrm{h}$ step steer test. . . . . . . . . . . . . . . . . . . . . . 143

68 Sinusoidal input to evaluate the disturbance rejection. . . . . . . . . . . . . 144

69 Roll angle response to the sinusoidal input at different frequencies. . . . . . 145 
70 Roll rate for the sinusoidal input at different frequencies. . . . . . . . . . 146

71 Lane-change maneuver at $90 \mathrm{~km} / \mathrm{h}:$ (a) handwheel and (b) lateral acceleration. . . . . . . . . . . . . . . . . . . . . 148

72 Passive anti-roll bar: bending in $y$ direction in $90 \mathrm{~km} / \mathrm{h}$ lane-change maneuver. . . . . . . . . . . . . . . . . . . . . . . . . . 148

$73 \quad$ Passive anti-roll bar: torsion in $90 \mathrm{~km} / \mathrm{h}$ lane-change maneuver. . . . . . . 149

74 Active anti-roll bar: bending in $y$ direction for the LQG/LTR and HLS in $90 \mathrm{~km} / \mathrm{h}$ lane-change maneuver. . . . . . . . . . . . . . . . . . . . . . . . 149

75 Torsion of the active anti-roll bar in $90 \mathrm{~km} / \mathrm{h}$ lane-change maneuver. . . . . 150

$76 \quad$ Roll angle of the model without bar, with passive bar and active bar in 90 $\mathrm{km} / \mathrm{h}$ lane-change maneuver. . . . . . . . . . . . . . . . . . . . . . 151

77 Roll rate of the model without bar, with passive bar and active bar in 90 $\mathrm{km} / \mathrm{h}$ lane-change maneuver. . . . . . . . . . . . . . . . . . . . . 151

78 Control signal for both controllers: torque provided by the electric motor in $90 \mathrm{~km} / \mathrm{h}$ lane-change maneuver. . . . . . . . . . . . . . . . . . . 153

79 Angular velocity of the electric motor for both controllers in $90 \mathrm{~km} / \mathrm{h}$ lanechange maneuver. . . . . . . . . . . . . . . . . . . . . . 153

80 Instantaneous power of the electric motor for both controllers in $90 \mathrm{~km} / \mathrm{h}$ lane-change maneuver. . . . . . . . . . . . . . . . . . . . . . 154 


\section{LIST OF TABLES}

$1 \quad$ Comparison of solutions to Equation $2.35 \mid$ at $x=0.5 \ldots \ldots$. . . . . . 47

$2 \quad$ Node's spatial coordinates, $\mathrm{x}-\mathrm{y}-\mathrm{z}$.

$3 \quad$ Designation of the parameters. . . . . . . . . . . . . . . . . . . 99

4 Geometric coordinates of active bar A. . . . . . . . . . . . . . . . 100

5 Geometric coordinates of active bar B. . . . . . . . . . . . . . . . 100

$6 \quad$ Model's parameters values. . . . . . . . . . . . . . . . . . . . 101

$7 \quad$ Percentage of improvement in $60 \mathrm{~km} / \mathrm{h}$ step steer test. . . . . . . . . . . . 142

$8 \quad$ Percentage of improvement in $40 \mathrm{~km} / \mathrm{h}$ sinusoidal steer test at $0.2 \mathrm{~Hz}$. . . . 147

$9 \quad$ Percentage of improvement in $40 \mathrm{~km} / \mathrm{h}$ sinusoidal steer test at $0.7 \mathrm{~Hz}$. . . . 147

10 Percentage of improvement in $40 \mathrm{~km} / \mathrm{h}$ sinusoidal steer test at $2 \mathrm{~Hz}$. . . . . 147

11 Percentage of improvement in $90 \mathrm{~km} / \mathrm{h}$ lane-change maneuver. . . . . . . . 152 



\section{LIST OF SYMBOLS}

$l$ lateral distance between left and right suspensions

$l_{f, r}$ distance from c.g. to the front/rear wheels

$h$ height of vehicle's c.g. from roll center

$a_{y}$ vehicle's lateral acceleration

$m$ vehicle's total mass

$m_{s}$ vehicle's sprung mass

$m_{u}$ vehicle's unsprung mass on either left or right side

$I_{z}$ yaw moment of inertia about c.g.

$I_{x x}$ roll moment of inertia about c.g.

$I_{w}$ moment of inertia of the two front wheels

$C_{f, r}$ front/rear tire lateral stiffness

$g$ acceleration due to gravity

$F_{l a t}$ total lateral tire force (from front and rear tires)

$F_{t l}$ left tire dynamic force

$F_{t r}$ right tire dynamic force

$V_{x}$ vehicle's longitudinal velocity

$N_{G}$ steering ratio

$\phi$ roll angle

$\psi$ yaw angle

$\beta$ side-slip angle

$\alpha$ handwheel angle

$z_{s}$ vehicle's vertical deflection of c.g.

$z_{u l}$ vertical deflection of left unsprung mass

$z_{u r}$ vertical deflection of right unsprung mass

$z_{r l}$ left road displacement input

$z_{r r}$ right road displacement input 
$K_{s}$ steering spring stiffness

$k_{s}$ suspension stiffness

$C_{s}$ steering viscous damping ratio

$b_{s}$ suspension damping coefficient

$k_{t}$ tire stiffness

$\epsilon$ mechanical trail

$L$ Lagrangian

$v$ nodal displacements

$E I$ bending rigidity

$\rho$ mass density per unit length

$\nu$ Poisson's ratio

$k_{v}$ element stiffness matrix

$d_{v}$ element damping matrix

$m_{v}$ element mass matrix

$K_{v}$ global stiffness matrix

$D_{v}$ global damping matrix

$M_{v}$ global mass matrix

$P_{\text {rot }}$ rotational power

$T$ torque

$\omega$ angular velocity

$E_{u}$ control energy

$X_{R M S}$ RMS value of $\mathrm{X}$

() time derivative

()$^{n}$ n-order spacial derivative 


\section{LIST OF ACRONYMS}

c.g. center of gravity

d.o.f. degree of freedom

SSF static stability factor

SUV sport utility vehicle

MPC model predictive controllers

FEM finite element method

AARB active anti-roll bar

PARB passive anti-roll bar

ESP electronic stability program

LSC longitudinal speed control

LQ linear quadratic

SOF static output feedback

ARE algebraic Riccati equation

SISO single-input-single-output

MIMO multiple-input-multiple-output

HLS $H_{\infty}$ loop-shaping

LQG linear quadratic Gaussian

LTR loop transfer recovery

RMS root mean square 



\section{CONTENTS}

\begin{tabular}{lll}
\hline 1 & Introduction & 27 \\
\hline
\end{tabular}

1.1 Published work . . . . . . . . . . . . . . . . . . 27

1.2 Motivation . . . . . . . . . . . . . . . . . . . 27

1.3 Literature survey $\ldots \ldots \ldots \ldots$

1.4 Anti-roll systems . . . . . . . . . . . . . . . . . . . . . . 31

1.4 .1 Anti-roll bars . . . . . . . . . . . . . . . . . . . . 31

1.4 .2 Passive anti-roll bars . . . . . . . . . . . . . . . . . . . . 32

1.4 .3 Active anti-roll bars . . . . . . . . . . . . . . . . . . 33

1.5 Structure of the thesis . . . . . . . . . . . . . . . . . 35

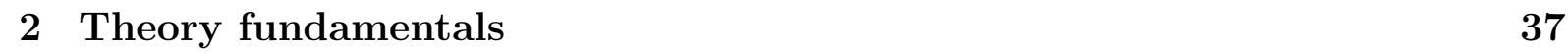

2.1 Analytical mechanics $\ldots \ldots \ldots \ldots$. . . . . . . . . . . . . . 37

2.1 .1 The principle of virtual work . . . . . . . . . . . . . . . . 37

$2.1 .2 \quad$ D'Alembert's principle . . . . . . . . . . . . . . . . . . . 38

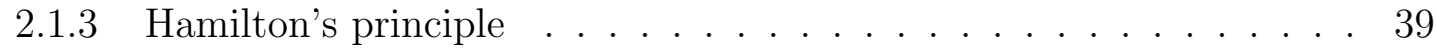

2.2 Dynamic of flexible structures f . . . . . . . . . . . . . . . . . 40

$2.2 .1 \quad$ Flexible structures $\ldots \ldots \ldots$. . . . . . . . . . . . . . . . . . 40

2.2 .2 State-space representations . . . . . . . . . . . . . . . . . . 40

$2.2 .3 \quad$ Balanced open-loop systems . . . . . . . . . . . . . . . . . . . . 41

2.2.3.1 Observability and controllability grammians . . . . . . . . 42

$2.2 .4 \quad$ Balanced representation $\ldots \ldots \ldots \ldots$

2.2 .5 Model reduction . . . . . . . . . . . . . . . . . . . . . . . . . . . . 44

2.3 Finite element method . . . . . . . . . . . . . . . . . . . . . . . . . . . 45

2.3 .1 Method of weighted residuals . . . . . . . . . . . . . . . 45 
3 Mathematical model: basic dynamic equations of the passive system 49

3.1 Four d.o.f. roll dynamics model . . . . . . . . . . . . . . . . . . . . . . 52

$3.1 .1 \quad$ State-space model . . . . . . . . . . . . . . . . . . . . 55

3.2 Inclusion of the passive anti-roll bar . . . . . . . . . . . . . . . 56

3.2 .1 Non-conservative work $W_{n c} \ldots \ldots \ldots \ldots$

$3.2 .2 \quad$ Equations of motion $\ldots \ldots \ldots \ldots$. . . . . . . . . . . . . 59

3.3 Search for solutions . . . . . . . . . . . . . . . . . . . . . . . . . 63

$3.4 \quad$ Hybridized model: FEM and discrete equations . . . . . . . . . . . . . . 64

$\begin{array}{llll}4 & \text { Passive Anti-roll bar FEM Model } & 67\end{array}$

4.1 Beam element in three dimensions . . . . . . . . . . . . . . . . . . . . 67

$4.1 .1 \quad$ Axial deformation, $v_{x}^{(i)} \ldots \ldots \ldots \ldots$

Strong formulation. . . . . . . . . . . . . . . . 69

Weak formulation. . . . . . . . . . . . . . . . 70

Domain discretization. . . . . . . . . . . . . 71

4.1.2 Bending deformation in the xy-plane, $v_{y}^{(i)} \ldots \ldots \ldots$. . . . . . 72

Strong formulation. . . . . . . . . . . . . . . . . 72

Weak formulation. . . . . . . . . . . . . . . . 74

Domain discretization. . . . . . . . . . . . . . . 75

$4.1 .3 \quad$ Bending deformation in the xz-plane, $v_{z}^{(i)} \ldots \ldots \ldots$. . . . . 76

4.1 .4 Torsional deformation, $\theta_{x}^{(i)} \ldots \ldots \ldots \ldots \ldots$

4.2 Coordinate transformation $\ldots \ldots \ldots \ldots$

4.2 .1 Transformation of element stiffness in two dimensions . . . . . . . . 80

4.2 .2 Transformation of element stiffness in three dimensions . . . . . . . 82

4.3 Assembly of elements . . . . . . . . . . . . . . . . . . . . 83

4.4 Bar geometry for the FEM . . . . . . . . . . . . . . . . . . . . . . . . . . . 84

\begin{tabular}{lll}
\hline 5 & Active Anti-roll Complete Model & 87
\end{tabular} 
5.1 Active anti-roll bar . . . . . . . . . . . . . . . . . . . . . 87

$5.1 .1 \quad$ Non-conservative work $W_{n c} \ldots \ldots \ldots$. . . . . . . . . 88

$5.1 .2 \quad$ Equations of motion $\ldots \ldots \ldots \ldots$. . . . . . . . . . . . 89

5.1 .3 Solution . . . . . . . . . . . . . . . . . . . 94

5.2 Lateral acceleration as a disturbance $\ldots \ldots \ldots \ldots$

5.3 Bar geometry for the FEM $\ldots \ldots \ldots$. . . . . . . . . . . . . . . . . . . . . . . . . 99

5.4 Parameters . . . . . . . . . . . . . . . . . . . . . 100

5.5 Validation . . . . . . . . . . . . . . . . . . . . . 102

\begin{tabular}{lll}
\hline & Control design & 105
\end{tabular}

6.1 Representations of uncertainty . . . . . . . . . . . . . . . 105

6.2 Performance and robustness of feedback systems . . . . . . . . . . . . . . 107

6.3 The nominal case . . . . . . . . . . . . . . . . . . . . . . . . . . . . . 108

6.3 .1 Tracking of reference signal . . . . . . . . . . . . . . . 108

6.3 .2 Disturbance rejection . . . . . . . . . . . . . . . . . . 109

$6.3 .3 \quad$ Measurement error rejection . . . . . . . . . . . . . . . . . . . . 109

6.4 The real case . . . . . . . . . . . . . . . . . . . . . . 110

6.4 .1 Robust stability . . . . . . . . . . . . . . . . . 111

6.4 .2 Performance . . . . . . . . . . . . . . . . . . . . . . . . . . . 112

6.5 LQG/LTR control method . . . . . . . . . . . . . . . . . . . . 113

6.5 .1 Quadratic cost function . . . . . . . . . . . . . 113

6.5 .2 Solution of the LQG problem . . . . . . . . . . . . . . . . . . 114

6.5.2.1 The Kalman filter . . . . . . . . . . . . . . . . . . . . . 114

$6.5 .2 .2 \quad$ The LQR regulator . . . . . . . . . . . . . . . . . . . . . 115

6.5 .3 LQG analysis . . . . . . . . . . . . . . . . . . . 116

6.5 .4 LTR - loop transfer recovery . . . . . . . . . . . . . . . . . . . 116

$6.6 \quad H_{\infty}$ loop-shaping method $\ldots \ldots \ldots \ldots$. . . . . . . . . . . . . . . . . . 
6.6 .1 Robust stabilization . . . . . . . . . . . . . . . . 119

6.6 .2 The loop-shaping design procedure . . . . . . . . . . . . . . . . 121

$\begin{array}{lll}7 & \text { Simulation results } & 125\end{array}$

7.1 Design objectives . . . . . . . . . . . . . . . . . . . . . 125

7.2 Model reduction and uncertainty model . . . . . . . . . . . . . . . 126

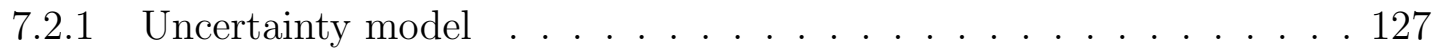

7.3 LQG/LTR controller . . . . . . . . . . . . . . . . . . . . . . . . . . . . . . 129

$7.4 \quad H_{\infty}$ loop-shaping controller . . . . . . . . . . . . . . . 133

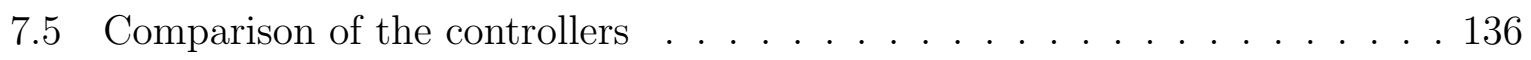

$7.5 .1 \quad$ Step steer test $\ldots \ldots \ldots \ldots$. . . . . . . . . . . . . 137

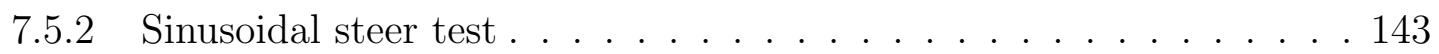

$7.5 .3 \quad$ Lane-change maneuver . . . . . . . . . . . . . . . . . . . . 147

$\begin{array}{lll}8 & \text { Conclusion } & 155\end{array}$

\begin{tabular}{ll}
\hline References & 157
\end{tabular} 


\section{INTRODUCTION}

\subsection{Published work}

Prior to the presentation of this dissertation, work relevant to development was presented by PAES J. H .; COLÓN D. (2018) at the Congresso Brasileiro de Automática (CBA) 2018.

This paper summarizes the part of the contents presented in this dissertation, in which only an LQG controller is applied to the anti-roll system consisting of an active flexible bar. The potential reduction of rollover accidents is evident by the results that show significant reduction to the roll angle of the car when it is submitted to cornering maneuvers.

\subsection{Motivation}

The composition of the current automotive fleet consists of $36 \%$ of light trucks, minivans and sport utility vehicles (SUVs). Unfortunately, the rate of fatal rollovers for pickups is twice that for passenger cars and the rate for SUVs is almost three times that of passenger cars. While rollover affects about $31 \%$ percent of passenger cars involved in crashes with occupant fatalities, it accounts for $51 \%$ of passenger cars's occupant fatalities in single crashes. These statistics are from 2016 and motivate a very real opportunity to improve passenger car's safety by preventing rollover via active stability control (NHTSA, 2016).

Active anti-roll systems allow the control of the roll angle and thus improves the vehicle stability, especially when turning or moving on slopping ground (JAMIL; ABDULLAH, 2010). A rollover is a type of vehicle crash in which a vehicle tips over onto its side or roof. Vehicle rollovers are divided into two categories: tripped and untripped. Tripped rollovers are caused by forces from an external object, such as a curb or a collision with another vehicle. Untripped crashes are the result of steering input, speed, and friction with the 
ground (PHANOMCHOENG; RAJAMANI, 2012). Independently of these categories, rollovers have a higher fatality rate than any other type of vehicle collision.

Untripped rollovers occur when cornering forces take out the stability of the vehicle. When a vehicle rounds a corner, the cornering forces acting on it are the tire forces on the ground under influence of the frictional surface (centripetal force), the inertial effects (centrifugal force) and the weight force, as seen in Figure 1. The tire force push the vehicle towards the center of the curve and acts at the ground level, below the c.g.. The force of inertia acts horizontally through the vehicle's c.g. away from the center of the turn. These two forces lead the vehicle to roll towards the outside of the curve. The weight force due to the gravity acts downward through the c.g. in the opposite direction. When the tire and inertial forces are enough to overcome the force of gravity, the vehicle starts to turn over. This type of rollover is more likely to occur in vehicles with a higher center of gravity than most passenger vehicles, e.g. SUV. The surface type includes pavement surfaces plus gravel, grass, dirt, etc (CARLSON; GERDES, 2003).

Figure 1: Cornering forces acting on the vehicle under curved trajectory.

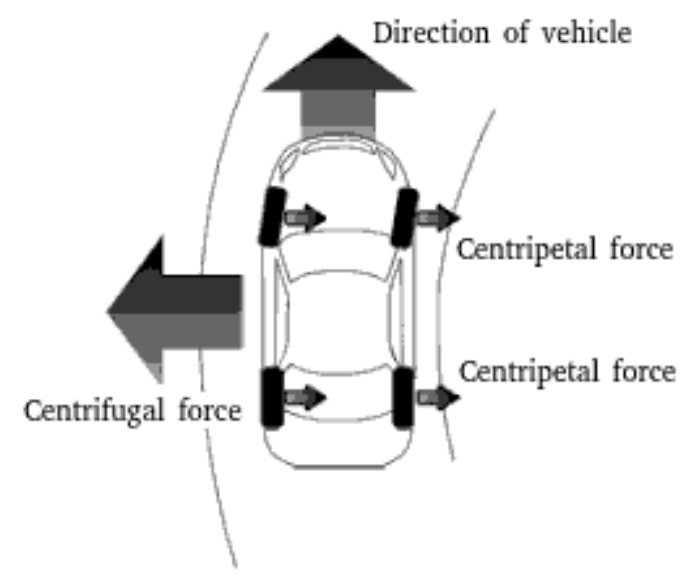

Source: https://www.rimsdealer.com/content/.

In addition to finding dynamic models of vehicle's roll motion, this study is further focused on finding hybridized dynamic models with FEM modeled flexible anti-roll bars that are well suited to the design of controllers. These controllers are increasingly used to prevent the onset of rollover. Hence, a goal of this work is to understand the linear vehicle dynamics when flexible anti-roll bars are attached to the suspension system, and afterward, to design an active anti-roll system able to mitigate the rollover occurrence. Therefore only linear models are considered. Suitability for controller synthesis is determined by a number of factors including the accuracy of the model for moderate steering 
inputs (e.g. linear tire behavior and small roll angles) and the order and simplicity of the dynamic model (model order reduction), as FEM approach produces models with large number of degrees of freedom (d.o.f.) that causes numerical difficulties in dynamic analysis and control design.

\subsection{Literature survey}

Carlson and Gerdes uses Model Predictive Control (MPC) theory to develop a framework for automobile stability control. The framework is then demonstrated with a roll mode controller which seeks to actively limit the peak roll angle of the vehicle while simultaneously tracks the driver's yaw rate command (CARLSON; GERDES, 2003).

Cameron and Brennan presents results of an initial investigation into models and control strategies suitable to prevent vehicle rollover due to untripped driving maneuvers. Outside of industry, the study of vehicle rollover including both experimental validation and practical controller design is limited. The researcher interested in beginning the study on rollover dynamics and control is left with the challenging task of identifying suitable vehicle models from the literature, comparing these models with experimental results, and determining suitable parameters for the models. Cameron and Brennan addresses these issues via experimental testing of published models. Parameter estimation data based on model fits is presented, with comments given on the validity of different methods. Experimental results were presented and compared to the output predicted by the various models in both the time and frequency domain (CAMERON; BRENNAN, 2005).

A pair of MPC controllers capable of modifying the nominal roll dynamics of a vehicle through control of the planar vehicle dynamics are presented by Craig and Gerdes. Each of these controllers are based on a linear model of the vehicle. One controller utilizes differential drive of the rear axle to modify the planar motion, while the other uses rear-wheel-steering. Results from simulations of a nonlinear vehicle model executing maneuvers with each of the controllers demonstrate that the differential-drive technique results in significant lateral-longitudinal tire force coupling and saturation that degrades the validity of the internal model used for controller. The rear-wheel-steering controller commands very small steering angles, retaining the validity of the model and showing better performance for the desired roll control task (CRAIG; GERDES, 2010).

Kim and Park presents the design of an active roll controller for a vehicle and an experimental study using the electrically actuated roll control system. Firstly, parameter 
sensitivity analysis is performed based on the 3 DOF linear vehicle model. The controller is designed in the framework of lateral acceleration control and gain-scheduling control scheme considering the varying parameters induced by laden and running vehicle condition. In order to investigate the feasibility of an active roll control system, experimental work is performed using a hardware-in-the-loop setup, which has been constructed using the devised electrically actuated system and a full vehicle model with tire characteristics. The performance was evaluated by experiment using the devised hardware-in-the-loop setup under the conditions of steering maneuvers and parameter variations (KIM; PARK. 2004).

Bharane et al. perform a finite element analysis of an anti-roll bar, including a preprocessing, analysis, post processing, and analyzing the FEA results by using a commercial software. The effects of anti-roll bar design parameters on final anti-roll bar properties are evaluated by performing sample analysis. They show that the most effect of using hollow section is the reduction in mass of the bar and that by changing the parameters of the bar, it properties can be improved.

A method for designing a controller that uses an active anti-roll bar (AARB) and an electronic stability program (ESP) for rollover prevention is presented by Yim et al. ESP with longitudinal speed control (LSC) can carry out active braking to reduce vehicle speed and lateral acceleration to prevent a rollover. To enhance the rollover prevention capability of the ESP, an AARB was adopted. The controller for the AARB was designed based on linear quadratic (LQ) static output feedback (SOF) control methodology, which attenuates the effect of lateral acceleration on the roll angle and roll rate by control of the suspension stroke and the tire deflection of the vehicle. Although this AARB significantly increases ride comfort and rollover prevention, it has a drawback - the vehicle loses its maneuverability. Therefore, the ESP with LSC is used to overcome this drawback (YIM; JEON; YI, 2012).

In active rollover prevention systems, a real-time rollover index, which indicates the likelihood of the vehicle to rollover, is used. Rajamani et al. focuses on state and parameter estimation for reliable computation of the rollover index. Two key variables that are difficult to measure and play a critical role in the rollover index are found to be the roll angle and the height of the center of gravity of the vehicle. Algorithms are developed for real-time estimation of these variables (RAJAMANI et al., 2011). 


\subsection{Anti-roll systems}

\subsubsection{Anti-roll bars}

Anti-roll bars are used to increase the vehicle's safety during curved trajectories by reducing the roll angle during cornering and to improve the driving behavior and comfort by positively influencing the self-steering behavior of the vehicle's chassis AMMON, 2013). Their working principle is based on increasing the rolling stiffness of the vehicle by coupling the forces between the two tires of a single axle during different compression travels. This results in a reduction of the normal force difference between the two tires on the relevant axle (SCHRAMM MANFRED HILLER, 2014).

Anti-roll bars provide two main functions. The first function is the reduction of body lean. Body lean (or body roll) happens when the car turn into a corner. As the car begins to turn, its weight is thrown to the outside of the corner, causing the vehicle to roll in that direction. The reduction of body lean is dependent on the total roll stiffness of the vehicle. Roll stiffness stands for the difficulty for the car to roll. Increasing the total roll stiffness of a vehicle does not change the steady state total load (weight) transfer from the inside wheels to the outside wheels, it only reduces body lean. The static stability factor (SSF) is related to the total lateral load transfer and is defined as:

$$
S S F=\frac{l}{2 h},
$$

where $l$ and $h$ are the track width and height of the c.g. of the vehicle measured from the ground respectively, as shown in Figure 2 (RAJAMANI, 2012). The higher the SSF value, the greater the stability of the vehicle in relation to the roll.

Figure 2: Parameters for vehicle's static stability factor.

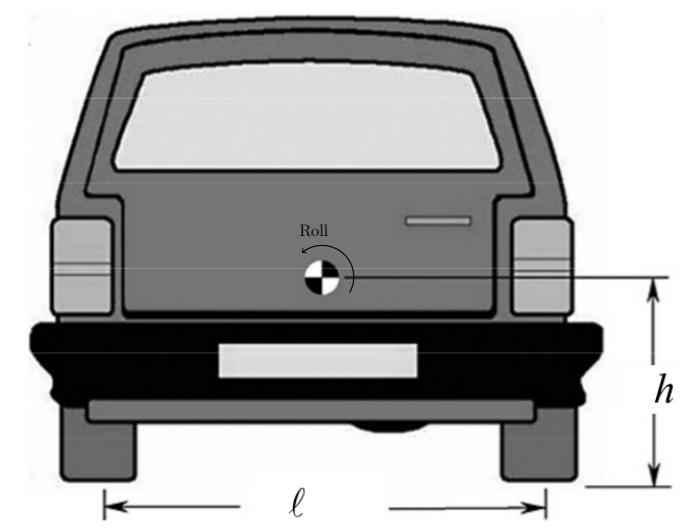

Source: (RAJAMANI, 2012). 
The other function of anti-roll bars is to tune the handling balance of a car. Understeer or oversteer behavior can be tuned out by changing the proportion of the total roll stiffness that comes from the front and rear axles. Increasing the proportion of roll stiffness at the front increases the proportion of the total load transfer. In this way, the reaction of the front axle decreases the proportion that the rear axle reacts to. In general, this makes the outer front wheel run at a comparatively higher slip angle, and the outer rear wheel to run at a comparatively lower slip angle, which is an understeer effect. Increasing the proportion of roll stiffness at the rear axle has the opposite effect and decreases understeer (GILLESPIE, 1997).

Therefore, an anti-roll bar is intended to actuate each side of the vehicle's suspension in order to reduce the roll angle of the vehicle when running in curves, essentially counteracting the centrifugal forces of the vehicle. With the bar removed, the vehicle's wheels can tilt away by much larger distances. Although there are many variations in design, the common function is to force the opposite wheel's shock absorber, spring or suspension rod to lower, or rise, to a similar level as the other wheel.

\subsubsection{Passive anti-roll bars}

A passive anti-roll bar is usually a bent, U-shaped torsion bar spring, as shown in Figure 3. It is mounted on the vehicle chassis using rubber sleeves at A,A' (see Figure 4), allowing a rotational d.o.f. at this connection. Their ends B, B' are connected to the two sides of the wheel suspension of the concerned axle. At different deflections of the spring of the two tires, the levers $b$ are subject to different displacements, resulting in a twist of the torsion beam, and thus a corresponding torsion torque on the chassis (SCHRAMM MANFRED HILLER, 2014). 
Figure 3: Passive anti-roll bar placed in a vehicle's suspension system.

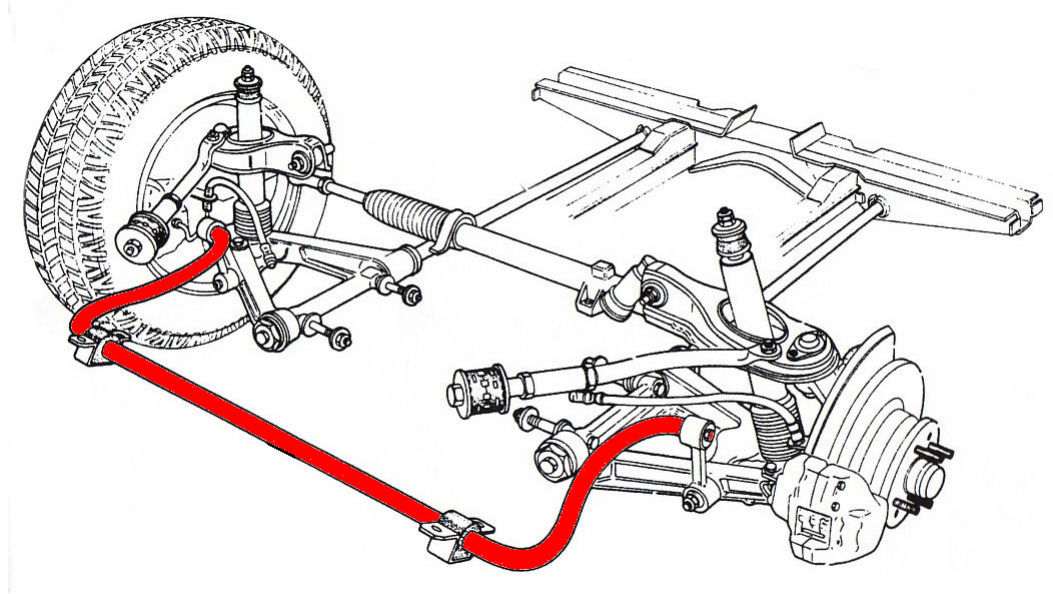

Source: https://en.wikipedia.org/wiki/Anti-roll_bar.

Figure 4: Passive anti-roll bar schematics.

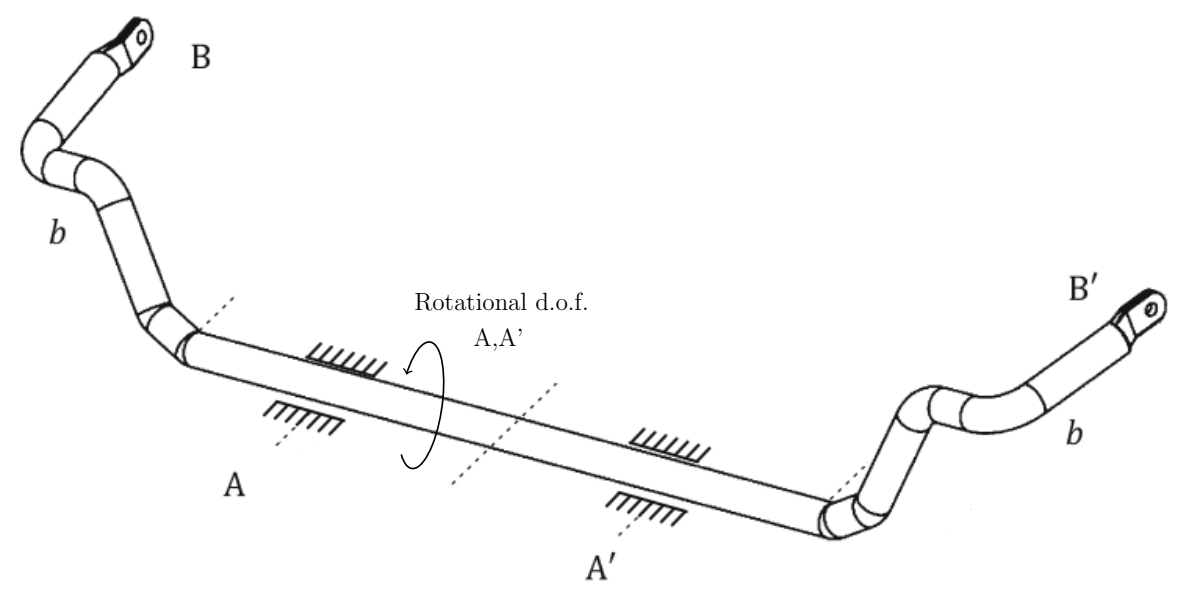

Source: (RAJAMANI, 2012).

\subsubsection{Active anti-roll bars}

Over the past few years, active anti-roll bars have been implemented as actuators in order to achieve rolling stabilization and to influence the vehicle's dynamics (ÖTTGEN, 2005). Great brands like Audi, Bentley and Lamborghini SUVs, Mercedes and Rolls Royce has already include this system into yours luxury cars, like Audi A7 model.

Looking the rolling performance improvement, the passive anti-roll bar is basically split into two bars and the free ends are then usually re-linked using an electrical actuator, as shown in Figure 5. Instead of the passive anti-roll bar, which is dependent on the torsion 
of the anti-roll bar, an active torque $M$ is created (Figure 6), which is supported by the wheel carrier over the anti-roll bar connecting link, and through the reaction forces, a torque is applied onto the vehicle chassis over the vehicle's longitudinal axis. Thus, roll stabilization is realizable and the self-steering characteristics of the vehicle can be influenced through the partitioning of the applied torques in the front and rear axle. In models the actuator can usually be assumed to be ideal, even though the applied torques are naturally constrained as control variables (SCHRAMM MANFRED HILLER, 2014).

Figure 5: Active anti-roll bar.

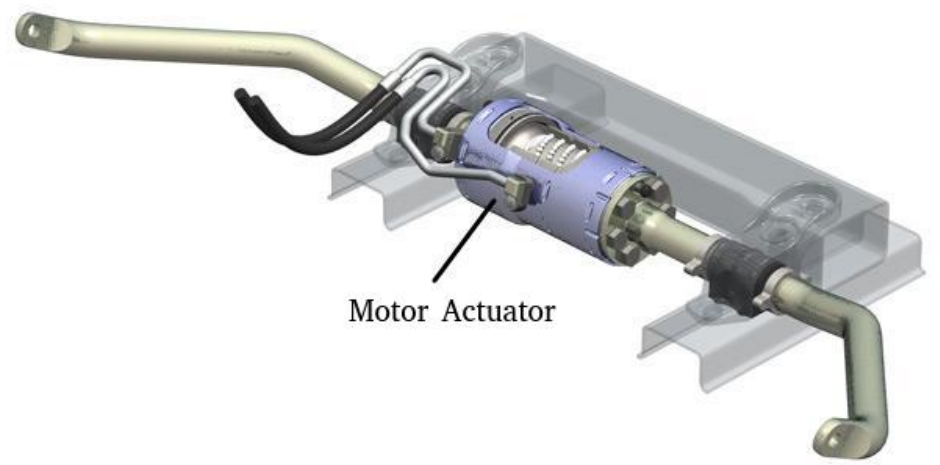

Source: https://www.bwigroup.com/product/asbs-active-stabilizer-bar-systems/.

Figure 6: Active anti-roll bar schematics.

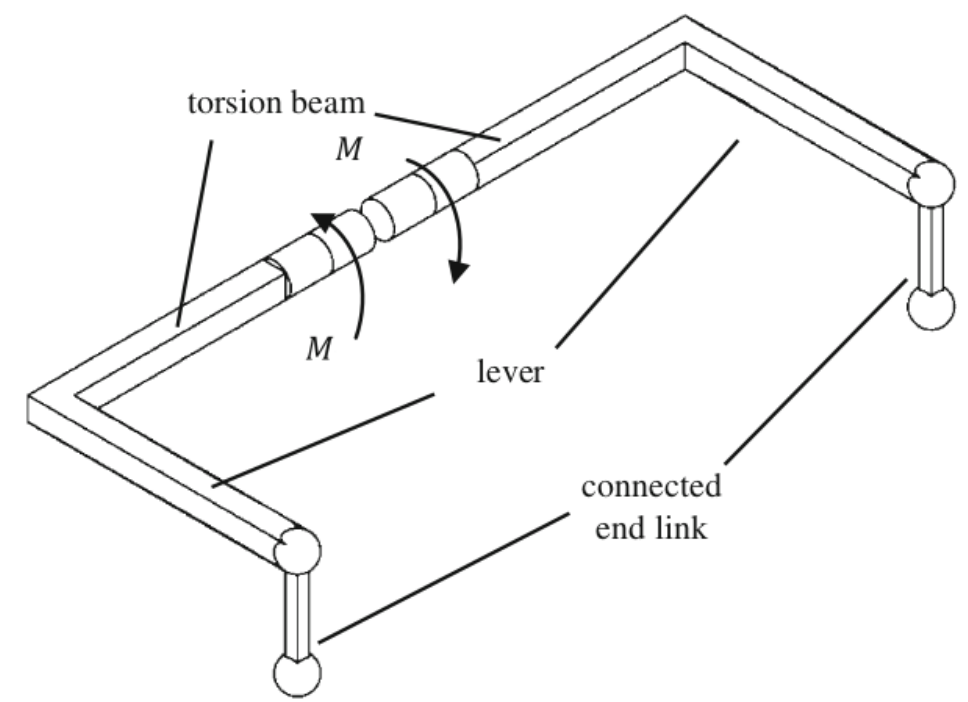

Source: (RAJAMANI, 2012). 


\subsection{Structure of the thesis}

The remainder of the work is organized as follows: Chapter 2 presents the theory fundamentals necessary for this study. The basic dynamic equations of the vehicle's lateral dynamics are presented in Chapter 3 . Chapters 4 and 5 presents the mathematical approach of the FEM models for the passive and active systems, respectively. Control design of the proposed methods and the respective robustness analysis are presented in Chapter 6. Chapter 7 exhibits the results taken from three models: roll dynamics model without anti-roll system, with passive and active anti-roll bar system as well. Three maneuvers, step, sinusoidal and lane-change tests, are considered in order to compare the models and the designed controllers. Finally, Chapter 8 presents the conclusions from this study and the next possible steps. 


\section{THEORY FUNDAMENTALS}

\subsection{Analytical mechanics}

\subsubsection{The principle of virtual work}

This part was made according to the chapter two of (MEIROVITCH, 1990). The fundamentals of the principle of virtual works are centered on the static equilibrium of a system. The main idea in the principle is carrying out the transition from Newtonian to Lagrangian mechanics.

Consider a system composed of $N$ particles and let the $\mathbf{R}_{i}(i=1,2, \ldots, N)$ to be the resultant force acting on particle $i$. If the system is in equilibrium, $\mathbf{R}_{i}=\mathbf{0}$ and, consequently, the dot product $\mathbf{R}_{i} \cdot \delta \mathbf{r}_{i}=0$, where $\mathbf{R}_{i} \cdot \delta \mathbf{r}_{i}$ represents the virtual work performed by the force $\mathbf{R}_{i}$ through the virtual displacement $\delta \mathbf{r}_{i}$. The virtual displacements are not necessarily actual displacements, but it experiences infinitesimal changes in the coordinates of the respective system. Although the virtual displacements satisfies the system constraints, they are arbitrary. Furthermore, they are imagined to take place simultaneously, which implies that the virtual displacements are the result of imagining the system in a little displaced position, not taking into account the time. Since the $\mathbf{R}_{i} \cdot \delta \mathbf{r}_{i}$ is zero for every particle, the virtual work for the whole system vanishes, and the following equation can be written:

$$
\delta W=\sum_{i=1}^{N} \mathbf{R}_{i} \cdot \delta \mathbf{r}_{i}=0 .
$$

Including the action of the constraints forces on the system, then the resultant force $\mathbf{R}_{i}$ can be written as:

$$
\mathbf{R}_{i}=\mathbf{F}_{i}+\mathbf{F}_{i}^{\prime}, \quad i=1,2, \ldots, N,
$$

where $\mathbf{F}_{i}$ are externally applied forces and $\mathbf{F}_{i}^{\prime}$ denote the constraints forces. Considering 
the substitution of the resultant forces presented in Equation 2.2 into Equation 2.1 yields:

$$
\delta W=\sum_{i=1}^{N} \mathbf{F}_{i} \cdot \delta \mathbf{r}_{i}+\sum_{i=1}^{N} \mathbf{F}_{i}^{\prime} \cdot \delta \mathbf{r}_{i}=0 .
$$

Constraint forces, on the other hand, perform no work because virtual displacements never act in the parallel direction of the constraint. Therefore, the sum of the components $\mathbf{F}_{i}^{\prime}$ in Equation 2.3 is equal to zero, and the equation is rewritten as:

$$
\delta W=\sum_{i=1}^{N} \mathbf{F}_{i} \cdot \delta \mathbf{r}_{i}=0,
$$

then, the work performed by the external forces through virtual displacements compatible with the system constraints is zero. This is the principle of virtual work. In a general way, the principle of virtual work is expressed in terms of generalized coordinates, which can describe the configuration of the system relative to some reference configuration. The generalized principle of virtual work is:

$$
\delta W=\sum_{k=1}^{n} \mathbf{Q}_{k} \cdot \delta \mathbf{q}_{k}=0,
$$

where $\delta \mathbf{q}_{k}(k=1,2, \ldots, n)$ are virtual generalized displacements (that can be linear or angular position). Consequently, $\mathbf{Q}_{k}(k=1,2, \ldots, n)$ are known as generalized forces.

\subsubsection{D'Alembert's principle}

As said in the previous section, the center point of the principle of virtual work is the static equilibrium state of a system. This concept can be extended to dynamical systems by means of a principle attributed to d'Alembert. System equations of motion can be obtained through the D'Alembert's principle. The transition from the Newtonian mechanics to Lagrangian mechanics is presented next.

Let's states the Newton's second law for a typical particle $i$, with mass $m_{i}$. It can be written in the form:

$$
\mathbf{F}_{i}-m_{i} \ddot{\mathbf{r}}_{i}=\mathbf{0}, \quad i=1,2, \ldots, N
$$

which can be written as:

$$
\left(\mathbf{F}_{i}-m_{i} \ddot{\mathbf{r}}_{i}\right) \cdot \delta \mathbf{r}_{i}=0, \quad i=1,2, \ldots, N
$$


For a system composed by $N$ particles, the total equation is written as:

$$
\sum_{i=1}^{N}\left(\mathbf{F}_{i}-m_{i} \ddot{\mathbf{r}}_{i}\right) \cdot \delta \mathbf{r}_{i}=0
$$

which is referred to as the generalized principle of d'Alembert.

\subsubsection{Hamilton's principle}

In contrast with the static case, the virtual work is not zero in the dynamic case. In order to deal with, it is considered the following variation:

$$
\frac{d}{d t}\left(\dot{\mathbf{r}}_{i} \cdot \delta \mathbf{r}_{i}\right)=\ddot{\mathbf{r}}_{i} \cdot \delta \mathbf{r}_{i}+\dot{\mathbf{r}}_{i} \cdot \delta \dot{\mathbf{r}}_{i}=\ddot{\mathbf{r}}_{i} \cdot \delta \mathbf{r}_{i}+\delta\left(\frac{1}{2} \dot{\mathbf{r}}_{i} \cdot \dot{\mathbf{r}}_{i}\right),
$$

and:

$$
\ddot{\mathbf{r}} i \delta \mathbf{r}_{i}=\frac{d}{d t}\left(\dot{\mathbf{r}}_{i} \cdot \delta \mathbf{r}_{i}\right)-\delta\left(\frac{1}{2} \dot{\mathbf{r}}_{i} \cdot \dot{\mathbf{r}}_{i}\right) .
$$

Multiplying Equation 2.10 by $m_{i}$ and summing up over the entire system leads to:

$$
\sum_{i=1}^{N} m_{i} \ddot{\mathbf{r}}_{i} \cdot \delta \mathbf{r}_{i}=\sum_{i=1}^{N} m_{i} \frac{d}{d t}\left(\dot{\mathbf{r}}_{i} \cdot \delta \mathbf{r}_{i}\right)-\delta T
$$

where $T$ is the system's kinetic energy. By Equations 2.4 and 2.11 into Equation 2.8 yields:

$$
\delta T+\delta W=\sum_{i=1}^{N} m_{i} \frac{d}{d t}\left(\dot{\mathbf{r}}_{i} \cdot \delta \mathbf{r}_{i}\right)
$$

Through the solution of $\mathbf{r}_{i}=\mathbf{r}_{i}(t)(i=1,2, \ldots, N)$ in the chosen configuration space, (MEIROVITCH, 1990) shows that, by integrating in both sides, the Equation 2.12 can be written as:

$$
\int_{t_{1}}^{t_{2}}(\delta T+\delta W) d t=0, \quad \delta \mathbf{r}_{i}\left(t_{1}\right)=\delta \mathbf{r}_{i}\left(t_{2}\right)=\mathbf{0}, \quad i=1,2, \ldots, N
$$

which is known as the extended Hamilton's principle. The $\delta \mathbf{r}_{i}\left(t_{1}\right)$ and $\delta \mathbf{r}_{i}\left(t_{2}\right)$ are the space variations at time $t_{1}$ and $t_{2}$, respectively.

The extended Hamilton's principle can be written in a slightly more convenient form. To this end, the virtual work is divided into two parts, one due to the conservative forces and the other due to non-conservative forces, or:

$$
\delta W=\delta W_{c}+\delta W_{n c}
$$


It is possible to write for the virtual work due to conservative forces the following:

$$
\delta W_{c}=-\delta V
$$

where $V$ is the potential energy associated to the conservative forces. Hence, introducing the Lagrangian $L=T-V$ and considering Equations 2.14 and 2.15, the extended Hamilton's principle, Equation 2.16, can be rewritten in the form:

$$
\int_{t_{1}}^{t_{2}}\left(\delta L+\delta W_{n c}\right) d t=0, \quad \delta \mathbf{r}_{i}\left(t_{1}\right)=\delta \mathbf{r}_{i}\left(t_{2}\right)=\mathbf{0}, \quad i=1,2, \ldots, N
$$

\subsection{Dynamic of flexible structures}

\subsubsection{Flexible structures}

This part was made according to the chapters two, three and four of (GAWRONSKI, 2006). A flexible structure is a system of infinite size, and after realizing spatial discretization, it is transformed into a finite dimension system. Then, the structure shall be defined as a finite-dimensional, controllable, and observable linear system with complex poles and with small damping. A linear system that fits the above definition of flexible structure is represented by the second-order matrix differential equation:

$$
M \ddot{v}+D \dot{v}+K v=F u, \quad y=C_{v} v+C_{\dot{v}} \dot{v}
$$

where $v$ is the $n \times 1$ displacement vector, $u$ is the $k \times 1$ input vector, $y$ is the $r \times 1$ output vector, $M$ is the $n \times n$ mass matrix, $D$ is the $n \times n$ damping matrix, $K$ is the $n \times n$ stiffness matrix, the input matrix $F$ is $n \times k$, the output displacement matrix $C_{v}$ is $r \times n$, and the output velocity matrix $C_{\dot{v}}$ is $r \times n$. The number $n$ is the number of d.o.f. of the system which is the linearly independent coordinates describing the finite-dimensional structure, $r$ is a number of outputs, and $k$ is a number of inputs. The mass matrix is typically positive definite, and the stiffness and damping matrices are typically positive semi-definite. The above equation is obtained, for example, as a result of finite-element modeling, or as a result of system identification.

\subsubsection{State-space representations}

In order to make the system suitable for control analysis and design purposes, the flexible structure described in Equation 2.17 must be transformed into the state-space 
form:

$$
\begin{aligned}
& \dot{x}=A x+B u, \\
& y=C x .
\end{aligned}
$$

The matrices $(A, B, C)$ is the state-space representation of the system, and $x$ is the state vector. The state representation and the state vector are not unique, in the sense that different state representations (coordinate systems) give the same input-output relationship. It is common to introduce a new state variable, $\hat{x}$, such that $x=R \hat{x}$ and $R$ is non-singular, and a new state representation $(\hat{A}, \hat{B}, \hat{C})$ is obtained:

$$
\hat{A}=R^{-1} A R, \quad \hat{B}=R^{-1} B, \quad \hat{C}=C R .
$$

Although input-output relations remain invariant, the choice of the state representation has a significant impact in the control design. Some representations have useful physical interpretations, others are more suitable for analysis and design. For this study, the interest is primarily to use the balanced representation, which has important properties concerning the controllability and observability. The balanced representation will be present in the next section.

In order to obtain a state representation for Equation 2.17, it is rewritten as follows, considering the mass matrix non-singular:

$$
\ddot{v}+M^{-1} D \dot{v}+M^{-1} K v=M^{-1} F u, \quad y=C_{v} v+C_{\dot{v}} \dot{v} .
$$

Define the state vector $x^{T}=\left[\begin{array}{ll}v & \dot{v}\end{array}\right]^{T}$, in which the first component is the system displacement, and the second component is the system velocity. In this case, after elementary manipulations, one obtains the following state-space representation:

$$
A=\left[\begin{array}{cc}
0 & I \\
-M^{-1} K & -M^{-1} D
\end{array}\right], \quad B=\left[\begin{array}{c}
0 \\
M^{-1} F
\end{array}\right], \quad C=\left[\begin{array}{ll}
C_{q} & C_{v}
\end{array}\right],
$$

where $A$ is $n_{t} \times n_{t}, B$ is $n_{t} \times k$, and $C$ is $r \times n$. The dimension of the state space model is twice the number of d.o.f. of the system $n$, i.e., $n_{t}=2 n$.

\subsubsection{Balanced open-loop systems}

Controller design relies heavily on the plant controllability and observability properties. Heuristically, controllability is a property of a plant input to excite the total system 
dynamics (i.e., a property that all states are excited by the input actuators). Observability, on the other hand, is a property of the output and includes total system dynamics (i.e., the property that all states are sensed by the output sensors). Individually, neither controllability nor observability provide sufficient information for a controller design. For example, if parts of the states are weakly controllable (weakly influenced by the plant input), it is impossible to decide on the controller configuration, since the same states could be strongly observable by the output sensors. Similarly, the weakly observable states, which are weakly reflected in the plant output, could be strongly excited by the input actuators. However, if a set of the stable states is weakly controllable and observable at the same time (weakly influenced by the plant input and weakly sensed in the plant output), it can be ignored in the controller design. The joint controllability and observability properties of the system are characterized by the Hankel singular values, and this simple idea was developed by Moore (MOORE, 1981).

Instead of using the controllability and observability properties, each state variable can be characterized by a cost. In this work, the cost of a state variable is given by the Hankel singular values and indicates its participation in the system output. If the participation is strong, the cost is high, and vice versa.

There are two disadvantages when the state variable's cost is used. As said early, the cost only evaluates a state at the system output, while the input seems to be ignored. Additionally, if two states are highly coupled, their individual cost does not reflect their actual contribution in the output. Balanced representation of flexible structures is useful to overcome these drawbacks, in which the state components are weakly coupled and their cost can be easily evaluated (SKELTON, 1980).

\subsubsection{Observability and controllability grammians}

The controllability and observability grammians are the convenient form of characterization of a system controllability and observability properties. They are defined in the following. Consider a linear, observable and controllable system with state-space representation $(A, B, C)$. The system is assumed stable unless otherwise stated. It can be, of course, a flexible structure, but not necessarily. The controllability and observability grammians are defined as follows:

$$
\begin{aligned}
& W_{c}(t)=\int_{0}^{t} \mathrm{e}^{A \tau} B B^{T} \mathrm{e}^{A^{T} \tau} d \tau, \\
& W_{o}(t)=\int_{0}^{t} \mathrm{e}^{A^{T} \tau} C^{T} C \mathrm{e}^{A \tau} d \tau .
\end{aligned}
$$


Alternatively, they can be determined from the following matrix differential equations:

$$
\begin{aligned}
& \dot{W}_{c}=A W_{c}+W_{c} A^{T}+B B^{T}, \\
& \dot{W}_{o}=A^{T} W_{o}+W_{o} A+C^{T} C .
\end{aligned}
$$

The stationary solutions of Equation 2.23 are obtained in the limiting case of $t \rightarrow \infty$. In this case, for a stable system one obtains $\dot{W}_{c}=\dot{W}_{o}=0$, and the grammians are determined from the following Lyapunov equations:

$$
\begin{aligned}
& A W_{c}+W_{c} A^{T}+B B^{T}=0, \\
& A^{T} W_{o}+W_{o} A+C^{T} C=0,
\end{aligned}
$$

where for stable $A$ the solutions of Equation 2.24 are positive definite. The grammians depend on the system coordinates (state space representation), and for a linear transformation of state, $\tilde{x}=R x$, they are transformed as follows:

$$
\begin{aligned}
& \tilde{W}_{c}=R^{-1} W_{c} R^{-T}, \\
& \tilde{W}_{o}=R^{T} W_{o} R .
\end{aligned}
$$

However, the eigenvalues of the grammian product are invariant under linear transformation, since:

$$
\lambda_{i}\left(\tilde{W}_{c} \tilde{W}_{o}\right)=\lambda_{i}\left(R^{-1} W_{c} R^{-T} R^{T} W_{o} R\right)=\lambda_{i}\left(R^{-1} W_{c} W_{o} R\right)=\lambda_{i}\left(W_{c} W_{o}\right)
$$

\subsubsection{Balanced representation}

The system is open-loop balanced, if its controllability and observability grammians are equal and diagonal, as defined by Moore in (MOORE, 1981):

$$
W_{c}=W_{o}=\Gamma^{2}, \quad \Gamma=\operatorname{diag}\left(\gamma_{1}, \ldots, \gamma_{n}\right), \quad \gamma_{i} \geq 0, \quad i=1, \ldots, n,
$$

where $\gamma_{i}$ is the $i$ th Hankel singular value of the system. The transformation $R$ to the balanced representation, such that $\left(A_{b}, B_{b}, C_{b}\right)=\left(R^{-1} A_{b} R, R^{-1} B_{b}, C_{b} R\right)$, is determined as follows:

$$
R=P U \Gamma^{-1}, \quad R^{-1}=\Gamma^{-1} V^{T} Q,
$$

where the matrices $\Gamma, V, U$ are obtained from the singular value decomposition of the matrix $H$ :

$$
H=V \Gamma^{2} U^{T}
$$


where $H$ is obtained as a product of the matrices $P$ and $Q$ :

$$
H=Q P
$$

and $P, Q$ are obtained from the decomposition of the controllability and observability grammians respectively:

$$
W_{c}=P P^{T}, \quad W_{o}=Q^{T} Q
$$

This decomposition can be achieved by the Cholesky, or singular value decomposition.

\subsubsection{Model reduction}

Model reduction is a part of the dynamic analysis of flexible structures. Typically, a model with large number of degrees of freedom causes numerical difficulties in the dynamic analysis and has a high computational cost. Additionally, if one takes into account that the complexity of a controller depends on the plant order, then it is not difficult to see that a full-order controller for a high-order plant is hardly implementable. Thus the reduction of system order solves the problem, assuming that the reduced model acquires the essential properties of the full-order model (GAWRONSKI, 2006).

A reduced-order model of a linear system is obtained for this work by truncating appropriate states in the balanced or modal coordinates. In order to do this let the state vector $x$ of the system in the balanced representation be partitioned as follows, $x^{T}=\left[x_{r} x_{t}\right]^{T}$, and the state triple $(A, B, C)$ be partitioned accordingly:

$$
A=\left[\begin{array}{cc}
A_{r} & A_{r t} \\
A_{t r} & A_{t}
\end{array}\right], \quad B=\left[\begin{array}{c}
B_{r} \\
B_{t}
\end{array}\right], \quad C=\left[\begin{array}{ll}
C_{r} & C_{t}
\end{array}\right],
$$

where $x_{r}$ is a $k \times l$ vector and $x_{t}$ is an $(n-k) \times l$ vector. The balanced grammian is divided similarly:

$$
\Gamma^{2}=\left[\begin{array}{cc}
\Gamma_{r}^{2} & 0 \\
0 & \Gamma_{t}^{2}
\end{array}\right]
$$

The reduced model is obtained by deleting the last $n-k$ rows of $A, B$, and the last $n-k$ columns of $A, C$, i.e.:

$$
A_{r}=L A L^{T}, \quad B_{r}=L B, \quad C_{r}=C L^{T}, \quad L=\left[I_{k} 0\right] .
$$




\section{$2.3 \quad$ Finite element method}

In order to analyze an engineering system, a mathematical model is developed to describe the system. While developing the mathematical model, some assumptions are made for simplification. Finally, the governing mathematical expression is developed to describe the behavior of the system. The mathematical expression usually consists of differential equations and given conditions (BANG; KWON, 2000).

These differential equations are usually very difficult or impossible to solve explicitly. With the advent of high performance computers, it has become possible to solve such differential equations by numerical methods. Various numerical solution techniques have been developed and applied to solve numerous engineering problems in order to find their approximate solutions. Especially, the finite element method (or FEM) has been one of the major numerical solution techniques. One of the major advantages of the FEM is that a general purpose computer program can be easily developed to analyze various kinds of problems. In particular, any complex shape of problem domain with prescribed conditions can be handled with ease using the FEM (BANG; KWON, 2000).

The FEM requires division of the problem domain into many subdomains and each subdomain is called a finite element. Therefore, the problem domain consists of many finite element patches.

\subsubsection{Method of weighted residuals}

This part was made based on an example presented in chapter two of (BANG; KWON, 2000). The method of weighted residuals are useful to obtain approximate solutions to a differential governing equation. In order to explain the method, it is considered the following sample problem:

$$
\begin{array}{r}
\frac{d^{2} u}{d x^{2}}-u=x, \quad 0<x<1, \\
u(0)=0, \quad u(1)=0 .
\end{array}
$$

The first step in the method of weighted residuals is to assume a trial function which contains unknown coefficients to be determined later. For example, a trial function, $\tilde{u}=a x(1-x)$, is selected as an approximated solution to Equation 2.35. Here, ${ }^{\sim}$ denotes an approximate solution which is usually different from the exact solution. The trial 
function is chosen here such that it satisfies the boundary conditions (i.e., $\tilde{u}(0)=0$ and $\tilde{u}(1)=0$ ), and it has one unknown coefficient $a$ to be determined.

In general, the accuracy of an approximated solution is dependent upon proper selection of the trial function. In this example, a simple form of trial function is selected for the presented example to show the basic procedure of the methods of weighted residual. Once a trial function is selected, the residual is computed by substituting the trial function into the differential equation. That is, the residual $R$ becomes:

$$
R=\frac{d^{2} \tilde{u}}{d x^{2}}-\tilde{u}-x=-2 a-a x(1-x)-x
$$

Because $\tilde{u}$ is different from the exact solution, the residual does not vanish for all values of $x$ within the domain. The next step is to determine the unknown constant $a$ such that the chosen test function best approximates the exact solution. To this end, a test (or weighting) function $\omega$ is selected and weighted average of the residual over the problem domain is set to zero. That is:

$$
\begin{aligned}
I & =\int_{0}^{1} \omega R \mathrm{~d} x=\int_{0}^{1} \omega\left(\frac{d^{2} \tilde{u}}{d x^{2}}-\tilde{u}-x\right) \mathrm{d} x \\
& =\int_{0}^{1} \omega[-2 a-a x(1-x)-x] \mathrm{d} x=0 .
\end{aligned}
$$

The next step is to choose the test function. The resulting approximated solution differs depending on the test function. The methods of weighted residual can be classified based on how the test function is determined. Some of the methods of weighted residual are explained below.

1. Collocation Method. The Dirac delta function $\delta\left(x-x_{i}\right)$ is used as the test function, where the sampling point $x_{i}$ must be within the domain, $0<x_{i}<1$. In other words:

$$
\omega=\delta\left(x-x_{i}\right)
$$

Let $x_{i}=0.5$ and substitute the test function into the weighted residual, Equation 2.37 , to find $a=0.2222$. Then, the approximate solution becomes $\tilde{u}=0.2222 x(1-$ $x)$.

2. Least Squares Method. The test function is determined from the residual such that:

$$
\omega=\frac{d R}{d a}
$$


Applying Equation 2.39 into Equation 2.36 yields $\omega=-2-x(1-x)$. Substitution of the test function into Equation 2.37 results in $a=0.2305$. Then, $\tilde{u}=0.2305 x(1-x)$.

3. Galerkin's Method. For Galerkin's method, the test function comes from the chosen trial function. That is:

$$
\omega=\frac{d \tilde{u}}{d a}
$$

For the present trial function, $\omega=x(1-x)$. Applying this test function to Equation 2.36 gives $a=0.2272$ so that $\tilde{u}=0.2272 x(x-1)$. Comparison of these three approximate solutions to the exact solution at $x=0.5$ is provided in Table 1 . As seen in the comparison, all three methods result in reasonably accurate approximate solutions to Equation 2.35.

Table 1: Comparison of solution to Equation 2.35 at $x=0.5$.

\begin{tabular}{|c|c|c|c|}
\hline Exact Solution & Collocation & Least Squares & Galerkin \\
\hline 0.0566 & 0.0556 & 0.0576 & 0.0568 \\
\hline
\end{tabular}

Source: Author.

In the next chapter will be developed the mathematical model for lateral dynamics of a vehicle without anti-roll system. Moreover, the flexible anti-roll bar will be attached to the previous system. For this model is used the nomenclature: passive system, which any type of actuation is not used. 


\section{MATHEMATICAL MODEL: BASIC DYNAMIC EQUATIONS OF THE PASSIVE SYSTEM}

The Figure 7 represents the vehicle's model to roll dynamics with the passive anti-roll bar connected at each one of the suspensions. Later, this model will be more detailed.

Figure 7: Schematic model for the roll dynamics with passive anti-roll bar.

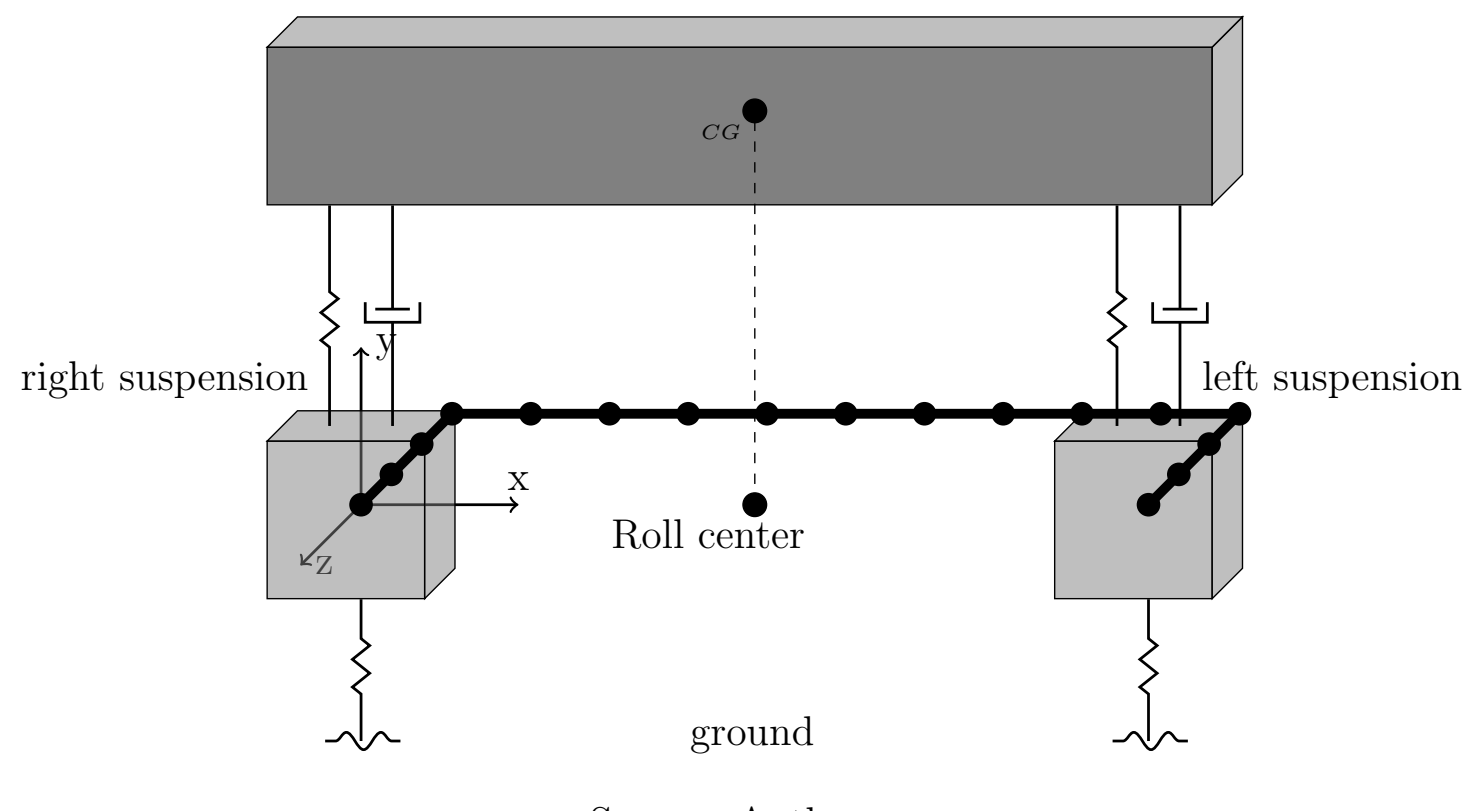

Source: Author.

The simplest roll dynamics model for a vehicle can consist of just one d.o.f. motion, as shown in Figure 8. To begin with, assume that the axles and tires have negligible mass compared to the vehicle chassis. Also, assume that the vehicle is on a flat road, i.e. with no inclination. The single d.o.f. considered is the roll angle $\phi$, as shown in Figure 8 (RAJAMANI, 2012). 
Figure 8: Roll dynamics and free body diagram.

(a) Schematic for vehicle roll dynamics for 1 d.o.f. model.

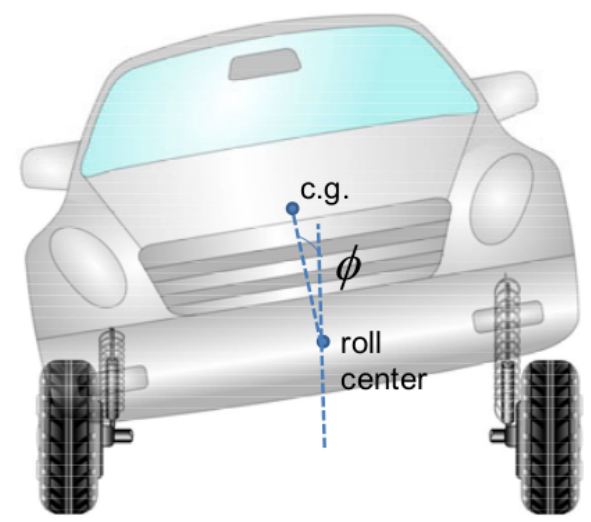

(b) Free body diagram for 1 d.o.f. roll model.

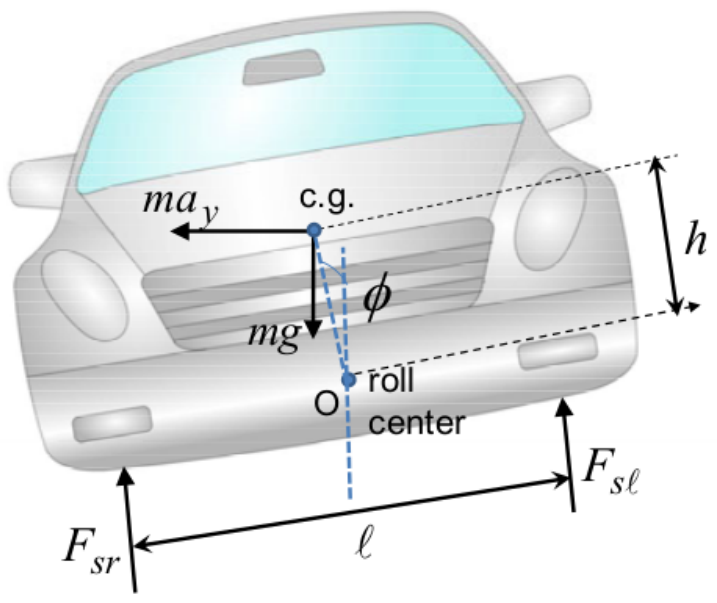

Source: (RAJAMANI, 2012).

Assume that the total vehicle mass is $m$, the roll moment of inertia about the c.g. is $I_{x x}$, the distance between left and right suspensions locations is $l$, and the height of the c.g. of the sprung mass from the roll center is $h$. Let $a_{y}$ be the lateral acceleration experienced by the vehicle. Let the left and right dynamics suspension forces be denoted by $F_{s l}$ and $F_{r l}$, respectively. From the lateral dynamics of the vehicle, one has $F_{l a t}=m a_{y}$. It should be noted that the d'Alembert's principle has been applied at the c.g. of the vehicle in order to make it a quasi-static system, i.e. the process happens slowly enough for the system to remain in internal equilibrium (RAJAMANI, 2012).

Taking moments about the roll center $\mathrm{O}$, the roll dynamics equation can be written 
as:

$$
\begin{aligned}
& \left(I_{x x}+m h^{2}\right) \ddot{\phi}=\sum M_{x} \\
& =F_{l a t} h \cos \phi+m g h \sin \phi+F_{s l} \frac{l}{2}-F_{s r} \frac{l}{2},
\end{aligned}
$$

where the total moment of inertia $\left(I_{x x}+m h^{2}\right)$ is obtained by axles parallel theorem. Then:

$$
\left(I_{x x}+m h^{2}\right) \ddot{\phi}=F_{l a t} h \cos \phi+m g h \sin \phi+\frac{l}{2}\left(F_{s l}-F_{s r}\right) .
$$

The suspension forces $F_{s l}$ and $F_{s r}$ act on both sides of each of the suspension spring, as shown in Figure 9. The sprung mass deflection on the right side due to roll is as follows:

$$
z_{s r}=-\frac{l}{2} \sin \phi
$$

and in the left side:

$$
z_{s l}=\frac{l}{2} \sin \phi
$$

Hence, the dynamic suspension forces are:

$$
\begin{gathered}
F_{s r}=k_{s} \frac{l}{2} \sin \phi+b_{s} \frac{l}{2} \dot{\phi} \cos \phi, \\
F_{s l}=-k_{s} \frac{l}{2} \sin \phi-b_{s} \frac{l}{2} \dot{\phi} \cos \phi .
\end{gathered}
$$

Figure 9: Suspension forces.
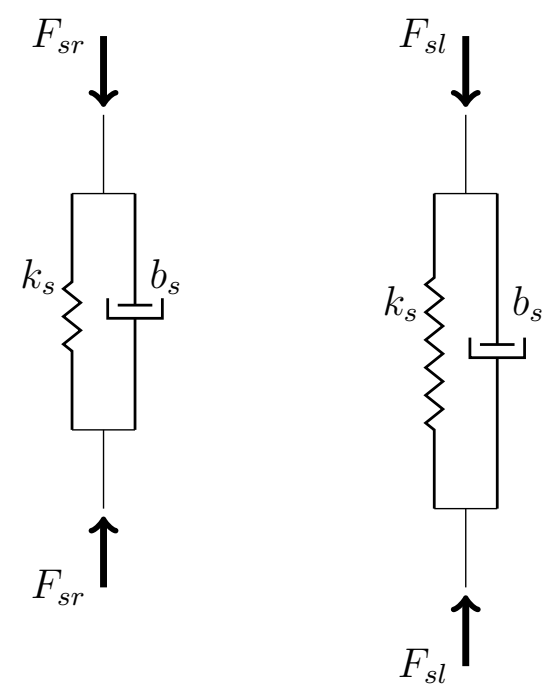

Source: Author. 
Substituting from Equation 3.5 and 3.6 into Equation 3.2 .

$$
\left(I_{x x}+m h^{2}\right) \ddot{\phi}=m a_{y} h \cos \phi+m g h \sin \phi-\frac{1}{2} k_{s} l^{2} \sin \phi-\frac{1}{2} b_{s} l^{2} \dot{\phi} \cos \phi .
$$

It should be noted that the roll dynamics depend on the lateral dynamics through the lateral acceleration term $a_{y}$. By avoiding further expansion of this term of lateral tire forces and lateral dynamic states, a complicated coupled set of equations between roll and lateral dynamics is avoided. Instead, the variable $a_{y}$ is assumed to be measured and is assumed to be a known input.

The static tire forces on the ground, in the absence of roll angle, are $m g / 2$ on the left tires and on the right tires. Including both dynamic and static forces, the total tire forces on the ground are, in the right side:

$$
F_{z r}=\frac{m g}{2}+F_{s r}=\frac{m g}{2}+k_{s} \frac{l}{2} \sin \phi+b_{s} \frac{l}{2} \dot{\phi} \cos \phi
$$

and in the left side:

$$
F_{z l}=\frac{m g}{2}+F_{s l}=\frac{m g}{2}-k_{s} \frac{l}{2} \sin \phi-b_{s} \frac{l}{2} \dot{\phi} \cos \phi
$$

\subsection{Four d.o.f. roll dynamics model}

The four d.o.f. considered in this model are:

- vertical translation of the sprung mass $m_{s}$, which is the car's heave, and denoted by $z_{s}$.

- roll motion of the sprung mass $m_{s}$, denoted by $\phi$.

- vertical translation of the right unsprung mass $m_{u r}$, which is the weight of the entire right wheel assembly, and denoted by $z_{u r}$.

- vertical translation of the left unsprung mass $m_{u l}$, which is the weight of the entire left wheel assembly, and denoted by $z_{u l}$.

The four d.o.f. $z_{s}, \phi, z_{u r}$ and $z_{u l}$ are shown in Figure 10. The variables $z_{r r}$ e $z_{r l}$ are roads inputs that act on the system. Inclusion of the influence of the road profiles implies that roll motion induced by road bumps and potholes can also be analyzed by this model in addition to roll motion induced by lateral acceleration. 
Assume in this work that the motions $z_{s}, z_{u r}$ e $z_{u l}$ are measured from the position of static equilibrium. Let the dynamic suspension forces on the left and right sides be denoted by $F_{s r}$ and $F_{s l}$ respectively and the dynamic tire forces on the left and right tires be denoted by $F_{t r}$ e $F_{t l}$ respectively, as shown in Figure 10.

Figure 10: Four d.o.f. roll dynamics model.

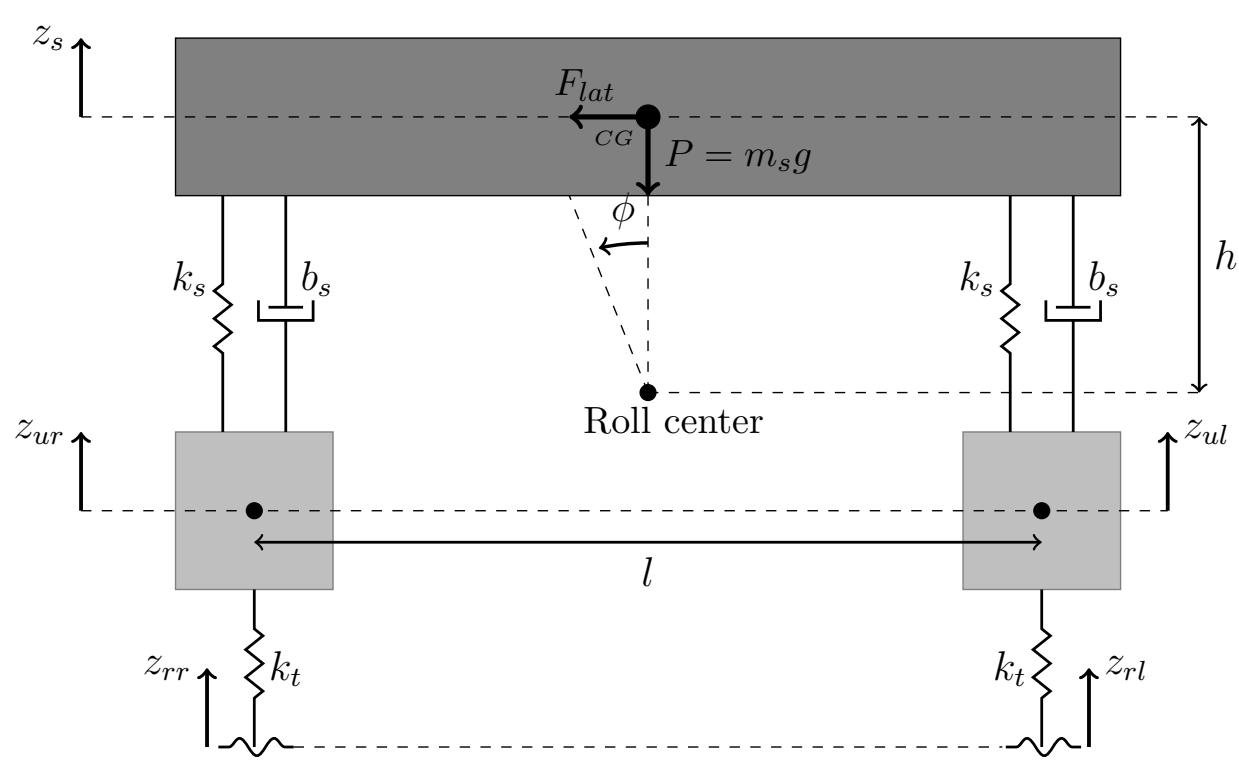

Source: Author.

Vertical force balance on the sprung mass and the two unsprung masses yield the following equations:

$$
\begin{gathered}
m_{s} \ddot{z}_{s}=F_{s r}+F_{s l}, \\
m_{u r} \ddot{z}_{u r}=-F_{s r}+F_{t r}, \\
m_{u l} \ddot{z}_{u l}=-F_{s l}+F_{t l} .
\end{gathered}
$$

The roll dynamics of the sprung mass are given by the following equation as developed in the previous section:

$$
J_{s} \ddot{\phi}=m_{s} a_{y} h \cos \phi+m_{s} g h \sin \phi+\frac{l}{2}\left(F_{s l}-F_{s r}\right),
$$

where $J_{s}=I_{x x}+m_{s} h^{2}$.

The dynamic suspension forces are given by:

$$
\begin{aligned}
F_{s r} & =-k_{s}\left(z_{s r}-z_{u r}\right)-b_{s}\left(\dot{z}_{s r}-\dot{z}_{u r}\right) \\
& =-k_{s}\left(z_{s}-\frac{l}{2} \sin \phi-z_{u r}\right)-b_{s}\left(\dot{z}_{s}-\frac{l}{2} \dot{\phi} \cos \phi-\dot{z}_{u r}\right)
\end{aligned}
$$




$$
\begin{aligned}
F_{s l} & =-k_{s}\left(z_{s l}-z_{u l}\right)-b_{s}\left(\dot{z}_{s l}-\dot{z}_{u l}\right) \\
& =-k_{s}\left(z_{s}+\frac{l}{2} \sin \phi-z_{u l}\right)-b_{s}\left(\dot{z}_{s}+\frac{l}{2} \dot{\phi} \cos \phi-\dot{z}_{u l}\right),
\end{aligned}
$$

and the dynamic tire forces are given by:

$$
\begin{gathered}
F_{t r}=-k_{t}\left(z_{u r}-z_{r r}\right), \\
F_{t l}=-k_{t}\left(z_{u l}-z_{r l}\right) .
\end{gathered}
$$

From Equation 3.14 and 3.15 .

$$
F_{s l}-F_{s r}=-k_{s} l \sin \phi-b_{s} l \dot{\phi} \cos \phi+k_{s}\left(z_{u l}-z_{u r}\right)+b_{s}\left(\dot{z}_{u l}-\dot{z}_{u r}\right)
$$

Substituting Equation 3.18 into Equation 3.13 .

$$
\begin{aligned}
J_{s} \ddot{\phi}= & m_{s} a_{y} h \cos \phi+m_{s} g h \sin \phi-\frac{k_{s} l^{2}}{2} \sin \phi-\frac{b_{s} l^{2}}{2} \dot{\phi} \cos \phi \\
& +\frac{k_{s} l}{2}\left(z_{u l}-z_{u r}\right)+\frac{b_{s} l}{2}\left(\dot{z}_{u l}-\dot{z}_{u r}\right) .
\end{aligned}
$$

Hence, the overall equations of motion for the model is given by:

$$
\begin{gathered}
m_{s} \ddot{z}_{s}=-k_{s}\left(2 z_{s}-z_{u r}-z_{u l}\right)-b_{s}\left(2 \dot{z}_{s}-\dot{z}_{u r}-\dot{z}_{u l}\right), \\
m_{u r} \ddot{z}_{u r}=k_{s}\left(z_{s}-\frac{l}{2} \sin \phi-z_{u r}\right)+b_{s}\left(\dot{z}_{s}-\frac{l}{2} \dot{\phi} \cos \phi-\dot{z}_{u r}\right)-k_{t}\left(z_{u r}-z_{r r}\right), \\
m_{u l} \ddot{z}_{u l}=k_{s}\left(z_{s}+\frac{l}{2} \sin \phi-z_{u l}\right)+b_{s}\left(\dot{z}_{s}+\frac{l}{2} \dot{\phi} \cos \phi-\dot{z}_{u l}\right)-k_{t}\left(z_{u l}-z_{r l}\right),
\end{gathered}
$$

and

$$
\begin{aligned}
J_{s} \ddot{\phi}= & m_{s} a_{y} h \cos \phi+m_{s} g h \sin \phi-\frac{k_{s} l^{2}}{2} \sin \phi-\frac{b_{s} l^{2}}{2} \dot{\phi} \cos \phi \\
& +\frac{k_{s} l}{2}\left(z_{u l}-z_{u r}\right)+\frac{b_{s} l}{2}\left(\dot{z}_{u l}-\dot{z}_{u r}\right),
\end{aligned}
$$

where $J_{s}=\left(I_{x x}+m_{s} h^{2}\right)$.

The total tire forces on the ground include both static force due to weight and dynamic forces. Including both static and dynamic forces, the total tire forces on the ground are given by:

$$
\begin{aligned}
& F_{z r}=F_{t r}+\frac{m_{s}}{2} g+m_{u r} g \\
& F_{z l}=F_{t l}+\frac{m_{s}}{2} g+m_{u l} g .
\end{aligned}
$$




\subsubsection{State-space model}

Aiming to make easy the analysis of the governing equations, the state-space representation is applied regarding the following states:

$x_{1}: \phi$, roll angle

$x_{2}: \dot{\phi}$, rate of roll angle

$x_{3}: z_{s}$, vertical translation of the sprung mass

$x_{4}: \dot{z}_{s}$, rate of vertical translation of the sprung mass

$x_{5}: z_{u r}$, vertical translation of the right unsprung mass

$x_{6}: \dot{z}_{u r}$, rate of vertical translation of the right unsprung mass

$x_{7}: z_{u l}$, vertical translation of the left unsprung mass

$x_{8}: \dot{z}_{u l}$, rate of vertical translation of the left unsprung mass

Considering roll angles smaller than 5 degrees, is suitable for the equations that $\cos \phi \approx 1$ and $\sin \phi \approx \phi$. Hence, the state equations become linear, and:

$$
\begin{aligned}
& \dot{x}_{1}=\dot{\phi}=x_{2}, \\
& \dot{x}_{2}=\ddot{\phi}=\frac{1}{J_{s}}\left[m_{s} h a_{y}+\left(m_{s} g h-\frac{k_{s} l^{2}}{2}\right) x_{1}-\frac{b_{s} l^{2}}{2} x_{2}+\frac{k_{s} l}{2}\left(x_{7}-x_{5}\right)+\frac{b_{s} l}{2}\left(x_{8}-x_{6}\right)\right], \\
& \dot{x}_{3}=\dot{z}_{s}=x_{4}, \\
& \dot{x}_{4}=\ddot{z}_{s}=\frac{-1}{m_{s}}\left[k_{s}\left(2 x_{3}-x_{5}-x_{7}\right)+b_{s}\left(2 x_{4}-x_{6}-x_{8}\right)\right], \\
& \dot{x}_{5}=\dot{z}_{u r}=x_{6}, \\
& \dot{x}_{6}=\ddot{z}_{u r}=\frac{-1}{m_{u r}}\left[k_{s}\left(\frac{l}{2} x_{1}-x_{3}+x_{5}\right)+b_{s}\left(\frac{l}{2} x_{2}-x_{4}+x_{6}\right)-k_{t}\left(x_{5}-z_{r r}\right)\right], \\
& \dot{x}_{7}=\dot{z}_{u l}=x_{8}, \\
& \dot{x}_{8}=\ddot{z}_{u l}=\frac{1}{m_{u l}}\left[k_{s}\left(\frac{l}{2} x_{1}+x_{3}+x_{7}\right)+b_{s}\left(\frac{l}{2} x_{2}+x_{4}-x_{8}\right)-k_{t}\left(x_{7}-z_{r l}\right)\right] .
\end{aligned}
$$

Therefore, the lateral acceleration $a_{y}$ and the road profiles $z_{r r}$ e $z_{r l}$ are the model inputs, which will be treated as system disturbances. The state-space matricial equation is:

$$
\begin{aligned}
& \dot{x}=A x+B u, \\
& y=C x,
\end{aligned}
$$


where:

$$
\begin{aligned}
& A=\left[\begin{array}{cccccccc}
0 & 1 & 0 & 0 & 0 & 0 & 0 & 0 \\
\frac{2 m_{s} g h-k_{s} l}{2 J_{s}} & -\frac{b_{s} l^{2}}{2 J_{s}} & 0 & 0 & -\frac{k_{s} l}{2 J_{s}} & -\frac{b_{s} l}{2 J_{s}} & \frac{k_{s} l}{2 J_{s}} & \frac{b_{s} l}{2 J_{s}} \\
0 & 0 & 0 & 1 & 0 & 0 & 0 & 0 \\
0 & 0 & -\frac{2 k_{s}}{m_{s}} & -\frac{2 b_{s}}{m_{s}} & \frac{k_{s}}{m_{s}} & \frac{b_{s}}{m_{s}} & \frac{k_{s}}{m_{s}} & \frac{b_{s}}{m_{s}} \\
0 & 0 & 0 & 0 & 0 & 1 & 0 & 0 \\
-\frac{k_{s} l}{2 m_{u r}} & -\frac{b_{s} l}{2 m_{u r}} & \frac{k_{s}}{m_{u r}} & \frac{b_{s}}{m_{u r}} & -\frac{k_{s}+k_{t}}{m_{u r}} & -\frac{b_{s}}{m_{u r}} & 0 & 0 \\
0 & 0 & 0 & 0 & 0 & 0 & 0 & 1 \\
\frac{k_{s} l}{2 m_{u l}} & \frac{b_{s} l}{2 m_{u l}} & \frac{k_{s}}{m_{u l}} & \frac{b_{s}}{m_{u l}} & 0 & 0 & -\frac{k_{s}+k_{t}}{m_{u l}} & -\frac{b_{s}}{m_{u l}}
\end{array}\right] \\
& B=\left[\begin{array}{cccccccc}
0 & \frac{m_{s} h}{J_{s}} & 0 & 0 & 0 & 0 & 0 & 0 \\
0 & 0 & 0 & 0 & 0 & k_{t} & 0 & 0 \\
0 & 0 & 0 & 0 & 0 & 0 & 0 & k_{t}
\end{array}\right]^{T} \\
& C=\left[\begin{array}{llllllll}
1 & 0 & 0 & 0 & 0 & 0 & 0 & 0
\end{array}\right] \text {, }
\end{aligned}
$$

where for the output matrix $C$ is considered the measure of the roll angle by a sensor and $u=\left[\begin{array}{lll}a_{y} & z_{r r} & z_{r l}\end{array}\right]^{T}$.

\subsection{Inclusion of the passive anti-roll bar}

In this section, the passive anti-roll bar will be included to the model of the previous section. The anti-roll bar is illustrated in Figure 11 and it will be divided in elements later. The generalized Hamilton's principle is given by:

$$
\int_{t_{1}}^{t_{2}}\left(\delta(T-V)+\delta W_{n c}\right) \mathrm{d} t=0
$$

where $T$ is the total kinetic energy of the system, $V$ is the potential energy of the system (including strain energy and the potential energy of conservative external forces), $\delta()$ represents the virtual displacement (or variational) operator, and $\delta W_{n c}$ is the virtual work done by non-conservative forces (including damping forces and external forces not accounted for in $V$ ). 
Figure 11: Roll dynamics model with passive anti-roll bar.

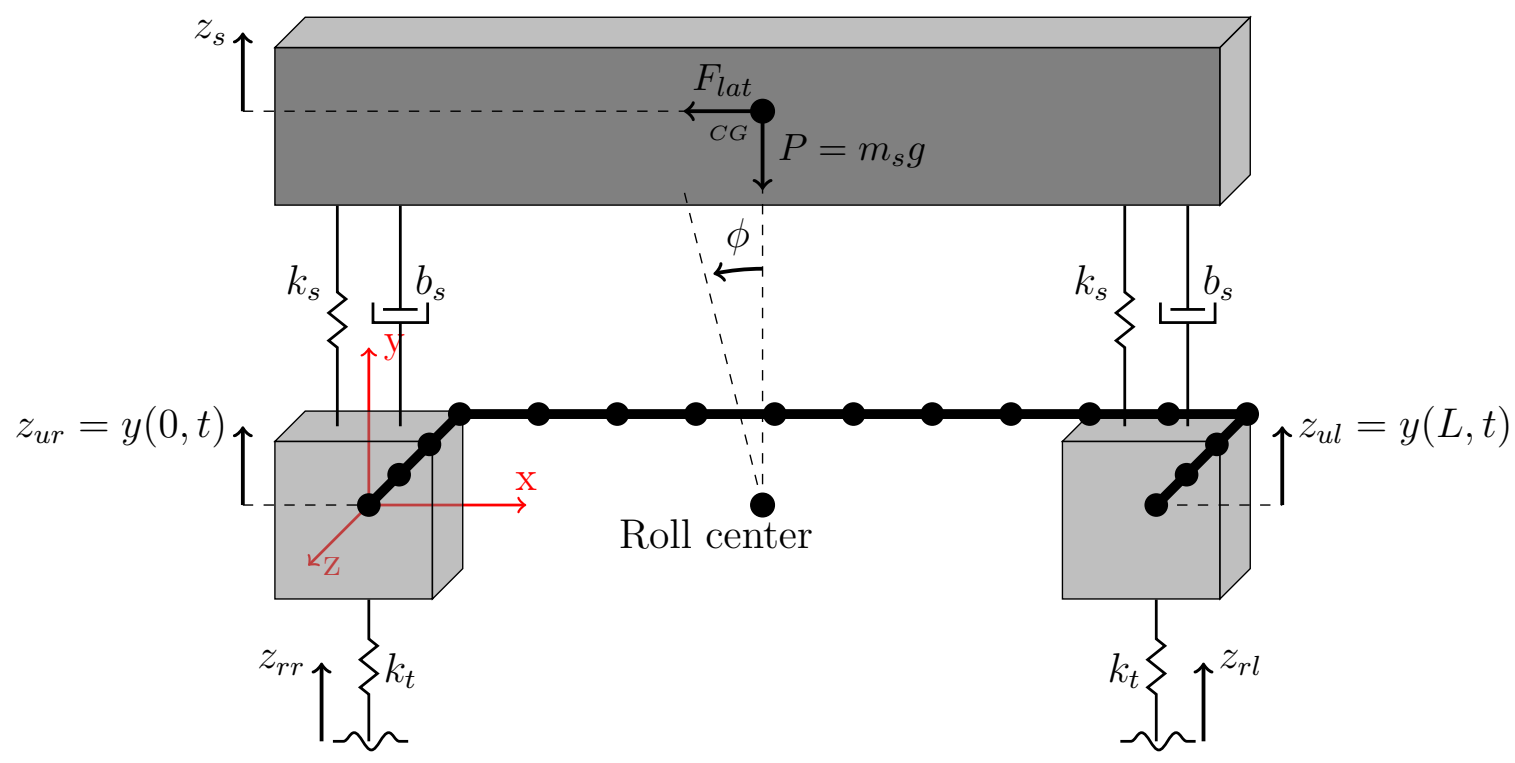

Source: Author.

Considering the Lagrangian defined in the classical form $\mathcal{L}=T-V$, it is assumed that the Lagrangian consists of three terms such as:

$$
\mathcal{L}=L_{D}+\int_{\Omega} \hat{L} \mathrm{~d} \Omega+L_{B}
$$

where $\Omega$ is the spacial domain occupied by the undeformed flexible body, $L_{D}$ is the discrete portion of $\mathcal{L}, \hat{L}$ is the Lagrangian density and $L_{B}$ is the boundary term portion of the Lagrangian energy functional, which in turn depends on the boundary motions (JUNKINS; KIM, 1993). This division is called hybridization between discrete and distributed variables and boundary conditions. The kinetic and potential energy of the system in Figure 11 are as follows:

$$
T=\frac{m_{s} \dot{z}_{s}^{2}}{2}+\frac{m_{u r} \dot{y}^{2}(0, t)}{2}+\frac{m_{u l} \dot{y}^{2}(L, t)}{2}+\frac{J_{s} \dot{\phi}^{2}}{2}+\frac{1}{2} \int_{0}^{L} \rho \dot{y}^{2}(x, t) \mathrm{d} x,
$$

where $J_{s}=\left(I_{x x}+m_{s} h^{2}\right) / 2$.

$$
\begin{aligned}
& V=m_{s} g h \cos (\phi)+\frac{k_{t}}{2}\left(y(0, t)-z_{r r}\right)^{2}+\frac{k_{t}}{2}\left(y(L, t)-z_{r l}\right)^{2} \\
& +\frac{k_{s}}{2}\left(z_{s}-\frac{l}{2} \sin (\phi)-y(0, t)\right)^{2}+\frac{k_{s}}{2}\left(z_{s}+\frac{l}{2} \sin (\phi)-y(L, t)\right)^{2} \\
& +\frac{1}{2} \int_{0}^{L} E I\left(y^{\prime \prime}(x, t)\right)^{2} \mathrm{~d} x
\end{aligned}
$$


Hence, the Lagrangian can be divided as:

$$
\begin{aligned}
& L_{D}=T_{D}-V_{D}, \\
& \hat{L}=\hat{T}-\hat{V}, \\
& L_{B}=T_{B}-V_{B} .
\end{aligned}
$$

Separating the discrete, distributed and boundary terms of the Equation 3.31 and Equation 3.32 one has:

- Discrete (or lumped) term:

$$
\begin{aligned}
& L_{D}=\frac{J_{s} \dot{\phi}^{2}}{2}+\frac{m_{s} \dot{z}_{s}^{2}}{2}-m_{s} g h \cos (\phi)-k_{s} z_{s}^{2}-k_{s} \frac{l^{2}}{4} \sin ^{2}(\phi), \\
& L_{D}=L_{D}\left(\phi, \dot{\phi}, z_{s}, \dot{z}_{s}\right),
\end{aligned}
$$

- Distributed term:

$$
\begin{aligned}
\hat{L} & =\frac{1}{2} \rho \dot{y}^{2}(x, t)-\frac{1}{2} E I\left(y^{\prime \prime}(x, t)\right)^{2}, \\
\hat{L} & =\hat{L}\left(y^{\prime \prime}(x, t), \dot{y}(x, t)\right),
\end{aligned}
$$

- Boundary term:

$$
\begin{aligned}
& L_{B}=\frac{m_{u r} \dot{y}^{2}(0, t)}{2}+\frac{m_{u l} \dot{y}^{2}(L, t)}{2}-\frac{k_{t}}{2}\left(y(0, t)-z_{r r}\right)^{2}-\frac{k_{t}}{2}\left(y(L, t)-z_{r l}\right)^{2} \\
& -\frac{k_{s} y^{2}(0, t)}{2}-\frac{k_{s} y^{2}(L, t)}{2}-\frac{k_{s}}{2} l \sin (\phi)(y(0, t)-y(L, t))+k_{s} z_{s}(y(0, t)+y(L, t)), \\
& L_{B}=L_{B}\left(y(0, t), y(L, t), \dot{y}(0, t), \dot{y}(L, t), \phi, z_{s}, \dot{z}_{s}\right) .
\end{aligned}
$$

\subsubsection{Non-conservative work $W_{n c}$}

The lateral force denoted by $F_{\text {lat }}$, is an external input, and, then, treated as external energy incoming to the system, which characterizes a non-conservative work. It is described by:

$$
W_{F_{l a t}}=F_{l a t} h \sin \phi=W_{F_{l a t}}(\phi) .
$$

The dampers are responsible to dissipate the energy stored in the springs by friction 
dissipation. It is also a non-conservative energy given by:

$$
\begin{aligned}
& W_{\text {fric }}=\frac{1}{2} b_{s}\left(\dot{z}_{s}-\frac{l}{2} \dot{\phi} \cos \phi-\dot{y}(0, t)\right)^{2}+\frac{1}{2} b_{s}\left(\dot{z}_{s}+\frac{l}{2} \dot{\phi} \cos \phi-\dot{y}(L, t)\right)^{2}, \\
& W_{\text {fric }}=W_{\text {fric }}\left(\phi, \dot{\phi}, \dot{z}_{s}, \dot{y}(0, t), \dot{y}(L, t)\right) .
\end{aligned}
$$

Hence, the total non-conservative work is given as $W_{n c}=W_{F_{l a t}}+W_{\text {fric }}$.

\subsubsection{Equations of motion}

In order to use the above equations into Equation 3.29, the calculation of variations are needed. In the following, the variations are obtained for each equation. The virtual work terms are:

$$
\begin{gathered}
\delta W_{F_{\text {lat }}}=\frac{\partial W_{F_{\text {lat }}}}{\partial \phi} \delta \phi \\
\delta W_{\text {fric }}=\frac{\partial W_{\text {fric }}}{\partial \phi} \delta \phi+\frac{\partial W_{\text {fric }}}{\partial \dot{\phi}} \delta \dot{\phi}+\frac{\partial W_{\text {fric }}}{\partial \dot{z}_{s}} \delta \dot{z}_{s}+\frac{\partial W_{\text {fric }}}{\partial \dot{y}(0, t)} \delta \dot{y}(0, t)+\frac{\partial W_{\text {fric }}}{\partial \dot{y}(L, t)} \delta \dot{y}(L, t), \\
\left.\delta W_{n c}=\left[F_{\text {lat }} h \cos \phi+b_{s} \frac{l}{2} \cos \phi(\dot{y}(L, t)-\dot{y}(0, t))-b_{s} \frac{l^{2}}{2} \dot{\phi} \cos ^{2} \phi\right)\right] \delta \phi \\
+\left[b_{s}(\dot{y}(0, t)+\dot{y}(L, t))-2 b_{s} \dot{z}_{s}\right] \delta z_{s}+\left[b_{s}\left(\dot{z}_{s}-\frac{l}{2} \dot{\phi} \cos \phi-\dot{y}(0, t)\right)\right] \delta y(0, t) \\
+\left[b_{s}\left(\dot{z}_{s}+\frac{l}{2} \dot{\phi} \cos \phi-\dot{y}(L, t)\right)\right] \delta y(L, t) .
\end{gathered}
$$

and the variations of the discrete, distributed and boundary terms are, respectively:

$$
\begin{gathered}
\delta L_{D}=\frac{\partial L_{D}}{\partial \phi} \delta \phi+\frac{\partial L_{D}}{\partial \dot{\phi}} \delta \dot{\phi}+\frac{\partial L_{D}}{\partial z_{s}} \delta z_{s}+\frac{\partial L_{D}}{\partial \dot{z}_{s}} \delta \dot{z}_{s}, \\
\delta L_{D}=\left[m_{s} g h \sin \phi-k_{s} \frac{l^{2}}{2} \sin \phi \cos \phi\right] \delta \phi+J_{s} \dot{\phi} \delta \dot{\phi}-2 k_{s} z_{s} \delta z_{s}+m_{s} \dot{z}_{s} \delta \dot{z}_{s}, \\
\delta \hat{L}=\frac{\partial \hat{L}}{\partial y^{\prime \prime}(x, t)} \delta y^{\prime \prime}(x, t)+\frac{\partial \hat{L}}{\partial \dot{y}(x, t)} \delta \dot{y}(x, t)=-E I y^{\prime \prime}(x, t) \delta y^{\prime \prime}(x, t)+\rho \dot{y}(x, t) \delta \dot{y}(x, t),
\end{gathered}
$$




$$
\begin{aligned}
& \delta L_{B}=\frac{\partial L_{B}}{\partial y(0, t)} \delta y(0, t)+\frac{\partial L_{B}}{\partial y(L, t)} \delta y(L, t)+\frac{\partial L_{B}}{\partial \dot{y}(0, t)} \delta \dot{y}(0, t) \\
& +\frac{\partial L_{B}}{\partial \dot{y}(L, t)} \delta \dot{y}(L, t)+\frac{\partial L_{B}}{\partial \phi} \delta \phi+\frac{\partial L_{B}}{\partial z_{s}} \delta z_{s}, \\
& \delta L_{B}=\left[k_{s} z_{s}-k_{s} \frac{l}{2} \sin \phi-k_{s} y(0, t)-k_{t}\left(y(0, t)-z_{r r}\right)\right] \delta y(0, t) \\
& +\left[k_{s} z_{s}+k_{s} \frac{l}{2} \sin \phi-k_{s} y(L, t)-k_{t}\left(y(L, t)-z_{r l}\right)\right] \delta y(L, t) \\
& +m_{u r} \dot{y}(0, t) \delta \dot{y}(0, t)+m_{u l} \dot{y}(L, t) \delta \dot{y}(L, t) \\
& -k_{s} \frac{l}{2} \cos \phi(y(0, t)-y(L, t)) \delta \phi+k_{s}[y(0, t)+y(L, t)] \delta z_{s} .
\end{aligned}
$$

The generalized Hamilton's principle is::

$$
\int_{t_{1}}^{t_{2}}\left(\delta L_{D}+\int_{\Omega} \delta \hat{L} \mathrm{~d} \Omega+\delta L_{B}+\delta W_{n c}\right) \mathrm{d} t=0
$$

where the four terms are:

- First term: $\int_{t_{1}}^{t_{2}} \delta L_{D} \mathrm{~d} t$

$$
\int_{t_{1}}^{t_{2}}\left\{\left[m_{s} g h \sin \phi-k_{s} \frac{l^{2}}{2} \sin \phi \cos \phi\right] \delta \phi+J_{s} \dot{\phi} \delta \dot{\phi}-2 k_{s} z_{s} \delta z_{s}+m_{s} \dot{z}_{s} \delta \dot{z}_{s}\right\} \mathrm{d} t
$$

and integrating Equation 3.46 by parts and some manipulations give:

$$
\int_{t_{1}}^{t_{2}}\left\{\left[m_{s} g h \sin \phi-k_{s} \frac{l^{2}}{2} \sin \phi \cos \phi-J_{s} \ddot{\phi}\right] \delta \phi-\left[2 k_{s} z_{s}+m_{s} \ddot{z}_{s}\right] \delta z_{s}\right\} \mathrm{d} t .
$$

- Second term: $\int_{t_{1}}^{t_{2}} \int_{\Omega} \delta \hat{L} \mathrm{~d} \Omega \mathrm{d} t$

$$
\int_{t_{1}}^{t_{2}} \int_{0}^{L}\left[\rho \dot{y}(x, t) \delta \dot{y}(x, t)-E I y^{\prime \prime}(x, t) \delta y^{\prime \prime}(x, t),\right] \mathrm{d} \Omega \mathrm{d} t
$$

and integrating Equation 3.48 by parts and some manipulations give:

$$
\begin{aligned}
& \int_{t_{1}}^{t_{2}}\left\{\int_{0}^{L}\left[-\rho \ddot{y}(x, t)-E I y^{\prime \prime \prime \prime}(x, t)\right] \delta y(x, t) \mathrm{d} x\right. \\
& \left.-\left.E I y^{\prime \prime}(x, t) \delta y^{\prime}(x, t)\right|_{x=L} ^{x=0}+\left.E I y^{\prime \prime \prime}(x, t) \delta y(x, t)\right|_{x=L} ^{x=0}\right\} \mathrm{d} t .
\end{aligned}
$$


- Third term: $\int_{t_{1}}^{t_{2}} \delta L_{B} \mathrm{~d} t$

$$
\begin{aligned}
& \int_{t_{1}}^{t_{2}}\left\{\left[k_{s} z_{s}-k_{s} \frac{l}{2} \sin \phi-k_{s} y(0, t)-k_{t}\left(y(0, t)-z_{r r}\right)\right] \delta y(0, t)\right. \\
& +\left[k_{s} z_{s}+k_{s} \frac{l}{2} \sin \phi-k_{s} y(L, t)-k_{t}\left(y(L, t)-z_{r l}\right)\right] \delta y(L, t) \\
& +m_{u r} \dot{y}(0, t) \delta \dot{y}(0, t)+m_{u l} \dot{y}(L, t) \delta \dot{y}(L, t) \\
& \left.-k_{s} \frac{l}{2} \cos \phi[y(0, t)-y(L, t)] \delta \phi+k_{s}[y(0, t)+y(L, t)] \delta z_{s}\right\} \mathrm{d} t
\end{aligned}
$$

and integrating Equation 3.50 by parts and some manipulations give:

$$
\begin{aligned}
& \int_{t_{1}}^{t_{2}}\left\{\left[k_{s} z_{s}-k_{s} \frac{l}{2} \sin \phi-k_{s} y(0, t)-k_{t}\left(y(0, t)-z_{r r}\right)\right] \delta y(0, y)\right. \\
& +\left[k_{s} z_{s}+k_{s} \frac{l}{2} \sin \phi-k_{s} y(L, t)-k_{t}\left(y(L, t)-z_{r l}\right)\right] \delta y(L, y) \\
& \left.+\left[-k_{s} \frac{l}{2} \cos \phi(y(0, t)-y(L, t))\right] \delta \phi+\left[k_{s}(y(0, t)+y(L, t))\right] \delta z_{s}\right\} \mathrm{d} t .
\end{aligned}
$$

- Fourth term: $\int_{t_{1}}^{t_{2}} \delta W_{n c} \mathrm{~d} t$

$$
\begin{aligned}
& \int_{t_{1}}^{t_{2}}\left\{\left[F_{l a t} h \cos \phi-b_{s} \frac{l}{2} \cos \phi(\dot{y}(L, t)-\dot{y}(0, t)-l \dot{\phi} \cos \phi)\right] \delta \phi\right. \\
& +\left[b_{s}(\dot{y}(0, t)+\dot{y}(L, t))-2 b_{s} \dot{z}_{s}\right] \delta z_{s}+\left[b_{s}\left(\dot{z}_{s}-\frac{l}{2} \dot{\phi} \cos \phi-\dot{y}(0, t)\right)\right] \delta y(0, t) \\
& +\left[b_{s}\left(\dot{z}_{s}+\frac{l}{2} \dot{\phi} \cos \phi-\dot{y}(L, t)\right)\right] \delta y(L, t) .
\end{aligned}
$$

Gathering all terms for each variation yields to the generalized Hamilton's equation 
as follows:

$$
\begin{aligned}
& \int_{t_{1}}^{t_{2}}\left\{\left[m_{s} g h \sin \phi-k_{s} \frac{l^{2}}{2} \sin \phi \cos \phi-J_{s} \ddot{\phi}-k_{s} \frac{l}{2} \cos \phi(y(0, t)-y(L, t))\right.\right. \\
& \left.-b_{s} \frac{l}{2} \cos \phi(\dot{y}(L, t)-\dot{y}(0, t)-l \dot{\phi} \cos \phi)+F_{l a t} h \cos \phi\right] \delta \phi \\
& +\left[-m_{s} \ddot{z}_{s}-2 k_{s} z_{s}+k_{s}(y(0, t)+y(L, t))+b_{s}(\dot{y}(0, t)+\dot{y}(L, t))-2 b_{s} \dot{z}_{s}\right] \delta z_{s} \\
& -\int_{0}^{L}\left[\rho \ddot{y}(x, t)+E I y^{\prime \prime \prime \prime}(x, t)\right] \delta y(x, t) \mathrm{d} x-\left.E I y^{\prime \prime}(x, t) \delta y^{\prime}(x, t)\right|_{x=0} ^{x=L} \\
& +\left.E I y^{\prime \prime \prime}(x, t) \delta y(x, t)\right|_{x=0} ^{x=L}+\left[k_{s}\left(z_{s}+\frac{l}{2} \sin \phi-y(L, t)\right)-k_{t}\left(y(L, t)-z_{r l}\right)\right. \\
& \left.-m_{u l} \ddot{y}(L, t)+b_{s}\left(\dot{z}_{s}+\frac{l}{2} \dot{\phi} \cos \phi-\dot{y}(L, t)\right)\right] \delta y(L, t)+\left[k_{s}\left(z_{s}-\frac{l}{2} \sin \phi-y(0, t)\right)\right. \\
& \left.\left.-k_{t}\left(y(0, t)-z_{r r}\right)-m_{u r} \ddot{y}(L, t)+b_{s}\left(\dot{z}_{s}-\frac{l}{2} \dot{\phi} \cos \phi-\dot{y}(0, t)\right)\right] \delta y(0, t)\right\} \mathrm{d} t .
\end{aligned}
$$

Hence, the system's governing equations are:

1. Equation for roll dynamics:

$$
\begin{aligned}
& J_{s} \ddot{\phi}-m_{s} g h \sin \phi+k_{s} \frac{l^{2}}{2} \sin \phi \cos \phi+k_{s} \frac{l}{2} \cos \phi(y(0, t)-y(L, t)) \\
& +b_{s} \frac{l}{2} \cos \phi(\dot{y}(L, t)-\dot{y}(0, t)-l \dot{\phi} \cos \phi)-F_{l a t} h \cos \phi=0 .
\end{aligned}
$$

2. Equation for vertical translation dynamics of the sprung mass:

$$
m_{s} \ddot{z}_{s}+2 k_{s} z_{s}-k_{s}(y(0, t)+y(L, t))-b_{s}\left(\dot{y}(0, t)+\dot{y}(L, t)-2 \dot{z}_{s}\right)=0
$$

3. Equation for sway flexible bar:

$$
\rho \ddot{y}(x, t)+E I y^{\prime \prime \prime \prime}(x, t)=0 .
$$

The boundary conditions extracted from the generalized Hamilton's equation are:

1. Bending moment:

$$
\left.E I y^{\prime \prime}(x, t)\right|_{x=0} ^{x=L}=0
$$

2. Shear force: 
- To $x=0$ :

$$
\begin{aligned}
E I y^{\prime \prime \prime}(0, t)= & k_{s} z_{s}-k_{s} \frac{l}{2} \sin \phi-k_{s} y(0, t)-k_{t}\left(y(0, t)-z_{r r}\right) \\
& -m_{u r} \ddot{y}(0, t)+b_{s}\left(\dot{z}_{s}-\frac{l}{2} \dot{\phi} \cos \phi-\dot{y}(0, t)\right) .
\end{aligned}
$$

- To $x=L$ :

$$
\begin{aligned}
E I y^{\prime \prime \prime}(L, t)= & -k_{s} z_{s}-k_{s} \frac{l}{2} \sin \phi+k_{s} y(L, t)+k_{t}\left(y(L, t)-z_{r l}\right) \\
& +m_{u l} \ddot{y}(L, t)-b_{s}\left(\dot{z}_{s}+\frac{l}{2} \dot{\phi} \cos \phi-\dot{y}(L, t)\right) .
\end{aligned}
$$

\subsection{Search for solutions}

Now, it is necessary to search for solution for the global displacements $y(x, t)$, which are dependent of spatial coordinates and of time. Therefore, it deals with a set of partial differential equations. A spatial discretization technique needs to be applied to implement the solution numerically. According to Junkins and Kim, two spatial discretization methods can be used: the so-called assumed modes method, and the FEM. In this work, the FEM is chosen in order to model the anti-roll bar. For more information about the assumed modes method see (JUNKINS; KIM, 1993).

Let's define $y(x, t)$ to be a vector of displacements such that:

$$
y(x, t)=\left[\begin{array}{llllll}
v_{x}(x, t) & v_{y}(x, t) & v_{z}(x, t) & \theta_{x}(x, t) & \theta_{y}(x, t) & \theta_{z}(x, t)
\end{array}\right]^{T}
$$

where $v_{x}, v_{y}$ and $v_{z}$ are spatial displacements and $\theta_{x}, \theta_{y}$ and $\theta_{z}$ are spatial rotations in relation to axles $x, y$ and $z$, respectively. One applies the separation of variables, that is, one searches for solutions of the form:

$$
y(x, t)=\mathbf{N}(x) \mathbf{v}(t)
$$

where $\mathbf{v}(t)$ represents the nodal displacements and $\mathbf{N}(x)$ is a vector of shape functions, which must be selected to satisfy the conditions for admissibility. The acceleration and curvature in Equation 3.61 are expressed as follows:

$$
\begin{aligned}
\ddot{y}(x, t) & =\mathbf{N}(x) \ddot{\mathbf{v}}(t), \\
y^{\prime \prime}(x, t) & =\mathbf{N}^{\prime \prime}(x) \mathbf{v}(t) .
\end{aligned}
$$

In the following chapter, one searches for solutions in the form of Equation 3.61 based 
on the FEM concepts and on the nature of the problem which deals with a bar in the space of three dimensions.

\subsection{Hybridized model: FEM and discrete equations}

The candidate solution for displacements presented in Equation 3.61, is replaced into governing equations to establish the numerical model to be implemented. Substituting the Equation 3.61 and the Equation 3.62 into Equations 3.54 - 3.59, leads to:

1. Equation for roll dynamics:

$$
\begin{aligned}
& J_{s} \ddot{\phi}-m_{s} g h \sin \phi+k_{s} \frac{l^{2}}{2} \sin \phi+k_{s} \frac{l}{2} \cos \phi(\mathbf{N}(0) \mathbf{v}(t)-\mathbf{N}(L) \mathbf{v}(t)) \\
& +b_{s} \frac{l}{2} \cos \phi(\mathbf{N}(L) \dot{\mathbf{v}}(t)-\mathbf{N}(0) \dot{\mathbf{v}}(t)-l \dot{\phi} \cos \phi)-F_{l a t} h \cos \phi=0 .
\end{aligned}
$$

2. Equation for vertical translation dynamics of the sprung mass:

$$
\begin{aligned}
& m_{s} \ddot{z}_{s}+2 k_{s} z_{s}-k_{s}(\mathbf{N}(0) \mathbf{v}(t)+\mathbf{N}(L) \mathbf{v}(t)) \\
& -b_{s}\left(\mathbf{N}(0) \dot{\mathbf{v}}(t)+\mathbf{N}(L) \dot{\mathbf{v}}(t)-2 \dot{z}_{s}\right)=0
\end{aligned}
$$

3. Equation for the sway flexible bar:

$$
\rho \mathbf{N}(x) \ddot{\mathbf{v}}(t)+E I \mathbf{N}^{\prime \prime \prime \prime}(x) \mathbf{v}(t)=0,
$$

where the boundary conditions are:

1. Bending moment:

$$
\left.E I \mathbf{N}^{\prime \prime}(x) \mathbf{v}(t)\right|_{x=0} ^{x=L}=0
$$

2. Shear force:

- To $x=0$ :

$$
\begin{aligned}
& E I \mathbf{N}^{\prime \prime \prime}(0) \mathbf{v}(t)=k_{s} z_{s}-k_{s} \frac{l}{2} \sin \phi-k_{s} \mathbf{N}(0) \mathbf{v}(t)-k_{t}\left(\mathbf{N}(0) \mathbf{v}(t)-z_{r r}\right) \\
& -m_{u r} \mathbf{N}(0) \ddot{\mathbf{v}}(t)+b_{s}\left(\dot{z}_{s}-\frac{l}{2} \dot{\phi} \cos \phi-\mathbf{N}(0) \dot{\mathbf{v}}(t)\right)
\end{aligned}
$$


- To $x=L$ :

$$
\begin{aligned}
& E I \mathbf{N}^{\prime \prime \prime}(L) \mathbf{v}(t)=-k_{s} z_{s}-k_{s} \frac{l}{2} \sin \phi+k_{s} \mathbf{N}(L) \mathbf{v}(t)+k_{t}\left(\mathbf{N}(L) \mathbf{v}(t)-z_{r l}\right) \\
& +m_{u l} \mathbf{N}(L) \ddot{\mathbf{v}}(t)-b_{s}\left(\dot{z}_{s}+\frac{l}{2} \dot{\phi} \cos \phi-\mathbf{N}(L) \dot{\mathbf{v}}(t)\right)
\end{aligned}
$$

Over the distributed portion (Equation 3.65), the weighted residuals method is applied:

$$
\int_{0}^{L} \omega(x)\left[\rho \mathbf{N}(x) \ddot{\mathbf{v}}(t)+E I \mathbf{N}^{\prime \prime \prime \prime}(x) \mathbf{v}(t)\right] \mathrm{d} x=0,
$$

and integrating Equation 3.69 by parts over the time derivative terms and spatial derivative terms leads to:

$$
\begin{aligned}
& \int_{0}^{L} \omega(x) \rho \mathbf{N}(x) \mathrm{d} x \ddot{\mathbf{v}}(t)+\int_{0}^{L} \omega^{\prime \prime}(x) E I \mathbf{N}^{\prime \prime}(x) \mathrm{d} x \mathbf{v}(t) \\
& -\left.\omega^{\prime}(x) E I \mathbf{N}^{\prime \prime}(x)\right|_{x=0} ^{x=L} \mathbf{v}(t)+\left.\omega(x) E I \mathbf{N}^{\prime \prime \prime}(x)\right|_{x=0} ^{x=L} \mathbf{v}(t)=0 .
\end{aligned}
$$

By the Galerkin method, the choice of the weight function is:

$$
\omega(x)=\frac{\partial y(x, t)}{\partial v(t)}=\mathbf{N}^{T}(x)
$$

and the weighted residuals yields:

$$
M_{v} \ddot{\mathbf{v}}(t)+K_{v} \mathbf{v}(t)-\left.\mathbf{N}^{\prime T}(x) E I \mathbf{N}^{\prime \prime}(x)\right|_{x=0} ^{x=L} \mathbf{v}(t)+\left.\mathbf{N}^{T}(x) E I \mathbf{N}^{\prime \prime \prime}(x)\right|_{x=0} ^{x=L} \mathbf{v}(t)=0,
$$

where $M_{v}$ and $K_{v}$ are the mass and stiffness matrices provided from the finite element model, respectively. Its expressions are given by:

$$
M_{v}=\int_{0}^{L} \rho \mathbf{N}^{T}(x) \mathbf{N}(x) \mathrm{d} x, \quad K_{v}=\int_{0}^{L} E I \mathbf{N}^{\prime \prime T}(x) \mathbf{N}^{\prime \prime}(x) \mathrm{d} x .
$$

The boundary conditions obtained in Equations 3.66, 3.67, and 3.68 are introduced 
into Equation 3.72, yielding the complete hybridized model:

$$
\begin{aligned}
& M_{v} \ddot{\mathbf{v}}(t)+K_{v} \mathbf{v}(t)-\mathbf{N}^{\prime T}(L)\left[m_{u l} \mathbf{N}(L) \ddot{\mathbf{v}}(t)-b_{s}\left(\dot{z}_{s}+\frac{l}{2} \dot{\phi} \cos \phi-\mathbf{N}(L) \dot{\mathbf{v}}(t)\right)\right. \\
& \left.-k_{s} z_{s}-k_{s} \frac{l}{2} \sin \phi+k_{s} \mathbf{N}(L) \mathbf{v}(t)+k_{t}\left(\mathbf{N}(L) \mathbf{v}(t)-z_{r l}\right)\right] \\
& +\mathbf{N}^{\prime T}(0)\left[-m_{u r} \mathbf{N}(0) \ddot{\mathbf{v}}(t)+b_{s}\left(\dot{z}_{s}-\frac{l}{2} \dot{\phi} \cos \phi-\mathbf{N}(0) \dot{\mathbf{v}}(t)\right)\right. \\
& \left.+k_{s} z_{s}-k_{s} \frac{l}{2} \sin \phi-k_{s} \mathbf{N}(0) \mathbf{v}(t)-k_{t}\left(\mathbf{N}(0) \mathbf{v}(t)-z_{r r}\right)\right]=0 .
\end{aligned}
$$

Therefore, the completed model is formed by the Equations 3.63 , 3.64 and 3.74 . In the following chapter, will be developed the FEM model of the passive anti-roll bar. In other words, the vector of shaped functions $N(x)$ in Equation 3.61, will be built based on beam theory. 


\section{PASSIVE ANTI-ROLL BAR FEM MODEL}

In this chapter, one searches candidate solutions of the type $y(x, t)=\mathbf{N}(x) \mathbf{v}(t)$ for the completed model presented in the previous chapter. Therefore, the matrices $\mathbf{N}(x)$, $\mathbf{v}(t), M_{v}$ and $K_{v}$ presented in the previous chapter will be mounted for the whole bar, considering all elements. First, however, the mathematical model of one element will be deduced, followed by the coordinate transformations and assembly of the elements, in order to obtain the complete model for the bar.

\subsection{Beam element in three dimensions}

First of all, it will be searched candidate solutions for just one element, which will be described by the vector of displacements of the $i$ th element $y^{(i)}\left(x^{\prime}, t\right)=\mathbf{N}\left(x^{\prime}\right) \mathbf{v}(t)$, which is firstly expressed in the so-called local coordinate system $x^{\prime} y^{\prime} z^{\prime}$. From these local element solutions, the global displacements $y(x, t)$ will be obtained. Although the vector $y^{(i)}\left(x^{\prime}, t\right)$ is for the $i$ th element, it has the same structure as the global one in the Equation 3.60 .

Nodal d.o.f.'s are displacements and rotational components in a cartesian coordinate system which are the so-called primary variables we solve for. A three dimensional beam is the finite element used to model the anti-roll bar in this work and it has six d.o.f.'s in each node as shown in Figure 12, One element consists of:

- 2 nodes, one at each end (in this case), represented by $n_{1}$ and $n_{2}$;

- 3 deformation components [unit: $m$ ]: $v_{1}, v_{2}, v_{3}$ for $n_{1}$ and $v_{7}, v_{8}, v_{9}$ for $n_{2}$;

- 3 rotation components [unit: $\mathrm{rad}$ ]: $v_{4}, v_{5}, v_{6}$ for $n_{1}$ and $v_{10}, v_{11}, v_{12}$ for $n_{2}$; 
Figure 12: 3D beam element.

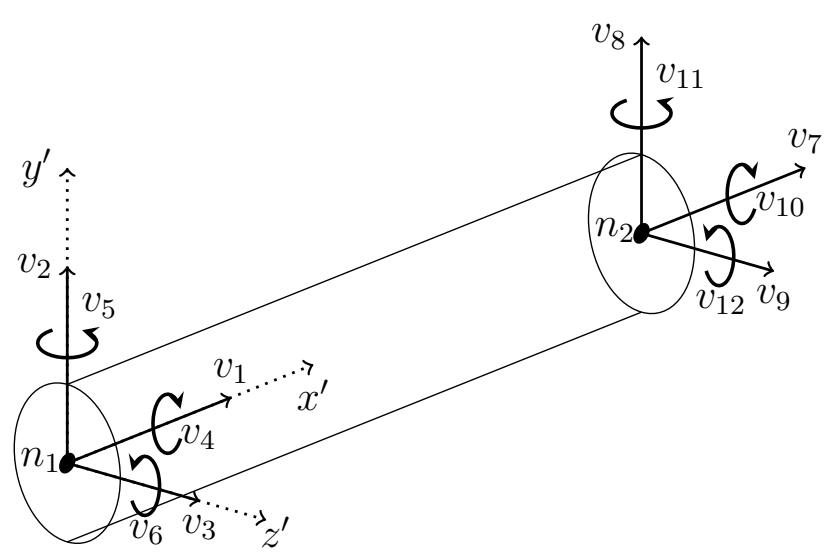

Source: Author.

Then, the total number of d.o.f. of an element is 12 . The assumptions taken to extract the equations of the $3 \mathrm{D}$ beam element are:

1. Small deformations. The axial deformation, bending and twisting can be decoupled and looked at separately.

2. For bending behavior, Bernoulli-Euler beam theory or Timoshenko beam theory may be used. In this work, the Bernoulli-Euler beam theory has been chosen for the calculation, which imply that the plane sections normal to the beam axis remain plane and normal to the beam axis during the deformation. For more information about the Timoshenko beam theory see (TIMOSHENKO; MACCULLOUGH et al., 1949).

3. Saint-Venant torsion theory for twisting is considered;

In the following, axial deformation, bending and twist are deduced according to the local coordinate system $x^{\prime} y^{\prime} z^{\prime}$ (Figure 12) and put into the all d.o.f. vector (Equation 3.60), building the discretized field to be used later.

\subsubsection{Axial deformation, $v_{x}^{(i)}$}

Consider for the axial deformation of an element, that will be written as $v_{x}^{(i)}$, the d.o.f.'s $v_{1}$ and $v_{7}$, as shown in Figure 13 . 
Figure 13: The d.o.f. for axial displacements.

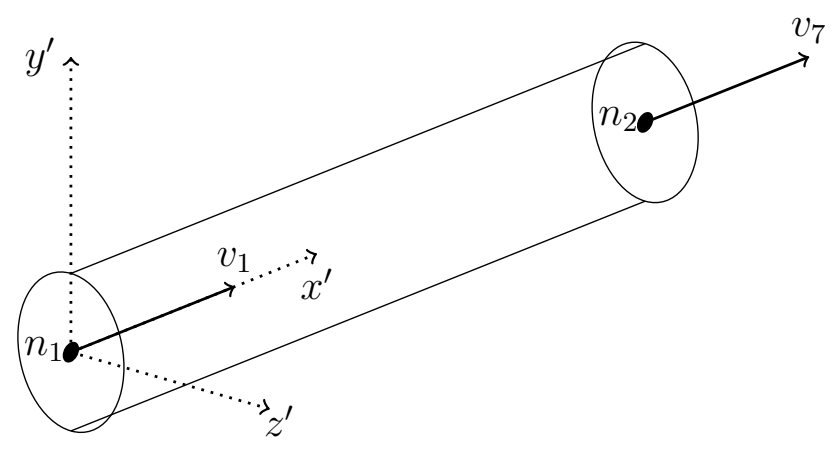

Source: Author.

Strong formulation. Let us define the following variables:

- $N\left(x^{\prime}\right)$ : normal force to the cross-section area $[N]$

- $A\left(x^{\prime}\right)$ : cross-section area $\left[m^{2}\right]$

- $b\left(x^{\prime}\right)$ : axially applied distributed force $[N]$

- $E\left(x^{\prime}\right)$ : Young's modulus $\left[N / m^{2}\right]$

- $\sigma$ : axial stress $\left[N / m^{2}\right]$

- $\epsilon$ : axial strain $[-]$

According to the Figure 14, the axial equilibrium equation of an infinitesimal portion is obtained by:

$$
\begin{aligned}
\sum F_{x} & =0 \\
-N+b d x^{\prime}+N+d N & =0 \\
\frac{d N}{d x^{\prime}}+b & =0 .
\end{aligned}
$$

Figure 14: Axial load diagram.
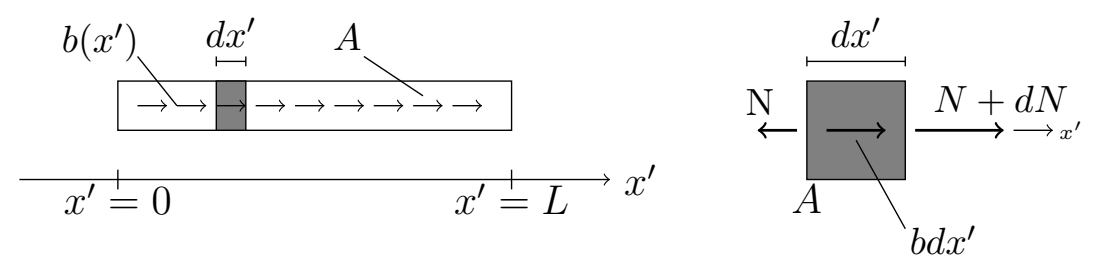

Source: Author. 
The force $N$ in terms of normal stress is given as:

$$
N=A\left(x^{\prime}\right) \sigma
$$

and by the constitutive relation, i.e. the material property by the Hooke's law:

$$
\sigma=E\left(x^{\prime}\right) \epsilon
$$

From a geometric relation, the strain can be obtained by the variation of the axial nodal displacement, that is:

$$
\epsilon=\frac{\partial v_{x}^{(i)}\left(x^{\prime}, t\right)}{\partial x^{\prime}}
$$

where $v_{x}^{(i)}\left(x^{\prime}, t\right)$ is the axial component of the general displacement $y^{(i)}\left(x^{\prime}, t\right)$, according to Equation 3.60. Hence, the axial deformation equation is governed by:

$$
\frac{\partial}{\partial x^{\prime}}\left(A\left(x^{\prime}\right) E\left(x^{\prime}\right) \frac{\partial v_{x}^{(i)}\left(x^{\prime}, t\right)}{\partial x^{\prime}}\right)+b=0, \quad 0 \leq x^{\prime} \leq L .
$$

Second-order differential equations, like Equation 4.5, needs two boundary conditions. The possible boundary conditions are displacements (kinematic) or displacement gradient (boundary force):

$$
\begin{aligned}
& v_{x}^{(i)}(0, t)=v_{0}, \\
& v_{x}^{(i)}(L, t)=\left.A(L) E(L) \frac{\partial v_{x}^{(i)}\left(x^{\prime}, t\right)}{\partial x^{\prime}}\right|_{x^{\prime}=L},
\end{aligned}
$$

which is the strong formulation for axial deformation.

Weak formulation. In the weighted residuals method, the Equation 4.5 is multiplied by an arbitrary function $\omega\left(x^{\prime}\right)$, the so-called weight function, and integrated over the pertinent region:

$$
\int_{0}^{L} \omega(x)\left[\frac{\partial}{\partial x}\left(A E \frac{\partial v_{x}^{(i)}(x, t)}{d x}\right)+b\right] d x=0, \quad 0 \leq x \leq L .
$$

Using integration by parts of the first term to obtain the same derivative of the weight 
function and primary variable $v$ yields:

$$
\int_{0}^{L} \frac{d \omega(x)}{d x}\left(A E \frac{d v_{x}^{(i)}(x, t)}{d x}\right) d x=\underbrace{\left[\omega(x) A E \frac{d v_{x}^{(i)}(x, t)}{d x}\right]_{x=L}^{x=0}}_{\text {boundary conditions }}+\underbrace{\int_{0}^{L} \omega(x) b d x}_{\text {distributed load }} .
$$

Domain discretization. Let's assume nodal values to be known, as shown in Figure 15. Let's also assume that the axial deformation can be approximated by a linear function in the element, which will be function of the nodal deformations.

Figure 15: Interpolation of axial displacement.

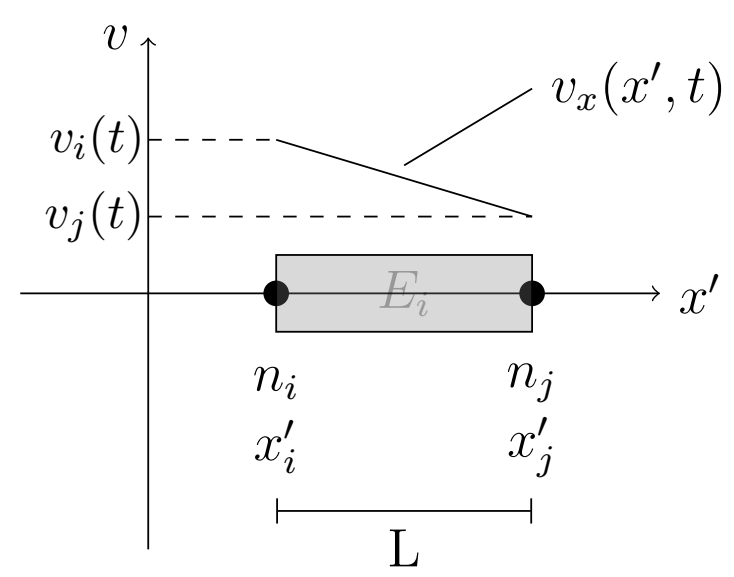

Source: Author.

The axial deformation is then:

$$
v_{x}^{(i)}\left(x^{\prime}, t\right)=-\frac{1}{L}\left(x^{\prime}-x_{j}^{\prime}\right) v_{i}(t)+\frac{1}{L}\left(x^{\prime}-x_{i}^{\prime}\right) v_{j}(t),
$$

and in matrix notation:

$$
v_{x}^{(i)}\left(x^{\prime}, t\right)=\mathbf{N}\left(x^{\prime}\right) \mathbf{d}(t), \quad \mathbf{N}\left(x^{\prime}\right)=\left[\begin{array}{ll}
\underbrace{\frac{1}{L}\left(x^{\prime}-x_{j}^{\prime}\right)}_{N_{1}\left(x^{\prime}\right)} & \underbrace{\frac{1}{L}\left(x^{\prime}-x_{i}^{\prime}\right)}_{N_{2}\left(x^{\prime}\right)}
\end{array}\right], \quad \mathbf{d}(t)=\left[\begin{array}{l}
v_{1}(t) \\
v_{7}(t)
\end{array}\right],
$$

where $\mathbf{N}\left(x^{\prime}\right)$ is the so-called vector of shape functions and $\mathbf{d}(t)$ is the vector of nodal values. 
Writing $v_{x}^{(i)}$ in terms of all the twelve d.o.f.'s (see Figure 12, one has:

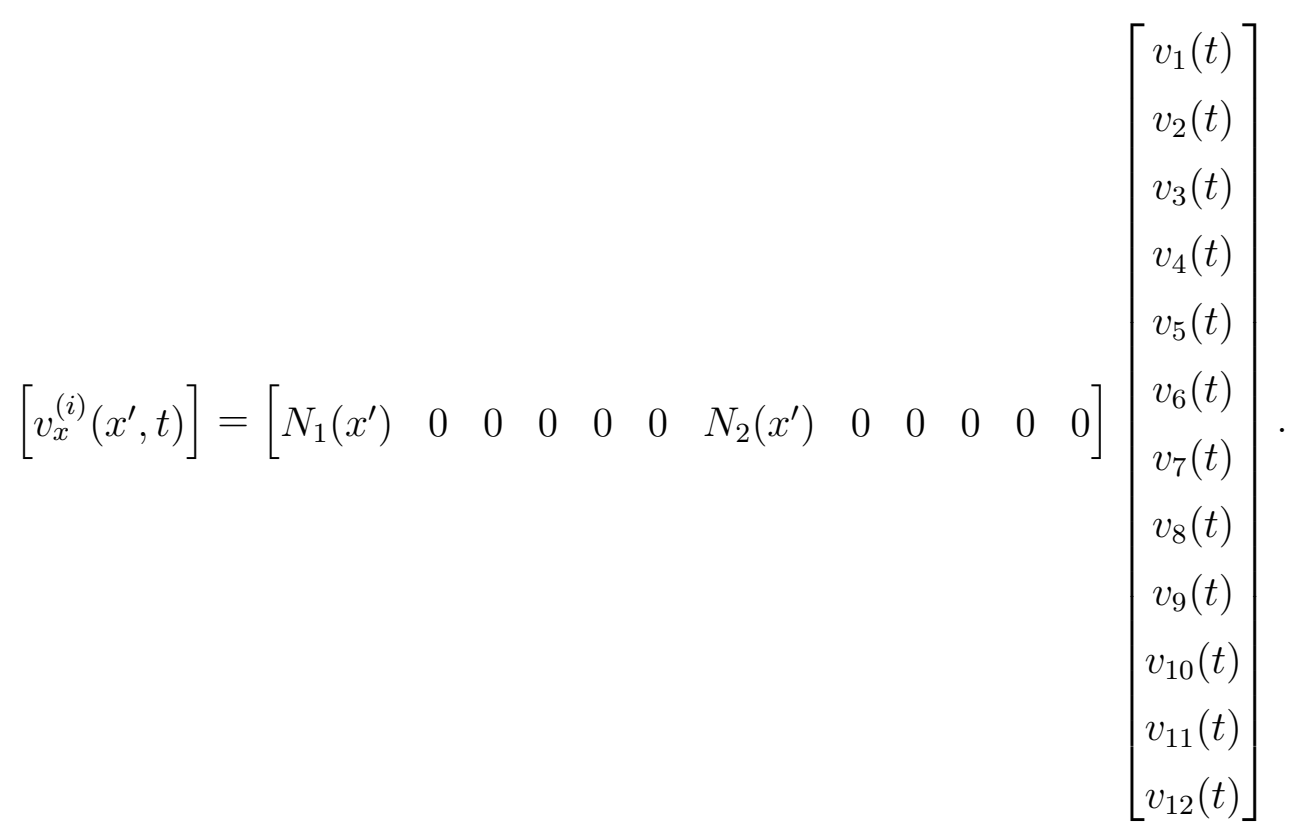

For the next derivations, the arguments $x^{\prime}$ and $t$ are omitted for the sake of readability.

\subsubsection{Bending deformation in the xy-plane, $v_{y}^{(i)}$}

Consider for the bending deformation, $v_{y}^{(i)}$, that depends on the d.o.f.'s $v_{2}, v_{6}, v_{8}$ and $v_{12}$, as shown in Figure 16 .

Figure 16: The d.o.f. for bending displacements in xy-plane.

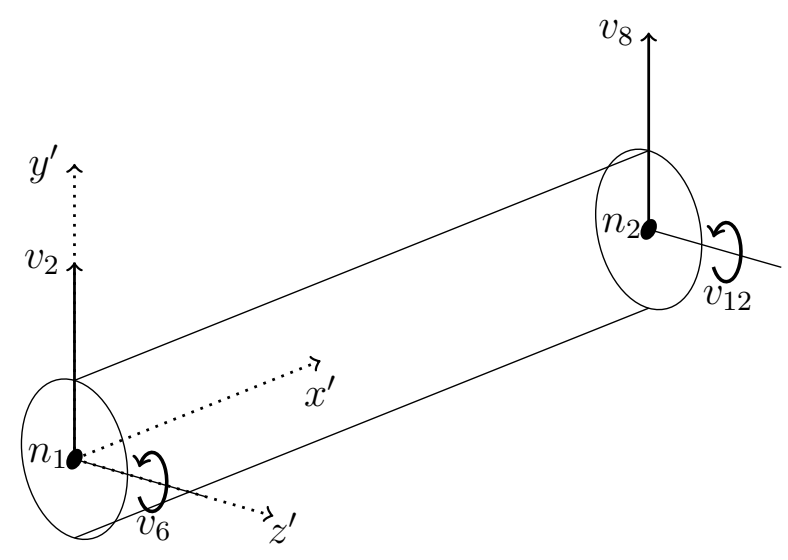

Source: Author.

Strong formulation. Lets define the following variables:

- $M$ : bending moment $[N m]$ 
- $V$ : shear force $[N]$

- $q$ : applied distributed force $[N]$

- E: Young's modulus $\left[N / m^{2}\right]$

- $I_{z}$ : second-moment of inertia $\left[N / m^{2}\right]$

- $\sigma_{x x}$ : uniaxial state of stress $\left[N / m^{2}\right]$

- $\epsilon_{x x}: \operatorname{strain}[-]$

- $\theta_{z}$ : rotation $[\mathrm{rad}]$

- $\kappa$ : curvature $[-]$

According to the Figure 17, the vertical equilibrium equation of an infinitesimal portion is obtained by:

$$
q d x^{\prime}-V+V+d V=0 \Longrightarrow \frac{d V}{d x^{\prime}}=q
$$

Momentum equilibrium around left end, counter clockwise:

$$
M+q d x^{\prime} \frac{d x^{\prime}}{2}+(V+d V) d x^{\prime}-(M+d M)=0
$$

and with the second order terms disregarded, one has:

$$
\frac{d M}{d x^{\prime}}=V
$$

Combining the equilibrium Equation 4.12 and Equation 4.14 .

$$
\frac{d^{2} M}{d x^{\prime 2}}+q=0
$$

Figure 17: Bending load diagram.

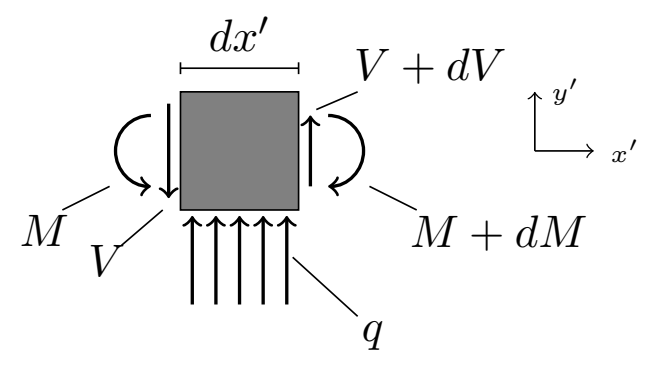

Source: Author. 
The kinematic relations, both rotation and curvature, are:

$$
\theta_{z}^{(i)} \approx \frac{d v_{y}^{(i)}}{d x^{\prime}}, \quad \kappa \approx \frac{d \theta_{z}^{(i)}}{d x^{\prime}}=\frac{d^{2} v_{y}^{(i)}}{d x^{\prime 2}} .
$$

From the constitutive relation for the uniaxial state of stress given by $\sigma_{x x}=E \epsilon_{x x}$, the moment-displacement relation for homogeneous material is given as:

$$
M=-E I_{z} \kappa=-E I \frac{d^{2} v_{y}^{(i)}}{d x^{\prime 2}}
$$

and the strong formulation in terms of displacements is given as:

$$
\frac{d^{2}}{d x^{\prime 2}}\left(E I_{z} \frac{d^{2} v_{y}^{(i)}}{d x^{\prime 2}}\right)-q=0
$$

The assumptions in the formulation are:

- rotation is taken as first derivative of displacement. This is only approximately true if the displacements are small.

- the shear strain is assumed equal to zero which gives zero shear stress. This is not exact but comes out of simplifying a 3D problem to $2 \mathrm{D}$. We will not be concerned about this inconsistency.

- the beam axis is located at the so-called neutral axis where an evenly distribution of normal stresses don't introduce a moment. This results in the axial and bending problem decouples and can be considered separately.

Weak formulation. Multiplying by an arbitrary field (weight function) and integrating over the element, one obtains:

$$
\int_{0}^{L} \omega \frac{d^{2}}{d x^{\prime 2}}\left(E I_{z} \frac{d^{2} v_{y}^{(i)}}{d x^{\prime 2}}\right) d x^{\prime}-\int_{0}^{L} \omega q d x^{\prime}=0
$$

and integrating by parts yields:

$$
-[\omega V]_{0}^{L}-\int_{0}^{L} \frac{d \omega}{d x^{\prime}} \frac{d}{d x^{\prime}}\left(E I_{z} \frac{d^{2} v_{y}^{(i)}}{d x^{\prime 2}}\right) d x^{\prime}-\int_{0}^{L} \omega q d x^{\prime}=0 .
$$


Integrating by parts one more time:

$$
\int_{0}^{L} \frac{d^{2} \omega}{d x^{\prime 2}} E I_{z} \frac{d^{2} v_{y}^{(i)}}{d x^{\prime 2}} d x=\underbrace{[\omega V]_{0}^{L}-\left[\frac{d \omega}{d x^{\prime}} M\right]_{0}^{L}}_{\text {boundary conditions }}+\underbrace{\int_{0}^{L} \omega q d x^{\prime}}_{\text {distributed load }}=0 .
$$

Domain discretization. Let us assume the nodal values to be known. The approximation for the deflection must be able to produce a constant deflection and curvature, i.e. it should at least be twice differentiable. The second derivative of the displacements enters the formulation, hence the first derivative should be continuous over the element boundaries ( $C^{1}$-continuity), or the second derivative will be infinite. It has four node values available, i.e. four shape functions giving the deformation shape. The shape functions are:

$$
v_{y}^{(i)}=\mathbf{N d}, \quad \mathbf{N}=\left[\begin{array}{llll}
N_{3} & N_{4} & N_{5} & N_{6}
\end{array}\right], \quad \mathbf{d}=\left[\begin{array}{c}
v_{2} \\
v_{6} \\
v_{8} \\
v_{12}
\end{array}\right]
$$

and

$$
\theta_{z}=\frac{d v_{y}^{(i)}}{d x^{\prime}}=\frac{d \mathbf{N}}{d x^{\prime}} \mathbf{d}
$$

Considering the boundary conditions at the end of the element, the Hermite Cubic shape equations are:

$$
\begin{gathered}
N_{3}\left(x^{\prime}\right)=1-3 \frac{x^{\prime 2}}{L^{2}}+2 \frac{x^{\prime 3}}{L^{2}}, \\
N_{4}\left(x^{\prime}\right)=-x^{\prime}+2 \frac{x^{\prime 2}}{L}-2 \frac{x^{\prime 3}}{L^{2}}, \\
N_{5}\left(x^{\prime}\right)=3 \frac{x^{\prime 2}}{L^{2}}-2 \frac{x^{\prime 3}}{L^{3}}, \\
N_{6}\left(x^{\prime}\right)=\frac{x^{\prime 2}}{L}-\frac{x^{\prime 3}}{L^{2}} .
\end{gathered}
$$

Writing in the same manner as in Equation 4.11, one has (including $v_{x}$ equation):

$$
\left[\begin{array}{c}
v_{x}^{(i)} \\
v_{y}^{(i)}
\end{array}\right]=\left[\begin{array}{cccccccccccc}
N_{1} & 0 & 0 & 0 & 0 & 0 & N_{2} & 0 & 0 & 0 & 0 & 0 \\
0 & N_{3} & 0 & 0 & 0 & N_{4} & 0 & N_{5} & 0 & 0 & 0 & N_{6}
\end{array}\right]\left[\begin{array}{c}
v_{1} \\
\vdots \\
v_{12}
\end{array}\right]
$$




\subsubsection{Bending deformation in the xz-plane, $v_{z}^{(i)}$}

The case of bending deformation in xz-plane, $v_{z}^{(i)}$, that depends on the d.o.f.'s $v_{3}, v_{5}$, $v_{9}$ and $v_{11}$, is shown in Figure 18 .

Figure 18: The d.o.f. for bending displacements in xz-plane.

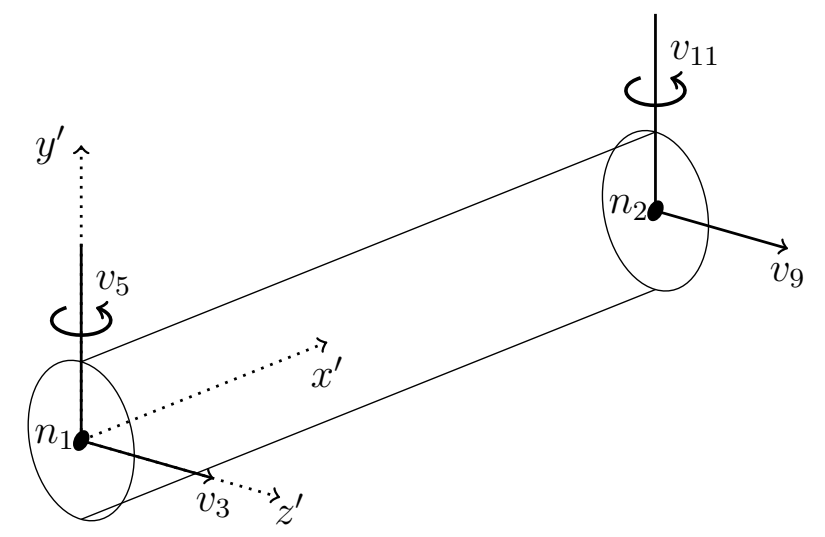

Source: Author.

The derivation of the bending deformation in xz-plane is equal to the xy-plane, only taking care with the direction signal of the forces. Therefore, the procedure is omitted and $v_{z}$ is given by:

$$
v_{z}^{(i)}=\mathbf{N d}, \quad \mathbf{N}=\left[\begin{array}{llll}
N_{3} & -N_{4} & N_{5} & -N_{6}
\end{array}\right], \quad \mathbf{d}=\left[\begin{array}{c}
v_{3} \\
v_{5} \\
v_{9} \\
v_{11}
\end{array}\right],
$$


which will result in:

$$
\left[\begin{array}{l}
v_{x}^{(i)} \\
v_{y}^{(i)} \\
v_{z}^{(i)}
\end{array}\right]=\left[\begin{array}{cccccccccccc}
N_{1} & 0 & 0 & 0 & 0 & 0 & N_{2} & 0 & 0 & 0 & 0 & 0 \\
0 & N_{3} & 0 & 0 & 0 & N_{4} & 0 & N_{5} & 0 & 0 & 0 & N_{6} \\
0 & 0 & N_{3} & 0 & -N_{4} & 0 & 0 & 0 & N_{5} & 0 & -N_{6} & 0
\end{array}\right]\left[\begin{array}{c}
v_{1} \\
v_{2} \\
v_{3} \\
v_{4} \\
v_{5} \\
v_{6} \\
v_{7} \\
v_{8} \\
v_{9} \\
v_{10} \\
v_{11} \\
v_{12}
\end{array}\right] .
$$

\subsubsection{Torsional deformation, $\theta_{x}^{(i)}$}

The torsional deformation, $\theta_{x}^{(i)}$, that depends on the d.o.f.'s $v_{4}$ and $v_{10}$, is shown in Figure 19 .

Figure 19: The d.o.f. for torsion.

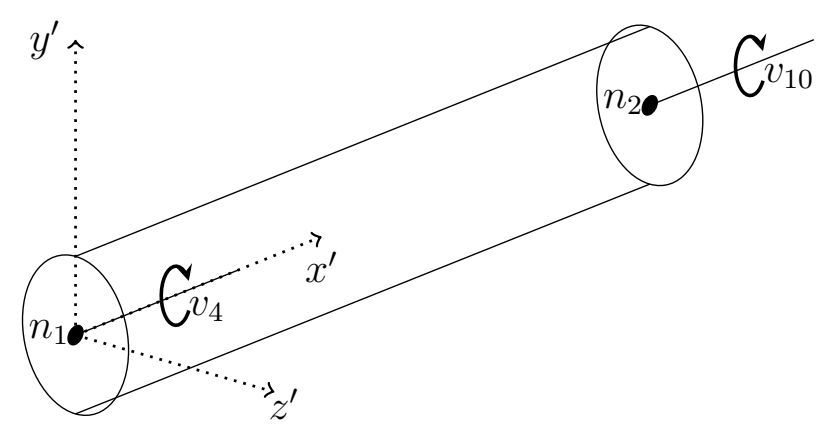

Source: Author.

From the Saint-Venant theory of torsion, the torsional stiffness is given by:

$$
M_{x}=G I_{x} \frac{d \theta_{x}^{(i)}}{d x^{\prime}},
$$

where $M_{x}$ is the twisting moment, $\theta_{x}^{(i)}$ is the torsion angle, $G$ is the shear modulus and $I_{x}$ is torsional moment of inertia. For more information about Saint-Venant theory see chapter seven of (IRGENS, 2008). Considering isotropic materials, the shear modulus is 
given as:

$$
G=\frac{E}{2(1+\nu)}
$$

where $\nu$ is the Poisson's ratio of the material.

The FEM formulation for torsion is identical to the axial formulation. The torsion equation is similar to Equation 4.5 and is given by:

$$
\frac{\partial}{\partial x^{\prime}}\left(G I_{x} \frac{\partial \theta_{x}^{(i)}\left(x^{\prime}, t\right)}{\partial x^{\prime}}\right)+m_{x}=0, \quad 0 \leq x^{\prime} \leq L,
$$

where $m_{x}$ is a distributed twisting load. A linear approximation for the torsion between the nodes are applied using the same shape functions as for axial formulation:

$$
\theta_{x}^{(i)}=\mathbf{N d}, \quad \mathbf{N}=\left[\begin{array}{ll}
\underbrace{-\frac{1}{L}\left(x^{\prime}-x_{j}^{\prime}\right)}_{N_{1}\left(x^{\prime}\right)} & \underbrace{\frac{1}{L}\left(x^{\prime}-x_{i}^{\prime}\right)}_{N_{2}\left(x^{\prime}\right)}
\end{array}\right], \quad \mathbf{d}=\left[\begin{array}{c}
v_{4} \\
v_{10}
\end{array}\right] .
$$

Attaching $\theta$ in terms of all the twelve d.o.f.'s, one has:

$$
\left[\begin{array}{c}
v_{x}^{(i)} \\
v_{y}^{(i)} \\
v_{z}^{(i)} \\
\theta_{x}^{(i)}
\end{array}\right]=\left[\begin{array}{cccccccccccc}
N_{1} & 0 & 0 & 0 & 0 & 0 & N_{2} & 0 & 0 & 0 & 0 & 0 \\
0 & N_{3} & 0 & 0 & 0 & N_{4} & 0 & N_{5} & 0 & 0 & 0 & N_{6} \\
0 & 0 & N_{3} & 0 & -N_{4} & 0 & 0 & 0 & N_{5} & 0 & -N_{6} & 0 \\
0 & 0 & 0 & N_{1} & 0 & 0 & 0 & 0 & 0 & N_{2} & 0 & 0
\end{array}\right]\left[\begin{array}{c}
v_{1} \\
v_{2} \\
v_{3} \\
v_{4} \\
v_{5} \\
v_{6} \\
v_{7} \\
v_{8} \\
v_{9} \\
v_{10} \\
v_{11} \\
v_{12}
\end{array}\right] .
$$

The other rotations, $\theta_{y}^{(i)}$ and $\theta_{z}^{(i)}$, comes from the curvature of the displacements $v_{z}^{(i)}$ and $v_{y}^{(i)}$, respectively:

$$
\left[\begin{array}{c}
\theta_{y}^{(i)} \\
\theta_{z}^{(i)}
\end{array}\right]=\left[\begin{array}{c}
\frac{d v_{z}^{(i)}}{d x} \\
\frac{d v_{y}^{(i)}}{d x}
\end{array}\right]
$$

and the displacements of the $i$ th element, $y^{(i)}\left(x^{\prime}, t\right)$, is discretized and defined by: 
$y^{(i)}\left(x^{\prime}, t\right)=\left[\begin{array}{l}v_{x}^{(i)}\left(x^{\prime}, t\right) \\ v_{y}^{(i)}\left(x^{\prime}, t\right) \\ v_{z}^{(i)}\left(x^{\prime}, t\right) \\ \theta_{x}^{(i)}\left(x^{\prime}, t\right) \\ \theta_{y}^{(i)}\left(x^{\prime}, t\right) \\ \theta_{z}^{(i)}\left(x^{\prime}, t\right)\end{array}\right]=$

$$
\begin{aligned}
& =\left[\begin{array}{cccccccccccc}
N_{1} & 0 & 0 & 0 & 0 & 0 & N_{2} & 0 & 0 & 0 & 0 & 0 \\
0 & N_{3} & 0 & 0 & 0 & N_{4} & 0 & N_{5} & 0 & 0 & 0 & N_{6} \\
0 & 0 & N_{3} & 0 & -N_{4} & 0 & 0 & 0 & N_{5} & 0 & -N_{6} & 0 \\
0 & 0 & 0 & N_{1} & 0 & 0 & 0 & 0 & 0 & N_{2} & 0 & 0 \\
0 & 0 & \frac{d N_{3}}{d x^{\prime}} & 0 & \frac{-d N_{4}}{d x^{\prime}} & 0 & 0 & 0 & \frac{d N_{5}}{d x^{\prime}} & 0 & \frac{-d N_{6}}{d x^{\prime}} & 0 \\
0 & \frac{d N_{3}}{d x^{\prime}} & 0 & 0 & 0 & \frac{d N_{4}}{d x^{\prime}} & 0 & \frac{d N_{5}}{d x^{\prime}} & 0 & 0 & 0 & \frac{d N_{6}}{d x^{\prime}}
\end{array}\right]\left[\begin{array}{c}
v_{1} \\
v_{2} \\
v_{3} \\
v_{4} \\
v_{5} \\
v_{6} \\
v_{7} \\
v_{8} \\
v_{9} \\
v_{10} \\
v_{11} \\
v_{12}
\end{array}\right] \\
& =\mathbf{N}\left(x^{\prime}\right) \mathbf{v}(t) .
\end{aligned}
$$

Therefore, as seen for the global case in the previous chapter, the mass and stiffness matrices for the $i$ th element are given by:

$$
\begin{aligned}
& m_{v}^{(i)}=\int_{0}^{L} \rho \mathbf{N}^{T}\left(x^{\prime}\right) \mathbf{N}\left(x^{\prime}\right) \mathrm{d} x^{\prime}, \\
& k_{v}^{(i)}=\int_{0}^{L} E I \mathbf{N}^{\prime \prime T}\left(x^{\prime}\right) \mathbf{N}^{\prime \prime}\left(x^{\prime}\right) \mathrm{d} x^{\prime} .
\end{aligned}
$$

\subsection{Coordinate transformation}

A major purpose of coordinate transformation is to allow material or element properties, known with reference to one coordinate system or set of nodal d.o.f.'s, to be used in another coordinate system or with reference to another set of nodal d.o.f. (COOK et al. 1974). Let the transformation matrix to be denoted by $R$. 
Let two cartesian reference frames $x y z$ and $x^{\prime} y^{\prime} z^{\prime}$ be arbitrarily oriented with respect to each other, as shown in Figure 20. Also listed in the figure are the cosines of the angles between the various axles (represented by letters $l, m$ and $n$ ). Any variable may be expressed in either coordinate system, as in $x y z$ or $x^{\prime} y^{\prime} z^{\prime}$, both system related by transformation matrix $R$.

Figure 20: Cartesian coordinate systems and table of direction cosines.

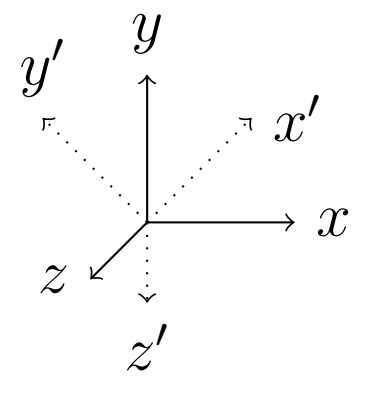

\begin{tabular}{c|ccc} 
& $x$ & $y$ & $z$ \\
\hline$x^{\prime}$ & $l_{1}$ & $m_{1}$ & $n_{1}$ \\
$y^{\prime}$ & $l_{2}$ & $m_{2}$ & $n_{2}$ \\
$z^{\prime}$ & $l_{3}$ & $m_{3}$ & $n_{3}$
\end{tabular}

Source: Author.

\subsubsection{Transformation of element stiffness in two dimensions}

For two-dimensional case, the relation of transformation between the frames $x y$ and $x^{\prime} y^{\prime}$ is shown in the Figure 21 .

Figure 21: Coordinates and direction cosines, two-dimensional case.

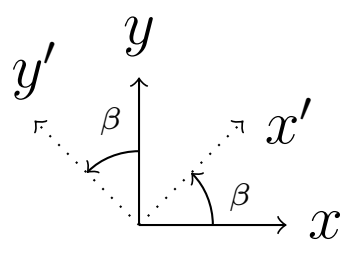

$$
\begin{array}{c|ccc} 
& x & y & z \\
\hline x^{\prime} & l_{1}=\cos \beta & m_{1}=\sin \beta & n_{1}=0 \\
y^{\prime} & l_{2}=-\sin \beta & m_{2}=\cos \beta & n_{2}=0 \\
z^{\prime} & l_{3}=0 & m_{3}=0 & n_{3}=1
\end{array}
$$

Source: Author.

Consider a frame element. The element stiffness matrix $k^{\prime}$ is easily written with reference to a local axis $x^{\prime}$, which coincides with the member axis, as shown in Figure 22 . The problem considered is that of transforming $k^{\prime}$ to $k$ in $x y$ coordinates. In other words, nodal d.o.f. must be changed from $v_{x_{i}}^{\prime}, v_{y_{i}}^{\prime}$ and $\theta_{i}^{\prime}$ to $v_{x_{i}}, v_{y_{i}}$ and $\theta_{i}$, where in this case is $i=1,2$. The $x^{\prime} y^{\prime}$ is called local coordinate system and the $x y$ is called global coordinate system. Therefore, the nodal d.o.f. are related in the two coordinate systems 
by the following equations:

$$
\begin{aligned}
& v_{x_{i}}^{\prime}=v_{x_{i}} \cos \phi+v_{y_{i}} \sin \phi, \\
& v_{y_{i}}^{\prime}=-v_{x_{i}} \sin \phi+v_{y_{i}} \cos \phi, \\
& \theta_{i}^{\prime}=\theta_{i} .
\end{aligned}
$$

Figure 22: 2D Frame element.

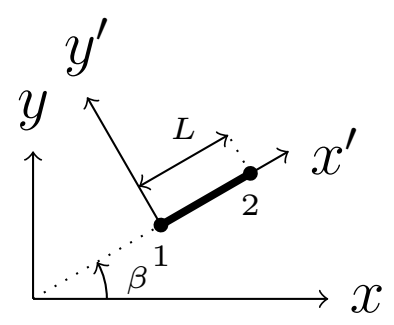

Source: Author.

Let's consider the nodal forces with respect to the two coordinate systems as $f$ and $f^{\prime}$. The relation between them is exactly the same as the two sets of nodal displacements in Equation 4.39, Let's the nodal d.o.f. and forces be arrayed as:

$$
\begin{aligned}
& d=\left[\begin{array}{llllll}
v_{x_{1}} & v_{y_{1}} & \theta_{1} & v_{x_{2}} & v_{y_{2}} & \theta_{2}
\end{array}\right], \\
& f=\left[\begin{array}{llllll}
f_{x_{1}} & f_{y_{1}} & M_{1} & f_{x_{2}} & f_{y_{2}} & M_{2}
\end{array}\right],
\end{aligned}
$$

where $f_{x_{i}}$ is the force in $x$ direction, $f_{y_{i}}$ is the force in $y$ direction, and $M_{i}$ is the moment around the nodes. From the Equations 4.39 , the primed quantities are written as:

$$
d^{\prime}=R d, \quad f^{\prime}=R f
$$

in which:

$$
R=\left[\begin{array}{cc}
\Lambda & 0 \\
0 & \Lambda
\end{array}\right], \quad \Lambda=\left[\begin{array}{ccc}
\cos \phi & \sin \phi & 0 \\
-\sin \phi & \cos \phi & 0 \\
0 & 0 & 1
\end{array}\right]
$$

The transformation matrix $R$ is orthogonal, that is, its inverse is the same as its transpose, $R^{-1}=R^{T}$. This property made easy the inverse calculation, where:

$$
d=R^{T} d^{\prime}, \quad f=R^{T} f^{\prime}
$$

The latter relation is useful in transforming element nodal forces to global directions prior to the assembly of elements. The relation $d^{\prime}=R d$ is useful in extracting local 
displacements from global displacements for the purpose of stress computation COOK et al., 1974).

The transformation of the stiffness matrix follows by substitution of Equations 4.41 in 4.43 and the stiffness relation $k^{\prime} d^{\prime}=f^{\prime}$ into the stiffness relation $k d=f$ :

$$
k d=f=R^{T} f^{\prime}=R^{T} k^{\prime} d^{\prime}=R^{T} k^{\prime} R d .
$$

Then, as this relation is valid for all $d$, the global stiffness matrix is given by:

$$
k=R^{T} k^{\prime} R
$$

\subsubsection{Transformation of element stiffness in three dimensions}

In Figure 23, the beam element 1-2 is presumed to lie in $x^{\prime}$ axle. In order to define the direction of the axles $y^{\prime}$ and $z^{\prime}$, it is used the node 3 as auxiliary node, which is presumed to be in the $x^{\prime} y^{\prime}$ plane.

Figure 23: Arbitrary oriented 3D frame element.

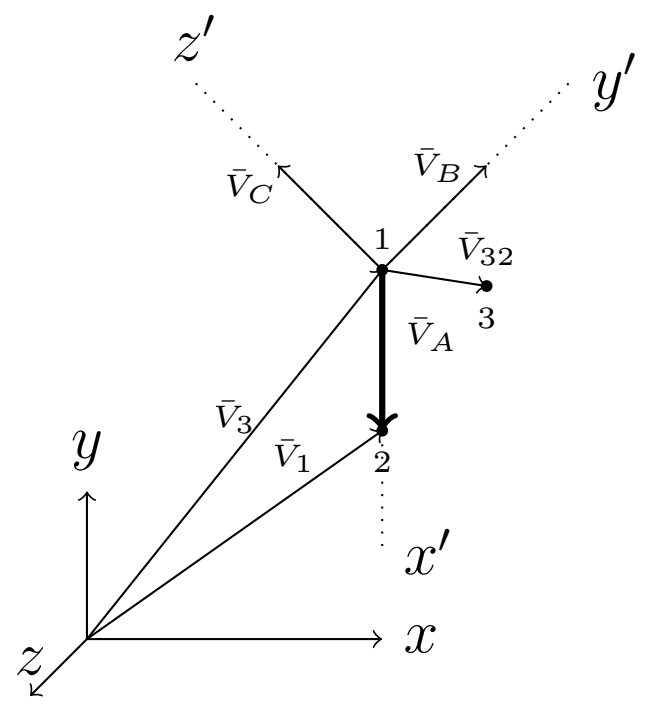

Source: Author.

In order to transform the beam characteristics from the coordinate system $x^{\prime} y^{\prime} z^{\prime}$ to $x y z$, direction cosines must be established. This may be done as follows. Using global coordinates of the nodes 1 and 2 , it is established the vector $\bar{V}_{A}$ :

$$
\bar{V}_{A}=\left[\begin{array}{lll}
\left(x_{1}-x_{2}\right) & \left(y_{1}-y_{2}\right) & \left(z_{1}-z_{2}\right)
\end{array}\right]
$$

Similarly, vector $\bar{V}_{32}$ may be established. Another vector $\bar{V}_{C}$ in direction $z^{\prime}$ is given by 
the cross product:

$$
\bar{V}_{C}=\bar{V}_{A} \times \bar{V}_{32}=\left[\begin{array}{c}
\left(y_{1}-y_{2}\right)\left(z_{2}-z_{3}\right)-\left(y_{2}-y_{3}\right)\left(z_{1}-z_{2}\right) \\
\left(z_{1}-z_{2}\right)\left(x_{2}-z_{3}\right)-\left(z_{2}-z_{3}\right)\left(x_{1}-x_{2}\right) \\
\left(x_{1}-x_{2}\right)\left(y_{2}-y_{3}\right)-\left(x_{2}-x_{3}\right)\left(y_{1}-y_{2}\right)
\end{array}\right] .
$$

Vector $\bar{V}_{B}$ is given by the cross product $\bar{V}_{B}=\bar{V}_{C} \times \bar{V}_{A}$. When vectors $\bar{V}_{A}, \bar{V}_{B}$ and $\bar{V}_{C}$ are normalized by dividing each one by its magnitude, the components of the resulting unit vectors are the direction cosines shown in Figure 20. The transformation is most easily explained if element matrices are first expanded by adding rows and columns of zeros. Let the local and global displacements vectors be, respectively:

$$
\begin{aligned}
d^{\prime} & =\left[\begin{array}{llllllllllll}
v_{x_{1}}^{\prime} & v_{y_{1}}^{\prime} & v_{z_{1}}^{\prime} & \theta_{x_{1}}^{\prime} & \theta_{y_{1}}^{\prime} & \theta_{z_{1}}^{\prime} & v_{x_{2}}^{\prime} & v_{y_{2}}^{\prime} & v_{z_{2}}^{\prime} & \theta_{x_{2}}^{\prime} & \theta_{y_{2}}^{\prime} & \theta_{z_{2}}^{\prime}
\end{array}\right] \\
d & =\left[\begin{array}{llllllllllll}
v_{x_{1}} & v_{y_{1}} & v_{z_{1}} & \theta_{x_{1}} & \theta_{y_{1}} & \theta_{z_{1}} & v_{x_{2}} & v_{y_{2}} & v_{z_{2}} & \theta_{x_{2}} & \theta_{y_{2}} & \theta_{z_{2}}
\end{array}\right] .
\end{aligned}
$$

The transformation of the displacements $v$ and rotations $\theta$ are decoupled. Pursuing these arguments, it is obtained the transformations given by Equations 4.41, 4.43, and 4.45, in which:

$$
R=\left[\begin{array}{llll}
\Lambda & 0 & 0 & 0 \\
0 & \Lambda & 0 & 0 \\
0 & 0 & \Lambda & 0 \\
0 & 0 & 0 & \Lambda
\end{array}\right], \quad \Lambda=\left[\begin{array}{lll}
l_{1} & m_{1} & n_{1} \\
l_{2} & m_{2} & n_{2} \\
l_{3} & m_{3} & n_{3}
\end{array}\right]
$$

Again, $R$ is orthogonal, that is, $R^{T} R=I$. This may be seen by noting that the columns of $\Lambda$ form an orthogonal triple of unit vectors.

\subsection{Assembly of elements}

The Equation 4.37 corresponds to the $i$ th element, which has two nodes with six d.o.f. per node, as shown in Figure 12. However, the system is composed by several elements that defines the geometric domain of the model. It is then necessary to assembly all the elements in order to form the complete bar. The assemblage process for obtaining the structure stiffness matrix $K_{v}$ is symbolically written:

$$
K_{v}=\sum_{i} k_{v}^{(i)}
$$


where the matrix $k_{v}^{(i)}$ is the stiffness matrix of the $i$ th element and the summation goes over all elements in the assemblage. In an analogous manner the structure mass matrix $M_{v}$ and load vectors are assembled from the element mass matrices $m_{v}^{(i)}$ and element load vectors, respectively. In addition to the element stiffness, mass and load matrices, concentrated stiffness, masses and loads corresponding to specific d.o.f. can also be added (BATHE, 2006).

Consider a beam element with two nodes, which the element stiffness can be written generically as:

$$
k_{v}^{(i)}=\left[\begin{array}{cc}
K_{11}^{(i)} & K_{12}^{(i)} \\
K_{21}^{(i)} & K_{22}^{(i)}
\end{array}\right],
$$

then, the global stiffness matrix is given by:

$$
K_{v}=\left[\begin{array}{ccccccc}
K_{11}^{(1)} & K_{12}^{(1)} & 0 & 0 & \cdots & 0 & 0 \\
& K_{22}^{(1)}+K_{11}^{(2)} & K_{12}^{(2)} & 0 & \cdots & 0 & 0 \\
& & K_{22}^{(2)}+K_{11}^{(3)} & K_{12}^{(3)} & \cdots & 0 & 0 \\
\text { symmetric } & & K_{22}^{(3)}+K_{11}^{(4)} & \cdots & 0 & 0 \\
& & & \ddots & 0 & 0 \\
& & & & & K_{22}^{(n-1)}+K_{11}^{(n)} & K_{12}^{(n)} \\
& & & & & & K_{22}^{(n)}
\end{array}\right]
$$

where $n$ is the total number of elements of the model and subscripts 1 and 2 is referring the nodes 1 and 2 of each element. In an analogous manner, the global mass matrix $M_{v}$, the global damping matrix $D_{v}$ and load vectors are assembled for the complete model.

\subsection{Bar geometry for the FEM}

Now, all information needed to solve the Equations 3.63, 3.64 and 3.74 are known and can be implemented using the state-space approach. The Figure 24 shows the spatial discretization of the passive anti-roll bar used in the FEM. It consists of 16 elements, formed by 17 nodes. The Table 2 presents the $x-y-z$ coordinates of the nodes. 
Figure 24: Geometry of the passive anti-roll bar.

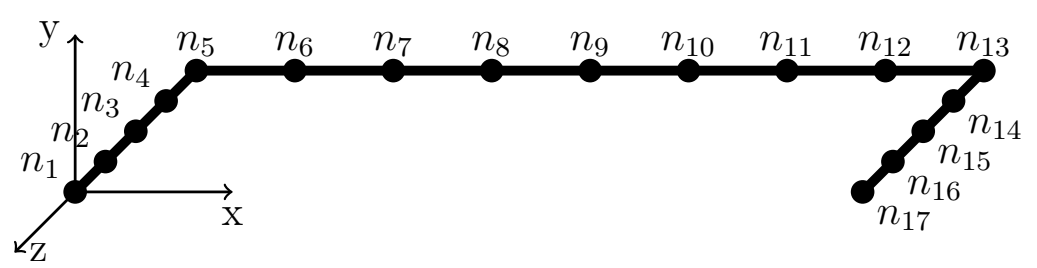

Source: Author.

Table 2: Node's spatial coordinates, $\mathrm{x}-\mathrm{y}-\mathrm{z}$.

\begin{tabular}{|c|c|c|c|}
\hline \multicolumn{4}{|c|}{ Spatial distribution of the nodes } \\
\hline Description & $\mathrm{x}[\mathrm{m}]$ & $\mathrm{y}[\mathrm{m}]$ & $\mathrm{z}[\mathrm{m}]$ \\
\hline$n_{1}$ & 0 & 0 & 0 \\
$n_{2}$ & 0 & 0 & -0.025 \\
$n_{3}$ & 0 & 0 & -0.050 \\
$n_{4}$ & 0 & 0 & -0.075 \\
$n_{5}$ & 0 & 0 & -0.1 \\
$n_{6}$ & 0.15 & 0 & -0.1 \\
$n_{7}$ & 0.30 & 0 & -0.1 \\
$n_{8}$ & 0.45 & 0 & -0.1 \\
$n_{9}$ & 0.60 & 0 & -0.1 \\
$n_{10}$ & 0.75 & 0 & -0.1 \\
$n_{11}$ & 0.90 & 0 & -0.1 \\
$n_{12}$ & 1.05 & 0 & -0.1 \\
$n_{13}$ & 1.20 & 0 & -0.1 \\
$n_{14}$ & 1.20 & 0 & -0.075 \\
$n_{15}$ & 1.20 & 0 & -0.050 \\
$n_{16}$ & 1.20 & 0 & -0.025 \\
$n_{17}$ & 1.20 & 0 & 0 \\
\hline
\end{tabular}

Source: Author.

So far, a methodology for modeling hybrid systems has been presented and applied in the passive anti-roll system, which contains both discrete and distributed parameters. In the next chapter, the same methodology will be used for deriving the active model of the anti-roll bar, in which the passive bar will be divided into two bars connected now by a torque generator, which represents an electric motor that will provide the torque for control of the angle of roll. 


\section{ACTIVE ANTI-ROLL COMPLETE MODEL}

This chapter deals with the active system. Then, the model of the active anti-roll bar will be deduced and added to the roll dynamics model, in the same way as presented in the Chapter 4 for the passive case.

\subsection{Active anti-roll bar}

Consider the system, as shown in Figure 25, which consists of two flexible bars, each one connected to an unsprung mass and an electric motor, which provides the control torque for the new system. As in the previous section, it is desired to seek a set of equations that represents the system, so that the influence of the control torque shows that the roll angle will be minimized by the active system when compared to the passive system. Denote by $y_{A}(x, t)$ the vector of deflections of the bar $A$ and $y_{B}(x, t)$ the vector of deflections of the bar $B$. The kinetic energy $T$ and potential energy $V$ expressions are:

$$
\begin{gathered}
T=\frac{m_{s} \dot{z}_{s}^{2}}{2}+\frac{m_{u r} \dot{y}_{A}^{2}(0, t)}{2}+\frac{m_{u l} \dot{y}_{B}^{2}(L, t)}{2}+\frac{J_{s} \dot{\phi}^{2}}{2} \\
+\frac{1}{2} \int_{0}^{L} \rho \dot{y}_{A}^{2}(x, t) \mathrm{d} x+\frac{1}{2} \int_{0}^{L} \rho \dot{y}_{B}^{2}(x, t) \mathrm{d} x \\
V=m_{s} g h \cos \phi+\frac{k_{t}}{2}\left(y_{A}(0, t)-z_{r r}\right)^{2}+\frac{k_{t}}{2}\left(y_{B}(L, t)-z_{r l}\right)^{2} \\
+\frac{k_{s}}{2}\left(z_{s}-\frac{l}{2} \sin \phi-y_{A}(0, t)\right)^{2}+\frac{k_{s}}{2}\left(z_{s}+\frac{l}{2} \sin \phi-y_{B}(L, t)\right)^{2} \\
+\frac{1}{2} \int_{0}^{L} E I\left(y_{A}^{\prime \prime}(x, t)\right)^{2} \mathrm{~d} x+\frac{1}{2} \int_{0}^{L} E I\left(y_{B}^{\prime \prime}(x, t)\right)^{2} \mathrm{~d} x .
\end{gathered}
$$


Figure 25: Roll dynamics model with active anti-roll bar.

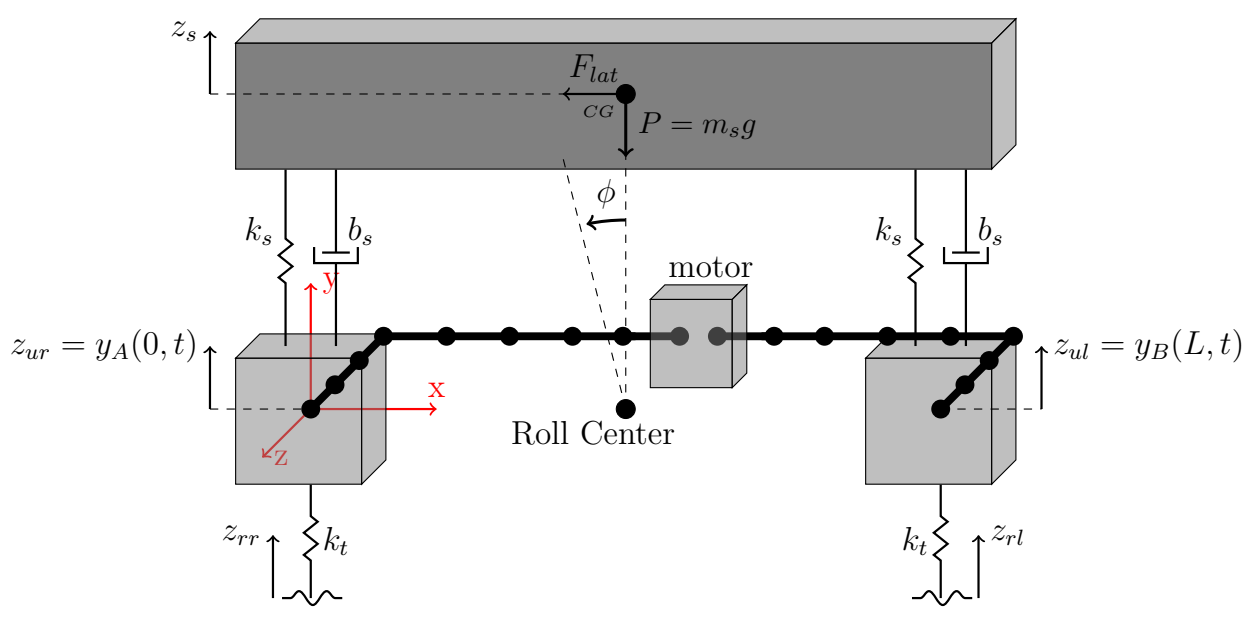

Source: Author.

The Lagrangian expression terms, as shown in Equation 3.30, are:

$$
\begin{gathered}
L_{D}=\frac{J_{s} \dot{\phi}^{2}}{2}+\frac{m_{s} \dot{z}_{s}^{2}}{2}-m_{s} g h \cos \phi-k_{s} z_{s}^{2}-k_{s} \frac{l^{2}}{4} \sin ^{2} \phi \\
\hat{L}=\frac{1}{2} \rho \dot{y}_{A}^{2}(x, t)+\frac{1}{2} \rho \dot{y}_{B}^{2}(x, t)-\frac{1}{2} E I\left(y_{A}^{\prime \prime}(x, t)\right)^{2}-\frac{1}{2} E I\left(y_{B}^{\prime \prime}(x, t)\right)^{2}, \\
L_{B}=\frac{m_{u r} \dot{y}_{A}^{2}(0, t)}{2}+\frac{m_{u l} \dot{y}_{B}^{2}(L, t)}{2}-\frac{k_{t}}{2}\left(y_{A}(0, t)-z_{r r}\right)^{2}-\frac{k_{t}}{2}\left(y_{B}(L, t)-z_{r l}\right)^{2} \\
-\frac{k_{s} y_{A}^{2}(0, t)}{2}-\frac{k_{s} y_{B}^{2}(L, t)}{2}-k_{s} \frac{l}{2} \sin \phi\left(y_{A}(0, t)-y_{B}(L, t)\right) \\
+k_{s} z_{s}\left(y_{A}(0, t)+y_{B}(L, t)\right) .
\end{gathered}
$$

\subsubsection{Non-conservative work $W_{n c}$}

For the active anti-roll system, as it was said, it is added an electric motor that connect both bars $\mathrm{A}$ and $\mathrm{B}$ and it is accountable to generate the control input of the system, i.e., the torsional stiffness control of the set of bars. At first, it is not considered the dynamic model of the motor, being treated as providing a direct control torque. Hence, in addition to the non-conservative work derived for the passive roll bar, it is added the work provided by the motor inserted into the model. The work performed by the external toque is given as:

$$
W_{\text {torque }}=-u \theta_{y_{A}}(L, t)+u \theta_{y_{B}}(0, t)=W_{\text {torque }}\left(\theta_{y_{A}}(L, t), \theta_{y_{B}}(0, t)\right)
$$

where $u$ is the torque applied to the bars $\mathrm{A}$ and $\mathrm{B}$ with opposite directions, as seen in Figure 26. 
Figure 26: Torque direction adopted for the electric motor.

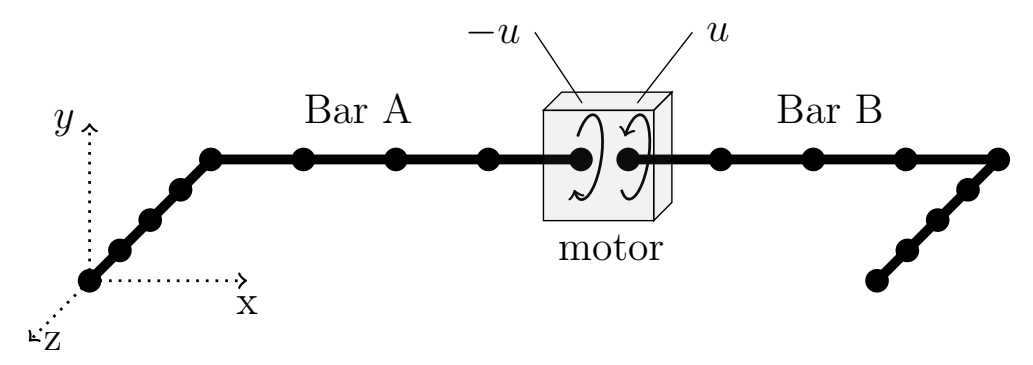

Source: Author.

The conservative energy provided by the dampers are split, each one for each bar. Then, the $W_{\text {fric }}$ is expressed as:

$$
\begin{aligned}
W_{\text {fric }} & =\frac{1}{2} b_{s}\left(\dot{z}_{s}-\frac{l}{2} \dot{\phi} \cos \phi-\dot{y}_{A}(0, t)\right)^{2}+\frac{1}{2} b_{s}\left(\dot{z}_{s}+\frac{l}{2} \dot{\phi} \cos \phi-\dot{y}_{B}(L, t)\right)^{2} \\
& =W_{\text {fric }}\left(\phi, \dot{\phi}, \dot{z}_{s}, \dot{y}_{A}(0, t), \dot{y}_{B}(L, t)\right) .
\end{aligned}
$$

The lateral force work is not changed and, then, the total work of the dissipative energy and external forces, considering the control torque provided by the motor, is given by:

$$
W_{n c}=W_{F_{l a t}}+W_{\text {fric }}+W_{\text {torque }} .
$$

\subsubsection{Equations of motion}

In order to apply the generalized Hamilton's principle, as in Equation 3.29, the variations are taken from the work expressions. In the following, these variations are described.

$$
\begin{gathered}
\delta W_{F_{\text {lat }}}=\frac{\partial W_{F_{\text {lat }}}}{\partial \phi} \delta \phi, \\
\delta W_{\text {fric }}=\frac{\partial W_{\text {fric }}}{\partial \phi} \delta \phi+\frac{\partial W_{\text {fric }}}{\partial \dot{\phi}} \delta \dot{\phi}+\frac{\partial W_{\text {fric }}}{\partial \dot{z}_{s}} \delta \dot{z}_{s}+ \\
+\frac{\partial W_{\text {fric }}}{\partial \dot{y}_{A}(0, t)} \delta \dot{y}_{A}(0, t)+\frac{\partial W_{\text {fric }}}{\partial \dot{y}_{B}(L, t)} \delta \dot{y}_{B}(L, t), \\
\delta W_{\text {torque }}=\frac{\partial W_{\text {torque }}}{\partial \theta_{y_{A}(L, t)}} \delta \theta_{y_{A}(L, t)}+\frac{\partial W_{\text {torque }}}{\partial \theta_{y_{B}(0, t)}} \delta \theta_{y_{B}(0, t)} .
\end{gathered}
$$


Hence, the total non-conservative virtual work is given as:

$$
\begin{aligned}
& \left.\delta W_{n c}=\left[F_{l a t} h \cos \phi+b_{s} \frac{l}{2} \cos \phi\left(\dot{y}_{B}(L, t)-\dot{y}_{A}(0, t)\right)-b_{s} \frac{l^{2}}{2} \dot{\phi} \cos ^{2} \phi\right)\right] \delta \phi \\
& +\left[b_{s}\left(\dot{y}_{A}(0, t)+\dot{y}_{B}(L, t)\right)-2 b_{s} \dot{z}_{s}\right] \delta z_{s}-u \delta \theta_{y_{A}(L, t)}+u \delta \theta_{y_{B}(0, t)} \\
& +\left[b_{s}\left(\dot{z}_{s}-\frac{l}{2} \dot{\phi} \cos \phi-\dot{y}_{A}(0, t)\right)\right] \delta y_{A}(0, t)+\left[b_{s}\left(\dot{z}_{s}+\frac{l}{2} \dot{\phi} \cos \phi-\dot{y}_{B}(L, t)\right)\right] \delta y_{B}(L, t),
\end{aligned}
$$

where $u$ is the torque input applied by the motor.

- Discrete term variation:

$$
\begin{aligned}
\delta L_{D} & =\frac{\partial L_{D}}{\partial \phi} \delta \phi+\frac{\partial L_{D}}{\partial \dot{\phi}} \delta \dot{\phi}+\frac{\partial L_{D}}{\partial z_{s}} \delta z_{s}+\frac{\partial L_{D}}{\partial \dot{z}_{s}} \delta \dot{z}_{s} \\
& =\left[m_{s} g h \sin \phi-k_{s} \frac{l^{2}}{2} \sin \phi \cos \phi\right] \delta \phi+J_{s} \dot{\phi} \delta \dot{\phi}-2 k_{s} z_{s} \delta z_{s}+m_{s} \dot{z}_{s} \delta \dot{z}_{s}
\end{aligned}
$$

- Distributed term variation:

$$
\begin{aligned}
\delta \hat{L}= & \frac{\partial \hat{L}}{\partial y_{A}^{\prime \prime}(x, t)} \delta y_{A}^{\prime \prime}(x, t)+\frac{\partial \hat{L}}{\partial \dot{y}_{A}(x, t)} \delta \dot{y}_{A}(x, t)+\frac{\partial \hat{L}}{\partial y_{B}^{\prime \prime}(x, t)} \delta y_{B}^{\prime \prime}(x, t)+\frac{\partial \hat{L}}{\partial \dot{y}_{B}(x, t)} \delta \dot{y}_{B}(x, t) \\
= & -E I\left[y_{A}^{\prime \prime}(x, t) \delta y_{A}^{\prime \prime}(x, t)+y_{B}^{\prime \prime}(x, t) \delta y_{B}^{\prime \prime}(x, t)\right] \\
& +\rho\left[\dot{y}_{A}(x, t) \delta \dot{y}_{A}(x, t)+\dot{y}_{B}(x, t) \delta \dot{y}_{B}(x, t)\right] .
\end{aligned}
$$

- Boundary term variation:

$$
\begin{aligned}
\delta L_{B}= & \frac{\partial L_{B}}{\partial y_{A}(0, t)} \delta y_{A}(0, t)+\frac{\partial L_{B}}{\partial y_{B}(L, t)} \delta y_{B}(L, t)+\frac{\partial L_{B}}{\partial \dot{y}_{A}(0, t)} \delta \dot{y}_{A}(0, t) \\
& +\frac{\partial L_{B}}{\partial \dot{y}_{B}(L, t)} \delta \dot{y}_{B}(L, t)+\frac{\partial L_{B}}{\partial \phi} \delta \phi+\frac{\partial L_{B}}{\partial z_{s}} \delta z_{s} \\
= & {\left[k_{s} z_{s}-k_{s} \frac{l}{2} \sin \phi-k_{s} y_{A}(0, t)-k_{t}\left(y_{A}(0, t)-z_{r r}\right)\right] \delta y_{A}(0, t) } \\
& +\left[k_{s} z_{s}+k_{s} \frac{l}{2} \sin \phi-k_{s} y_{B}(L, t)-k_{t}\left(y_{B}(L, t)-z_{r l}\right)\right] \delta y_{B}(L, t) \\
& +m_{u r} \dot{y}_{A}(0, t) \delta \dot{y}_{A}(0, t)+m_{u l} \dot{y}_{B}(L, t) \delta \dot{y}_{B}(L, t) \\
& -k_{s} \frac{l}{2} \cos \phi\left(y_{A}(0, t)-y_{B}(L, t)\right) \delta \phi+k_{s}\left[y_{A}(0, t)+y_{B}(L, t)\right] \delta z_{s} .
\end{aligned}
$$

Each term of the generalized Hamilton's principle is obtained as follows:

$$
\int_{t_{1}}^{t_{2}}\left(\delta L_{D}+\int_{\Omega} \delta \hat{L} \mathrm{~d} \Omega+\delta L_{B}+\delta W_{n c}\right) \mathrm{d} t=0 .
$$


- First term: $\int_{t_{1}}^{t_{2}} \delta L_{D} \mathrm{~d} t$

$$
\int_{t_{1}}^{t_{2}}\left\{\left[m_{s} g h \sin \phi-k_{s} \frac{l^{2}}{2} \sin \phi \cos \phi\right] \delta \phi+J_{s} \dot{\phi} \delta \dot{\phi}-2 k_{s} z_{s} \delta z_{s}+m_{s} \dot{z}_{s} \delta \dot{z}_{s}\right\} \mathrm{d} t
$$

and using integration by parts in Equation 5.17 leads to:

$$
\int_{t_{1}}^{t_{2}}\left\{\left[m_{s} g h \sin \phi-k_{s} \frac{l^{2}}{2} \sin \phi \cos \phi-J_{s} \ddot{\phi}\right] \delta \phi-\left[2 k_{s} z_{s}+m_{s} \ddot{z}_{s}\right] \delta z_{s}\right\} \mathrm{d} t .
$$

- Second term: $\int_{t_{1}}^{t_{2}} \int_{\Omega} \delta \hat{L} \mathrm{~d} \Omega \mathrm{d} t$

$$
\begin{aligned}
& \int_{t_{1}}^{t_{2}}\left\{\int_{0}^{L}\left[\rho \dot{y}_{A}(x, t) \delta \dot{y}_{A}(x, t)-E I y_{A}^{\prime \prime}(x, t) \delta y_{A}^{\prime \prime}(x, t)\right] \mathrm{d} x\right. \\
& \left.+\int_{0}^{L}\left[\rho \dot{y}_{B}(x, t) \delta \dot{y}_{B}(x, t)-E I y_{B}^{\prime \prime}(x, t) \delta y_{B}^{\prime \prime}(x, t)\right] \mathrm{d} x\right\} \mathrm{d} t
\end{aligned}
$$

and integrating Equation 5.19 by parts leads to:

$$
\begin{aligned}
& \int_{t_{1}}^{t_{2}}\left\{\int_{0}^{L}\left[-\rho \ddot{y}_{A}(x, t)-E I y_{A}^{\prime \prime \prime \prime}(x, t)\right] \delta y_{A}(x, t) \mathrm{d} x\right. \\
& -\left.E I y_{A}^{\prime \prime}(x, t) \delta y_{A}^{\prime}(x, t)\right|_{x=L} ^{x=0}+\left.E I y_{A}^{\prime \prime \prime}(x, t) \delta y_{A}(x, t)\right|_{x=L} ^{x=0} \\
& +\int_{0}^{L}\left[-\rho \ddot{y}_{B}(x, t)-E I y_{B}^{\prime \prime \prime \prime}(x, t)\right] \delta y_{B}(x, t) \mathrm{d} x \\
& \left.-\left.E I y_{B}^{\prime \prime}(x, t) \delta y_{B}^{\prime}(x, t)\right|_{x=L} ^{x=0}+\left.E I y_{B}^{\prime \prime \prime}(x, t) \delta y_{B}(x, t)\right|_{x=L} ^{x=0}\right\} \mathrm{d} t .
\end{aligned}
$$

- Third term: $\int_{t_{1}}^{t_{2}} \delta L_{B} \mathrm{~d} t$

$$
\begin{aligned}
& \int_{t_{1}}^{t_{2}}\left\{\left[k_{s} z_{s}-k_{s} \frac{l}{2} \sin \phi-k_{s} y_{A}(0, t)-k_{t}\left(y_{A}(0, t)-z_{r r}\right)\right] \delta y_{A}(0, t)\right. \\
& +\left[k_{s} z_{s}+k_{s} \frac{l}{2} \sin \phi-k_{s} y_{B}(L, t)-k_{t}\left(y_{B}(L, t)-z_{r l}\right)\right] \delta y_{B}(L, t) \\
& +m_{u r} \dot{y}_{A}(0, t) \delta \dot{y}_{A}(0, t)+m_{u l} \dot{y}_{B}(L, t) \delta \dot{y}_{B}(L, t) \\
& \left.-k_{s} \frac{l}{2} \cos \phi\left[y_{A}(0, t)-y_{B}(L, t)\right] \delta \phi+k_{s}\left[y_{A}(0, t)+y_{B}(L, t)\right] \delta z_{s}\right\} \mathrm{d} t
\end{aligned}
$$

and integrating Equation 5.21 by parts under the time derivative term variations 
leads to:

$$
\begin{aligned}
& \int_{t_{1}}^{t_{2}}\left\{\left[k_{s} z_{s}-k_{s} \frac{l}{2} \sin \phi-k_{s} y_{A}(0, t)-k_{t}\left(y_{A}(0, t)-z_{r r}\right)\right] \delta y_{A}(0, y)\right. \\
& +\left[k_{s} z_{s}+k_{s} \frac{l}{2} \sin \phi-k_{s} y_{B}(L, t)-k_{t}\left(y_{B}(L, t)-z_{r l}\right)\right] \delta y_{B}(L, y) \\
& \left.-\left[k_{s} \frac{l}{2} \cos \phi\left(y_{A}(0, t)-y_{B}(L, t)\right)\right] \delta \phi+\left[k_{s}\left(y_{A}(0, t)+y_{B}(L, t)\right)\right] \delta z_{s}\right\} \mathrm{d} t .
\end{aligned}
$$

- Fourth term: $\int_{t_{1}}^{t_{2}} \delta W_{n c} \mathrm{~d} t$

$$
\begin{aligned}
& \int_{t_{1}}^{t_{2}}\left\{\left[F_{l a t} h \cos \phi-b_{s} \frac{l}{2} \cos \phi\left(\dot{y}_{B}(L, t)-\dot{y}_{A}(0, t)-l \dot{\phi} \cos \phi\right)\right] \delta \phi\right. \\
& +\left[b_{s}\left(\dot{y}_{A}(0, t)+\dot{y}_{B}(L, t)\right)-2 b_{s} \dot{z}_{s}\right] \delta z_{s}-u \delta \theta_{y_{A}(L, t)}+u \delta \theta_{y_{B}(0, t)} \\
& +\left[b_{s}\left(\dot{z}_{s}-\frac{l}{2} \dot{\phi} \cos \phi-\dot{y}_{A}(0, t)\right)\right] \delta y_{A}(0, t) \\
& +\left[b_{s}\left(\dot{z}_{s}+\frac{l}{2} \dot{\phi} \cos \phi-\dot{y}_{B}(L, t)\right)\right] \delta y_{B}(L, t) .
\end{aligned}
$$

Writing all terms with its respective variations in the extended Hamilton's equation:

$$
\begin{aligned}
& \int_{t_{1}}^{t_{2}}\left\{\left[m_{s} g h \sin \phi-k_{s} \frac{l^{2}}{2} \sin \phi \cos \phi-J_{s} \ddot{\phi}-k_{s} \frac{l}{2} \cos \phi\left(y_{A}(0, t)-y_{B}(L, t)\right)\right.\right. \\
& \left.-b_{s} \frac{l}{2} \cos \phi\left(\dot{y}_{B}(L, t)-\dot{y}_{A}(0, t)-l \dot{\phi} \cos \phi\right)+F_{l a t} h \cos \phi\right] \delta \phi \\
& +\left[b_{s}\left(\dot{y}_{A}(0, t)+\dot{y}_{B}(L, t)\right)-2 b_{s} \dot{z}_{s}-m_{s} \ddot{z}_{s}-2 k_{s} z_{s}+k_{s}\left(y_{A}(0, t)+y_{B}(L, t)\right)\right] \delta z_{s} \\
& -\int_{0}^{L}\left[\rho \ddot{y}_{A}(x, t)+E I y_{A}^{\prime \prime \prime \prime}(x, t)\right] \delta y_{A}(x, t) \mathrm{d} x-\int_{0}^{L}\left[\rho \ddot{y}_{B}(x, t)+E I y_{B}^{\prime \prime \prime \prime}(x, t)\right] \delta y_{B}(x, t) \mathrm{d} x \\
& -\left.E I y_{A}^{\prime \prime}(x, t) \delta y_{A}^{\prime}(x, t)\right|_{x=0} ^{x=L}+\left.E I y_{A}^{\prime \prime \prime}(x, t) \delta y_{A}(x, t)\right|_{x=L} ^{x=0}-u \delta \theta_{y_{A}(L, t)} \\
& +\left[k_{s}\left(z_{s}-\frac{l}{2} \sin \phi-y_{A}(0, t)\right)-k_{t}\left(y_{A}(0, t)-z_{r r}\right)\right. \\
& \left.-m_{u r} \ddot{y}_{A}(L, t)+b_{s}\left(\dot{z}_{s}-\frac{l}{2} \dot{\phi} \cos \phi-\dot{y}_{A}(0, t)\right)\right] \delta y_{A}(0, t) \\
& -\left.E I y_{B}^{\prime \prime}(x, t) \delta y_{B}^{\prime}(x, t)\right|_{x=0} ^{x=L}+\left.E I y_{B}^{\prime \prime \prime}(x, t) \delta y_{B}(x, t)\right|_{x=L} ^{x=0}+u \delta \theta_{y_{B}(0, t)} \\
& +\left[k_{s}\left(z_{s}+\frac{l}{2} \sin \phi-y_{B}(L, t)\right)-k_{t}\left(y_{B}(L, t)-z_{r l}\right)\right. \\
& \left.\left.-m_{u l} \ddot{y}_{B}(L, t)+b_{s}\left(\dot{z}_{s}+\frac{l}{2} \dot{\phi} \cos \phi-\dot{y}_{B}(L, t)\right)\right] \delta y_{B}(L, t)\right\} \mathrm{d} t .
\end{aligned}
$$

Therefore, the equations taken from the generalized Hamilton's principle for the active 
anti-roll bar are:

1. Equation for roll dynamics:

$$
\begin{aligned}
& J_{s} \ddot{\phi}-m_{s} g h \sin \phi+k_{s} \frac{l^{2}}{2} \sin \phi \cos \phi+k_{s} \frac{l}{2} \cos \phi\left(y_{A}(0, t)-y_{B}(L, t)\right) \\
& +b_{s} \frac{l}{2} \cos \phi\left(\dot{y}_{B}(L, t)-\dot{y}_{A}(0, t)-l \dot{\phi} \cos \phi\right)-F_{l a t} h \cos \phi=0 .
\end{aligned}
$$

2. Equation for vertical translation dynamics of the sprung mass:

$$
m_{s} \ddot{z}_{s}+2 k_{s} z_{s}-k_{s}\left(y_{A}(0, t)+y_{B}(L, t)\right)-b_{s}\left(\dot{y}_{A}(0, t)+\dot{y}_{B}(L, t)-2 \dot{z}_{s}\right)=0 .
$$

3. Equation for dynamics of the bar A:

$$
\rho \ddot{y}_{A}(x, t)+E I y_{A}^{\prime \prime \prime \prime}(x, t)=0 \text {. }
$$

4. Equation for dynamics of the bar B:

$$
\rho \ddot{y}_{B}(x, t)+E I y_{B}^{\prime \prime \prime \prime}(x, t)=0,
$$

where the boundary conditions are:

1. Bending moment:

$$
\left.\operatorname{EI} y_{A}^{\prime \prime}(x, t)\right|_{x=0} ^{x=L}=0 \quad,\left.\quad E I y_{B}^{\prime \prime}(x, t)\right|_{x=0} ^{x=L}=0
$$

2. Shear force for bar A:

- To $x=0$ :

$$
\begin{aligned}
E I y_{A}^{\prime \prime \prime}(0, t)= & k_{s}\left(z_{s}-\frac{l}{2} \sin \phi-y_{A}(0, t)\right)-k_{t}\left(y_{A}(0, t)-z_{r r}\right) \\
& +b_{s}\left(\dot{z}_{s}-\frac{l}{2} \dot{\phi} \cos \phi-\dot{y}_{A}(0, t)\right)-m_{u r} \ddot{y}_{A}(0, t)
\end{aligned}
$$

- To $x=L$ :

$$
E I y_{A}^{\prime \prime \prime}(L, t)=u
$$

3. Shear force for bar B:

- To $x=0$ :

$$
E I y_{B}^{\prime \prime \prime}(0, t)=u
$$


- To $x=L$ :

$$
\begin{aligned}
E I y_{B}^{\prime \prime \prime}(L, t)= & -k_{s}\left(z_{s}+\frac{l}{2} \sin \phi-y_{B}(L, t)\right)+k_{t}\left(y_{B}(L, t)-z_{r l}\right) \\
& -b_{s}\left(\dot{z}_{s}+\frac{l}{2} \dot{\phi} \cos \phi-\dot{y}_{B}(L, t)\right)+m_{u l} \ddot{y}_{B}(L, t) .
\end{aligned}
$$

\subsubsection{Solution}

Likewise the previous section, the FEM solution is employed, where:

$$
y_{k}(x, t)=\mathbf{N}_{k}(x) \mathbf{v}_{k}(t)
$$

and $k=A, B$ and the $\mathbf{N}_{k}(x)$ and $\mathbf{v}_{k}(t)$ has the same structure as in Equation 4.37. The other additional tools used for the FEM solution, as the assembly process and coordinate transformation is made equally according to the previous part. For the active system, the acceleration and curvature expressions are given by:

$$
\begin{aligned}
& \ddot{y}_{k}(x, t)=\mathbf{N}_{k}(x) \ddot{\mathbf{v}}_{k}(t), \\
& y_{k}^{\prime \prime}(x, t)=\mathbf{N}_{k}^{\prime \prime}(x) \mathbf{v}_{k}(t),
\end{aligned}
$$

where $k=A, B$ and $\mathbf{N}_{k}$ and $\mathbf{v}_{k}$ include all elements, after assembled.

Substituting the Equation 5.35 into Equation 5.24 leads to:

1. Equation for roll dynamics:

$$
\begin{aligned}
J_{s} \ddot{\phi} & -m_{s} g h \sin \phi+k_{s} \frac{l}{2} \cos \phi\left(\mathbf{N}_{\mathbf{A}}(0) \mathbf{v}_{\mathbf{A}}(t)-\mathbf{N}_{\mathbf{B}}(L) \mathbf{v}_{\mathbf{B}}(t)+l \sin \phi\right) \\
& +b_{s} \frac{l}{2} \cos \phi\left(\mathbf{N}_{\mathbf{A}}(0) \dot{\mathbf{v}}_{\mathbf{A}}(t)-\mathbf{N}_{\mathbf{B}}(L) \dot{\mathbf{v}}_{\mathbf{B}}(t)+l \dot{\phi} \cos \phi\right)-F_{l a t} h \cos \phi=0 .
\end{aligned}
$$

2. Equation for vertical translation dynamics of the sprung mass:

$$
\begin{aligned}
& m_{s} \ddot{z}_{s}+2 k_{s} z_{s}-k_{s}\left(\mathbf{N}_{\mathbf{A}}(0) \mathbf{v}_{\mathbf{A}}(t)+\mathbf{N}_{\mathbf{B}}(L) \mathbf{v}_{\mathbf{B}}(t)\right) \\
& -b_{s}\left(\mathbf{N}_{\mathbf{A}}(0) \dot{\mathbf{v}}_{\mathbf{A}}(t)+\mathbf{N}_{\mathbf{B}}(L) \dot{\mathbf{v}}_{\mathbf{B}}(t)-2 \dot{z}_{s}\right)=0 .
\end{aligned}
$$

3. Equation for dynamics of the bar A:

$$
\rho \mathbf{N}_{\mathbf{A}}(x) \ddot{\mathbf{v}}_{\mathbf{A}}(t)+E I \mathbf{N}_{\mathbf{A}}^{\prime \prime \prime \prime}(x) \mathbf{v}_{\mathbf{A}}(t)=0
$$

4. Equation for dynamics of the bar B:

$$
\rho \mathbf{N}_{\mathbf{B}}(x) \ddot{\mathbf{v}}_{\mathbf{B}}(t)+E I \mathbf{N}_{\mathbf{B}}^{\prime \prime \prime \prime}(x) \mathbf{v}_{\mathbf{B}}(t)=0
$$


where the resultants boundary conditions are:

1. Bending moment:

$$
\left.E I \mathbf{N}_{\mathbf{A}}^{\prime \prime}(x) \mathbf{v}_{\mathbf{A}}(t)\right|_{x=0} ^{x=L}=0 \quad,\left.\quad E I \mathbf{N}_{\mathbf{B}}^{\prime \prime}(x) \mathbf{v}_{\mathbf{B}}(t)\right|_{x=0} ^{x=L}=0
$$

2. Shear force for bar A:

- To $x=0$ :

$$
\begin{aligned}
& E I \mathbf{N}_{\mathbf{A}}^{\prime \prime \prime}(0) \mathbf{v}_{\mathbf{A}}(t)=k_{s}\left(z_{s}-\frac{l}{2} \sin \phi-\mathbf{N}_{\mathbf{A}}(0) \mathbf{v}_{\mathbf{A}}(t)\right)-k_{t}\left(\mathbf{N}_{\mathbf{A}}(0) \mathbf{v}_{\mathbf{A}}(t)-z_{r r}\right) \\
& +b_{s}\left(\dot{z}_{s}-\frac{l}{2} \dot{\phi} \cos \phi-\mathbf{N}_{\mathbf{A}}(0) \dot{\mathbf{v}}_{\mathbf{A}}(t)\right)-m_{u r} \mathbf{N}_{\mathbf{A}}(0) \ddot{\mathbf{v}}_{\mathbf{A}}(t)
\end{aligned}
$$

- To $x=L$ :

$$
E I \mathbf{N}_{\mathbf{A}}^{\prime \prime \prime}(L) \mathbf{v}(t)=u
$$

3. Shear force for bar B:

- To $x=0$ :

$$
E I \mathbf{N}_{\mathbf{B}}^{\prime \prime \prime}(0) \mathbf{v}(t)=u
$$

- To $x=L$ :

$$
\begin{aligned}
& E I \mathbf{N}_{\mathbf{B}}^{\prime \prime \prime}(L) \mathbf{v}_{\mathbf{B}}(t)=-k_{s}\left(z_{s}+\frac{l}{2} \sin \phi-\mathbf{N}_{\mathbf{B}}(L) \mathbf{v}_{\mathbf{B}}(t)\right)+k_{t}\left(\mathbf{N}_{\mathbf{B}}(L) \mathbf{v}_{\mathbf{B}}(t)-z_{r l}\right) \\
& -b_{s}\left(\dot{z}_{s}+\frac{l}{2} \dot{\phi} \cos \phi-\mathbf{N}_{\mathbf{B}}(L) \dot{\mathbf{v}}_{\mathbf{B}}(t)\right)+m_{u l} \mathbf{N}_{\mathbf{B}}(L) \ddot{\mathbf{v}}_{\mathbf{B}}(t) .
\end{aligned}
$$

In order to solve the Equation 5.38 and Equation 5.39, representing only the distributed portion, the weighted residuals method are applied as in the previous section:

$$
\int_{0}^{L} \omega(x)\left[\rho \mathbf{N}_{\mathbf{A}}(x) \ddot{\mathbf{v}}_{\mathbf{A}}(t)+E I \mathbf{N}_{\mathbf{A}}^{\prime \prime \prime \prime}(x) \mathbf{v}_{\mathbf{A}}(t)\right] \mathrm{d} x=0,
$$

and

$$
\int_{0}^{L} \omega(x)\left[\rho \mathbf{N}_{\mathbf{B}}(x) \ddot{\mathbf{v}}_{\mathbf{B}}(t)+E I \mathbf{N}_{\mathbf{B}}^{\prime \prime \prime \prime}(x) \mathbf{v}_{\mathbf{B}}(t)\right] \mathrm{d} x=0 .
$$

Applying the integration by parts, both in the terms with temporal derivative as in 
the terms with spatial derivative, one arrives in:

$$
\begin{aligned}
& \int_{0}^{L} \omega(x) \rho \mathbf{N}_{\mathbf{A}}(x) \mathrm{d} x \ddot{\mathbf{v}}_{\mathbf{A}}(t)+\int_{0}^{L} \omega^{\prime \prime}(x) E I \mathbf{N}_{\mathbf{A}}^{\prime \prime}(x) \mathrm{d} x \mathbf{v}_{\mathbf{A}}(t) \\
& -\left.\omega^{\prime}(x) E I \mathbf{N}_{\mathbf{A}}^{\prime \prime}(x)\right|_{x=0} ^{x=L} \mathbf{v}_{\mathbf{A}}(t)+\left.\omega(x) E I \mathbf{N}_{\mathbf{A}}^{\prime \prime \prime}(x)\right|_{x=0} ^{x=L} \mathbf{v}_{\mathbf{A}}(t)=0,
\end{aligned}
$$

and

$$
\begin{aligned}
& \int_{0}^{L} \omega(x) \rho \mathbf{N}_{\mathbf{B}}(x) \mathrm{d} x \ddot{\mathbf{v}}_{\mathbf{B}}(t)+\int_{0}^{L} \omega^{\prime \prime}(x) E I \mathbf{N}_{\mathbf{B}}^{\prime \prime}(x) \mathrm{d} x \mathbf{v}_{\mathbf{B}}(t) \\
& -\left.\omega^{\prime}(x) E I \mathbf{N}_{\mathbf{B}}^{\prime \prime}(x)\right|_{x=0} ^{x=L} \mathbf{v}_{\mathbf{B}}(t)+\left.\omega(x) E I \mathbf{N}_{\mathbf{B}}^{\prime \prime \prime}(x)\right|_{x=0} ^{x=L} \mathbf{v}_{\mathbf{B}}(t)=0 .
\end{aligned}
$$

By the Galerkin method the weight function is:

$$
\omega(x)=\frac{\partial y_{i}(x, t)}{\partial v_{i}(t)}=\mathbf{N}_{\mathbf{i}}^{T}(x)
$$

The residuals are then:

$$
M_{v_{A}} \ddot{\mathbf{v}}_{\mathbf{A}}(t)+K_{v_{A}} \mathbf{v}_{\mathbf{A}}(t)-\left.\mathbf{N}_{\mathbf{A}}^{\prime T}(x) E I \mathbf{N}_{\mathbf{A}}^{\prime \prime}(x)\right|_{x=0} ^{x=L} \mathbf{v}_{\mathbf{A}}(t)+\left.\mathbf{N}_{\mathbf{A}}^{T}(x) E I \mathbf{N}_{\mathbf{A}}^{\prime \prime \prime}(x)\right|_{x=0} ^{x=L} \mathbf{v}_{\mathbf{A}}(t)=0
$$

and

$$
M_{v_{B}} \ddot{\mathbf{v}}_{\mathbf{B}}(t)+K_{v_{B}} \mathbf{v}_{\mathbf{B}}(t)-\left.\mathbf{N}_{\mathbf{B}}^{\prime T}(x) E I \mathbf{N}_{\mathbf{B}}^{\prime \prime}(x)\right|_{x=0} ^{x=L} \mathbf{v}_{\mathbf{B}}(t)+\left.\mathbf{N}_{\mathbf{B}}^{T}(x) E I \mathbf{N}_{\mathbf{B}}^{\prime \prime \prime}(x)\right|_{x=0} ^{x=L} \mathbf{v}_{\mathbf{B}}(t)=0
$$

where $M_{v_{i}}$ and $K_{v_{i}}$ are the mass and stiffness matrices generated by the FEM described as in the following equations:

$$
\begin{aligned}
M_{v_{i}} & =\int_{0}^{L} \rho \mathbf{N}_{\mathbf{i}}^{T}(x) \mathbf{N}_{\mathbf{i}}(x) \mathrm{d} x, \\
K_{v_{i}} & =\int_{0}^{L} E I \mathbf{N}_{\mathbf{i}}^{\prime \prime T}(x) \mathbf{N}_{\mathbf{i}}^{\prime \prime}(x) \mathrm{d} x .
\end{aligned}
$$

The boundary conditions obtained in the active version are replaced into Equation 5.47 and Equation 5.48 to complete the hybridization between discrete and distributed portions, which yields:

$$
\begin{aligned}
& M_{v_{A}} \ddot{\mathbf{v}}_{\mathbf{A}}(t)+K_{v_{A}} \mathbf{v}_{\mathbf{A}}(t)+\mathbf{N}_{\mathbf{A}}^{T}(L) u-\mathbf{N}_{\mathbf{A}}^{\prime T}(0)\left[k_{s}\left(z_{s}-\frac{l}{2} \sin \phi-\mathbf{N}_{\mathbf{A}}(0) \mathbf{v}_{\mathbf{A}}(t)\right)\right. \\
& \left.+b_{s}\left(\dot{z}_{s}-\frac{l}{2} \dot{\phi} \cos \phi-\mathbf{N}_{\mathbf{A}}(0) \dot{\mathbf{v}}_{\mathbf{A}}(t)\right)-m_{u r} \mathbf{N}_{\mathbf{A}}(0) \ddot{\mathbf{v}}_{\mathbf{A}}(t)-k_{t}\left(\mathbf{N}_{\mathbf{A}}(0) \mathbf{v}_{\mathbf{A}}(t)-z_{r r}\right)\right]=0
\end{aligned}
$$


and

$$
\begin{aligned}
& M_{v_{B}} \ddot{\mathbf{v}}_{\mathbf{B}}(t)+K_{v_{B}} \mathbf{v}_{\mathbf{B}}(t)-\mathbf{N}_{\mathbf{B}}^{T}(0) u-\mathbf{N}_{\mathbf{B}}^{\prime T}(L)\left[k_{s}\left(z_{s}+\frac{l}{2} \sin \phi-\mathbf{N}_{\mathbf{B}}(L) \mathbf{v}_{\mathbf{B}}(t)\right)\right. \\
& \left.b_{s}\left(\dot{z}_{s}+\frac{l}{2} \dot{\phi} \cos \phi-\mathbf{N}_{\mathbf{B}}(L) \dot{\mathbf{v}}_{\mathbf{B}}(t)\right)-m_{u l} \mathbf{N}_{\mathbf{B}}(L) \ddot{\mathbf{v}}_{\mathbf{B}}(t)-k_{t}\left(\mathbf{N}_{\mathbf{B}}(L) \mathbf{v}_{\mathbf{B}}(t)-z_{r l}\right)\right] .
\end{aligned}
$$

The equations obtained are non-linear and not suitable for linear control design. A linearization will be done by considering small angles, where it is assumed that $\cos \phi \approx 1$ and $\sin \phi \approx \phi$. Afterthat, the Equation 5.36, Equation 5.37, Equation 5.53 and Equation 5.54 can be arranged in the classic mechanical matricial form:

$$
\begin{aligned}
& {\left[\begin{array}{cccc}
J_{s} & 0 & 0 & 0 \\
0 & m_{s} & 0 & 0 \\
0 & 0 & m_{22} & 0 \\
0 & 0 & 0 & m_{33}
\end{array}\right]\left[\begin{array}{c}
\ddot{\phi} \\
\ddot{z}_{s} \\
\ddot{\mathbf{v}}_{\mathbf{A}} \\
\ddot{\mathbf{v}}_{\mathbf{B}}
\end{array}\right]+} \\
& \begin{array}{r}
{\left[\begin{array}{cccc}
b_{s} \frac{l^{2}}{2} & 0 & b_{s} \frac{l}{2} \mathbf{N}_{\mathbf{A}}(0) & -b_{s} \frac{l}{2} \mathbf{N}_{\mathbf{B}}(L) \\
0 & 2 b_{s} & -b_{s} \mathbf{N}_{\mathbf{A}}(0) & -b_{s} \mathbf{N}_{\mathbf{B}}(L) \\
b_{s} \frac{l}{2} \mathbf{N}_{\mathbf{A}}^{T}(0) & -b_{s} \mathbf{N}_{\mathbf{A}}^{T}(0) & b_{s} \mathbf{N}_{\mathbf{A}}^{T}(0) \mathbf{N}_{\mathbf{A}}(0) & 0 \\
-b_{s} \frac{l}{2} \mathbf{N}_{\mathbf{B}}^{T}(L) & -b_{s} \mathbf{N}_{\mathbf{B}}^{T}(L) & 0 & b_{s} \mathbf{N}_{\mathbf{B}}^{T}(L) \mathbf{N}_{\mathbf{B}}(L)
\end{array}\right]\left[\begin{array}{c}
\dot{\phi} \\
\dot{z}_{s} \\
\dot{\mathbf{v}}_{\mathbf{A}} \\
\dot{\mathbf{v}}_{\mathbf{B}}
\end{array}\right]+} \\
{\left[\begin{array}{cccc}
k_{s} \frac{l^{2}}{2}-m_{s} g h & 0 & k_{s} \frac{l}{2} \mathbf{N}_{\mathbf{A}}(0) & -k_{s} \frac{l}{2} \mathbf{N}_{\mathbf{B}}(L) \\
0 & 2 k_{s} & -k_{s} \mathbf{N}_{\mathbf{A}}(0) & -k_{s} \mathbf{N}_{\mathbf{B}}(L) \\
k_{s} \frac{l}{2} \mathbf{N}_{\mathbf{A}}^{T}(0) & -k_{s} \mathbf{N}_{\mathbf{A}}^{T}(L) & k_{22} & 0 \\
-k_{s} \frac{l}{2} \mathbf{N}_{\mathbf{B}}^{T}(L) & -k_{s} \mathbf{N}_{\mathbf{B}}^{T}(L) & 0 & k_{33}
\end{array}\right]\left[\begin{array}{c}
\phi \\
z_{s} \\
\mathbf{v}_{\mathbf{A}} \\
\mathbf{v}_{\mathbf{B}}
\end{array}\right]}
\end{array} \\
& {\left[\begin{array}{c}
0 \\
0 \\
-\mathbf{N}_{\mathbf{A}}^{T}(L) \\
\mathbf{N}_{\mathbf{B}}^{T}(0)
\end{array}\right] u+\left[\begin{array}{ccc}
m_{s} h & 0 & 0 \\
0 & 0 & 0 \\
0 & k_{t} \mathbf{N}_{\mathbf{A}}^{T}(0) & 0 \\
0 & 0 & k_{t} \mathbf{N}_{\mathbf{B}}^{T}(L)
\end{array}\right]\left[\begin{array}{c}
a_{y} \\
z_{r r} \\
z_{r l}
\end{array}\right]}
\end{aligned}
$$

where:

$$
\begin{aligned}
& m_{22}=M_{v_{A}}+m_{u r} \mathbf{N}_{\mathbf{A}}^{T}(0) \mathbf{N}_{\mathbf{A}}(0) \\
& m_{33}=M_{v_{B}}+m_{u l} \mathbf{N}_{\mathbf{B}}^{T}(L) \mathbf{N}_{\mathbf{B}}(L) \\
& k_{22}=K_{v_{A}}+\left(k_{s}+k_{t}\right) \mathbf{N}_{\mathbf{A}}^{T}(0) \mathbf{N}_{\mathbf{A}}(0) \\
& k_{33}=K_{v_{B}}+\left(k_{s}+k_{t}\right) \mathbf{N}_{\mathbf{B}}^{T}(L) \mathbf{N}_{\mathbf{B}}(L) \\
& M_{v i}=\int_{0}^{L} \rho \mathbf{N}_{\mathbf{i}}^{T}(x) \mathbf{N}_{\mathbf{i}}(x) \mathrm{d} x, \quad i=A, B \\
& K_{v i}=\int_{0}^{L} E I \mathbf{N}_{\mathbf{i}}{ }^{\prime \prime T}(x) \mathbf{N}_{\mathbf{i}}{ }^{\prime \prime}(x) \mathrm{d} x, \quad i=A, B
\end{aligned}
$$


The Equation 5.55 is, then, linear and will be used later in the control design, which is the convenient representation for linear control methods, as the LQG/LTR and $H_{\infty}$ designs.

\subsection{Lateral acceleration as a disturbance}

In this work, the influence of the road inputs, $z_{r l}$ and $z_{r r}$, will not be taken into account. Then, the lateral acceleration, $a_{y}$, will be considered as the only disturbance acting on the vehicle. This entry is not known previously as it will be determined from a maneuver. Therefore, the relation between the handwheel and the lateral acceleration can be built through a dynamic system. The modeling of this system is omitted and for more information about how the equations are derived, see (ABE, 2015) and (RAJAMANI, 2012). The dynamic system was implemented by the following equations:

$$
\begin{gathered}
\ddot{\delta}=-\ddot{\psi}-\frac{C_{s}}{I_{w}} \dot{\delta}+\frac{2 \epsilon C_{f}}{I_{w}}\left(\arctan \left(\beta+\frac{l_{f} \dot{\psi}}{V_{x}}\right)-\delta\right)+\frac{K_{s}}{I_{w}}\left(\frac{\alpha}{N_{G}}-\delta\right), \\
\dot{\beta}=-\dot{\psi}+\frac{C_{f}}{m V_{x}}\left(\delta-\arctan \left(\beta+\frac{l_{f} \dot{\psi}}{V_{x}}\right)\right)+\frac{C_{r}}{m V_{x}}\left(-\arctan \left(\beta-\frac{l_{r} \dot{\psi}}{V_{x}}\right)\right), \\
\ddot{\psi}=\frac{l_{f} C_{f}}{I_{z}}\left(\delta-\arctan \left(\beta+\frac{l_{f} \dot{\psi}}{V_{x}}\right)\right)-\frac{l_{r} C_{r}}{I_{z}}\left(-\arctan \left(\beta-\frac{l_{r} \dot{\psi}}{V_{x}}\right)\right), \\
a_{y}=V_{x}(\dot{\beta}+\dot{\psi}) .
\end{gathered}
$$

The parameters of the disturbance model described above are listed in Table 3 . 
Table 3: Designation of the parameters.

\begin{tabular}{|c||l|}
\hline Symbol & Description \\
\hline$\dot{\psi}$ & yaw rate \\
$\delta$ & side-slip angle \\
$\alpha$ & wheel angle \\
$m$ & handwheel angle \\
$l_{f}$ & total vehicle mass \\
$l_{r}$ & distance from c.g. to the front wheels \\
$C_{f}$ & distance from c.g. to the rear wheels \\
$C_{r}$ & front tire lateral stiffness \\
$I_{w}$ & rear tire lateral stiffness \\
$I_{z}$ & Inertia of the two front wheels \\
$V_{x}$ & yaw moment of inertia about c.g. \\
$N_{G}$ & longitudinal velocity \\
$K_{s}$ & steering ratio \\
$C_{s}$ & steering spring stiffness \\
$\epsilon$ & steering viscous damping ratio \\
\hline
\end{tabular}

Source: Author.

\subsection{Bar geometry for the FEM}

The Figure 27 illustrates the spatial discretization of the active bar. The Tables 4 and 5 show the global coordinates $\mathrm{x}-\mathrm{y}-\mathrm{z}$ of each node:

Figure 27: Geometry of the active anti-roll bar.

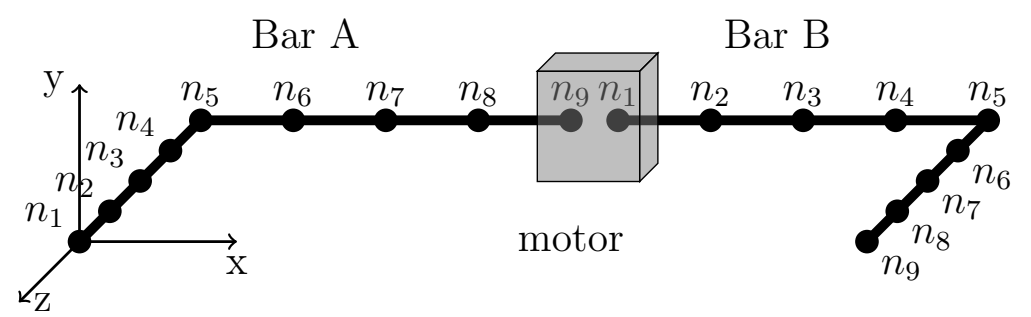

Source: Author. 
Table 4: Geometric coordinates of active bar A.

\begin{tabular}{|c|c|c|c|}
\hline \multicolumn{3}{|c|}{ Spatial distribution of the nodes } \\
\hline Description & $\mathrm{x}$ & $\mathrm{y}$ & $\mathrm{z}$ \\
\hline$n_{1}$ & 0 & 0 & 0 \\
$n_{2}$ & 0 & 0 & -0.025 \\
$n_{3}$ & 0 & 0 & -0.050 \\
$n_{4}$ & 0 & 0 & -0.075 \\
$n_{5}$ & 0 & 0 & -0.1 \\
$n_{6}$ & 0.15 & 0 & -0.1 \\
$n_{7}$ & 0.30 & 0 & -0.1 \\
$n_{8}$ & 0.45 & 0 & -0.1 \\
$n_{9}$ & 0.60 & 0 & -0.1 \\
\hline
\end{tabular}

Source: Author.

Table 5: Geometric coordinates of active bar B.

\begin{tabular}{|c||c|c|c|}
\hline \multicolumn{3}{|c|}{ Spatial distribution of the nodes } \\
\hline Description & $\mathrm{x}$ & $\mathrm{y}$ & $\mathrm{z}$ \\
\hline$n_{1}$ & 0.60 & 0 & -0.1 \\
$n_{2}$ & 0.75 & 0 & -0.1 \\
$n_{3}$ & 0.90 & 0 & -0.1 \\
$n_{4}$ & 1.05 & 0 & -0.1 \\
$n_{5}$ & 1.20 & 0 & -0.1 \\
$n_{6}$ & 1.20 & 0 & -0.075 \\
$n_{7}$ & 1.20 & 0 & -0.050 \\
$n_{8}$ & 1.20 & 0 & -0.025 \\
$n_{9}$ & 1.20 & 0 & 0 \\
\hline
\end{tabular}

Source: Author.

\subsection{Parameters}

The cross-section of the bar for both passive and active systems is circular, as shown in Figure 28: 
Figure 28: Bar cross-section.

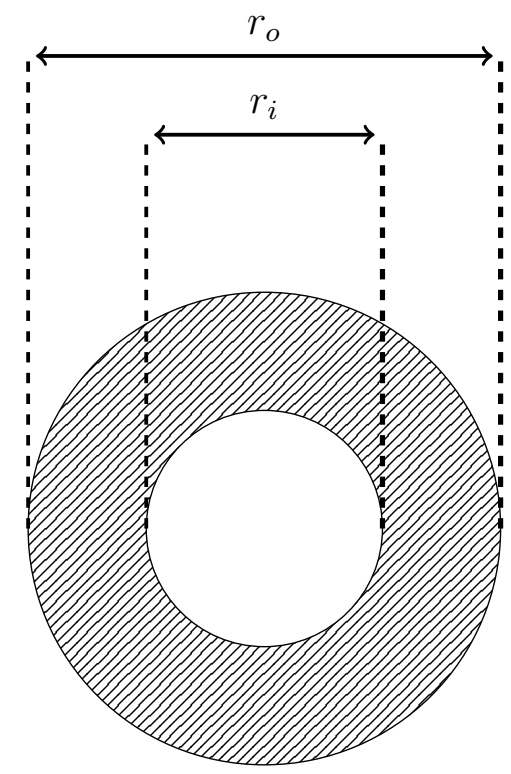

Source: Author.

where $r_{i}$ and $r_{o}$ are given in Table 6. The Table 6 presents the parameter values used for simulation. These parameters were taken from a car in software ADAMS/Car@.

Table 6: Model's parameters values.

\begin{tabular}{|c|c|c|}
\hline Variable & Value & Units \\
\hline$m_{s}$ & 950 & $\mathrm{~kg}$ \\
$m_{u r}$ & 47.35 & $\mathrm{~kg}$ \\
$m_{u l}$ & 47.35 & $\mathrm{~kg}$ \\
$h$ & 0.45 & $\mathrm{~m}$ \\
$I_{x x}$ & 298.62 & $\mathrm{kgm}$ \\
$k_{s}$ & 95000 & $\mathrm{~N} / \mathrm{m}$ \\
$b_{s}$ & 10262.6 & $\mathrm{n} / \mathrm{m} / \mathrm{s})$ \\
$k_{t}$ & 310000 & $\mathrm{~N} / \mathrm{m}$ \\
$l$ & 1.2 & $\mathrm{~m}$ \\
$g$ & 9.81 & $\mathrm{~m} / \mathrm{s}^{2}$ \\
$E$ & 210 & $\mathrm{GPa}$ \\
$\rho$ & 7850 & $\mathrm{~kg} / \mathrm{m}^{3}$ \\
$r_{o}$ & 21.8 & $\mathrm{~mm}$ \\
$r_{i}$ & 16.0 & $\mathrm{~mm}$ \\
\hline
\end{tabular}

Source: ADAMS/Carß). 


\subsection{Validation}

The vehicle's parameters used in this study is from Adams software and the author's model is validated by comparison with the respective Adams simulation. The Adams's vehicle is constituted by subsystems and test rig for a full vehicle, which consists of front/rear suspensions, steering subsystem, powertrain, brake subsystem, front/rear tires and rigid chassis.

In order to compare both models, the vehicle will be subjected to a well known maneuver, which is the lane change. The Figure 29 shows the lane change input and the vehicle response corresponding to that input, both the Adams simulation and the author's model with the Adams' parameters substituted.

Figure 29: Response comparison of the author's model and ADAMS/Carß: (a) steering input, (b) side-slip angle, (c) yaw rate, and (d) lateral acceleration.

(a)

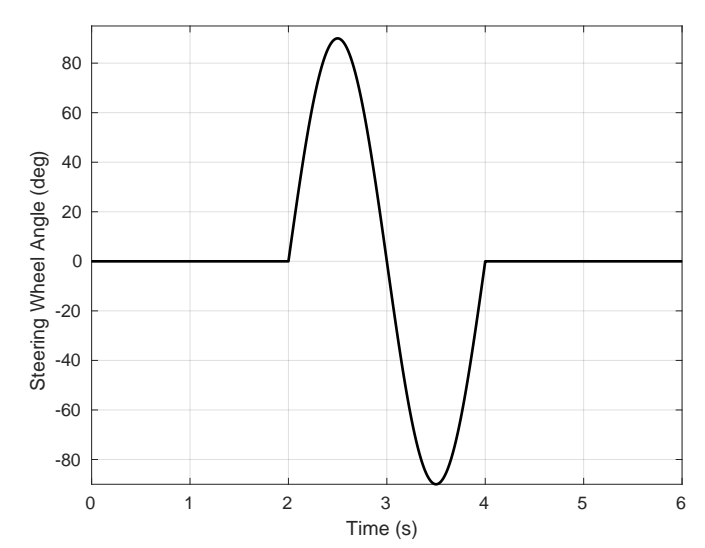

(c)

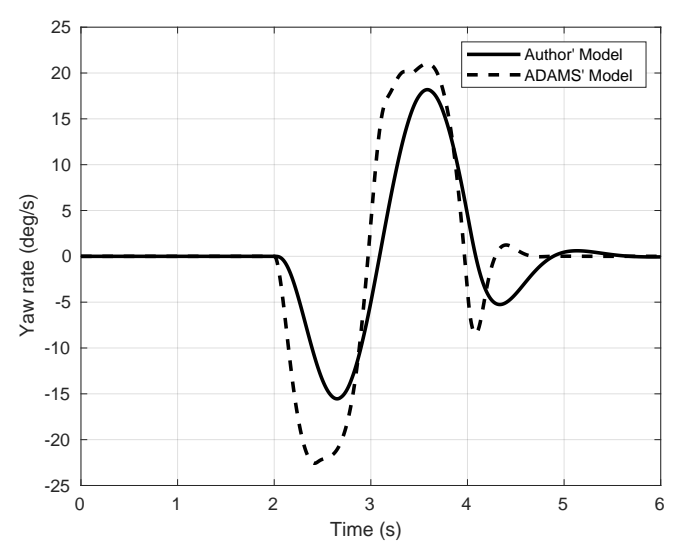

(b)

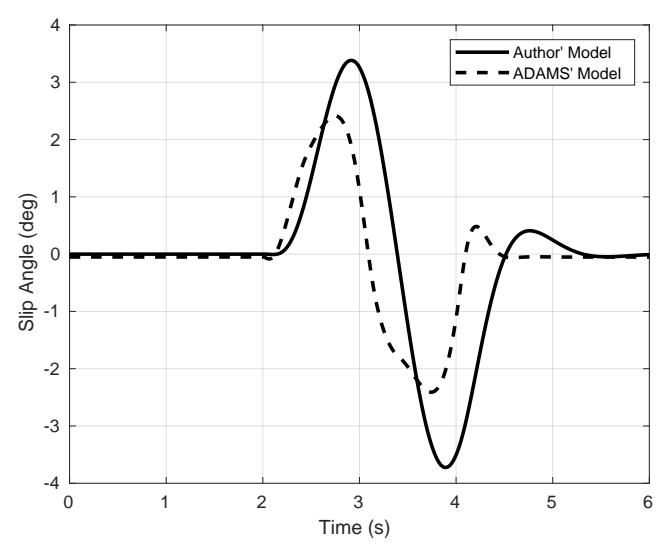

(d)

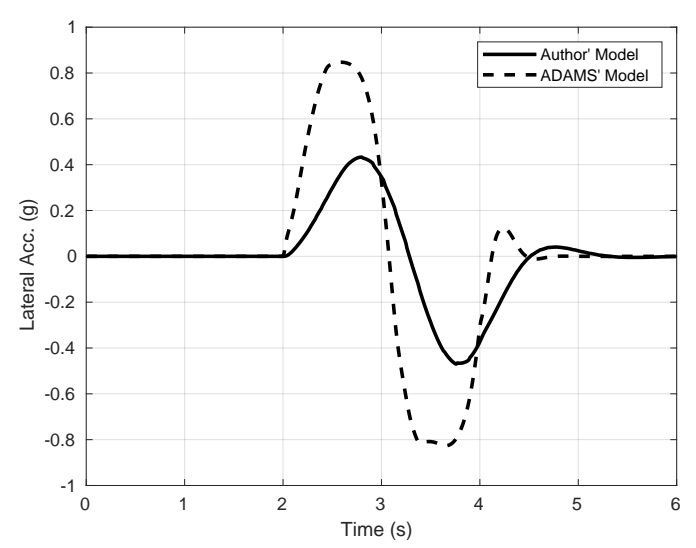

Source: Author. 
The Adams's model implements a full vehicle, considering complex modeling as tires, complete steering system, where all the systems are coupled. Considering the simplicity of the developed vehicle, that must be simple enough to be useful in a control design, it can be concluded that the author's model is satisfactory since the response of this model follows in general the behavior of the response generated by Adams. Therefore, the vehicle model of this study is concluded to be acceptable and useful for the purpose of this work.

Next chapter will present the main concepts of the robustness analysis used in this work and the control theory to design the LQG/LTR and $H_{\infty}$ loop-shaping controllers. 


\section{CONTROL DESIGN}

This chapter is intended to present the main concepts used to design a robust control system. Among the related subjects, it includes uncertainty models, which are used when designing a robust procedure, and also includes a SISO system analysis of robustness based on frequency responses. In addition, the two methods of robust controllers, LQG/LTR and $H_{\infty}$, are discussed and, posteriorly, used in the active anti-roll system.

\subsection{Representations of uncertainty}

Uncertainties are unavoidable when a real system is mathematically modeled. It can be sorted in two main categories: signals disturbances and dynamics perturbations. The first category includes input/output disturbances, as sensor/actuator noises, etc. The second one is related to discrepancies among mathematical models and the real operation by the system dynamics. Any mathematical model of a system is always an approximation of the real system and of its physical behavior. Typical sources of these differences are non-modeled dynamics, normally in high frequencies, neglected non-linearities, effects on order reduction of systems, parameters variation with environment conditions, among others (GU; PETKOV; KONSTANTINOV, 2005).

In robust control theory, it is important to find a mathematical representation of the model uncertainty in order to be possible to determine whether the system remains stable for all plants, and then, determine whether the performance specifications are met for all plants in the uncertainty set (SKOGESTAD; POSTLETHWAITE, 2007). Let us see the uncertainty models relative to this work.

1. Additive perturbation

$$
G_{\Delta}(s)=G(s)+\Delta(s)
$$


Figure 30: Additive perturbation.

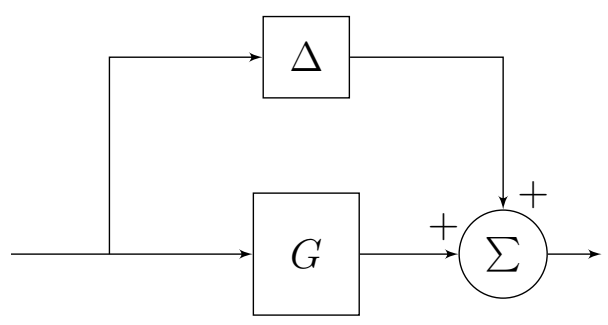

Source: Author.

2. Multiplicative perturbation

$$
G_{\Delta}(s)=(1+\Delta(s)) G(s) .
$$

Figure 31: Multiplicative perturbation.

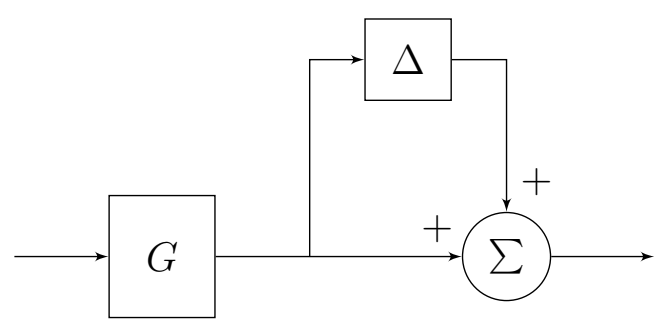

Source: Author.

3. Left coprime factor perturbations

$$
G_{\Delta}(s)=\left(M+\Delta_{M}\right)^{-1}\left(N+\Delta_{N}\right)
$$

Figure 32: Left coprime factor perturbations.

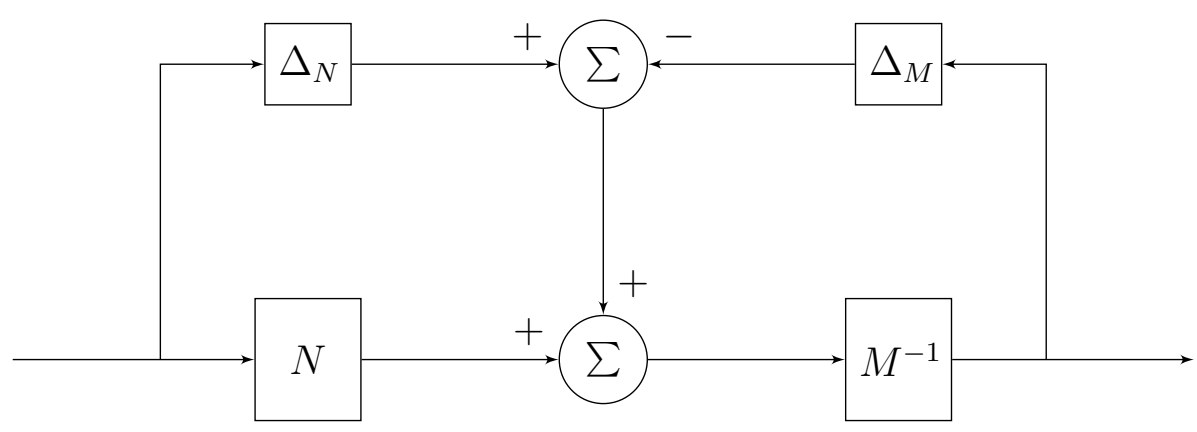

Source: Author.

Notice that the additive perturbations leads to values of absolute errors between the nominal $G(s)$ and real $G_{\Delta}(s)$ systems. On the other hand, the multiplicative perturbations 
considers relative errors. The choice of one of these options must be done caring the treatment of the errors, once it influences the robustness analysis.

The block $\Delta$ is unknown, therefore, it is the source of the uncertainty. However, it is normally limited by a known function, as $\bar{\sigma}(\Delta(j \omega)) \leq \delta(j \omega)$, for all frequencies $\omega$, where $\delta$ is a known scalar function and $\bar{\sigma}($.$) is the largest singular value of a matrix (\mathrm{GU}$; PETKOV; KONSTANTINOV, 2005). The representations using $M$ and $N$ are known as coprime factorization of the nominal system $G$. The $\Delta_{M}$ and $\Delta_{N}$ are the perturbations on the corresponding factors.

\subsection{Performance and robustness of feedback systems}

The effects of uncertainty are reduced by using feedback and, in addition, feedback is used to stabilize an unstable system. A feedback system deals with unwanted or unexpected signals and the assessment of how it behaves in face of such signals is known as performance. The robustness is defined from the assessment of how resilient it is in face of behavior internal changes (e.g. unmodeled dynamics) (MACIEJOWSKI, 1989). In this section is presented the concepts related to the SISO systems, which stand for Single-Input-Single-Output.

Figure 33 shows the typical block diagram of a feedback problem. The real plant is represented by the transfer function $G_{\Delta(s)}$, which can assume one of the forms presented in previous section of uncertainty, the disturbance signal $d(s)$, and the input and output signals $u(s)$ and $y(s)$, respectively. The reference signal is represented by the function $r(s)$, which is to be followed by the plant output $y(s)$, and $n(s)$ represents measurements errors. $K(s)$ is called the feedback compensator, also known as controller, and represents the dynamic system which are to be designed.

Figure 33: General block diagram of a feedback system.

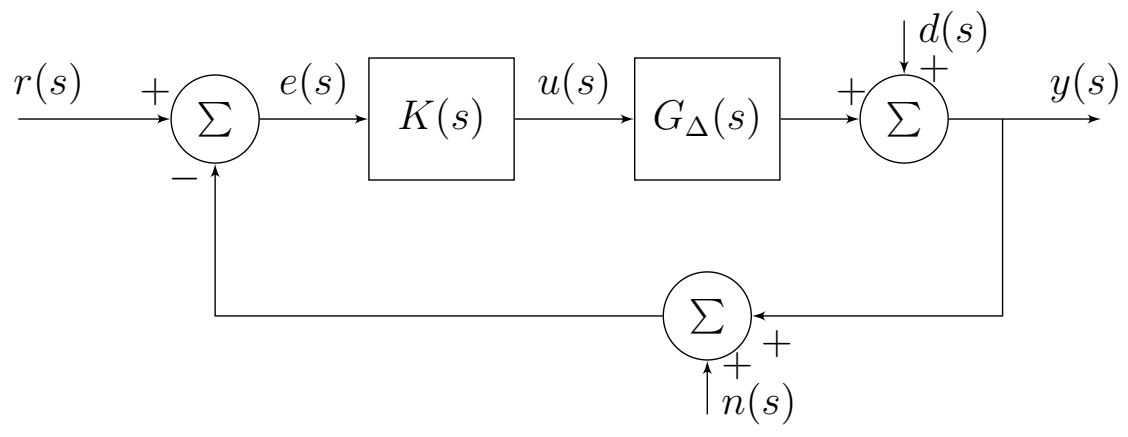

Source: Author. 
From the system represented in Figure 33, the following equations can be derived:

$$
\begin{aligned}
& y(s)=\underbrace{\frac{G(s) K(s)}{1+G(s) K(s)}}_{T(s)} r(s)+\underbrace{\frac{1}{1+G(s) K(s)}}_{S(s)} d(s)-\frac{G(s) K(s)}{1+G(s) K(s)} n(s), \\
& e(s)=\frac{1}{1+G(s) K(s)} r(s)-\frac{1}{1+G(s) K(s)} d(s)-\frac{1}{1+G(s) K(s)} n(s) .
\end{aligned}
$$

The Equation 6.4 shows the sensitivity function $S(s)$, that relates the disturbance $d(s)$ with the output $y(s)$, and the complementary sensitivity function $T(s)$, that relates the input $r(s)$ with the output $y(s)$, which is the closed-loop transfer function. Then:

$$
\begin{aligned}
& T(s)=\frac{G(s) K(s)}{1+G(s) K(s)}, \\
& S(s)=\frac{1}{1+G(s) K(s)},
\end{aligned}
$$

where:

$$
T(s)+S(s)=1
$$

\subsection{The nominal case}

In the next analysis, it is supposed the stability of the nominal plant $G(s)$, and, from now on, it will be used the notation $s=j \omega$ for representing the analysis of the frequency portion on complex variable $s$.

\subsubsection{Tracking of reference signal}

Consider the relation between $r(j \omega)$ and $e(j \omega)$ in Equation 6.5.

$$
e(j \omega)=\frac{1}{1+G(j \omega) K(j \omega)} r(j \omega)
$$

and $\Omega_{r}$ is known as the region where $r(j \omega)$ has the more relevant part of the energy. Then, $\Omega_{r}=\left\{\omega: \omega \leq \omega_{r}\right\}$, with $\omega_{r}$ given. As part of the project, it is admitted that $\alpha_{r}(\omega), \omega \in \Omega_{r}$, is a function so that:

$$
\frac{|e(j \omega)|}{|r(j \omega)|} \leq \alpha_{r}(\omega), \quad \omega \in \Omega_{r}
$$


Typically, the function $\alpha_{r}(\omega)$ is made constant and much smaller than one. As a consequence, we take approximately:

$$
|G(j \omega) K(j \omega)| \geq \frac{1}{\alpha_{r}(\omega)}, \quad \omega \in \Omega_{r}
$$

Therefore, considering the gain of the open-loop transfer function as large as possible in the region $\Omega_{r}$, yields that $y(j \omega) \approx r(j \omega)$.

\subsubsection{Disturbance rejection}

Consider now the relation between $d(j \omega)$ and $e(j \omega)$ :

$$
e(j \omega)=-\frac{1}{1+G(j \omega) K(j \omega)} d(j \omega)
$$

and $\Omega_{d}$ being the region where $d(j \omega)$ has the more relevant part of the energy. Then, $\Omega_{d}=\left\{\omega: \omega \leq \omega_{d}\right\}$, with $\omega_{d}$ given. As a given specification, a function $\alpha_{d}(\omega), \omega \in \Omega_{d}$, is given as:

$$
\frac{|e(j \omega)|}{|d(j \omega)|} \leq \alpha_{d}(\omega), \quad \omega \in \Omega_{d}
$$

As in the case of reference tracking, the function $\alpha_{d}(\omega)$ is also made constant and much smaller than one. Then, as result of this assumption:

$$
|G(j \omega) K(j \omega)| \geq \frac{1}{\alpha_{d}(\omega)}, \quad \omega \in \Omega_{d}
$$

Hence, in order to diminish the influence of disturbance and tracking error, the openloop gain must be as larger as possible in the union of $\Omega_{r}$ and $\Omega_{d}$.

\subsubsection{Measurement error rejection}

From Equation 6.4, the relation between $n(j \omega)$ and $y(j \omega)$ is given as:

$$
y(j \omega)=-\frac{G(j \omega) K(j \omega)}{1+G(j \omega) K(j \omega)} n(j \omega)
$$

where $\Omega_{n}$ is the region where $n(j \omega)$ has the larger part of the energy. In general, $\Omega_{n}=$ $\left\{\omega: \omega \geq \omega_{n}\right\}$ and $\omega_{n}$ is set by the designer according to the frequency response of the source of noise. A function $\alpha_{n}(\omega), \omega \in \Omega_{n}$, can be defined where:

$$
\frac{|y(j \omega)|}{|n(j \omega)|} \leq \alpha_{n}(\omega), \quad \omega \in \Omega_{n}
$$


Typically, the function $\alpha_{n}(\omega)$ is constant and smaller than one. In order to obtain a condition which ensures the measurement error according to the specified margin, it is necessary that:

$$
|G(j \omega) K(j \omega)| \ll 1
$$

which implies that:

$$
|1+G(j \omega) K(j \omega)| \approx 1
$$

and, finally, it is possible to obtain the approximated condition:

$$
|G(j \omega) K(j \omega)| \leq \alpha_{n}(j \omega) \ll 1, \quad \omega \in \Omega_{n}
$$

Therefore, to prevent the influence of measure errors at the output, the gain of the open-loop transfer function must be as small as possible over the frequency range where the measurement error is large. The Figure 34 summarizes the results obtained.

Figure 34: Barriers over the frequency range.

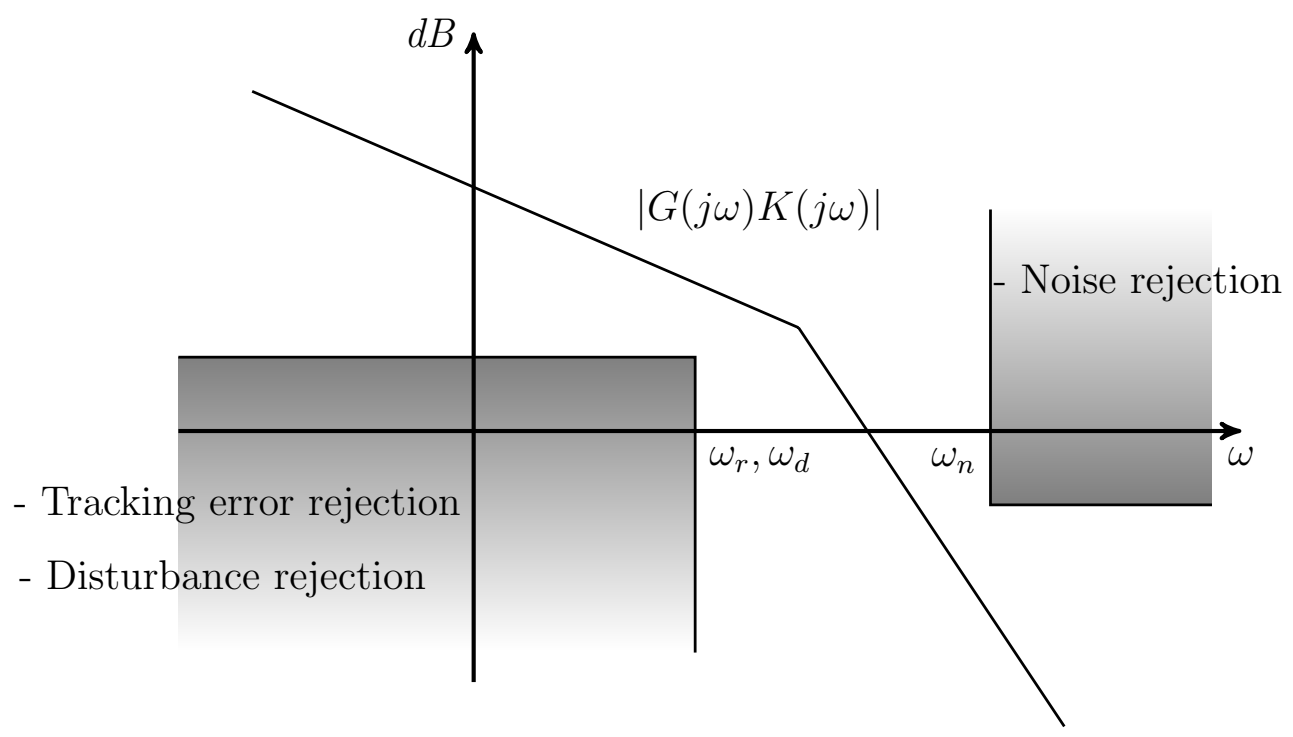

Source: Author.

\subsection{The real case}

Let's consider the multiplicative perturbation, as in Figure 31, in order to analyze the real system, which is treated as $G_{\Delta}(s)$. By this choice, it is used the multiplicative error $\varepsilon_{M}(s)$, defined by:

$$
\varepsilon_{M}(s)=\frac{G_{\Delta}(s)-G(s)}{G(s)}
$$


which is a relative measure of the error (CRUZ, 1996). It is assumed that the control designer is able to establish a function as an upper limit to $\left|\varepsilon_{M}(j \omega)\right|$, so that:

$$
\left|\varepsilon_{M}(j \omega)\right| \leq e_{M}(\omega), \quad \forall \omega \in \mathbb{R}
$$

\subsubsection{Robust stability}

The fundamental task, at first, is to ensure the stability of the real (i.e. uncertain) system. This means the stability of the closed-loop transfer function of the real system, defined as $T_{\Delta}(s)$ :

$$
T_{\Delta}(s)=\frac{G_{\Delta} K(s)}{1+G_{\Delta} K(s)},
$$

where the Equation 6.20 must be guaranteed.

Using the Nyquist theorem for stability (see (MACIEJOWSKI, 1989)), is possible to write the stability condition to the real system. This theorem states that: if $G_{\Delta}(s)$ has $N$ unstable poles, then the closed-loop system $T_{\Delta}(s)$ is stable if and only if the characteristic loci of the open-loop transfer function $G_{\Delta}(s) K(s)$ encircle the point $-1+j 0$ in $N$ times anticlockwise, assuming that there are no hidden unstable modes (MACIEJOWSKI, 1989). Using the Equation 6.19 and $s=j \omega$, a condition that there is no crossing at point $-1+j 0$ can be written as:

$$
\left(1+\varepsilon_{M}(j \omega)\right) G(j \omega) K(j \omega) \neq-1+j 0,
$$

where $\varepsilon_{M}(j \omega)$ satisfy Equation 6.20.

Similarly, the Equation 6.22 can be rewritten as:

$$
1+\varepsilon_{M}(j \omega) T(j \omega) \neq 0
$$

which is assumed that $T(s)$ is asymptotically stable, and, then, $1+G(j \omega) K(j \omega) \neq 0$ (CRUZ, 1996). Considering the relation between $\varepsilon_{M}(j \omega)$ and $e_{M}(\omega)$ (Equation 6.20), it is established that:

$$
|T(j \omega)|<\frac{1}{e_{M}(\omega)},
$$

which is the robustness stability condition. 


\subsubsection{Performance}

In the same way as in nominal case, the general performance condition can be written as:

$$
\left|G_{\Delta}(j \omega) K(j \omega)\right| \geq p(\omega), \quad \forall \omega \leq \omega_{0},
$$

where $\omega_{0}=\max \left[\omega_{r}, \omega_{d}\right]$. From Equation 6.19, the condition above can be described in terms of the nominal system as:

$$
|G(j \omega) K(j \omega)|\left|1+\varepsilon_{M}(j \omega)\right| \geq p(\omega), \quad \forall \omega \leq \omega_{0} .
$$

At this point, is necessary to consider the frequency region of the performance characteristic. The reference input and disturbance signal, normally, has its energy concentrated at low frequencies, where it is supposed that $e_{M}(\omega)<1$. Based on this assumption and Equation 6.20, the condition (Equation 6.26) can be rewritten as:

$$
|G(j \omega) K(j \omega)| \geq \frac{p(\omega)}{1-e_{M}(\omega)}, \quad \forall \omega \leq \omega_{0}
$$

Equation 6.27 will be considered the robustness performance condition. The Figure 35 summarizes the results presented.

Figure 35: Barriers under influence of $e_{M}(\omega)$.

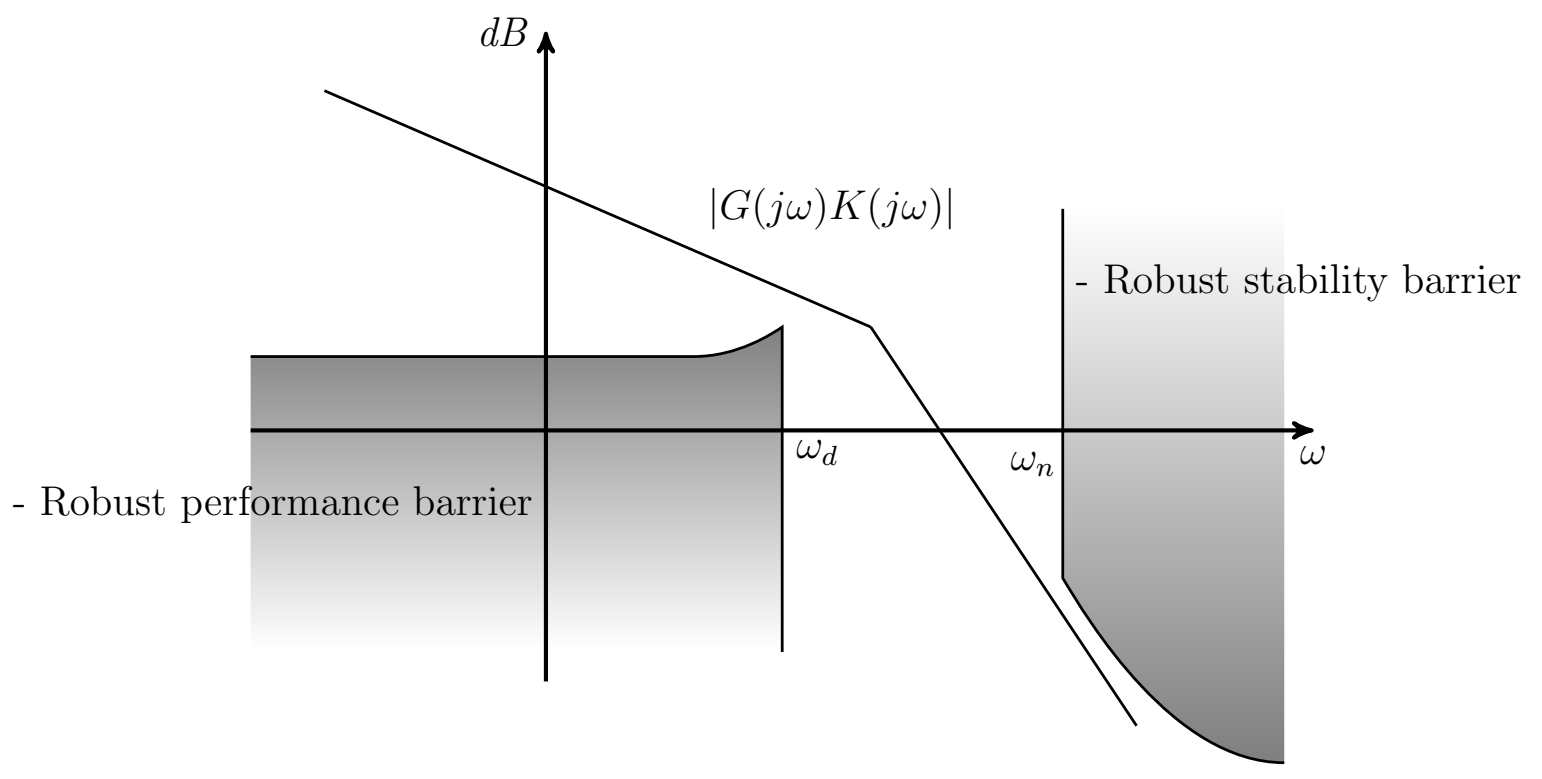

Source: Author. 


\subsection{LQG/LTR control method}

In this section, it is presented a design method based on optimal control theory, called Linear Quadratic Gaussian or LQG design method. After stated the main results of this theory, a procedure to synthesize the optimal state-feedback compensator is present, known as Loop Transfer Recovery or LTR procedure. For more details, see (ANDERSON; MOORE; MOLINARI, 1972) and (DOYLE, 1978). The process of designing the control law using Linear Quadratic Gaussian (LQG) controller is performed in two steps for the linearized active anti-roll system model, expressed in following form:

$$
\begin{aligned}
& \dot{x}=A x+B u+G w, \\
& y=C x+v
\end{aligned}
$$

where $w$ and $v$ are, respectively, the process and the measurement Gaussian white noise.

Figure 36: LQG scheme blocks.

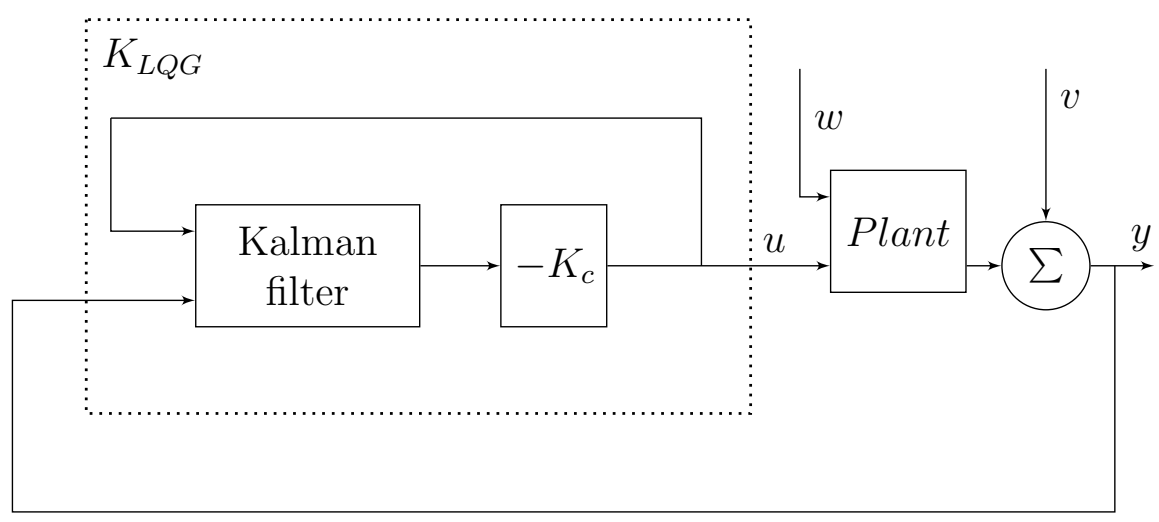

Source: Author.

Taking into account Figure 37, the Kalman filter is firstly determined by minimizing the steady-state error covariance $E\left[e_{0}(t), e_{0}^{T}(t)\right]$ where $e_{0}(t)=x(t)-x_{0}(t)$. The process of minimizing the error covariance is strongly associated with choice of weighting matrices $W$ and $V$ presented in Equation 6.29, which each of them represents covariance of process and measurement noise, respectively:

$$
E\left[w w^{T}\right]=W, \quad E\left[v v^{T}\right]=V, \quad E\left[w v^{T}\right]=N
$$

\subsubsection{Quadratic cost function}

In the LQG method, the quadratic term refers to the performance index that is in the controller synthesis. In Equation 6.28, $u$ represents the vector of control signals and $y$ the 
vector of measured outputs corrupted by noise $v$. If the system is SISO, $u$ and $y$ will be scalar. The linear quadratic regulator (LQR) minimizes the quadratic performance index given by:

$$
J_{L Q R}=\int_{0}^{\infty}\left[\boldsymbol{z}^{T}(t) Q \boldsymbol{z}(t)+\boldsymbol{u}^{T}(t) R \boldsymbol{u}(t)\right] d t
$$

where $z=M x$ for some linear combinations of states. In most cases $M$ is considered to be the output matrix $C$ of the plant model. The LQG controller has as its performance criterion the covariance of the quadratic performance index, which is:

$$
\begin{aligned}
& J_{L Q G}=\lim _{T \rightarrow \infty} E\left\{J_{L Q R}\right\}, \\
& J_{L Q G}=\lim _{T \rightarrow \infty} E\left\{\int_{0}^{\infty}\left[\boldsymbol{z}^{T}(t) Q \boldsymbol{z}(t)+\boldsymbol{u}^{T}(t) R \boldsymbol{u}(t)\right] d t\right\} .
\end{aligned}
$$

The matrices $Q$ and $R$ are the weights that make the adjustment of the index $J_{L Q G}$ and, as discussed above, it has directly influence in the resulting controller. These matrices have the following characteristics:

$$
Q=Q^{T} \geq 0, \quad R=R^{T}>0
$$

\subsubsection{Solution of the LQG problem}

The solution of the LQG problem is given by the separation principle, which states that the optimal result is achieved by the following procedure: firstly, an optimal estimate $\hat{x}$ of the state $x$ is obtained; Secondly, the estimated state is used as if it were the real and exact measure of the state of the system to solve the quadratic linear regulator problem. Therefore, the whole solution of the method is reduced to two sub-problems that have their known solutions.

\subsubsection{The Kalman filter}

The solution of the first sub-problem, which is the optimal state estimation, is given by the Kalman filter theory. The structure of the Kalman filter is equal to the structure of a state observer, except for the way as the gain matrix $K_{f}$ is obtained. To design a Kalman filter it is necessary to have the plant model, that is the state space matrices, and have access to the control signals, $u$, and output signals, $y$, which are the filter inputs. The output, according to the objective of the sub-problem, is the estimated state vector $\hat{x}$ (MORRIS, 1976), (PAPOULIS; PILLAI, 2002). 
Figure 37: The Kalman filter.

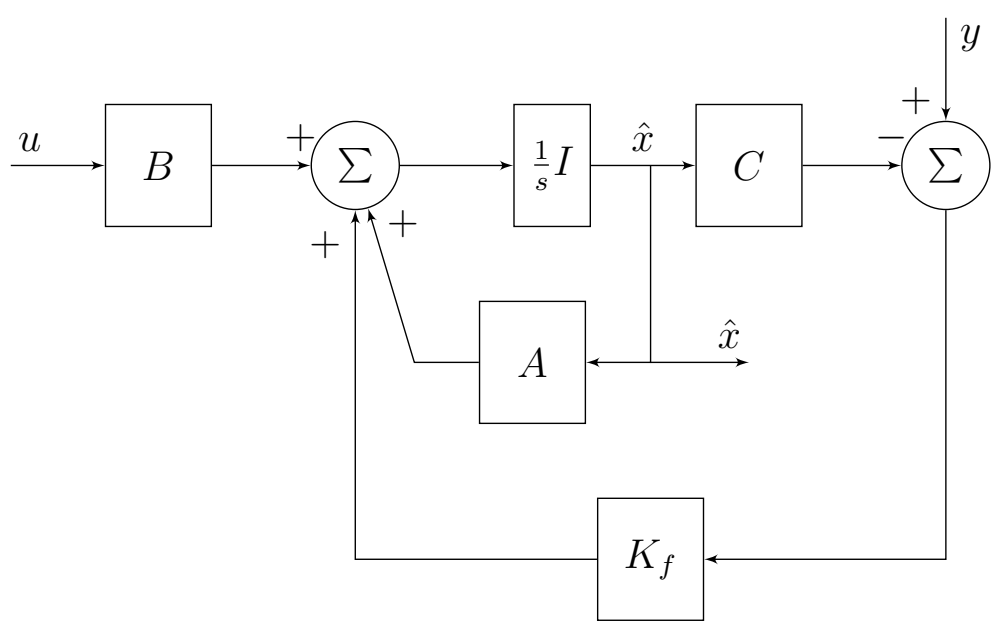

Source: Author.

The gain matrix $K_{f}$ is given by:

$$
K_{f}=P_{f} C^{T} \Theta^{-1}
$$

where $P_{f}$ is the unique positive semidefinite solution of the algebraic Riccati equation:

$$
P_{f} A^{T}+A P_{f}-P_{f} C^{T} \Theta^{-1} C P_{f}+\Gamma W \Gamma^{T}=0
$$

and $P_{f}=P_{f}^{T} \geq 0$.

\subsubsection{The LQR regulator}

The solution of the second sub-problem is to find the control signal that minimizes the quadratic performance index in Equation 6.31. The hypothesis to solve this problem is that the control signal $u$ is a linear function of the states:

$$
u=-K_{c} x
$$

and the optimal state feedback matrix $K_{c}$ is given by:

$$
K_{c}=R^{-1} B^{T} P_{c}
$$

where, just as in the Kalman filter, $P_{c}$ satisfies Ricatti's algebraic equation, which is dual to Equation 6.34:

$$
A^{T} P_{c}+P_{c} A-P_{c} B R^{-1} B^{T} P_{c}+Q=0
$$

and $P_{c}=P_{c}^{T} \geq 0$. 


\subsubsection{LQG analysis}

The Figure 38 shows the internal structure of the controller $K_{L Q G}$ formulated by solving the problem LQG. Its configuration is given by the serial connection of the Kalman filter with the feedback gain of the states.

Figure 38: Structure of the LQG compensator.

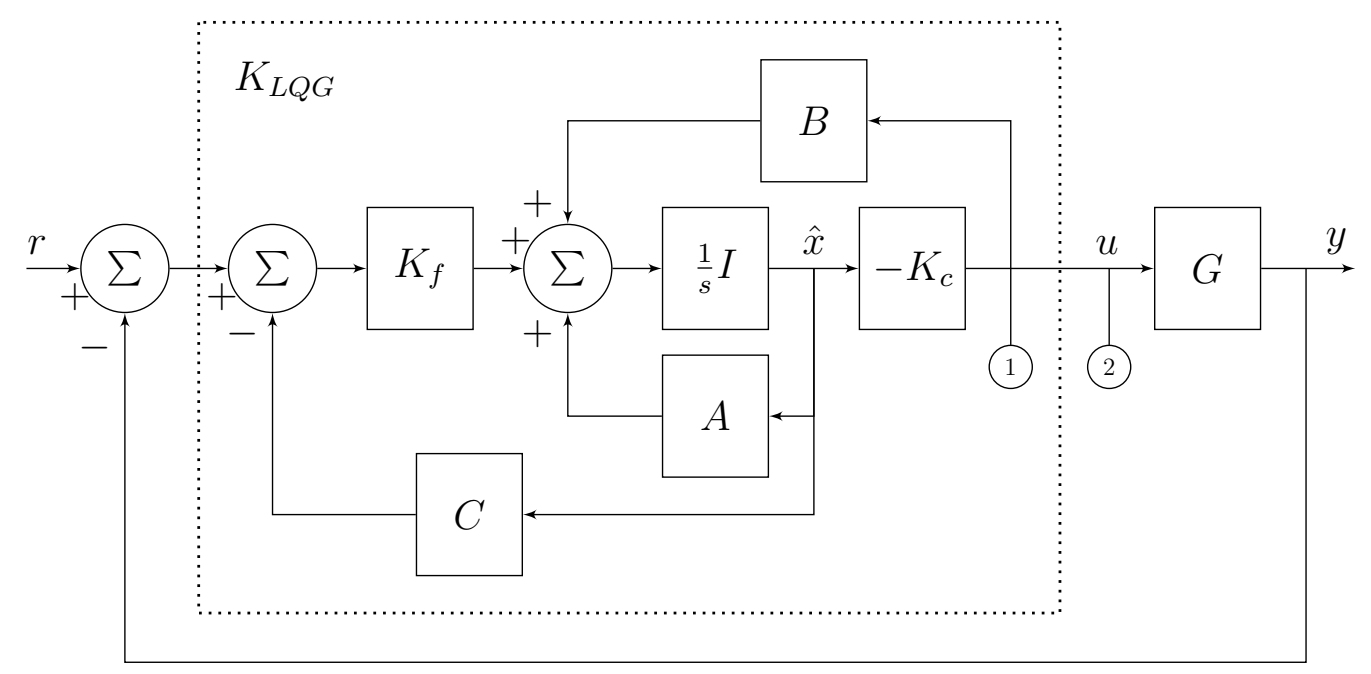

Source: Author.

Once the gain matrices $K_{f}$ and $K_{c}$ are calculated according to the previous sections, one can construct the state-space realization for the controller $K_{L Q G}$ (ZAREI et al., 2007) by the following equation:

$$
\begin{aligned}
& A_{L Q G}=A-B K_{c}-K_{f} C, \\
& B_{L Q G}=K_{f}, \\
& C_{L Q G}=-K_{c}, \\
& D_{L Q G}=0 .
\end{aligned}
$$

\subsubsection{LTR - loop transfer recovery}

Although the Kalman filter and the linear quadratic regulator have good results for robust stability and robust performance, the combination of the two solutions, as shown in Figure 38, does not guarantee robustness. A common practice to address such a problem is to make the dynamics of the Kalman filter much faster than the dynamics of state feedback. However, Doyle and Stein have shown that in some cases, as the state observer dynamics becomes faster, the stability margin is reduced (DOYLE; STEIN, 1979). Then, it was developed a way to design the Kalman filter so that all state feedback properties 
are retrieved at the plant input: it is called the Loop Transfer Recovery (LTR) (CRUZ, 1996).

An informal and simplified explanation for using the LTR is this: the performance and robustness properties at the plant input are determined by the return function at point 2, marked in Figure 38, If it is supposed that all the states of the plant are available for feeding back through $K_{c}$, which is the desired scenario, the Kalman filter has no influence, and the return ratio at point 1 is given by $-K_{c}(s I-A)^{-1} B$. This function becomes simple because the Kalman filter has a dynamic response so fast that it is not excited by the control signal $u$, that is, the filter neglects the control signal. If the only signal acting on the system is $u$ and the matrices $A, B$, and $C$ of the Kalman filter characterize the plant very well, the signal entering the gain matrix $K_{f}$ is zero. However, the points 1 and 2 in Figure 38 have very different values for real systems. Therefore, the LTR method forces the return function at point 1 to approximate the value of point 2, placing the Kalman filter's eigenvalues at the zeros of the plant (DOYLE; STEIN, 1979). For more information about the LTR process see (MACIEJOWSKI, 1989) and (GU; PETKOV; KONSTANTINOV, 2005).

Taking the following considerations into account:

- the pair $(A, B)$ is controllable;

- the pair $(A, C)$ is observable;

- there is no zeros in the right complex half-plane;

The gain matrix of the LQR problem is:

$$
K_{c}=\frac{1}{\rho} B^{T} P_{c}
$$

where $P_{c}$ is the solution of the Equation 6.37.

In this way, as $\rho$ approaches zero (cheap control), (CRUZ, 1996) proves that the open-loop system $G(j \omega) K(j \omega)$, considering the controller $K(j \omega)$, approaches to:

$$
G_{K F}=C(s I-A)^{-1} K_{f},
$$

where $K_{f}$ is the gain matrix of the Kalman filter and this process is known as the loop transfer recovery (LTR). The resulting closed-loop system is shown in Figure 39. 
Figure 39: System approach to $\rho \rightarrow 0$.

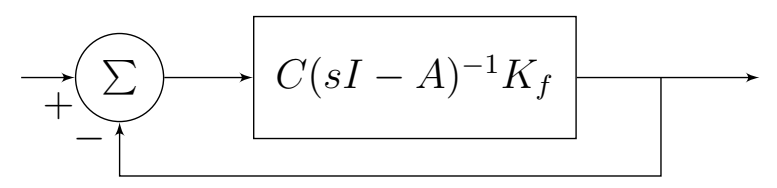

Source: Author.

Then, the solution consists of choosing the parameters of design $W, \Theta$ and $\Gamma$, so that the gain of $G_{K F}$ satisfies the conditions of robustness of stability and the conditions of robustness of the performance as presented in the previous section. In this work, it will be considered that $W=1$ and $\Theta=\mu$. Under these conditions, (CRUZ, 1996) shows that if $\mu$ is small enough, then the following approximate formula is valid:

$$
\left|G_{K F}(j \omega)\right| \approx \frac{1}{\sqrt{\mu}}\left|C(j \omega I-A)^{-1} \Gamma\right|
$$

Therefore, the Equation 6.41 is important to the process of the loop shaping of openloop response, because it relates directly the design parameters, $\mu$ and $\Gamma$ and the response of $G(j \omega) K(j \omega)$ at the frequencies where the performance barriers are situated (CRUZ, 1996).

\section{6 $\quad H_{\infty}$ loop-shaping method}

There are several formulations on how to use cost functions in robust controller design. The $\mathrm{S} / \mathrm{KS}$ and $\mathrm{S} / \mathrm{T} / \mathrm{KS}$ design methods are well known, which are optimization routines so that the controller $\mathrm{K}$ are designed satisfying robust stabilization and performance requirements. They are useful design methods, but the representations of perturbations are limited by the condition on the number of right-half complex poles (GU; PETKOV; KONSTANTINOV, 2005). The $H_{\infty}$ loop-shaping method is an alternative design procedure, in which the uncertainty is described by the perturbations directly on the coprime factors of the nominal model, as in the Figure 32 and Figure ??. Some of the advantages of this method is that it could relax the restrictions on the number of right-half plane poles, produce no pole-zero cancellations between the nominal model and the controller and it does not require an iterative process to obtain an optimal solution, decreasing the computational cost (MACFARLANE, 1990). This section presents the main results in this theory and the design procedure to obtain the respective controller. 


\subsubsection{Robust stabilization}

Let's build a left coprime factorization of the plant model $G$ as:

$$
G=M^{-1} N
$$

where $M$ is square and $\operatorname{det}(M) \neq 0$. A left coprime factorization is said to be normalized if and only if:

$$
M M^{H}+N N^{H}=I, \quad \forall s,
$$

where $M^{H}$ is the Hermitian conjugate of $M$, i.e. $M^{H}(s)=M^{T}(-s)$. Consider a minimal realization of $G$ :

$$
G(s)=C(s I-A)^{-1} B+D=:\left[\begin{array}{l|l}
A & B \\
\hline C & D
\end{array}\right] .
$$

The normalized left coprime factorization can be built from the minimal realization of $G$ as a state-space construction with the solution of the generalized filter algebraic Riccati equation:

$$
\left(A-B D^{T} R^{-1} C\right) Z+Z\left(A-B D^{T} R^{-1} C\right)^{T}-Z C^{T} R^{-1} C Z+B\left(I-D^{T} R^{-1} D\right) B^{T}=0,
$$

where $R=I+D D^{T}$ and $Z \geq 0$ is the unique stabilizing solution. If $H=-\left(Z C^{T}+\right.$ $\left.B D^{T}\right) R^{-1}$, then from (GU; PETKOV; KONSTANTINOV, 2005):

$$
\left[\begin{array}{ll}
N & M
\end{array}\right]=\left[\begin{array}{c|c}
A+H C & B+H D H \\
\hline R^{-\frac{1}{2}} C & R^{-\frac{1}{2}} D R^{-\frac{1}{2}}
\end{array}\right],
$$

is the normalized left coprime factorization of $G$ such that $G=M^{-1} N$.

A perturbed plant transfer function can be written as depicted in Figure 32, where the transfer function is given by:

$$
G_{\Delta}=\left(M+\Delta_{M}\right)^{-1}\left(N+\Delta_{N}\right)
$$

The Figure 40 shows the connection between the perturbed plant and the controller. The family of possible plants is defined in Equation 6.48 and the objective of the design is to find a robust compensator $K$, in order to stabilize, not only the nominal model $G$ 
but all possible plants contained into that family:

$$
\Omega_{\epsilon}=\left\{\left(M+\Delta_{M}\right)^{-1}\left(N+\Delta_{N}\right):\left\|\left[\Delta_{M}, \Delta_{N}\right]\right\|_{\infty}<\epsilon\right\}
$$

Figure 40: Coprime factor robust stabilization problem.

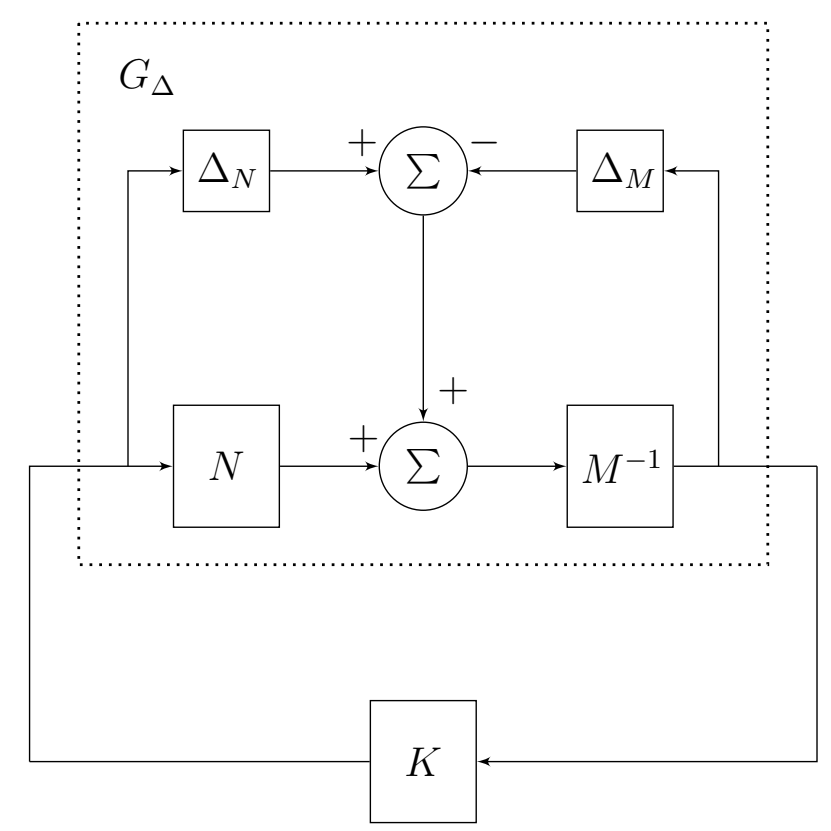

Source: Author.

The stability margin is given by $\epsilon$ in Equation 6.48. After some calculations and using the small-gain theorem, the feedback system shown in Figure 40 is robustly stable if and only if:

$$
\left\|\left[\begin{array}{c}
K(I-G K)^{-1} M^{-1} \\
(I-G K)^{-1} M^{-1}
\end{array}\right]\right\|_{\infty} \leq \epsilon^{-1} .
$$

Therefore, it is desired to maximize the robust stability of the closed-loop system. In order to reach this objective, it is necessary to minimize the following cost function:

$$
\gamma=:\left\|\left[\begin{array}{c}
K \\
I
\end{array}\right](I-G K)^{-1} M^{-1}\right\|_{\infty} .
$$

The lowest achievable value of $\gamma$ and the corresponding maximum stability margin $\epsilon$ are given by (MCFARLANE; GLOVER, 1992) as:

$$
\gamma_{\min }=\epsilon_{\max }^{-1}=\left(1-\left\|\left[\begin{array}{ll}
N & M
\end{array}\right]\right\|_{H}^{2}\right)^{-\frac{1}{2}}
$$


where $\|.\|_{H}$ denotes the Hankel norm. From (MCFARLANE; GLOVER, 1992), the Equation 6.51 can be rewritten as:

$$
\gamma_{\min }=\epsilon_{\max }^{-1}=\left(1+\lambda_{\max }(Z X)\right)^{\frac{1}{2}}
$$

where $\lambda_{\max }$ denotes the maximum eigenvalue of $Z X$. For a minimal state-space realization $(A, B, C, D)$ of $G, Z$ is the unique positive definite solution to the algebraic Riccati equation:

$$
\left(A-B S^{-1} D^{T} C\right) Z+Z\left(A-B S^{-1} D^{T} C\right)^{T}-Z C^{T} R^{-1} C Z+B S^{-1} B^{T}=0
$$

where $R=I+D D^{T}$ and $S=I+D^{T} D$ and $X$ is the unique positive definite solution of the following algebraic Riccati equation:

$$
\left(A-B S^{-1} D^{T} C\right)^{T} X+X\left(A-B S^{-1} D^{T} C\right)-X B S^{-1} B^{T} X+C^{T} R^{-1} C=0 .
$$

Notice that when $D=0$ (i.e., an strictly proper system), there is a considerable simplification on the above calculation. Hence, the controller which guarantees:

$$
\left\|\left[\begin{array}{c}
K \\
I
\end{array}\right](I-G K)^{-1} M^{-1}\right\|_{\infty} \leq \gamma,
$$

for a specified $\gamma>\gamma_{\text {min }}$, is given by:

$$
K_{\infty}=\left[\begin{array}{c|c}
A+B F+\gamma^{2}\left(L^{T}\right)^{-1} Z C^{T} C & \gamma^{2}\left(L^{T}\right)^{-1} Z C^{T} \\
\hline B^{T} X & 0
\end{array}\right]
$$

where:

$$
\begin{gathered}
F=-S^{-1} B^{T} X, \\
L=\left(1-\gamma^{2}\right) I+X Z .
\end{gathered}
$$

\subsubsection{The loop-shaping design procedure}

In order to guarantee performance, an adjust of the open-loop response is conducted before calculating the robustifying controller (BOUKHNIFER; FERREIRA, 2007). The sequence of design is as follows:

- it is added to the plant $G(s)$ to be controlled a pre-compensator $W_{1}$ and/or a postcompensator $W_{2}$ (see Figure 41a). Then, the singular values of the nominal plant for 
MIMO systems or the gain for SISO systems is shaped to give a desired open-loop shape. The combination aims to improve the performances of the system such as $G_{s}=W_{2} G W_{1}$, as in Figure 41b,

- Through coprime factorization of $G_{s}$, it is applied the previous results to calculate $\epsilon_{\max }$, and then synthesize a stabilizing controller $K_{\infty}$, which ensures a value slightly lower than the $\epsilon_{\max }$.

- The final feedback controller is obtained by combining the $H_{\infty}$ controller with the shaping functions $W_{1}$ and $W_{2}$ such that $K=W_{1} K_{\infty} W_{2}$, as in Figure 41c.

Figure 41: The loop-shaping design procedure.

(a)

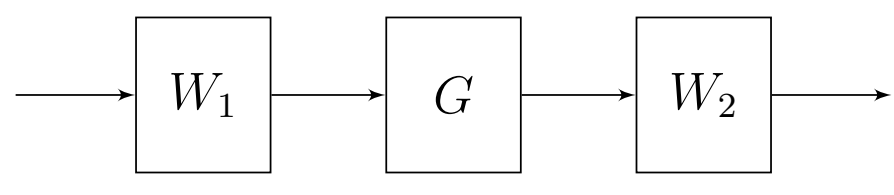

(b)

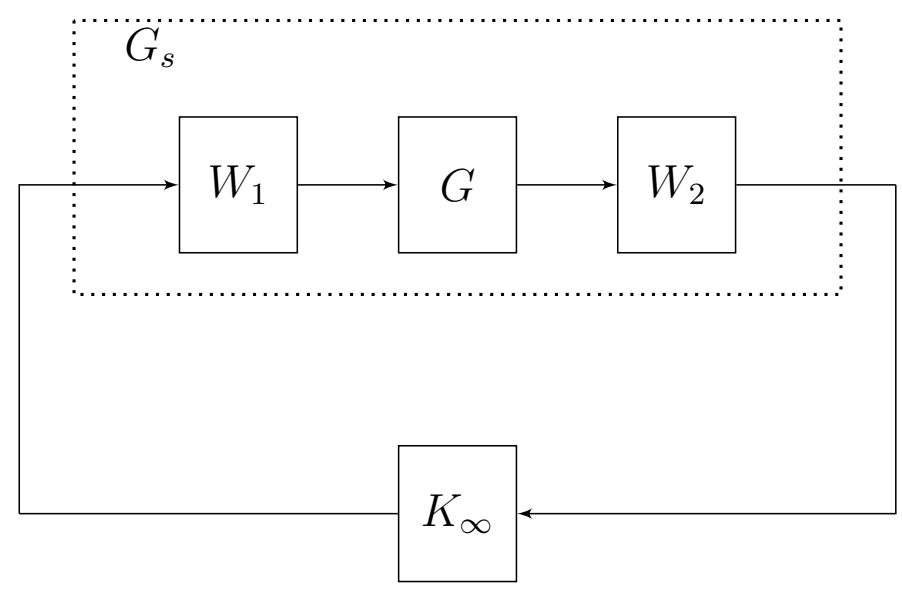

(c)

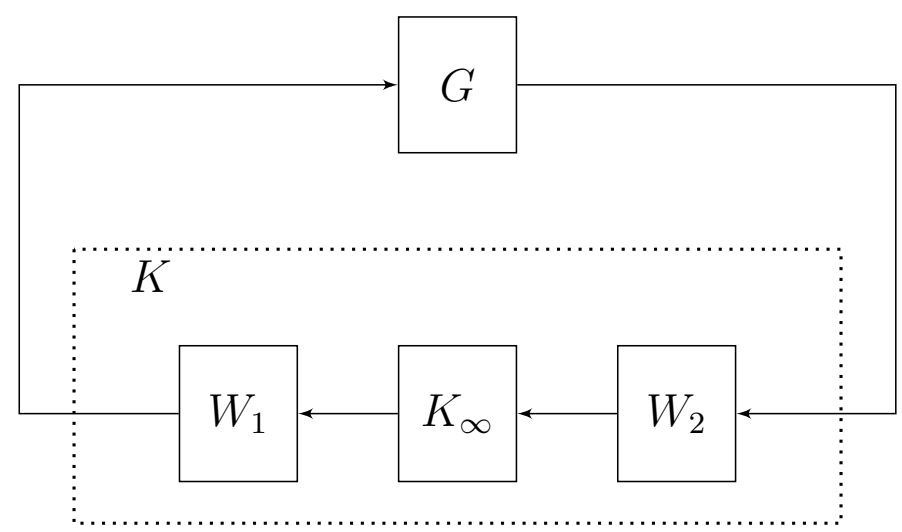

Source: Author. 
The next chapter will present the results obtained for both control methods described in this chapter and applied to the active anti-roll system discussed in Chapter 5. The robustness and performance of the controllers are analyzed and the response of the vehicle without anti-roll system and with passive anti-roll bar are also reported. 


\section{SIMULATION RESULTS}

This chapter shows the results obtained for the three models built in Chapter 4: a vehicle without anti-roll system, a vehicle with passive anti-roll bar and a vehicle with an active anti-roll bar. Firstly, the design objectives are listed in Section 7.1. Section 7.2 presents the order reduction of the active system in order to make it suitable to the proposed control designs: LQG/LTR method and $H_{\infty}$ loop-shaping method. As consequence, a modeling error is built considering the difference among the full-order finite element model and the reduced order model. The three models have in common the lateral acceleration, $a_{y}$, and the road profiles, $z_{r r}$ and $z_{r l}$, as the disturbances reflected at the plant. As mentioned in Section 5.2, the lateral acceleration is given by the Equation 5.60. The road profiles are neglected in this work due to the lateral acceleration to represent the main disturbance related to the roll dynamics in cornering. In Section 7.3. the results of the LQG/LTR design is presented. Likewise, the $H_{\infty}$ loop-shaping is designed and the results are presented in Section 7.4. Finally, Section 7.5 shows the comparison of the controllers according to three maneuvers: step steer test, sinusoidal steer test and single lane change. Afterthat, the performance is analyzed through the RMS value of the roll angle.

\subsection{Design objectives}

1. Robust Stability. It is verified by the check of the condition written in Equation 6.24 .

2. Disturbance Rejection. It is considered that a lateral acceleration does not happen in frequencies above $0.7[\mathrm{~Hz}](\approx 4.4[\mathrm{rad} / \mathrm{s}])$.

3. Measurement error. The robust design considers a sensor which ensures that the measurements are reliable up to $50[\mathrm{~Hz}](\approx 314.2[\mathrm{rad} / \mathrm{s}])$.

4. Input Magnitudes. In real active anti-roll systems, there is a torque module which is 
designed to provide the sufficient torque to act on vehicle. Therefore, it is assumed that the torque necessary to control the system are totally available. In addition, the values of the control signal (i.e. the torque) will be checked if it presents values compatible with the practice.

\subsection{Model reduction and uncertainty model}

Typically, models provided by FEM presents large number of d.o.f.'s and the design of the controller becomes a complicated task. In this case study, the order reduction through truncation was applied by the Hankel singular values of the balanced representation only for the active system, which is the unique to have the controller for the anti-roll bar. It has 196 state variables, 1 control inputs and 3 disturbance inputs. The reduction results, in balanced coordinates, are presented in Figure 42, which illustrates the Hankel singular values considering all inputs influencing the output, the vehicle's roll angle.

Figure 42: Hankel singular values vs. their corresponding state.

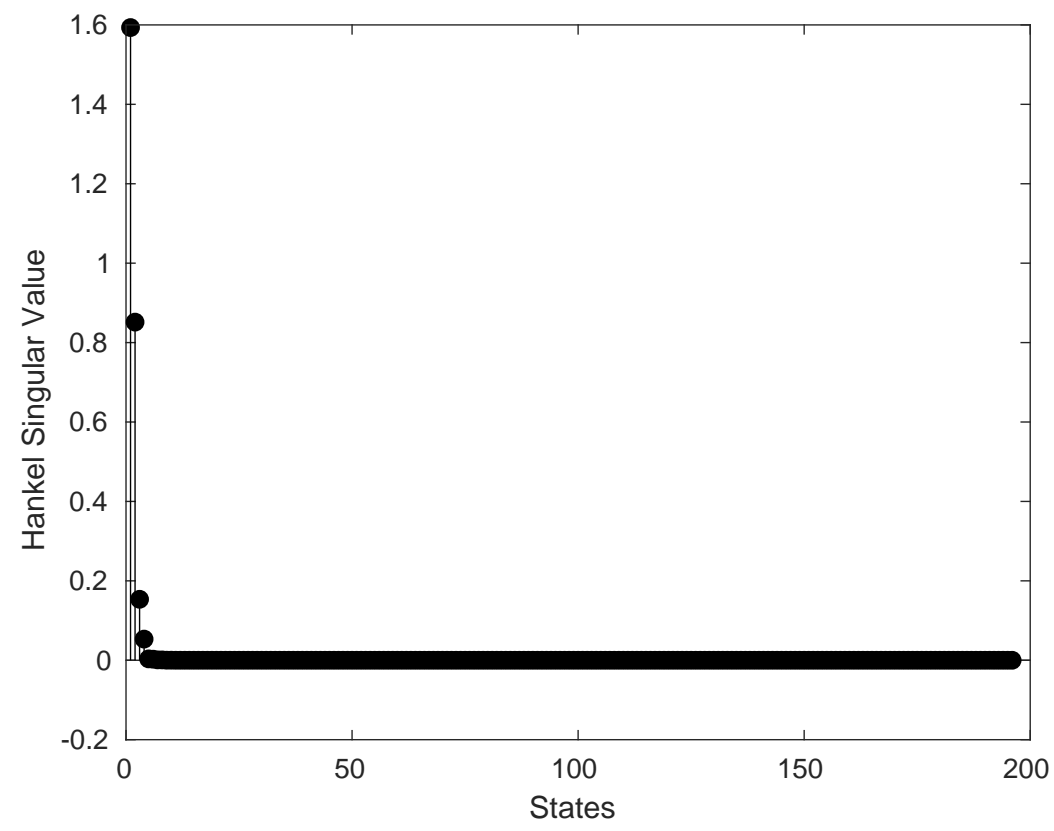

Source: Author.

They show that the first two states has the most influence on the output of the system. The first state has the Hankel Singular Value of 1.593 and the second of 0.8516 . The third state has the Hankel singular value of 0.1536 , which is about six times smaller than the second state value. Therefore, the truncation point chosen is the second state. The Figure 43 shows the first thirty Hankel singular values and the states in the gray 
region are discarded in the reduced model. The Figure 44 shows the Bode diagram from the torque input to the roll angle for the original model with 196 states and the reduced model with the two more relevant states to design the controller. They show that the reduction of model kept the low frequency characteristic of the original system below 500 radians per second, which is very similar to a second-order system and justify the choice for two states in reduction order procedure. The resonance peaks seen in the original model represents the natural frequencies inserted by the flexible nature of the anti-roll bar and they lie at higher frequencies. These resonance peaks were ignored by the chosen truncation point in the reduction action. All the requirements of the Section 7.1, in terms of barriers, are below $500 \mathrm{rad} / \mathrm{s}$, and to guarantee that the obtained controller work in the complete model, the robust stability condition must be satisfied since poorly damped and high frequency poles will be neglected, assumed as unmodeled dynamics.

Figure 43: Hankel singular values vs. order of the reduced model (first 30 states).

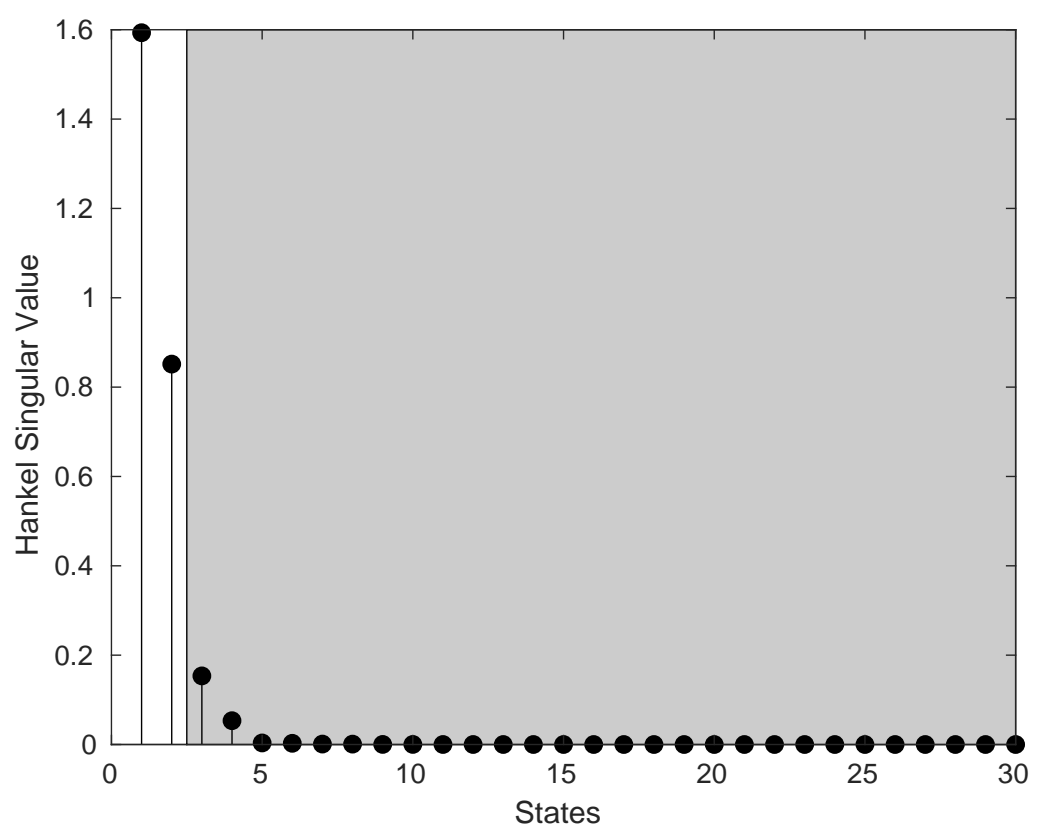

Source: Author.

\subsubsection{Uncertainty model}

The construction of the upper limit to $\left|\varepsilon_{M}(j \omega)\right|$, called $e_{M}(\omega)$, is established as the relative difference between the full-order FEM model treated here as the real system $G_{\Delta}(s)$, which includes 196 states, and the reduce-order model, consisting of 2 states and treated as the nominal system $G(s)$. Then, the function $e_{M}(\omega)$ is reached by the multiplicative uncertainty: 
Figure 44: Magnitude plot of the original and reduced models for active anti-roll bar.

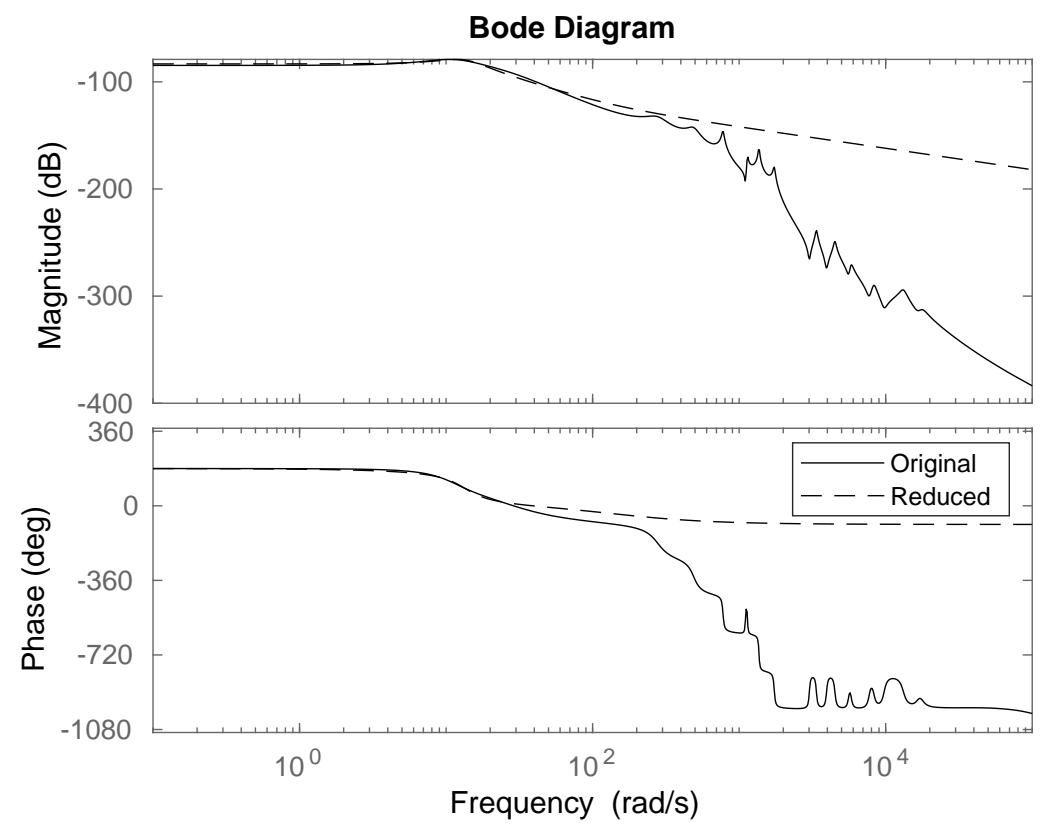

Source: Author.

$$
e_{M}(\omega)=\left|\frac{G_{\Delta}(j \omega)-G(j \omega)}{G(j \omega)}\right|
$$

where the $|$.$| represents the magnitude operator. The resulted e_{M}(\omega)$ is shown in Figure 45

Figure 45: Error function $e_{M}(\omega)$ by multiplicative definition of uncertainty.

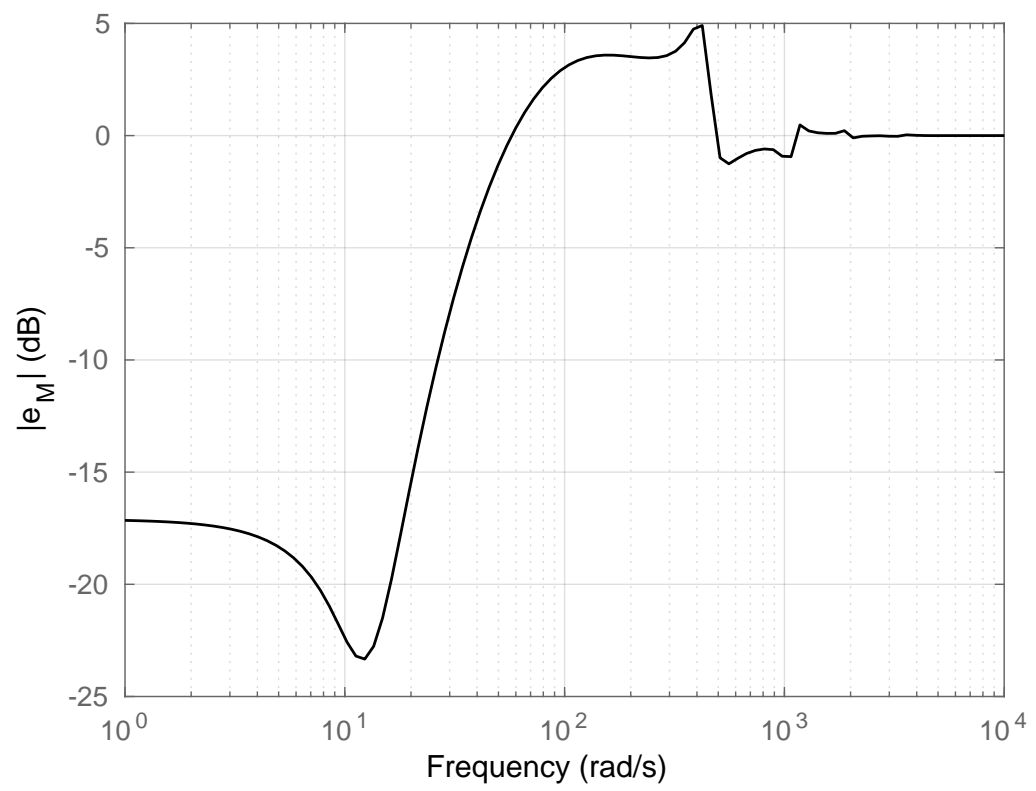

Source: Author. 
Therefore, the designs are made based on the nominal system $G(s)$ and the robustness analysis of the control system compares the open-loop response $G(s) K(s)$ with the barriers discussed earlier together with the error function $e_{M}(\omega)$ to state about robust performance and robust stability.

\subsection{LQG/LTR controller}

The controller is obtained based on the state-space realization $(A, B, C, D)$ of the nominal system. In the first step, the Kalman filter problem is dealt by solving the Kalman filter ARE as in Equation 6.34. In this case, the gain matrix $K_{f}$ is calculated as in Equation 6.33 by choosing the weighting matrices $\Theta$ and $\Gamma$, which are given by:

$$
\Theta=\rho I, \quad \Gamma=B
$$

where $\rho=0.3 \times 10^{-10}$. In the second step, the LQR problem is solved by estimating the optimal state feedback matrix $K_{c}$, as shown in Equation 6.36. Hence, the Equation 6.37 is figured out by setting the weighting matrices $R$ and $Q$ as:

$$
R=\mu I, \quad \Gamma=C^{T} C
$$

where $\mu$ is defined by the LTR process.

The Figure 46 shows three iterations considering the values of $\mu$ as $1,10^{-8}$ and $10^{-16}$.

The Figure 47 shows the open-loop response $G(s) K(s)$ and barriers defined by design objectives. The LQG/LTR controller leads the system to satisfy the performance requirements for disturbance and measure error.

The controller's transfer function obtained is given in Equation 7.4. In order to improve steady-state response of the controller, an integrator was added throughout the design. A fourth order controller was obtained by the LQG/LTR method:

$$
K(s)=-\frac{1.217 \times 10^{9} s^{2}+1.863 \times 10^{10} s+2.413 \times 10^{11}}{s^{4}+241.5 s^{3}+2.93 \times 10^{4} s^{2}+1.518 \times 10^{6} s} .
$$

The Figure 48 shows the poles and zeros of the nominal closed-loop control system. The system is nominally stable where all poles has negative real parts and it possess a pair of conjugate zeros which is near of a pair of conjugate poles, practically canceling each other's actions. The Figure 49 shows the gain of both sensitivity $S(s)$ and closed-loop transfer function $T(s)$. This shows that the bandwidth, the $-3 \mathrm{~dB}$ frequency of $T(s)$, has 
Figure 46: Loop Transfer Recovery (LTR).

(a) $\mu=1$

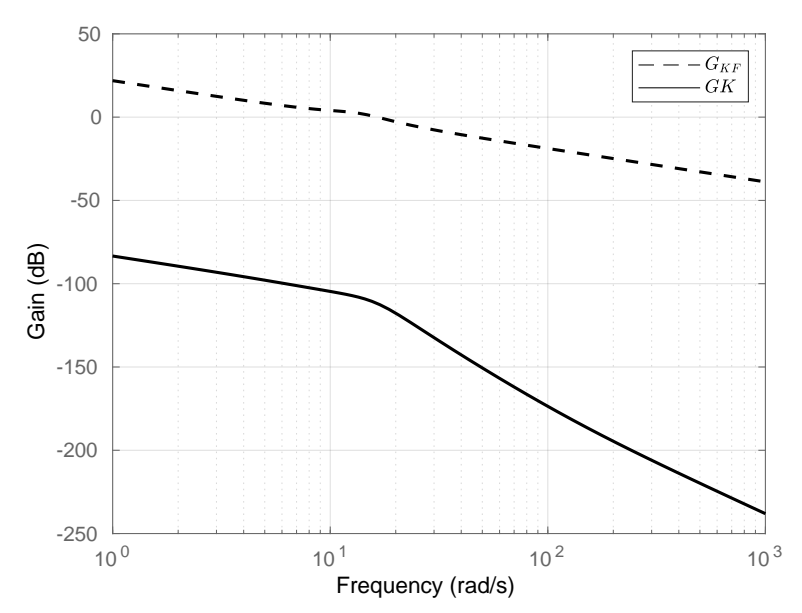

(b) $\mu=10^{-8}$

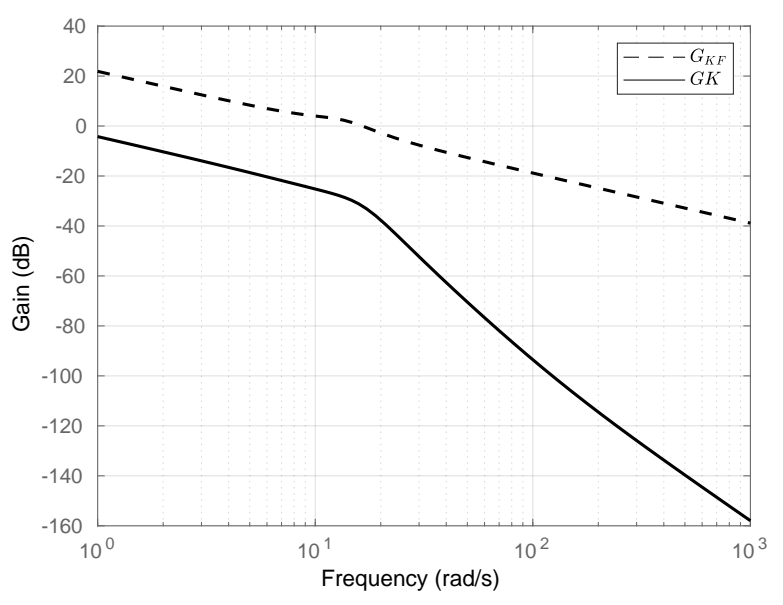

(c) $\mu=10^{-16}$

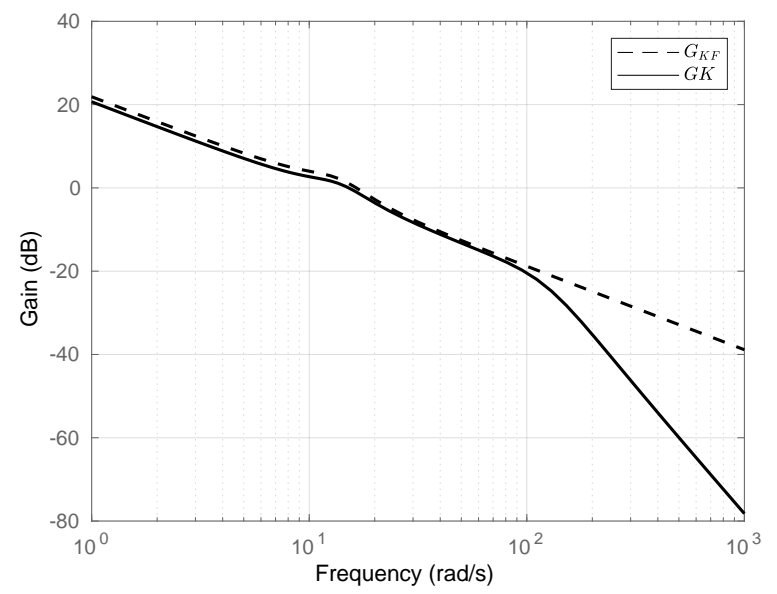

Source: Author. 
Figure 47: Open-loop response and the performance barriers.

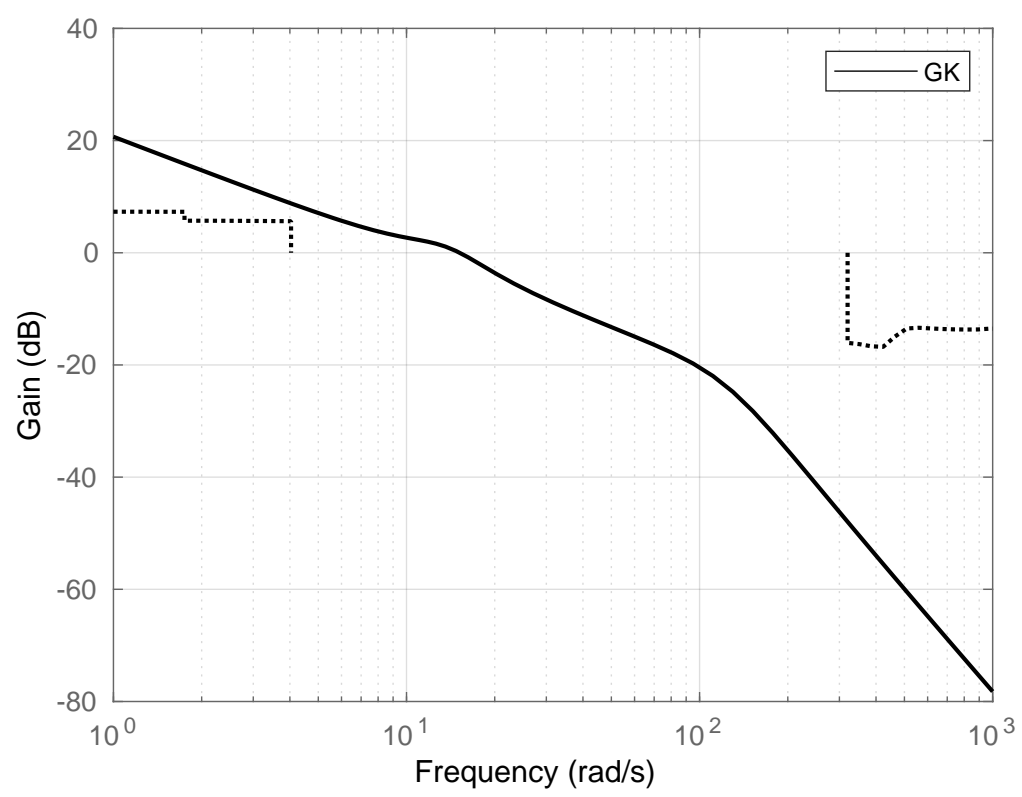

Source: Author.

been approximately about $3.1 \mathrm{rad} / \mathrm{s}$, and that of $S(s)$ being between about $2.2 \mathrm{rad} / \mathrm{s}$.

Figure 49: Sensitivity and complementary sensitivity function for LQG/LTR.

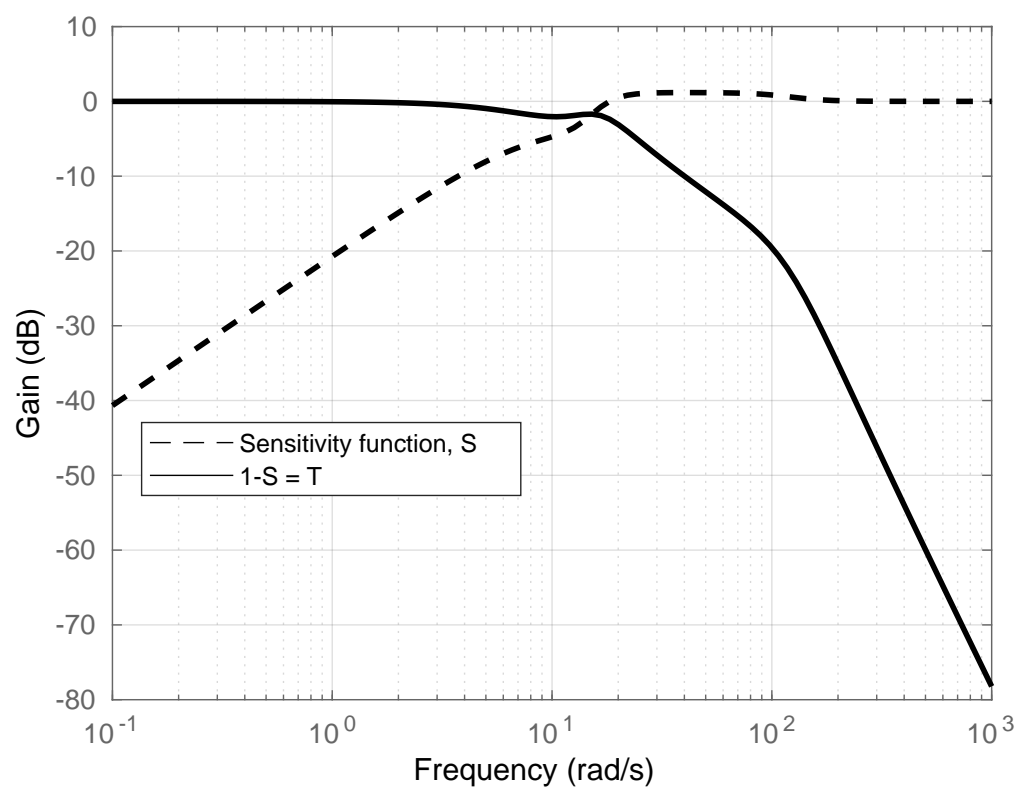

Source: Author.

The robust stability condition is verified at the Figure 50. 
Figure 48: Poles (x) and zeros (o) of the closed-loop system with nominal plant for LQG/LTR.

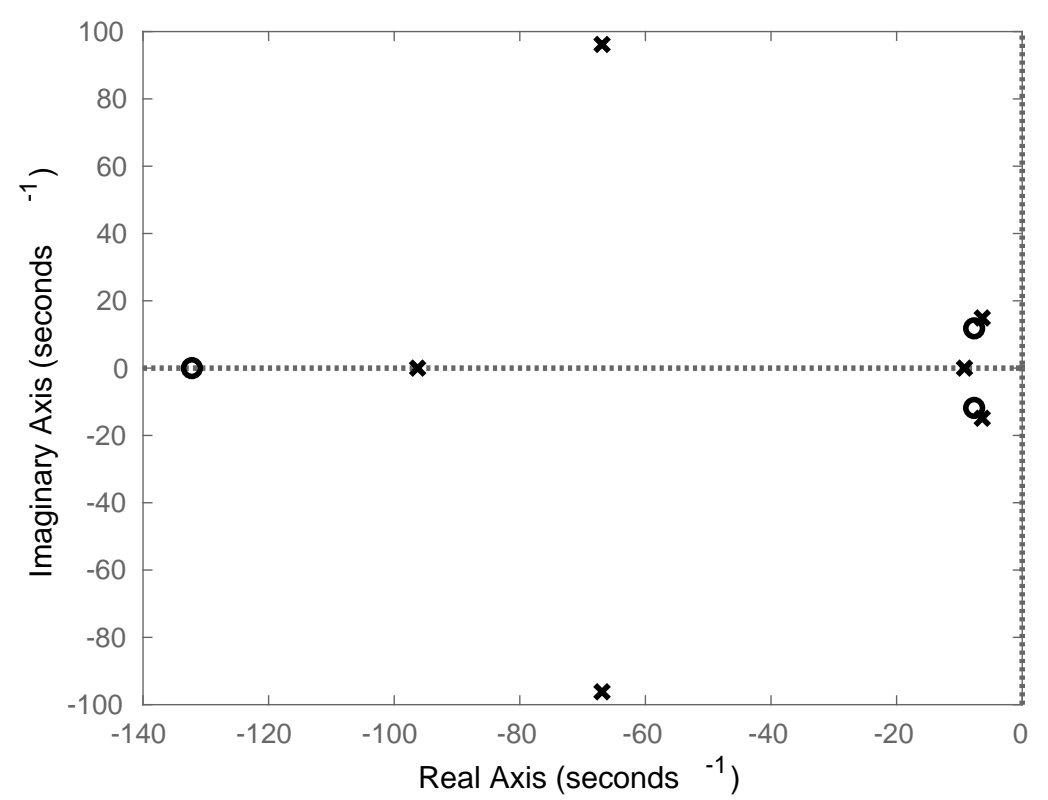

Source: Author.

Figure 50: Robust stabilization condition for LQG/LTR.

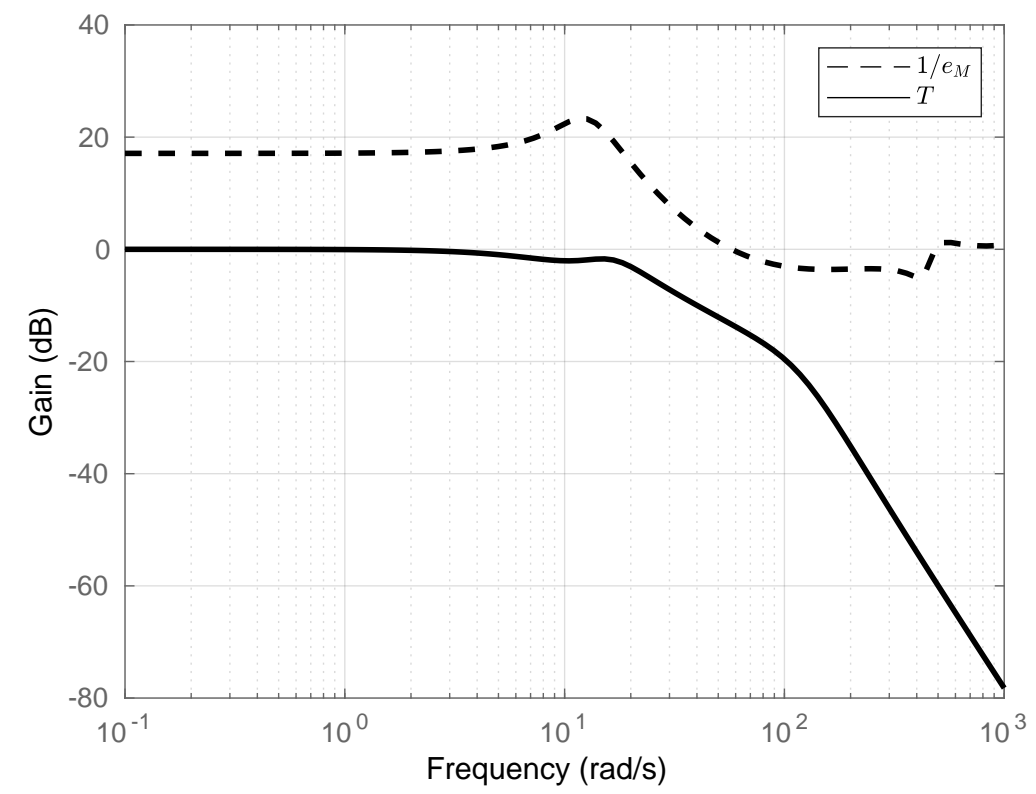

Source: Author. 


\section{$7.4 \quad H_{\infty}$ loop-shaping controller}

It is followed the design procedure given in Section 6.6.2. The first step is select the pre- and post-compensators to obtain the desired shape plant, which justify the name loop-shaping in the method. The gain values of the nominal plant $G(s)$ and the shaped plant $G_{s}(s)=W_{2}(s) G(s) W_{1}(s)$ are shown in Figure 51. The nominal case indicates a need for extra low frequency gain to give good disturbance rejection. Thus, in order to build the shaped plant $G_{s}$, it is used only the weighting function $W_{1}$, so that;

$$
W_{1}(s)=\frac{20}{s}, \quad W_{2}(s)=1
$$

The crossover is adjusted to $20 \mathrm{rad} / \mathrm{s}$ in order to put the open-loop transfer function inside the limits proposed by performance barriers.

Figure 51: Nominal plant, shaped plant and barriers for HLS.

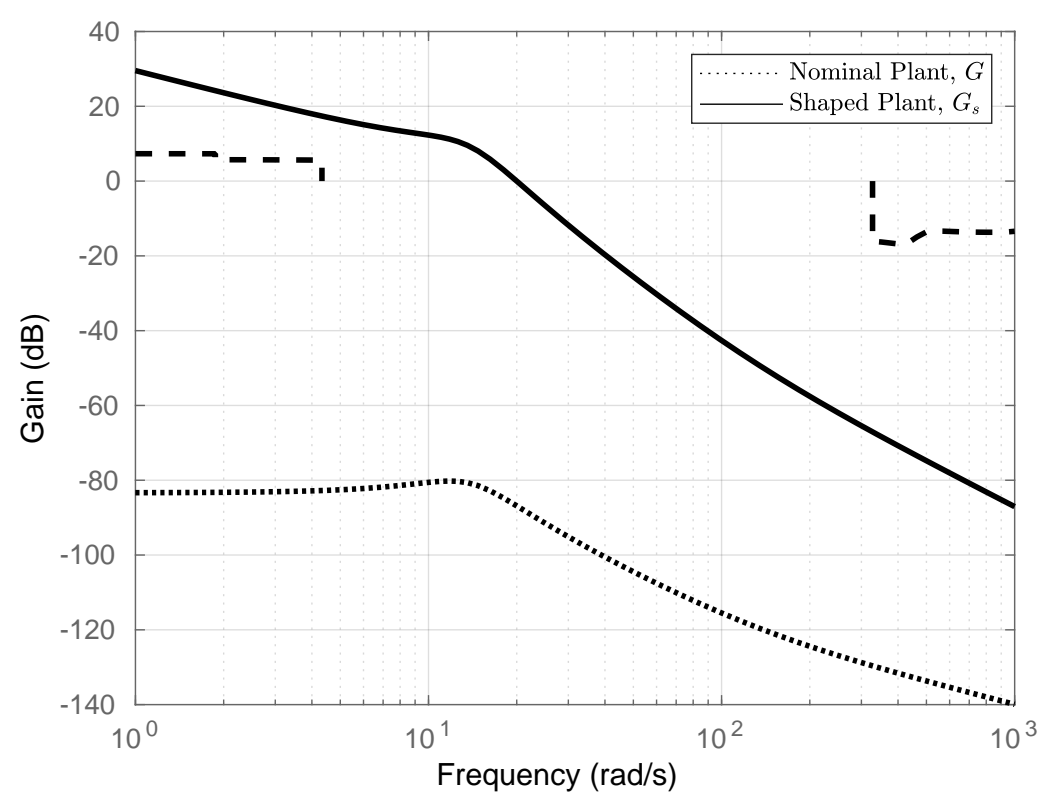

Source: Author.

After the shaping of the plant, through coprime factorization of $G_{s}$, it is calculated the $\gamma_{\text {min }}$, which is 2.7302 in this case. According to Skogestad, this values indicates that the shaped plant is compatible with robust stability, where $\gamma_{\min } \leq 4$ (SKOGESTAD; POSTLETHWAITE, 2007). Figure 52 shows the open-loop response with the margins given in function of $\gamma_{\min }$.

The controller's transfer function obtained by the $H_{\infty}$ loop-shaping design is given in 
Figure 52: Open-loop response for HLS.

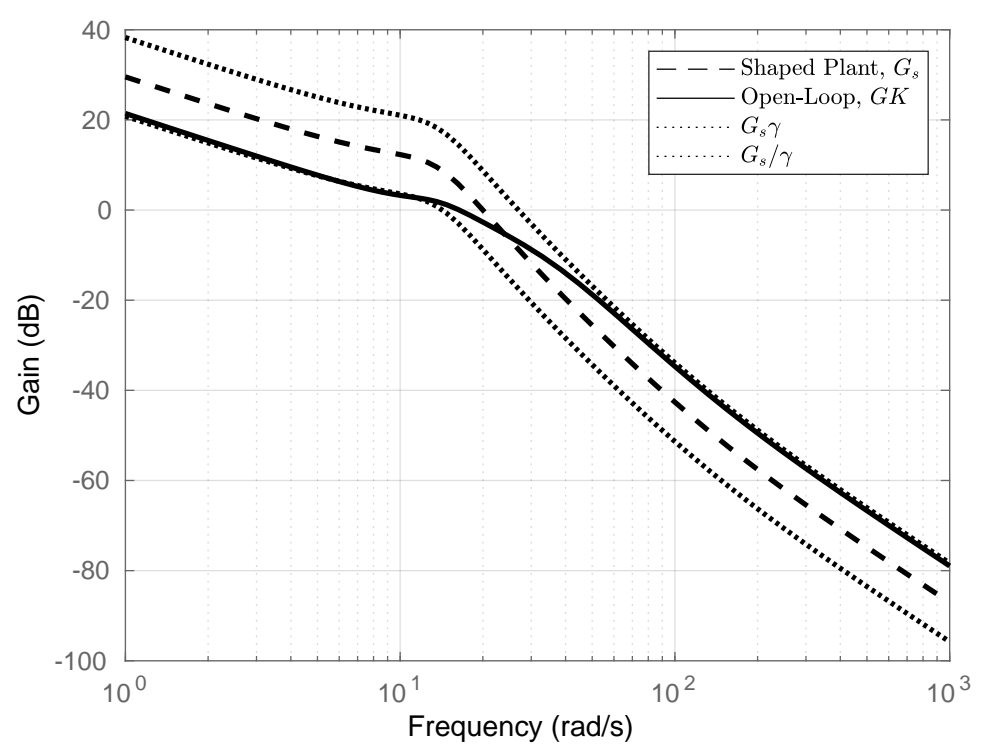

Source: Author.

Equation 7.6. A third order controller was obtained by this method:

$$
K(s)=-\frac{1.118 \times 10^{6} s^{2}+1.577 \times 10^{7} s+2.001 \times 10^{8}}{s^{3}+49.08 s^{2}+1155 s} .
$$

The nominal stability can be verified by and poles and zeros of the closed-loop transfer function $T(s)$. The Figure 53 shows that all poles and zeros has negative real parts and, therefore, the closed-loop nominal system is stable.

Figure 53: Poles $(\times)$ and zeros $(\mathrm{o})$ of the closed-loop system with nominal plant for HLS.

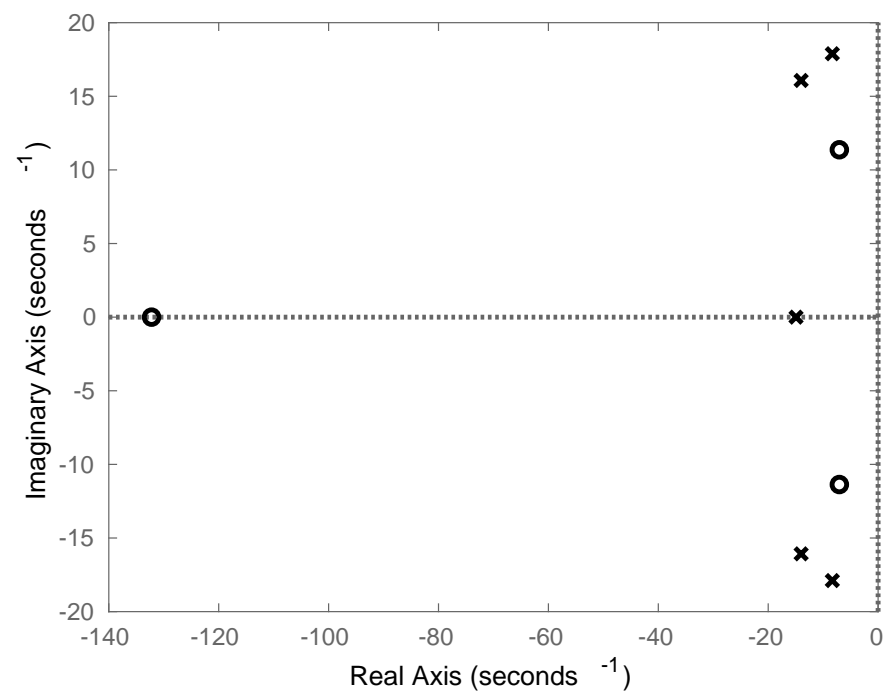

Source: Author. 
The Figure 54 shows the gain of the sensitivity $S(s)$ and the gain of the closedloop transfer function $T(s)$. The bandwidth for the $H_{\infty}$ Loop-shaping design has been approximately about $27 \mathrm{rad} / \mathrm{s}$, whereas the LQG/LTR procedure reached a bandwidth about $3.1 \mathrm{rad} / \mathrm{s}$. The sensitivity function $S(s)$ has a rejection range of signals until 2.0 $\mathrm{rad} / \mathrm{s}$.

Figure 54: Sensitivity and complementary sensitivity function for HLS.

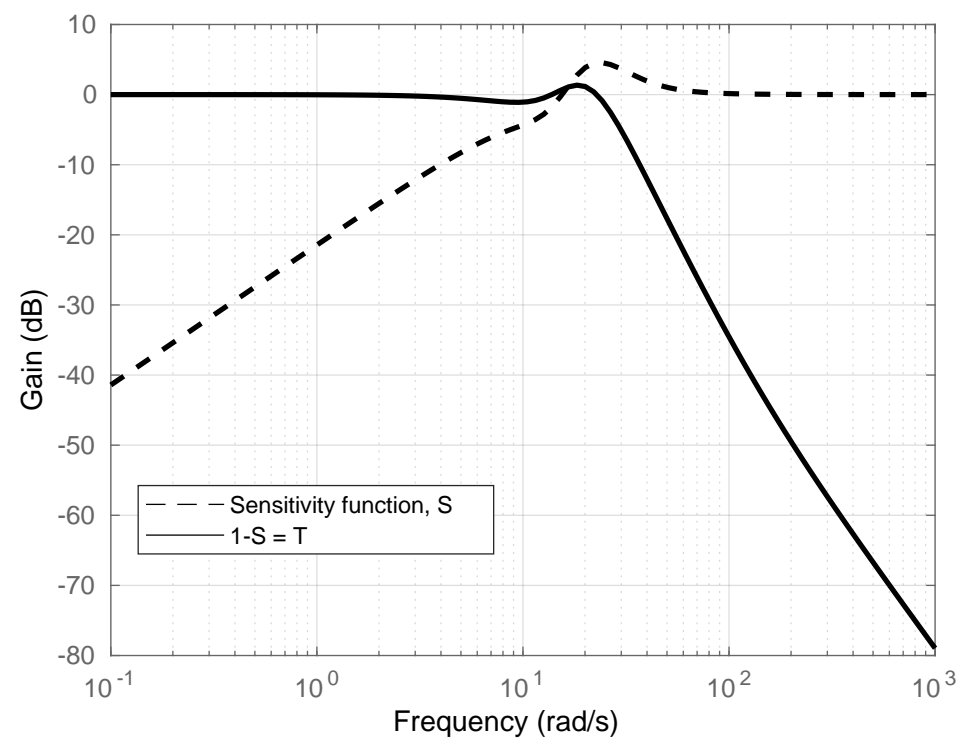

Source: Author.

Figure 55: Robust stability condition for HLS.

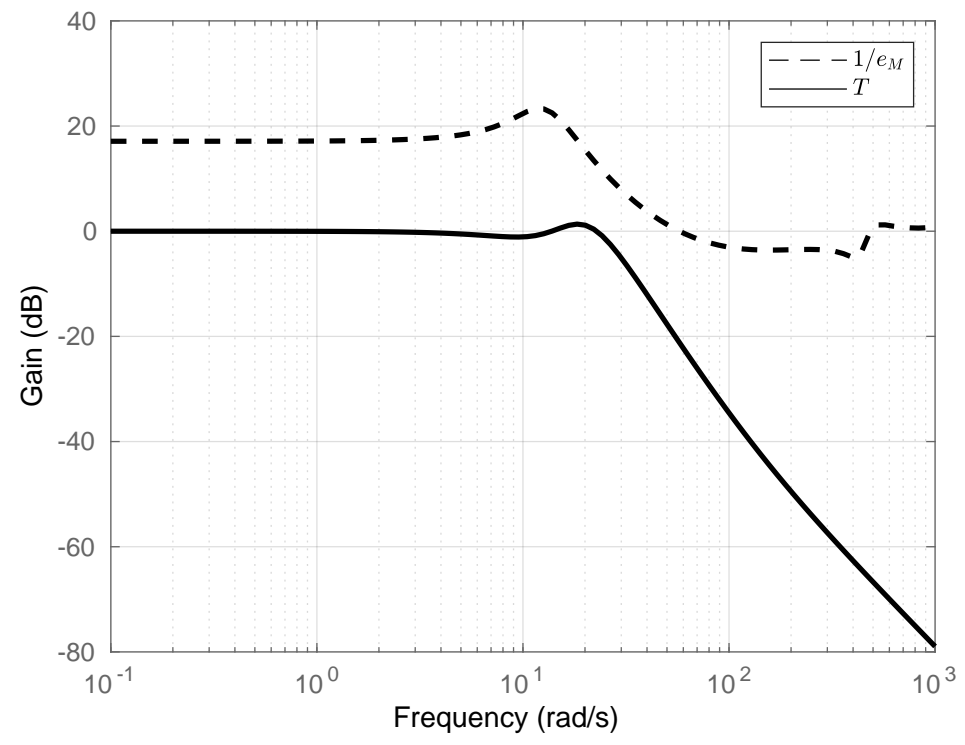

Source: Author. 
The closed-loop transfer function presents $\|T\|_{\infty}=1.1684$, which is the resonance peak at $18.4 \mathrm{rad} / \mathrm{s}$, and $\|S\|_{\infty}=1.6928$ at $23.7 \mathrm{rad} / \mathrm{s}$. The robust stability condition is verified at the Figure 50 where the closed-loop transfer function is below the inverse of $e_{M}(\omega)$ for all frequencies in range satisfying the relation in Equation 6.20 .

\subsection{Comparison of the controllers}

Three maneuvers are devised to study the behavior of the systems: step steer test, sinusoidal steer test and the single lane change. The results are obtained by using simulation software Matlab $\AA$ and Simulink $囚$. Simulations are carried out for three configurations of the same vehicle: without anti-roll bar, with passive anti-roll bar and with active anti-roll bar. The Figure 56 shows the block diagram used to simulate the uncontrolled systems and the Figure 57 for the controlled system. All the models are simulated with its respective non-linear state equations.

Figure 56: Block diagram for the uncontrolled system.

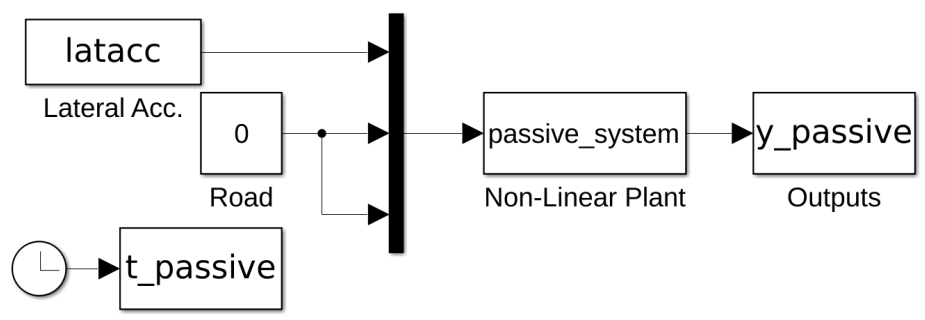

Source: Author.

Figure 57: Block diagram for the controlled system.

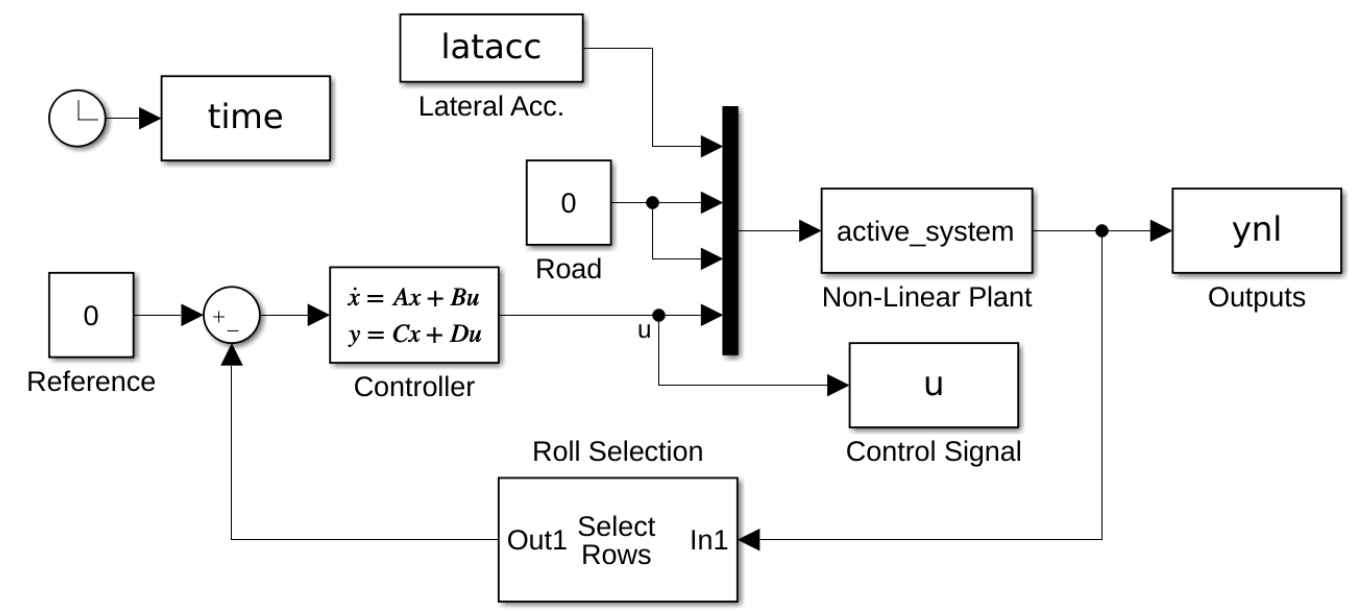

Source: Author. 


\subsubsection{Step steer test}

In order to evaluate steady state performance, an step steer test has been carried out with vehicle at speed of $60 \mathrm{~km} / \mathrm{h}$ by using the steering input at the handwheel, changing it from 0 degrees to 60 degrees after 2 seconds, as shown in Figure 58a, This step steer input generates the lateral acceleration shown in Figure 58b, which it will be a disturbance input to the hybridized models, and not a directly step as a disturbance.

Figure 58: Step command in handwheel and lateral acceleration in $60 \mathrm{~km} / \mathrm{h}$ step steer test.

(a)

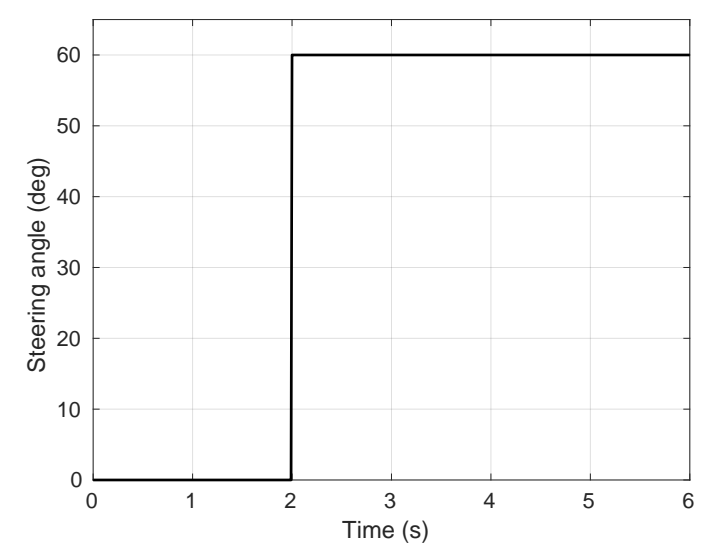

(b)

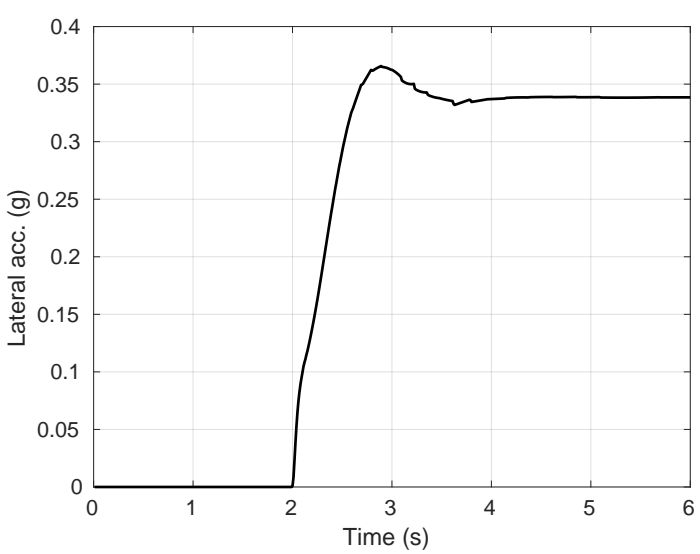

Source: Author.

The main behavior of anti-roll bars under these types of application are the bending of the levers and the torsion of the central beam (see Figure 6). At first, let's analyze the passive anti-roll bar results when subjected to the lateral acceleration. The Figure 60 shows the displacement in the y-direction of the nodes 1,3 and 5, as illustrated in the Figure 59. The nodes, as seen before, are the points of geometric discretization used in FEM, where the displacements and rotations will be solved.

Figure 59: Nodes d.o.f. of the passive anti-roll bar.

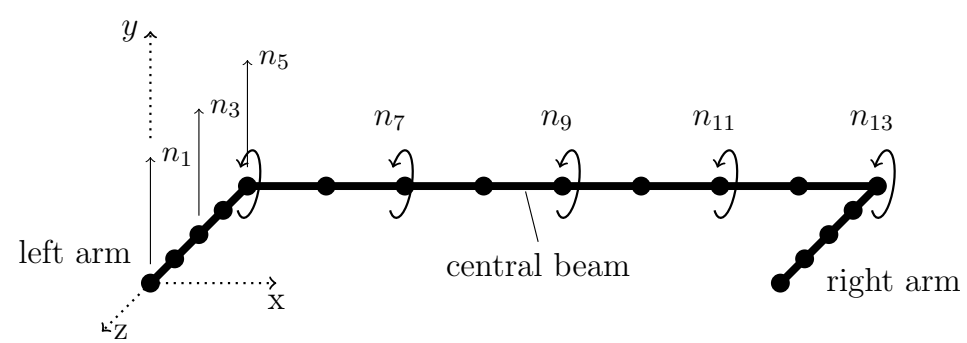

Source: Author. 
It is seen that the node 1 has, after the transient, a displacement of approximately $0.52 \mathrm{~mm}$ in the negative direction and the node 5 has a displacement of $0.18 \mathrm{~mm}$ negative. This difference shows that the natural bending of the passive anti-roll bar is occurring under lateral acceleration generated by the step steer command. In Figure 11, the lateral acceleration is positive for the left direction. As the input is positive, the sprung mass of the vehicle are dislocated to the left, forcing the right suspension to lower. This behavior justify the negative values of the y-direction displacement. These results are symmetric for the other side, but in the opposite direction.

Figure 60: Passive anti-roll bar: bending in y-direction in $60 \mathrm{~km} / \mathrm{h}$ step steer test.

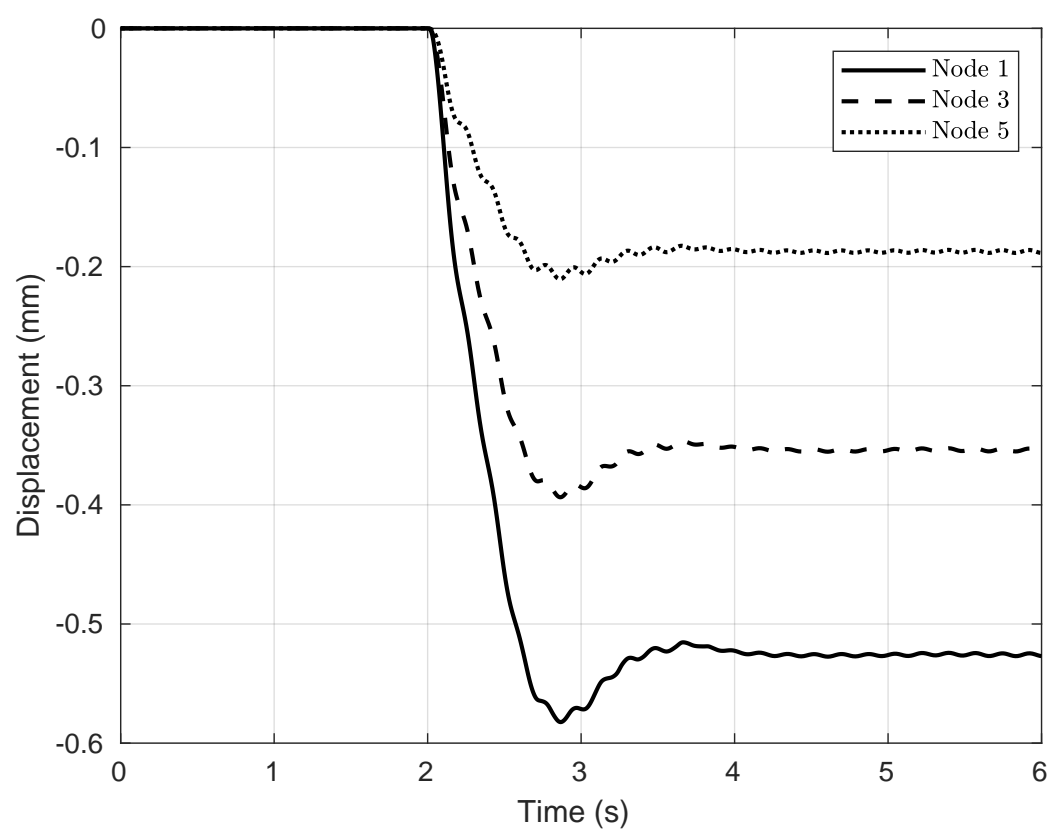

Source: Author.

The Figure 61 shows the results of torsion for the nodes 5, 7, 9, 11 and 13. As it can be seen, the results are symmetric because when the lateral force is applied in the positive direction, as in Figure 11, the vehicle's sprung mass is dislocated to the left forcing the right suspension to lower, and at the same time, forcing the left suspension to lift. In this way, the middle point of the bar, where the node 9 is located, experienced zero torsion. At the tip of the central beam (nodes 5 and 13 in Figure 59), the torsion $\theta$ of the passive bar is, approximately, 0.18 degrees in magnitude after the transient. 
Figure 61: Passive anti-roll bar: torsion in $60 \mathrm{~km} / \mathrm{h}$ step steer test.

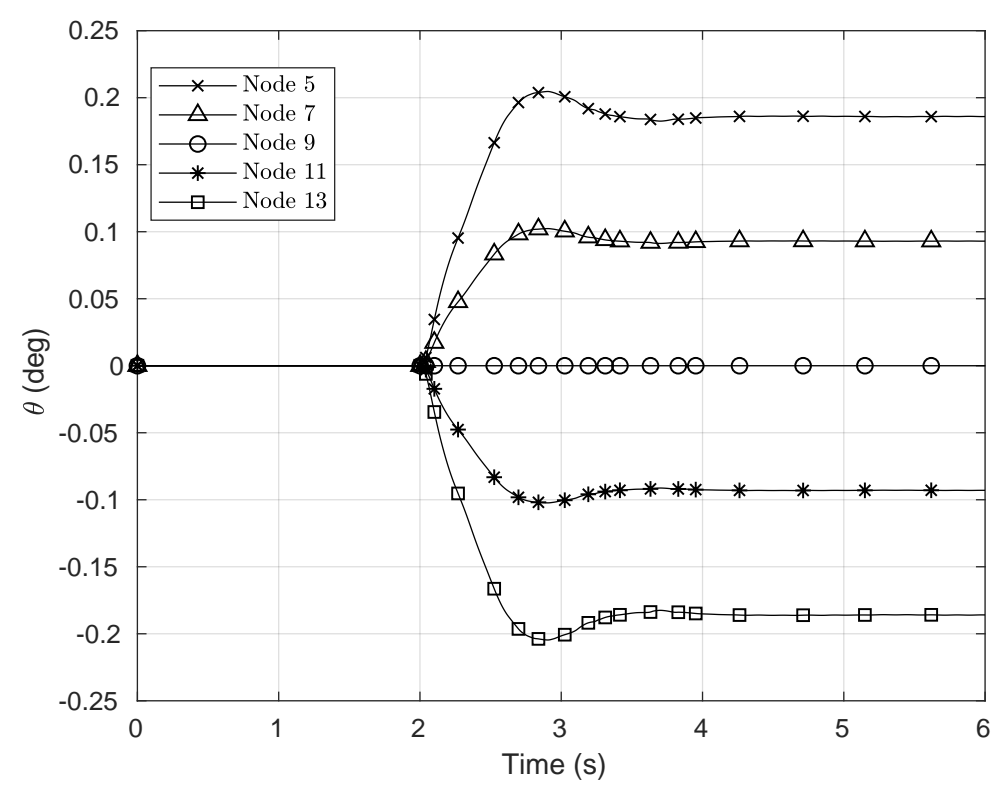

Source: Author.

For the active case, where a controlled electric motor has been inserted, the results are quite different. In Figure 62, it is shown the nodes where the results are taken for. The Figure 63 shows the bending behavior through the nodes 1, 3 and 5 for the two control methods applied: LQG/LTR and $H_{\infty}$ methods. Notice that the bending is in opposite direction when compared with the passive case in Figure 60. The electric motor placed at the middle of the bar justifies the change of direction. When the lateral acceleration forces the left suspension to lower, which cause a roll angle, the control system provide a torque whereby the central beam twists to compensate the roll angle. In this way, the direction of the displacements change to up. With the control system, the node 1 displace approximately $12.2 \mathrm{~mm}$ after the transient.

Figure 62: Node's d.o.f.'s of the active anti-roll bar.

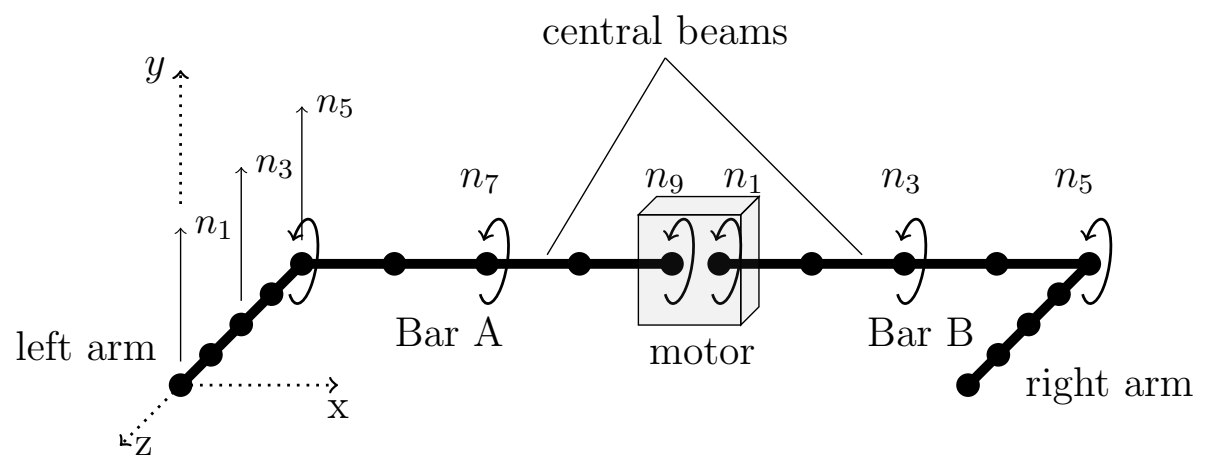

Source: Author. 
Figure 63: Active anti-roll bar: bending in y-direction for the LQG/LTR and HLS in 60 $\mathrm{km} / \mathrm{h}$ step steer test.

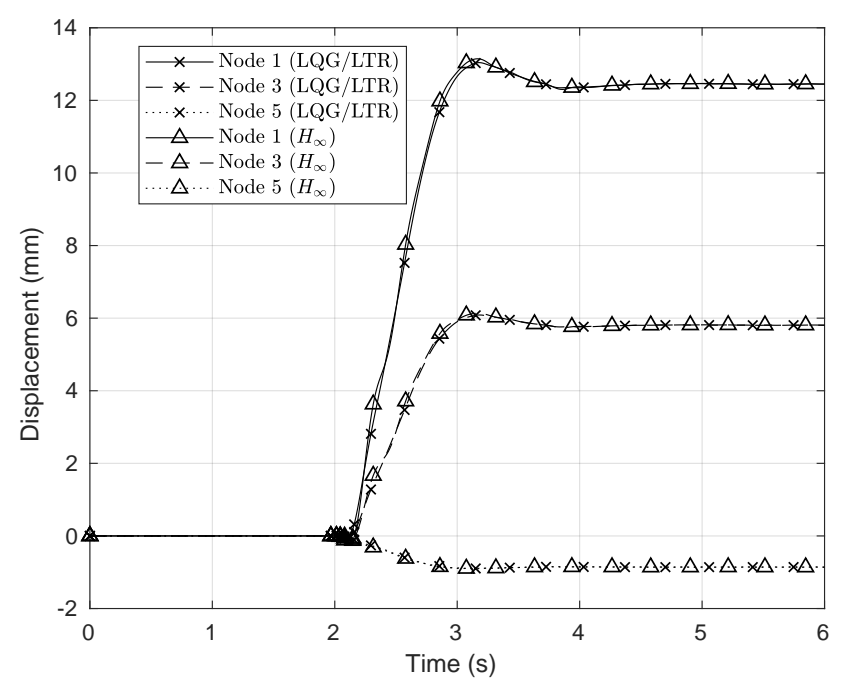

Source: Author.

The torsion results obtained for the active anti-roll bar are presented in the Figure 64 for both synthesized controllers. The torsion experienced by the active bars are bigger than the torsion in the passive one and also are in the opposite direction. As said, the controller (electric motor) twists the bar forcing the suspensions to compensate the roll angle. For the active case, the torsion $\theta$ comes to approximately 8.2 degrees at the tip of the central beams connected to the electric motor (node 9 of the bar A and node 1 of the bar B, as in Figure 62.

Figure 64: Active anti-roll bar: torsion for the LQG/LTR and HLS in $60 \mathrm{~km} / \mathrm{h}$ step steer test.

(a) LQG/LTR controller

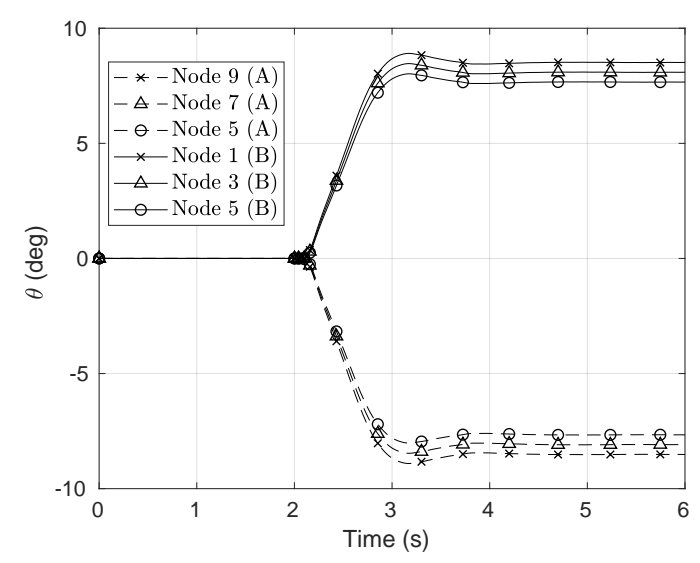

(b) $H_{\infty}$ controller

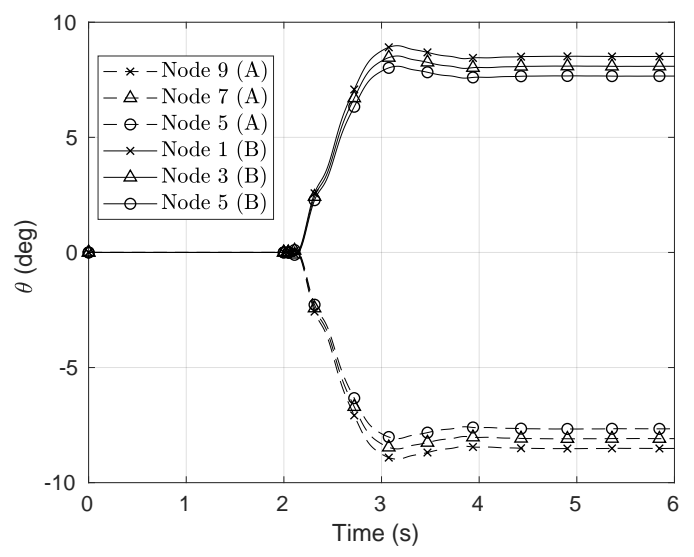

Source: Author. 
As seen in Figure 63 and Figure 64, there is no significant difference between the two controllers applied. Both LQG/LTR controller and $H_{\infty}$ controller shown results very similar for the bars subjected to the step steer test.

The Figure 65 shows the main result of the analysis. The system without anti-roll bar suffers a roll angle of 1.69 degree after the transient under influence of step steer lateral acceleration. When the passive bar is added, the roll angle reduces to 1.32, that represents a decrease of approximately $22 \%$. The third system, with active bar, reduces the roll angle to zero. During the transient, the roll angle reaches about 0.28 degrees for both controllers. Therefore, the time to stabilize the response for two controllers takes about 1.0 second, in Figure 65 between 2.0 and 3.0 seconds.

Figure 65: Roll angle of the model without bar, with passive bar and active bar in 60 $\mathrm{km} / \mathrm{h}$ step steer test.

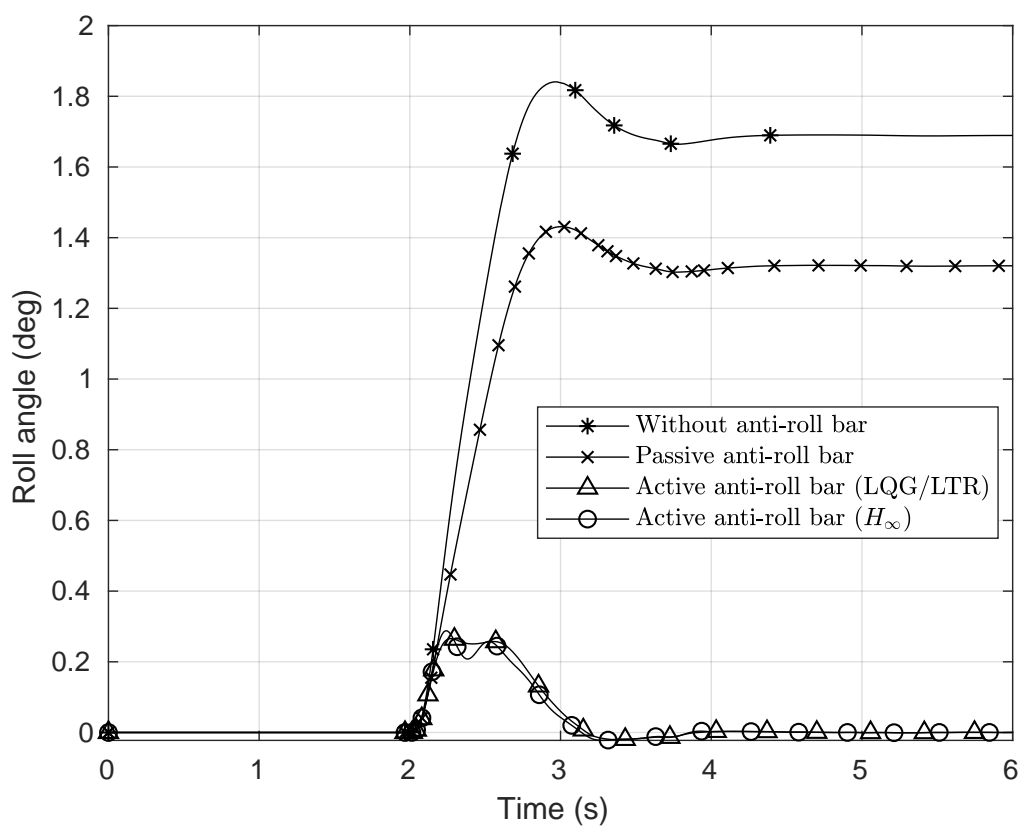

Source: Author.

The roll angle rate is shown in Figure 66. The $H_{\infty}$ controller has an oscillatory behavior moderately higher than the LQG/LTR, as it can be seen between the time 2.0 and 3.0 seconds. This oscillatory behavior could have been predicted by the closed-loop Bode diagram of the $H_{\infty}$ loop-shaping, which has a small resonant peak (Figure 54). However, the amplitude is much smaller than the systems without control. 
Figure 66: Roll rate of the model without bar, with passive bar and active bar in $60 \mathrm{~km} / \mathrm{h}$ step steer test.

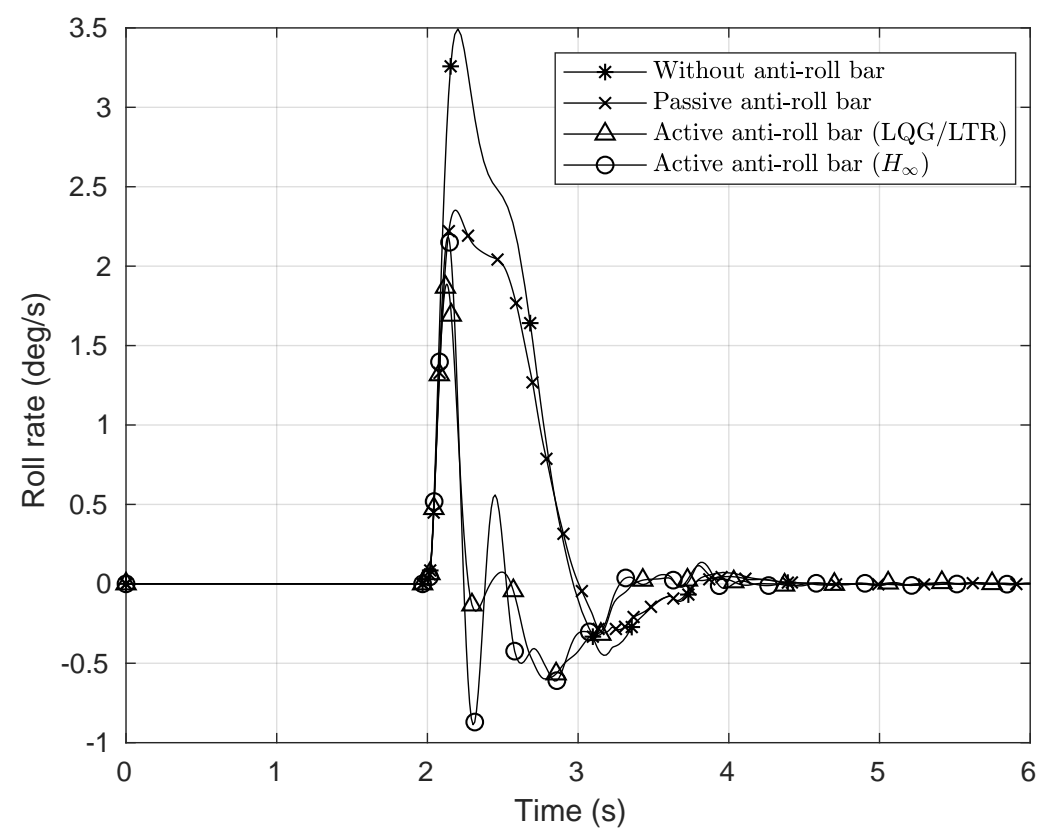

Source: Author.

In order to obtain a quantitative measure of the controllers' performance, it is used the RMS (root mean square). (MUNIANDY et al., 2017). Then, the criterion is then given by:

$$
X_{R M S}=\sqrt{\frac{1}{T} \int_{0}^{T}|x(t)|^{2} d t},
$$

where $x(t)$ is the variable to be analyzed and $T$ is the period of time to be considered. In the following is the table presenting the improvement made by the proposed controllers in comparison with the passive system and the system without anti-roll bar. Table 7 shows the RMS values of the presented variables for $60 \mathrm{~km} / \mathrm{h}$ step steer test.

Table 7: Percentage of improvement in $60 \mathrm{~km} / \mathrm{h}$ step steer test.

\begin{tabular}{|c|c|c|c|}
\hline \multicolumn{2}{|c|}{ Description } & Roll Angle (Degree) & Roll Rate (Degree/s) \\
\hline Without bar & RMS & 0.0229 & 0.0197 \\
\hline Passive bar & RMS & 0.0201 & 0.0152 \\
\hline \multirow{2}{*}{ LQG/LTR } & RMS & 0.0018 & 0.0119 \\
\cline { 2 - 4 } & Improvement (Without/passive) (\%) & $-92.1 /-91.0$ & $-39.6 /-21.7$ \\
\hline \multirow{2}{*}{$H_{\infty}$} & RMS & 0.0017 & 0.0108 \\
\cline { 2 - 4 } & Improvement (Without/passive) (\%) & $-92.6 /-91.5$ & $-45.2 /-28.9$ \\
\hline
\end{tabular}


The LQG/LTR and $H_{\infty}$ controllers improve both their roll angle and roll rate response in comparison with the system without anti-roll bar and also with the passive system. Looking at the roll angle, the LQG/LTR design reduce the RMS values in approximately 91.0\% when compared with the passive system whereas the RMS value of roll rate is decreased in $21.7 \%$. The $H_{\infty}$ controller has an efficiency very similar to the LQG/LTR controller, but demonstrates a slight reduction in RMS values.

The active system presented a dc gain of $299 \mathrm{Nm} /$ degree. It means that the motor provides $299 \mathrm{Nm}$ to compensate one degree of roll angle. This value is comparable with the other ones available in the literature on real active anti-roll system (BHARANE et al. 2014). The Figure 67 shows the control torque applied by motor whereby the roll angle was reduced. While the vehicle was cornering a circular trajectory, the motor kept a torque of $500 \mathrm{Nm}$ to compensate the roll angle.

Figure 67: Control signal for both controllers: torque provided by the electric motor in $60 \mathrm{~km} / \mathrm{h}$ step steer test.

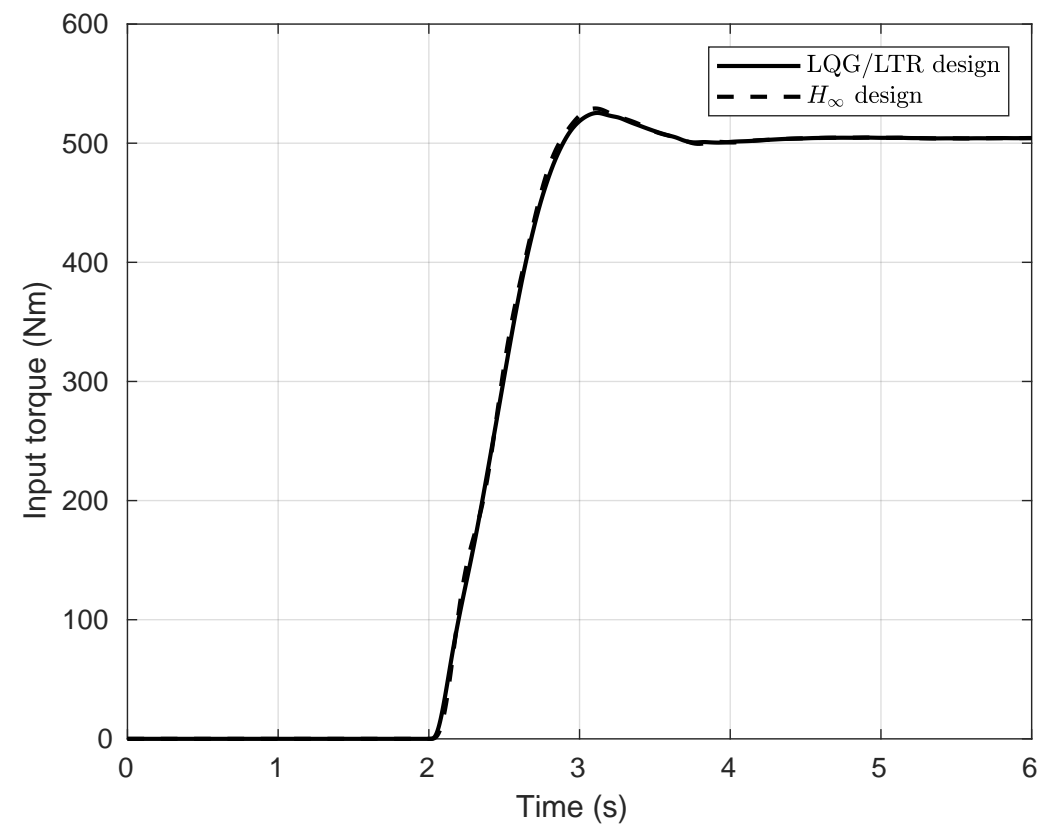

Source: Author.

\subsubsection{Sinusoidal steer test}

The sinusoidal steer test aims to evaluate the region of disturbance rejection, which was defined as one of the objectives in the control designs. In Section 7.1, it was defined that the control system will reduce disturbances in frequencies below $0.7 \mathrm{~Hz}$. In order to check this assumption, three simulations will be conducted with sinusoidal signals 
with different frequencies, $0.2 \mathrm{~Hz}, 0.7 \mathrm{~Hz}$ and $2 \mathrm{~Hz}$, where the vehicle is supposed to be at $40 \mathrm{~km} / \mathrm{h}$ and the driver slue the hand wheel for 30 degrees. This signal represents the handwheel angle introduced by the driver. The Figure 68 shows the lateral accelerations resulted by the three sinusoidal maneuvers, and they will be the disturbance inputs of the hybridized models.

Figure 68: Sinusoidal input to evaluate the disturbance rejection.

(a) Frequency: $0.2 \mathrm{~Hz}$

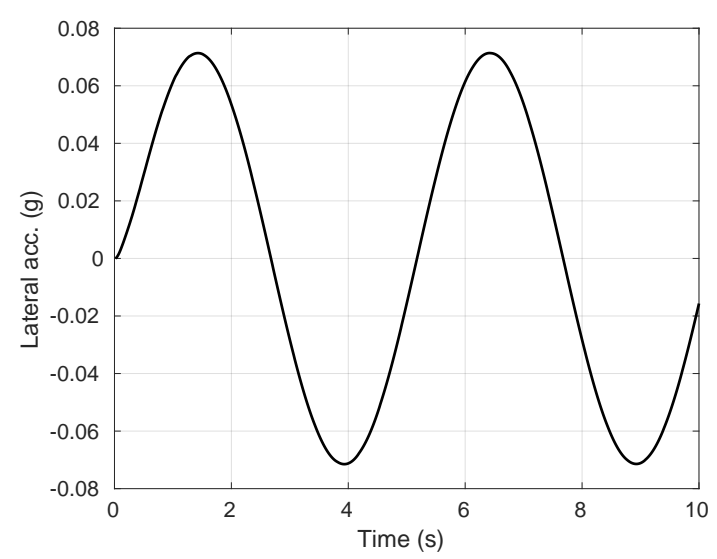

(b) Frequency: $0.7 \mathrm{~Hz}$

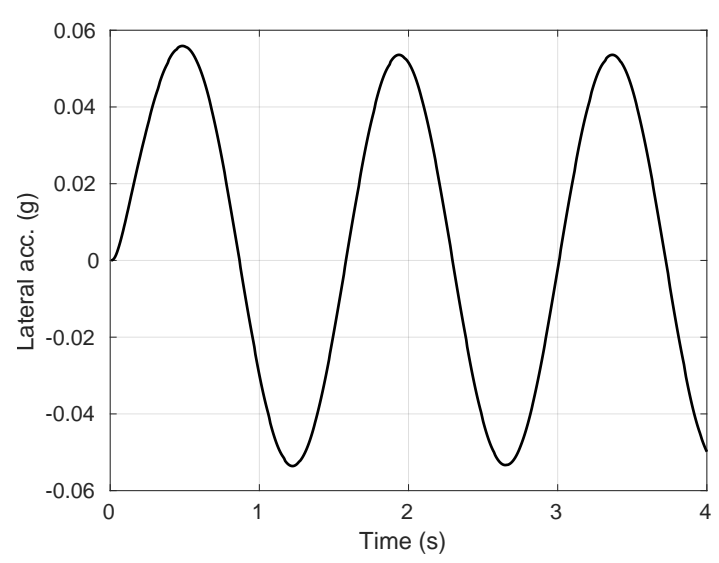

(c) Frequency: $2 \mathrm{~Hz}$

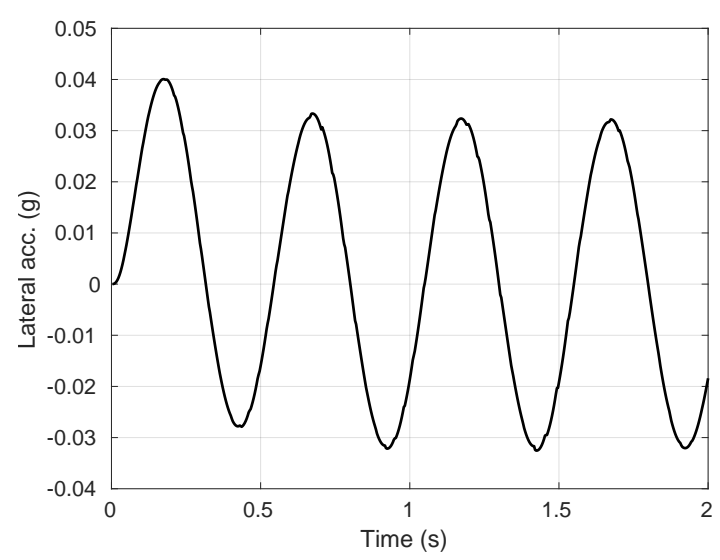

Source: Author.

The Figure 69 shows the roll angle for each of the maneuvers. The Figure 69a shows a roll angle reduction of $85 \%$ at steady-state peaks between the passive and the controlled system, where both controllers, LQG/LTR and $H_{\infty}$ loop-shaping has a very similar response. In the Figure 69b, the frequency of the disturbance input is exactly the same as the boundary frequency chosen as the requirement for the rejection of disturbance. In this case, the amplitude of the roll angle is reduced from 0.44 degree (passive anti-roll 
bar) to 0.2 degree (active anti-roll), meaning a reduction of $54 \%$. Again, the controllers have gotten the same behavior. The third case, shown in Figure 69c, takes into account an lateral acceleration at frequency of $2 \mathrm{~Hz}$. This frequency is out of the boundary specified by the project in the Section 7.1 and, as consequence, it is seen that anyone of the controllers is able to minimize the roll angle.

Figure 69: Roll angle response to the sinusoidal input at different frequencies.

(a) Frequency: $0.2 \mathrm{~Hz}$

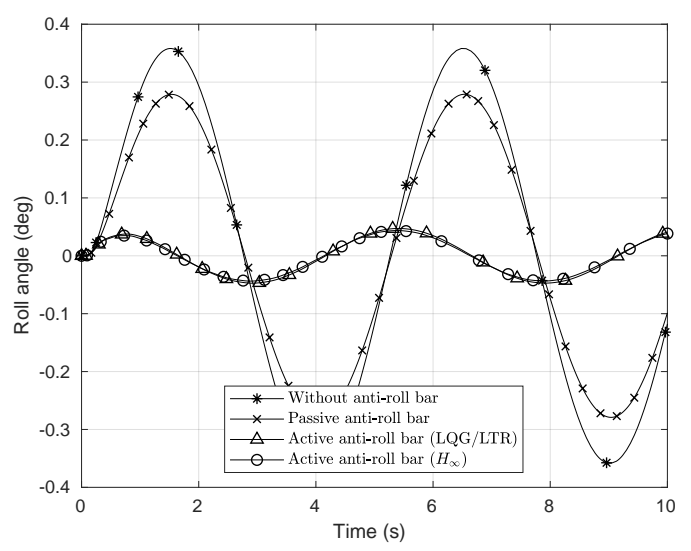

(b) Frequency: $0.7 \mathrm{~Hz}$

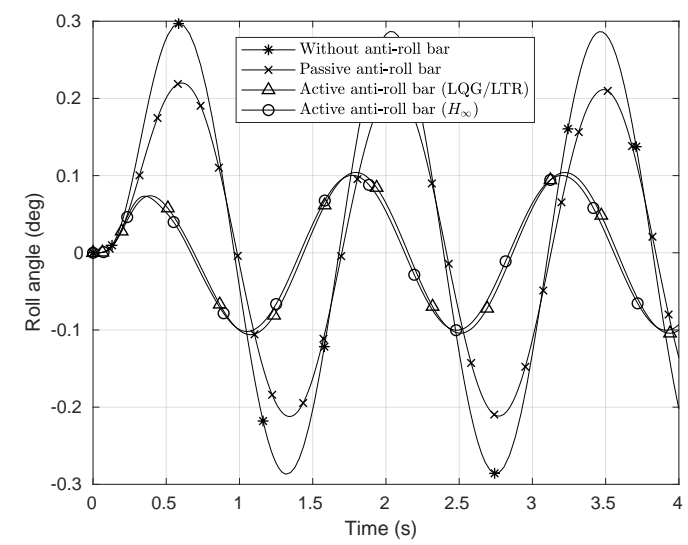

(c) Frequency: $2 \mathrm{~Hz}$

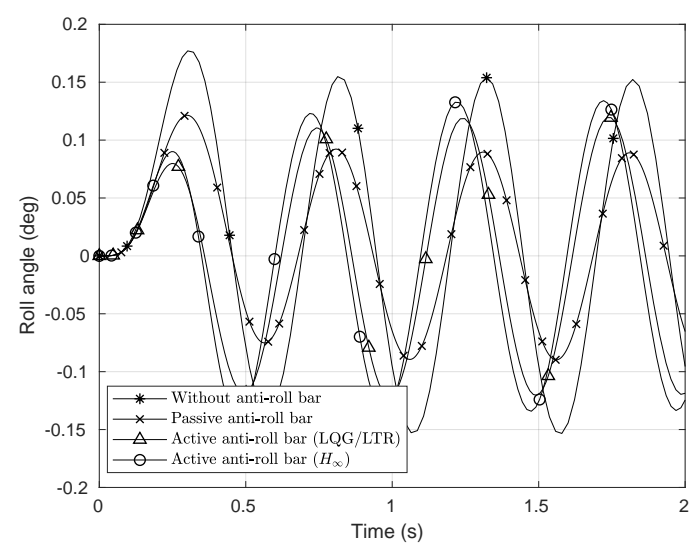

Source: Author.

The rate of the roll angle is shown in Figure 70. The analysis of the roll rate is equal to that performed to the roll angle: for disturbance frequencies above the limit specified for disturbance rejection, the controllers are not efficient. The Table 8, Table 9 and Table 10 show the comparison of RMS values among the systems for the three frequencies considered $(0.2 \mathrm{~Hz}, 0.7 \mathrm{~Hz}$ and $2 \mathrm{~Hz})$. As expected, the passive anti-roll bar unveil an improve in comparison with the system without anti-roll bar independently of 
the frequency. This happens because the system is passive and is not a feedback system, which naturally characterizes an open-loop control system. Then, the passive system will be always better than the system without the anti-roll bar. The same is not true for the active system. Table 10 shows that both controllers does not presented a relevant action on the roll angle. The LQG/LTR improved $7.7 \%$ where the $H_{\infty}$ controller worsened 7.7 , and this is not conclusive. However, both controllers worsened the rate of the roll angle above $20 \%$. Therefore, one can conclude that the specifications are respected by the controllers.

Figure 70: Roll rate for the sinusoidal input at different frequencies.

(a) Frequency: $0.2 \mathrm{~Hz}$

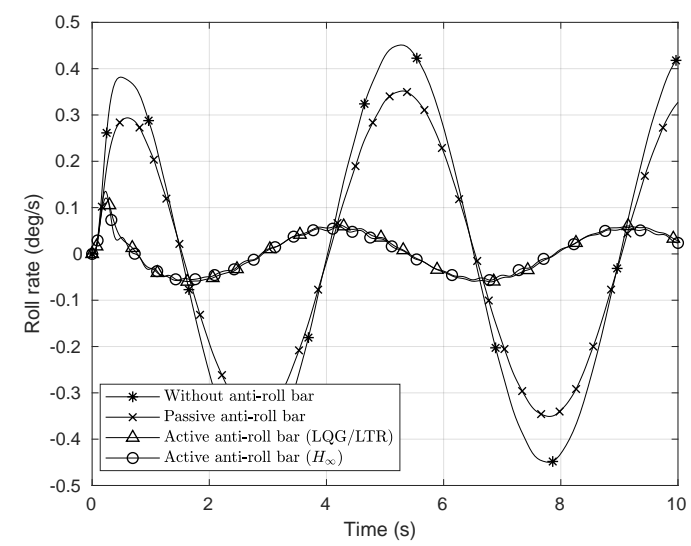

(b) Frequency: $0.7 \mathrm{~Hz}$

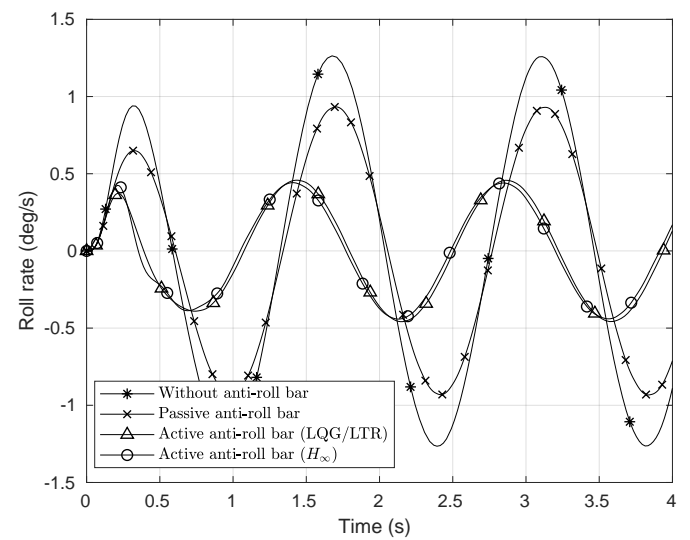

(c) Frequency: $2 \mathrm{~Hz}$

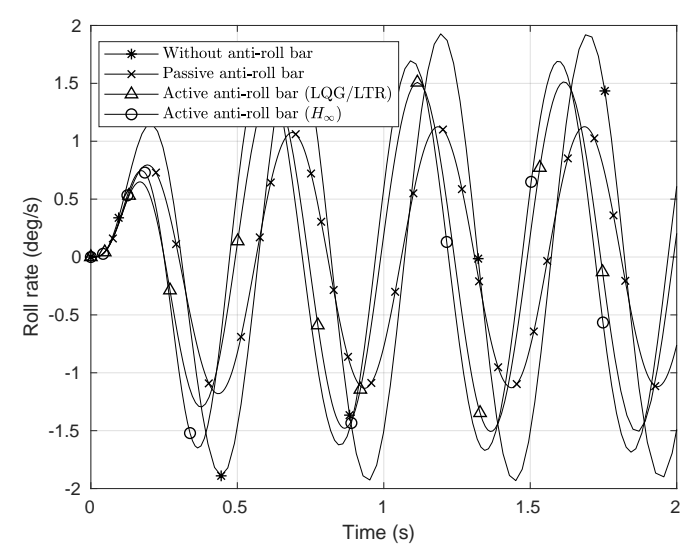

Source: Author. 
Table 8: Percentage of improvement in $40 \mathrm{~km} / \mathrm{h}$ sinusoidal steer test at $0.2 \mathrm{~Hz}$.

\begin{tabular}{|c|c|c|c|}
\hline \multicolumn{2}{|c|}{ Description } & Roll Angle (Degree) & Roll Rate (Degree/s) \\
\hline Without bar & RMS & 0.0101 & 0.0126 \\
\hline Passive bar & RMS & 0.0080 & 0.0099 \\
\hline \multirow{2}{*}{ LQG/LTR } & RMS & 0.0013 & 0.0018 \\
\cline { 2 - 4 } & Improvement (Without/passive) (\%) & $-87.1 /-83.7$ & $-85.7 /-81.8$ \\
\hline \multirow{2}{*}{$H_{\infty}$} & RMS & 0.0012 & 0.0017 \\
\cline { 2 - 4 } & Improvement (Without/passive) (\%) & $-88.1 /-85.0$ & $-86.5 /-82.8$ \\
\hline
\end{tabular}

Source: Author.

Table 9: Percentage of improvement in $40 \mathrm{~km} / \mathrm{h}$ sinusoidal steer test at $0.7 \mathrm{~Hz}$.

\begin{tabular}{|c|c|c|c|}
\hline \multicolumn{2}{|c|}{ Description } & Roll Angle (Degree) & Roll Rate (Degree/s) \\
\hline Without bar & RMS & 0.0076 & 0.0326 \\
\hline Passive bar & RMS & 0.0058 & 0.0239 \\
\hline \multirow{2}{*}{ LQG/LTR } & RMS & 0.0023 & 0.0123 \\
\cline { 2 - 4 } & Improvement (Without/passive) (\%) & $-69.7 /-60.3$ & $-62.3 /-48.5$ \\
\hline \multirow{2}{*}{$H_{\infty}$} & RMS & 0.0023 & 0.0119 \\
\cline { 2 - 4 } & Improvement (Without/passive) (\%) & $-69.7 /-60.3$ & $-63.5 /-50.2$ \\
\hline
\end{tabular}

Source: Author.

Table 10: Percentage of improvement in $40 \mathrm{~km} / \mathrm{h}$ sinusoidal steer test at $2 \mathrm{~Hz}$.

\begin{tabular}{|c|c|c|c|}
\hline \multicolumn{2}{|c|}{ Description } & Roll Angle (Degree) & Roll Rate (Degree/s) \\
\hline Without bar & RMS & 0.0018 & 0.0190 \\
\hline Passive bar & RMS & 0.0013 & 0.0124 \\
\hline \multirow{2}{*}{ LQG/LTR } & RMS & 0.0012 & 0.0 .0153 \\
\cline { 2 - 4 } & Improvement (Without/passive) (\%) & $-33.3 /-7.7$ & $-19.5 /+23.4$ \\
\hline \multirow{2}{*}{$H_{\infty}$} & RMS & 0.0014 & 0.0160 \\
\cline { 2 - 4 } & Improvement (Without/passive) (\%) & $-22.2 /+7.7$ & $-15.8 /+29.0$ \\
\hline
\end{tabular}

Source: Author.

\subsubsection{Lane-change maneuver}

Originally known as the "moose test" the lane change maneuver was transferred to the International Standard ISO 3888-2 after a revision by the Association of the German Automotive Industry (VDA). The ISO and VDA lane-change test is used to evaluate the handling performance of a vehicle and is an integral part of the vehicle design procedures and vehicle assessment (FLEPS-DEZASSE et al., 2018). The single lane-change has been carried out with vehicle at speed of $90 \mathrm{~km} / \mathrm{h}$ by using the steering input at the handwheel 
of 90 degrees, as shown in Figure 71a. Consequently, this steer input generates the lateral acceleration shown in Figure 71b,

Figure 71: Lane-change maneuver at $90 \mathrm{~km} / \mathrm{h}$ : (a) handwheel and (b) lateral acceleration.

(a)

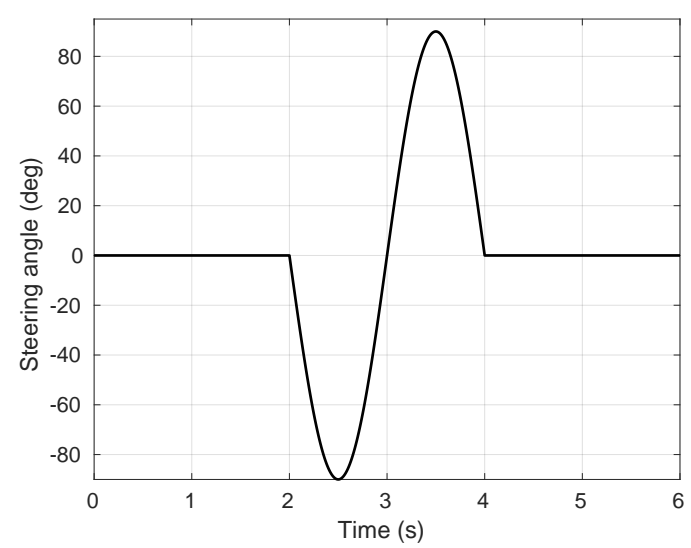

(b)

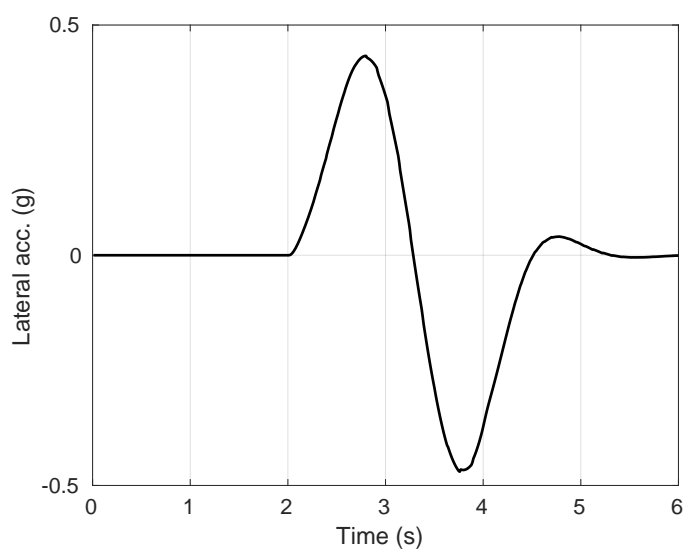

Source: Author.

Let's verify the behavior of the passive anti-roll bar. Figure 72 shows the vertical displacements of the left arm (see Figure 59) of the bar when subjected to the lanechange. As in the step steer test, it is observed the bending behavior through the nodes 1, 3 and 5. The node 1 is situated at the tip of the bar where is expected the larger displacement, about $0.8 \mathrm{~mm}$. The node 5 placed in the torsion region (central beam) has a smaller displacement, in this case $0.27 \mathrm{~mm}$.

Figure 72: Passive anti-roll bar: bending in y direction in $90 \mathrm{~km} / \mathrm{h}$ lane-change maneuver.

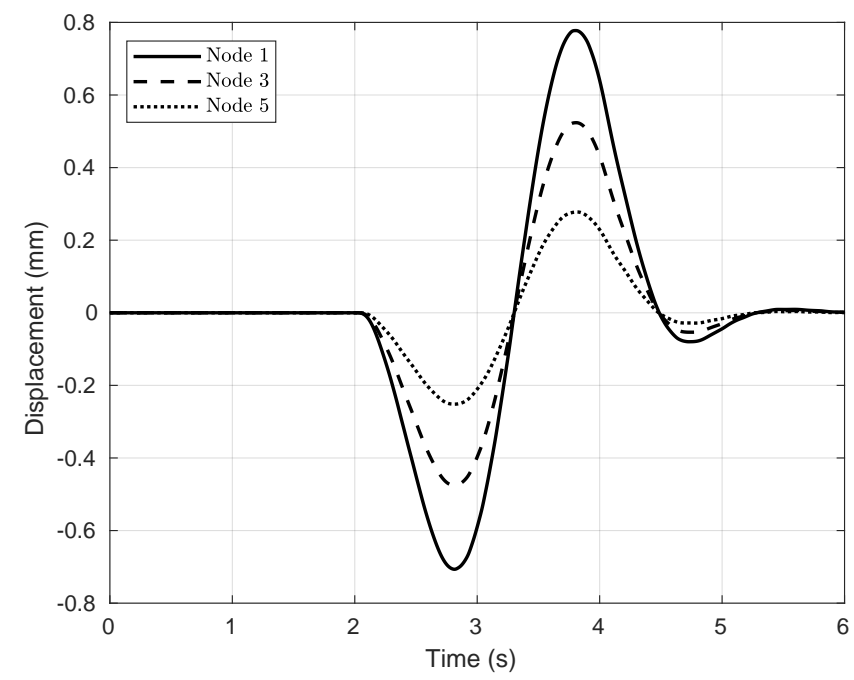

Source: Author. 
The Figure 73 shows the results of torsion for the nodes 5, 7, 9, 11 and 13 of the passive anti-roll bar. As a check of the model, the symmetry is verified where the node 9 experienced zero torsion whereas at the right and left tips of the central beam (nodes 5 and 13), the torsion $\theta$ has opposite direction with equal magnitudes.

Figure 73: Passive anti-roll bar: torsion in $90 \mathrm{~km} / \mathrm{h}$ lane-change maneuver.

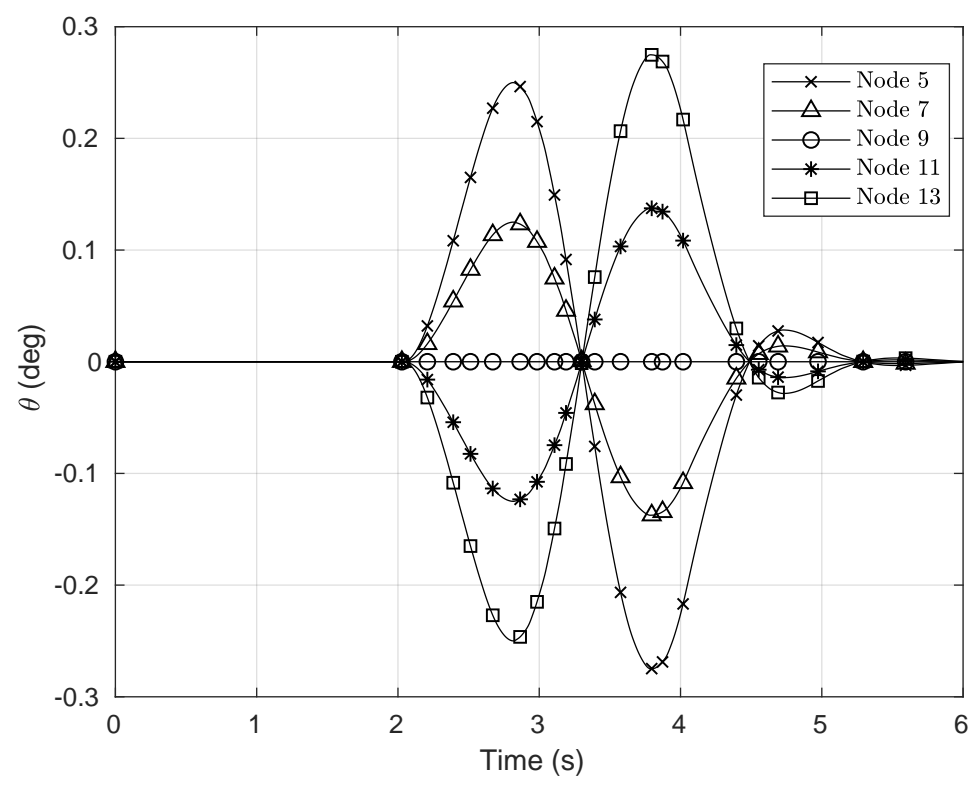

Source: Author.

Figure 74: Active anti-roll bar: bending in $y$ direction for the LQG/LTR and HLS in 90 $\mathrm{km} / \mathrm{h}$ lane-change maneuver.

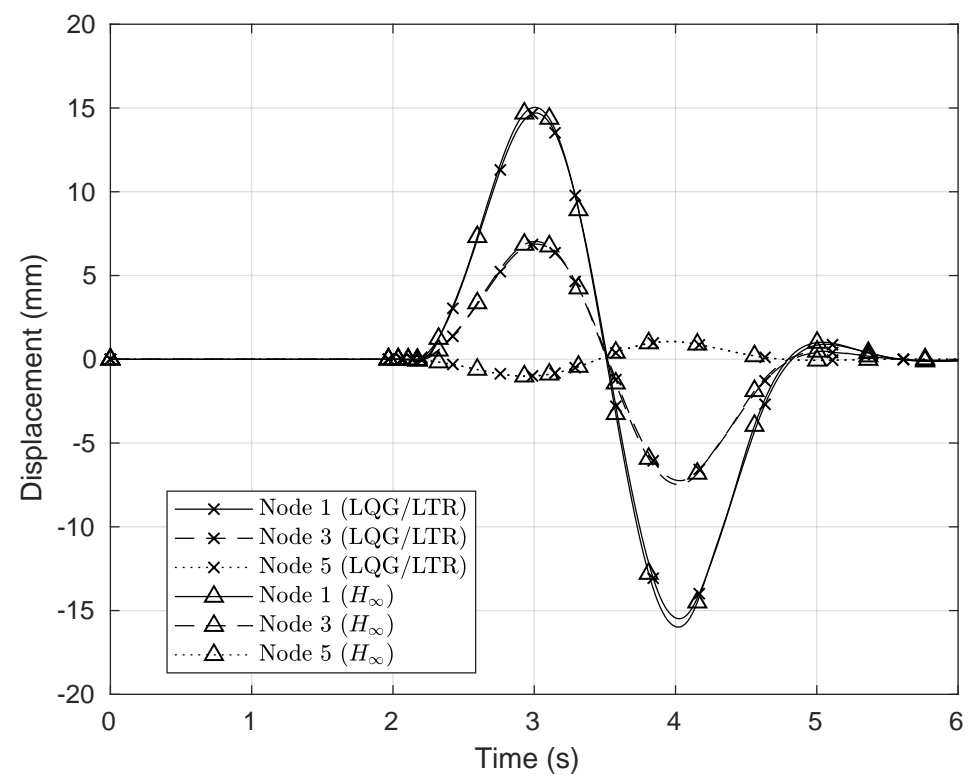

Source: Author. 
Looking now at the active anti-roll bar, the Figure 74 shows the bending behavior of the bar considering both controllers, LQG/LTR and $H_{\infty}$. Notice that the control actions are very similar, only with a slight difference in amplitude, which for the $H_{\infty}$ case is few higher. In the active case, to reduce the roll angle, the bar experience $15 \mathrm{~mm}$ at the tip due to control system.

The torsion results for both controllers are shown in Figure 75. The controller twists the bar to approximately 10 degrees at the connection with the source of torque, in this case represented by the electric motor. The LQG/LTR and $H_{\infty}$ controller leads the system to a similar response of torsion.

Figure 75: Torsion of the active anti-roll bar in $90 \mathrm{~km} / \mathrm{h}$ lane-change maneuver.

(a) LQG/LTR controller

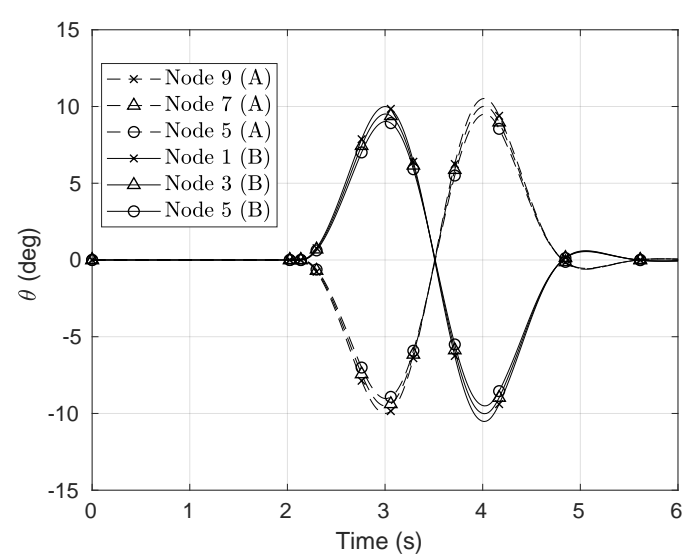

(b) $H_{\infty}$ controller

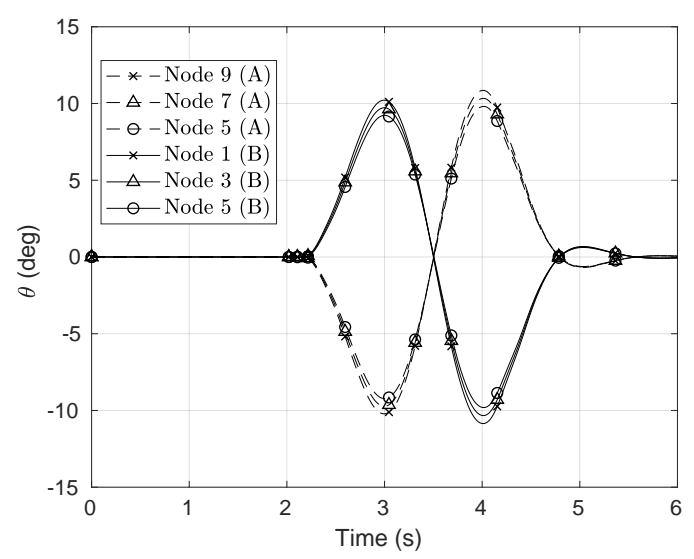

Source: Author.

The Figure 76 shows the roll angle, which is the controlled variable of the analysis. Without anti-roll bar, the roll angle reaches peak magnitude of 2.45 degree. Considering the passive bar, the peak roll angle reduces to 1.80 , that represents a decrease of $27 \%$. The model with active bar, leads the roll peak to 0.65 degree. The action of both controllers are the same, reducing the peak of the roll angle in $64 \%$, compared to the passive case.

The analysis of the rate of the roll angle is more relevant to comfort. The lower the variation of the roll angle, the better the comfort (RAJAMANI, 2012). Then, Figure 77 shows that the controlled system has a significant result on reduction of roll rate. 
Figure 76: Roll angle of the model without bar, with passive bar and active bar in 90 $\mathrm{km} / \mathrm{h}$ lane-change maneuver.

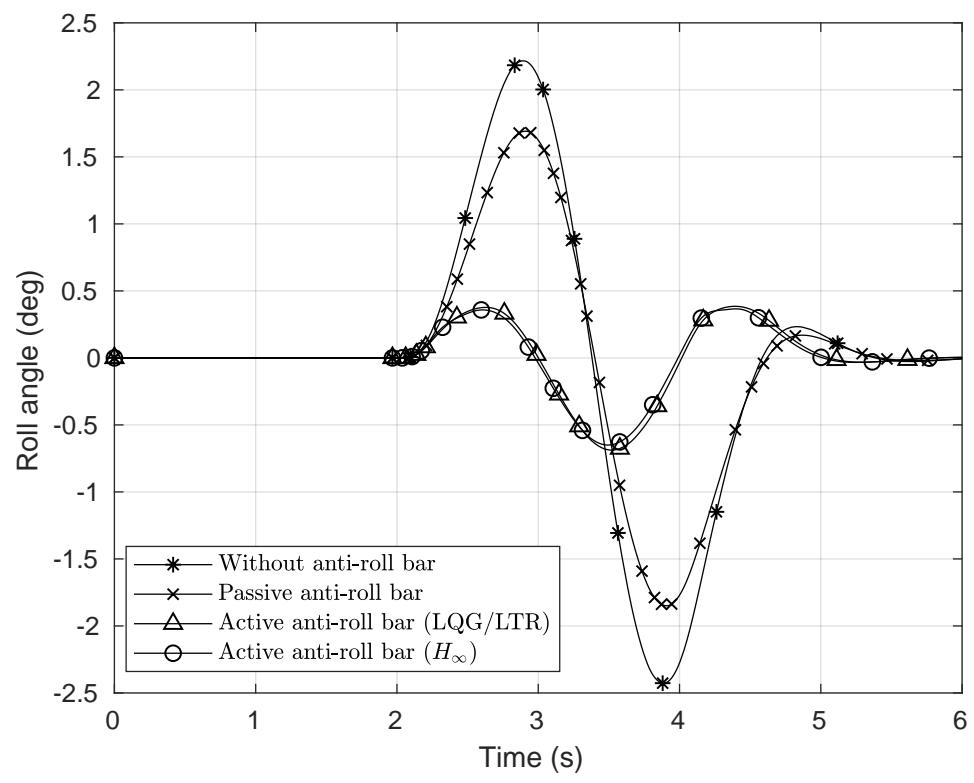

Source: Author.

Figure 77: Roll rate of the model without bar, with passive bar and active bar in $90 \mathrm{~km} / \mathrm{h}$ lane-change maneuver.

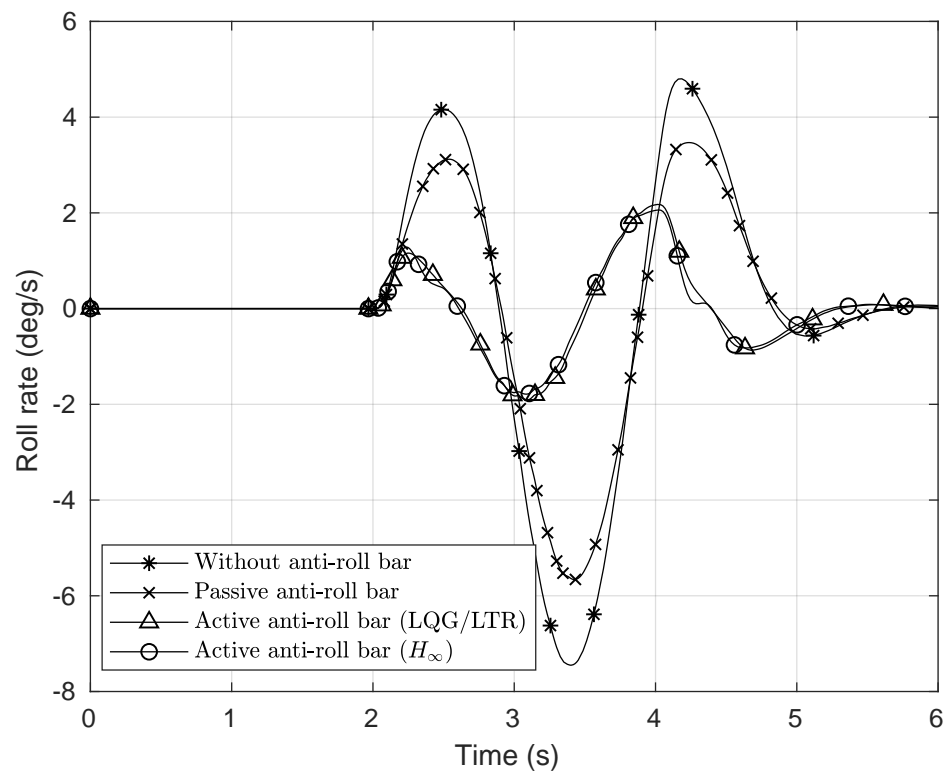

Source: Author.

Considering the RMS values of roll angle and rate of roll angle, Table 11 shows a comparison among the systems. The LQG/LTR design has reduced in $72.3 \%$ the roll angle compared to the passive system. On the other hand, in comparison with the roll 
rate, the same controller improved $61.1 \%$ The $H_{\infty}$ controller shows results very similar to the LQG/LTR: $73.4 \%$ for roll angle and $65.5 \%$ for roll rate. Therefore, there is no significant difference between the performance of the controllers and the choice for anyone should be done by implementation issues. It is important to notice the improvement of the passive anti-roll bar related to vehicle without anti-roll system. This improvement reaches $25 \%$ and is a cheap solution already used by most of the fleet.

Table 11: Percentage of improvement in $90 \mathrm{~km} / \mathrm{h}$ lane-change maneuver.

\begin{tabular}{|c|c|c|c|}
\hline \multicolumn{2}{|c|}{ Description } & Roll Angle (Degree) & Roll Rate (Degree/s) \\
\hline Without bar & RMS & 0.0239 & 0.0606 \\
\hline Passive bar & RMS & 0.0177 & 0.0478 \\
\hline \multirow{2}{*}{ LQG/LTR } & RMS & 0.0049 & 0.0186 \\
\cline { 2 - 4 } & Improvement (Without/passive) (\%) & $-79.5 /-72.3$ & $-69.3 /-61.1$ \\
\hline \multirow{2}{*}{$H_{\infty}$} & RMS & 0.0047 & 0.0165 \\
\cline { 2 - 4 } & Improvement (Without/passive) (\%) & $-80.3 /-73.4$ & $-72.8 /-65.5$ \\
\hline
\end{tabular}

Source: Author.

The control torque necessary in the application is shown in Figure 78 for both controllers. While the vehicle is taking other lane, the actuator, in both cases, provide a peak torque of $600 \mathrm{Nm}$ to mitigate the action of roll angle. As said in Section 7.1, it is assumed that all this torque is available in the actuator, not taking into account limitations on control action. However, looking to the specifications of an active stabilizer bar system from BWI Group, which is a premier chassis supplier that designs and manufactures brake and suspensions system for the global transportation market, the actuator allow torques from $900 \mathrm{Nm}$ up to $1500 \mathrm{Nm}$ (see https://www.bwigroup.com/product/asbs-active-stabilizerbar-systems). Therefore, the magnitude of the control torques provided by the simulations is compatible with the ones available in market, which confirms the representativeness of the models. 
Figure 78: Control signal for both controllers: torque provided by the electric motor in $90 \mathrm{~km} / \mathrm{h}$ lane-change maneuver.

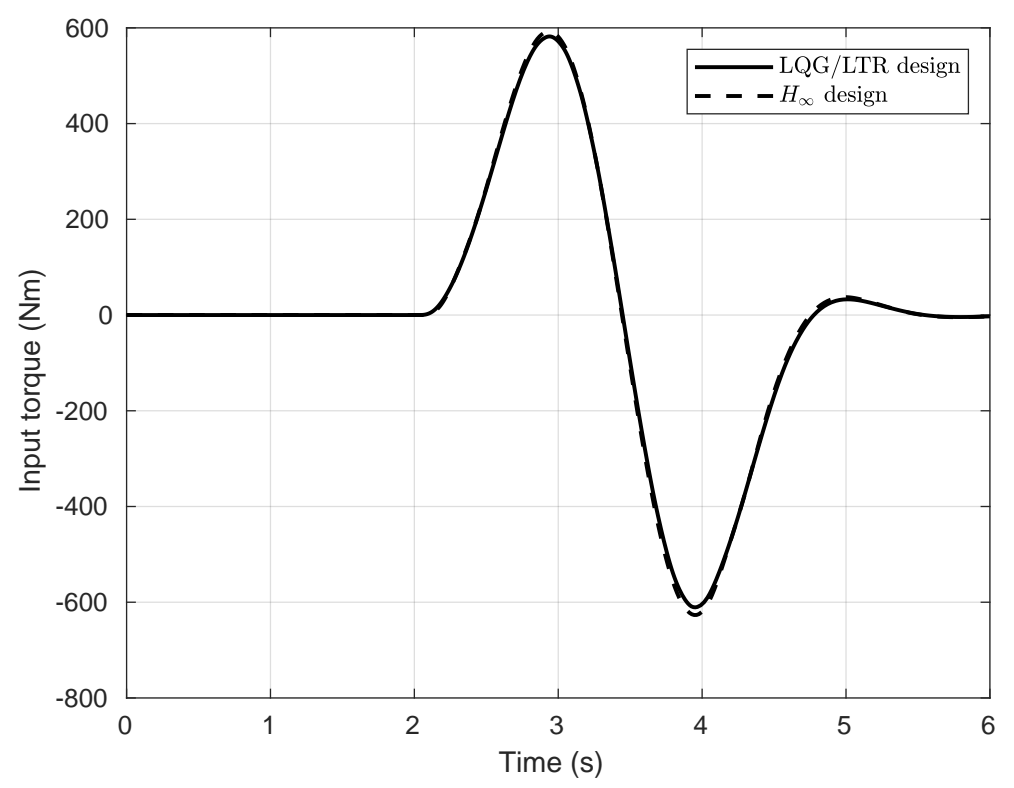

Source: Author.

Another important analysis to be done is the quantity of energy the motor consume when this maneuver is performed by a driver. In order to get this value, the Figure 79 show the angular velocity of the motor for both designed controllers.

Figure 79: Angular velocity of the electric motor for both controllers in $90 \mathrm{~km} / \mathrm{h}$ lanechange maneuver.

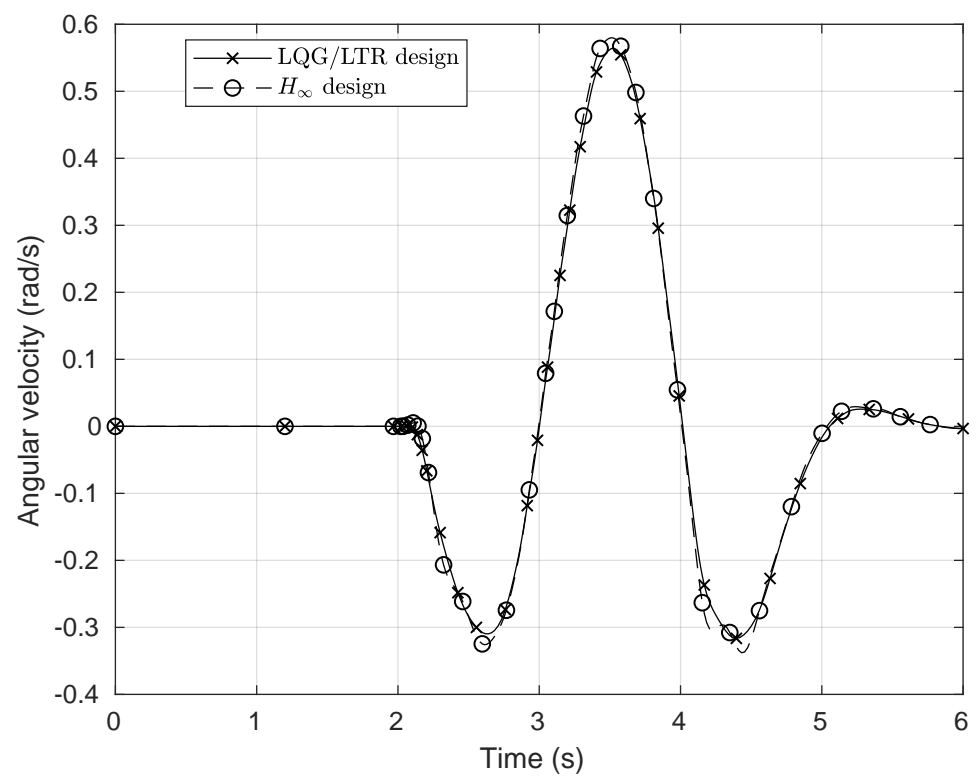

Source: Author. 
Physically, in the case of rotational motion, the calculation for power is the product of the torque by the rotational distance per unit of time, i.e. the angular velocity. Then, the instantaneous power equation is given as:

$$
P_{\text {rot }}(t)=T(t) \omega(t)
$$

where $T(t)$ is the instantaneous torque and $\omega(t)$ is the instantaneous angular velocity. The unit of the power $P_{\text {rot }}(t)$ is Watts. Using the Equation 7.8, the instantaneous power of the motor for both controllers in the lane-change maneuver is presented in Figure 80 . Figure 80: Instantaneous power of the electric motor for both controllers in $90 \mathrm{~km} / \mathrm{h}$ lane-change maneuver.

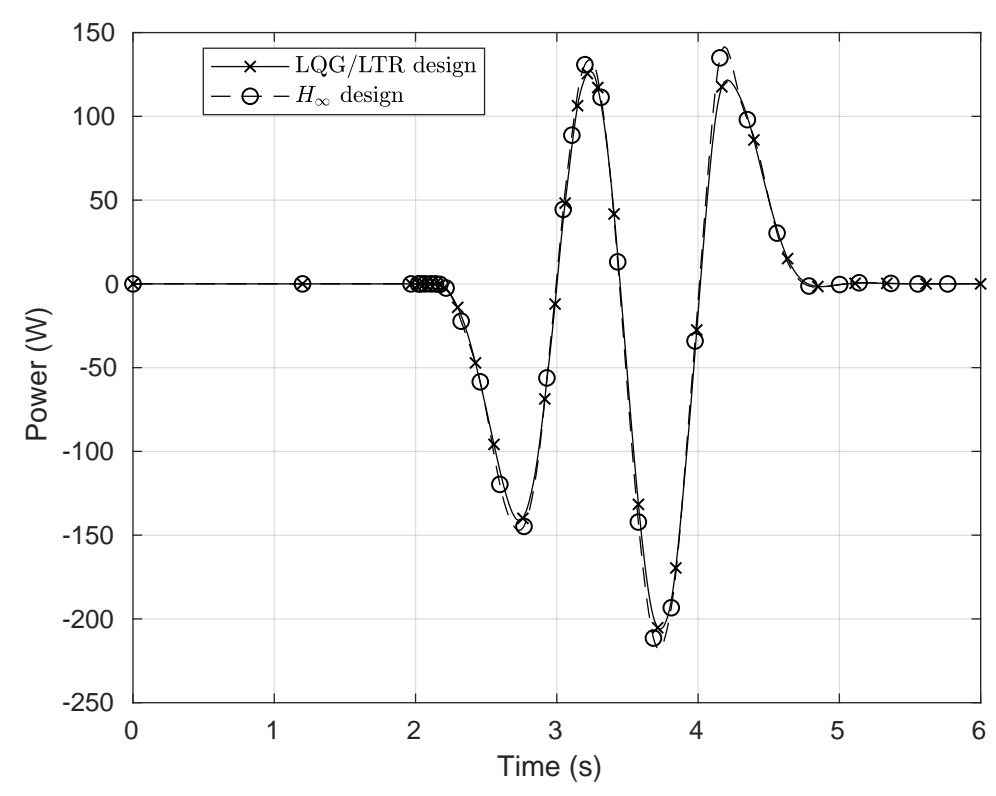

Source: Author.

The energy consumed by the motor, in Joules, can be calculated as follows:

$$
E_{u}=\int_{-\infty}^{\infty}\left|P_{r o t}(t)\right| d t
$$

Therefore, using the trapezoidal rule to integrate the instantaneous power in Figure 80, the energy consumed by the motor with the LQG/LTR control is 218.46 Joules and with the $H_{\infty}$ design is 229.53 Joules. The $H_{\infty}$ loop-shaping made the motor consume $5 \%$ more than the LQG/LTR control. 


\section{CONCLUSION}

In this work, it was presented how anti-roll bars can increase the driving safety and comfort by reducing the roll angle during cornering. Three different approaches were presented and compared: a vehicle without anti-roll system, one with passive anti-roll bar and another one with an active anti-roll bar, and this last one is supposedly controlled by an electric motor and an amplification module that provides the torque directly to anti-roll bar. The bars were modeled by FEM and the control designs LQG/LTR and $H_{\infty}$ loop-shaping were used to the active system.

As seen in the results, there is a significant opportunity to improve the safety and comfort of the current fleet by preventing vehicle to roll in cornering and get to rollover. Firstly, considering only the passive system, the roll behavior could be reduced about $25 \%$ compared to the system without anti-roll system. It is an expressive result considering the cost to implement a passive system. Currently, the passive anti-roll bar is already a safety item on cars. On the other hand, the active system shown even greater potential than the passive system. The results based on the lane change, presented an improvement up to $80 \%$ compared to the system without anti-roll bar related to the roll angle reduction. A more detailed analysis is necessary concerning the limitations of the control system as saturation of the torque, stresses and strain the bar is subjected, and so forth. Despite of this, the potential of improvement is evident.

It was designed two robust methods of control: the LQG/LTR method and the $H_{\infty}$ loop-shaping method. The results does not shown an evident better option for this application. Both controllers, using the same specifications, proposed a similar control action. Therefore, a choice between them considering performance is not possible in this case of study.

The control action performed by the motor show consistent values compared to commercial ones in this application regarding the magnitudes of torques reached in this work. Also, it was seen that the active system will add a consumption of approximately 220 Joules into the energy supplier system of the vehicle when it develops a lane-change ma- 
neuver. Therefore, the vehicle's battery system must be designed to be able to aggregate this application once it deals with an electric motor.

An structural analysis of the bars, looking at stresses and strain, the addition of the road inputs, neglected in this work, and a refinement of the control module could be considered as next steps. The results of the structural analysis could be updated for the geometry of the bar, making possible the realization in a real system as well as the other possibilities making the design more realistic. 


\section{REFERENCES}

NHTSA. Passenger vehicle occupant fatalities. 2016. Https://www.nhtsa.gov/. Accessed: 2018-02-30.

JAMIL, F. M.; ABDULLAH, M. Predictive control of vehicle roll dynamics with rear wheel steering. Proceedings of Innovative Research and Industrial Dialogue, American Control Conference, p. 135-136, 2010.

PHANOMCHOENG, G.; RAJAMANI, R. Prediction and prevention of tripped rollovers. Intelligent Transportation Systems Institute, Center for Transportation Studies, University of Minnesota, 2012.

CARLSON, C. R.; GERDES, J. C. Optimal rollover prevention with steer by wire and differential braking. Proceedings of the ASME International Mechanical Engineering Congress and Exposition, ASME, p. 345-355, November 2003.

CAMERON, J. T.; BRENNAN, S. A comparative, experimental study of model suitability to describe vehicle rollover dynamics for control design. International Mechanical Engineering Proceedings Congress and Exposition, ASME, November 2005.

CRAIG, E. B.; GERDES, J. C. Predictive control of vehicle roll dynamics with rear wheel steering. In: IEEE. American Control Conference. [S.1.], 2010. p. 1489-1494.

KIM, H.; PARK, Y. Investigation of robust roll motion control considering varying spedd and actuator dynamics. Mechatronics, Elsevier, v. 14, n. 1, p. 35-54, 2004.

YIM, S.; JEON, K.; YI, K. An investigation into vehicle rollover prevention by coordinated control of active anti-roll bar and electronic stability program. International Journal of Control, Automation and Systems, Springer, v. 10, n. 2, p. 275-287, 2012.

RAJAMANI, R. et al. Parameter and state estimation in vehicle roll dynamics. IEEE Transactions on Intelligent Transportation Systems, IEEE, v. 12, n. 4, p. 1558-1567, 2011.

AMMON, D. Modellbildung und Systementwicklung in der Fahrzeugdynamik. Vieweg+ Teubner Verlag, Stuttgart. [S.l.], 2013.

SCHRAMM MANFRED HILLER, R. B. a. D. Vehicle Dynamics: Modeling and Simulation. [S.l.]: Springer-Verlag Berlin Heidelberg, 2014.

RAJAMANI, R. Vehicle Dynamics and Control. 2. ed. [S.1.]: Springer US, 2012. (Mechanical Engineering Series).

GILLESPIE, T. D. Vehicle dynamics. Warren dale, 1997.

ÖTTGEN, O. Zur modellgestützten entwicklung eines mechatronischen fahrwerkregelungssystems für personenkraftwagen dissertation. Universität Duisburg-Essen, Düsseldorf: VDI-Verlag Google Scholar, 2005. 
MEIROVITCH, L. Dynamics and control of structures. [S.l.]: John Wiley \& Sons, 1990.

GAWRONSKI, W. Balanced control of flexible structures. [S.1.]: Springer, 2006. v. 211.

MOORE, B. Principal component analysis in linear systems: Controllability, observability, and model reduction. IEEE transactions on automatic control, IEEE, v. 26, n. 1, p. 17-32, 1981.

SKELTON, R. Cost decomposition of linear systems with applicatioin to model reduction. Int. Journal of Control, v. 32, p. 1031-1055, 1980.

BANG, H.; KWON, Y. W. The finite element method using MATLAB. [S.1.]: CRC press, 2000.

JUNKINS, J. L.; KIM, Y. Introduction to dynamics and control of flexible structures. [S.l.]: American Institute of Aeronautics and Astronautics, 1993.

TIMOSHENKO, S.; MACCULlOUGH, G. H. et al. Elements of strength of materials. Van Nostrand, 1949.

IRGENS, F. Continuum mechanics. [S.l.]: Springer Science \& Business Media, 2008.

COOK, R. D. et al. Concepts and applications of finite element analysis. [S.l.]: Wiley New York, 1974. v. 4.

BATHE, K.-J. Finite element procedures. [S.l.]: Klaus-Jurgen Bathe, 2006.

ABE, M. Vehicle handling dynamics: theory and application. [S.l.]: ButterworthHeinemann, 2015.

GU, D.-W.; PETKOV, P.; KOnSTANTINOV, M. M. Robust control design with MATLABß. [S.l.]: Springer Science \& Business Media, 2005.

SKOGESTAD, S.; POSTLETHWAITE, I. Multivariable feedback control: analysis and design. [S.l.]: Wiley New York, 2007. v. 2.

MACIEJOWSKI, J. M. Multivariable feedback design. electronic systems engineering series. Wokingham, England: Addison-Wesley, v. 6, p. 85-90, 1989.

CRUZ, J. d. Controle robusto multivariável. Universidade de São Paulo, São Paulo, Brasil, 1996.

ANDERSON, B.; MOORE, J.; MOLINARI, B. Linear optimal control. IEEE Transactions on Systems, Man, and Cybernetics, IEEE, n. 4, p. 559-559, 1972.

DOYLE, J. Guaranteed margins for lqg regulators. IEEE Transactions on Automatic Control, IEEE, v. 23, n. 4, p. 756-757, 1978.

MORRIS, J. M. The kalman filter: A robust estimator for some classes of linear quadratic problems. Information Theory, IEEE Transactions on, IEEE, v. 22, n. 5, p. 526-534, 1976.

PAPOULIS, A.; PILLAI, S. U. Probability, random variables, and stochastic processes. [S.l.]: Tata McGraw-Hill Education, 2002. 
ZAREI, J. et al. Design and comparison of lqg/ltr and $\mathrm{h}_{\infty}$ controllers for a vstol flight control system. Journal of the Franklin Institute, Elsevier, v. 344, n. 5, p. 577-594, 2007.

DOYLE, J. C.; STEIN, G. Robustness with observers. [S.1.], 1979.

MACFARLANE, D. Robust controller design using normalized coprime factor plant description. Lecture notes in control and information science, Springer-Verlag, v. 138, p. 51-131, 1990.

MCFARLANE, D.; GLOVER, K. A loop-shaping design procedure using h/sub infinity/synthesis. IEEE transactions on automatic control, IEEE, v. 37, n. 6, p. 759-769, 1992.

BOUKHNIFER, M.; FERREIRA, A. $H_{\infty}$ loop shaping bilateral controller for a two-fingered tele-micromanipulation system. IEEE Transactions on Control Systems Technology, IEEE, v. 15, n. 5, p. 891-905, 2007.

MUNIANDY, V. et al. Double anti-roll bar hardware-in-loop experiment for active anti-roll control system. Journal of Vibroengineering, v. 19, n. 4, 2017.

BHARANE, P. et al. Design, analysis and optimization of anti-roll bar. Journal of Engineering Research and Applications, v. 4, n. 9, p. 137-140, 2014.

FLEPS-DEZASSE, M. et al. Lpv feedforward control of semi-active suspensions for improved roll stability. Control Engineering Practice, Elsevier, v. 78, p. 1-11, 2018. 